\title{
Four-step access to the sesquiterpene natural product presilphiperfolan-1 $\beta$-ol and unnatural derivatives via supramolecular catalysis
}

Leonidas-Dimitrios Syntrivanis, ${ }^{\dagger}$ Ivana Némethová, ${ }^{\dagger}$ Dario Schmid,${ }^{\dagger}$ Shani Levi,,${ }^{\S}$ Alessandro Prescimone, ${ }^{\dagger}$ Fabian Bissegger, ${ }^{\dagger}$ Dan T. Major, ${ }^{\S}$ Konrad Tiefenbacher*,†, ${ }^{*}$

${ }^{\dagger}$ Department of Chemistry, University of Basel, Mattenstrasse 24a, 4058 Basel, Switzerland

*Department of Biosystems Science and Engineering, ETH Zurich, Mattenstrasse 24, 4058 Basel, Switzerland $\S$ Department of Chemistry, Bar-Ilan University, Ramat-Gan 52900, Israel

\section{Table of Contents}

1. General information

2. Synthesis of catalyst and cyclization substrates

General procedure A: Grignard addition using commercially available Grignard reagents

General procedure B: Grignard addition using self-made Grignard reagents

3. Cyclization studies

Screening scale reactions

Preparation and titration of $\mathrm{HCl}$ stock solution in chloroform

Preparation of water-satd. chloroform

Solvent screening results

GC traces of selected reaction conditions

Control experiments

Reactions of caryophyllene

4. Large scale reactions and isolation of products

General procedure C: Large scale cyclization reactions using resorcinarene capsule and $3 \mathrm{~mol} \%$ of $\mathrm{HCl}$

Note on the workup method used for large-scale reaction mixtures

5. Minor products of the cyclisation reaction

6. Comparison of spectral data for presilphiperfolan-1 $\beta-o l[(-)-4]$ with literature

7. Comparison of ${ }^{1} \mathrm{H}$ spectrum of alkene 10 with literature

8. References

Appendix A: Computational studies

Appendix B: Crystallographic details

Appendix C: NMR spectra of substrates

Appendix D: NMR spectra of products of cyclization reaction

Appendix E: DEPT and 2D-NMR spectra for ((2aS,2a $\left.{ }^{1} S, 4 a S, 7 S, 7 a S\right)-2 \mathrm{a}, 4 \mathrm{a}, 7-$ trimethyl2,2a,2a1,4a,5,6,7,7a-octahydro-1H-cyclopenta[cd]inden-4-yl)methanol (27) 
Appendix F: DEPT and 2D-NMR spectra for $\left(2 \mathrm{a} S, 2 \mathrm{a}^{1} R, 4 \mathrm{a} R, 7 S, 7 \mathrm{a} S\right)$-2a-ethyl-4,4,7trimethyldecahydro-7aH-cyclopenta[cd]inden-7a-ol [30]

Appendix G: DEPT and 2D-NMR spectra for $\left(2 \mathrm{a} S, 2 \mathrm{a}^{1} R, 4 \mathrm{a} R, 7 S, 7 \mathrm{a} S\right)-2 \mathrm{a}-$-butyl-4,4,7-

trimethyldecahydro-7aH-cyclopenta[cd]inden-7a-ol [32]

Appendix H: DEPT and 2D-NMR spectra for $\left(2 \mathrm{a} S, 2 \mathrm{a}^{1} R, 4 \mathrm{a} R, 7 S, 7 \mathrm{a} S\right)-2 \mathrm{a}-i s o-b u t y l-4,4,7-$

trimethyldecahydro-7aH-cyclopenta[cd]inden-7a-ol [34]

Appendix I: DEPT and 2D-NMR spectra for $\left(2 \mathrm{a} S, 2 \mathrm{a}^{1} R, 4 \mathrm{a} R, 7 S, 7 \mathrm{a} S\right)-2 \mathrm{a}-\mathrm{h} e x y 1-4,4,7-$

trimethyldecahydro-7aH-cyclopenta[cd]inden-7a-ol [36]

Appendix J: DEPT and 2D-NMR spectra for (2aS,2a $\left.{ }^{1} R, 4 a R, 7 S, 7 \mathrm{a} S\right)-2 \mathrm{a}-$ octyl-4,4,7-

trimethyldecahydro-7aH-cyclopenta[cd]inden-7a-ol [38] 


\section{General information}

Experimental: Reactions were carried out under an atmosphere of argon in dried glassware unless otherwise indicated. For the cyclisation reactions, no precaution against air and moisture was taken. Analytical thin-layer chromatography (TLC) was performed on Merck silica gel 60 F254 glass-backed plates, which were analysed after exposure to standard staining solutions (CAM: cerium ammonium molybdate, anisaldehyde or basic $\mathrm{KMnO}_{4}$ ). All NMR experiments were performed on a Bruker Avance Neo and a Bruker Avance III HD NMR spectrometer operating at $500 \mathrm{MHz}$ and $600 \mathrm{MHz}$ proton frequency, respectively. The instruments were equipped with a direct observe 5-mm BBFO smart probe $(500 \mathrm{MHz})$ or a five-channel cryogenic $5 \mathrm{~mm}$ QCI probe $(600 \mathrm{MHz})$. All probes were equipped with actively shielded z-gradients $(10 \mathrm{~A})$. The experiments were performed at $298 \mathrm{~K}$, VT NMR measurements were performed at $373 \mathrm{~K}$. Chemical shifts of ${ }^{1} \mathrm{H}$ NMR and ${ }^{13} \mathrm{C}$ NMR are given in ppm. The following solvent residual signals of the deuterated solvents were used as reference: $\mathrm{CDCl}_{3}: 7.26$ ppm $\left(\delta^{1} \mathrm{H}\right), 77.16 \mathrm{ppm}\left(\delta^{13} \mathrm{C}\right)$, benzene- $\mathrm{d}_{6}: 7.16 \mathrm{ppm}\left(\delta^{1} \mathrm{H}\right), 128.06 \mathrm{ppm}\left(\delta^{13} \mathrm{C}\right)$, toluene-d $\mathrm{d}_{8}: 7.09 \mathrm{ppm}$ $\left(\delta^{1} \mathrm{H}\right), 137.48 \mathrm{ppm}\left(\delta^{13} \mathrm{C}\right)$. Coupling constants $(J)$ are reported in Hertz $(\mathrm{Hz})$. Standard abbreviations indicating multiplicity were used as follows: $\mathrm{s}$ (singlet), $\mathrm{d}$ (doublet), $\mathrm{t}$ (triplet), dd (doublet of doublets), m (multiplet). Infrared spectra were recorded on a Perkin Elmer Spectrum Two FT-IR Spectrometer. GC analyses were carried out on a Shimadzu GC-2010 Plus instrument equipped with a FID detector and an Rtx-5 capillary column (length $=30 \mathrm{~m}$ ). Hydrogen was used as the carrier gas and the constantflow mode was used (flow rate $=40 \mathrm{~mL} / \mathrm{min}$ ) with a split ratio of 1:20. The following temperatureprogram was used: $60{ }^{\circ} \mathrm{C}$ for $3 \mathrm{~min}, 15^{\circ} \mathrm{C} / \mathrm{min}$ to $250{ }^{\circ} \mathrm{C}$, and $250{ }^{\circ} \mathrm{C}$ for $5 \mathrm{~min}$. For the determination of enantiopurity via GC, a Shimadzu GC-2010 Plus instrument equipped with a FID detector and an Rt-bDEXsm capillary column (length $=30 \mathrm{~m}$ ) was used. Hydrogen was used as the carrier gas and the constant-flow mode was used (flow rate $=50 \mathrm{~mL} / \mathrm{min}$ ) with a split ratio of 1:20. The following temperature-program was used: $60{ }^{\circ} \mathrm{C}$ for $1 \mathrm{~min}, 1{ }^{\circ} \mathrm{C} / \mathrm{min}$ to $220{ }^{\circ} \mathrm{C}$, and $220{ }^{\circ} \mathrm{C}$ for $10 \mathrm{~min}$. Highresolution mass spectra were obtained on a Thermo Scientific LTQ-FT Ultra via electrospray ionization (ESI) or a Finnigan MAT 8200 (EI) (ESI source parameters for positive polarity mode were: spray voltage, $4.0 \mathrm{kV}$; capillary temperature, $275^{\circ} \mathrm{C}$; capillary voltage, $48 \mathrm{~V}$; and tube lens, $-120 \mathrm{~V}$ ). Optical rotation was measured in an Anton Paar MCP 100 Circular Polarimeter operating on the sodium D-line $(589 \mathrm{~nm})$ and are reported as $[\alpha]_{\mathrm{D}}{ }^{\mathrm{T}}$ (concentration in $\mathrm{g} / 100 \mathrm{~mL}$, solvent). Ozonolysis was carried out using a BMT Ozone Generator $802 \mathrm{~N}$. $\mathrm{AgNO}_{3}$-coated silica was prepared according to the literature procedure. $^{1}$

Sources of chemicals: Deuterated chloroform $\left(\mathrm{CDCl}_{3}, 99.8 \%\right)$ was purchased from Cambridge Isotope Laboratories (stabilized over silver foil). Acetic acid, aluminum oxide (activated, basic, Brockmann I), benzene- $\mathrm{d}_{6},(-)$-caryophyllene oxide, $\beta$-caryophyllene, copper (II) acetate hydrate, ethanol $(99.9 \%)$, ethylmagnesium bromide ( $3.0 \mathrm{M}$ sol. in diethyl ether), hexylmagnesium bromide ( $0.8 \mathrm{M}$ sol. In THF), iso-butylmagnesium bromide $(2.0 \mathrm{M}$ sol. in diethyl ether), $n$-hexane (HPLC grade), methanol, methylmagnesium bromide (3.0 M sol. in diethyl ether), pentylmagnesium bromide (2.0 $\mathrm{M}$ sol. in diethyl ether), phenol red, semicarbazide hydrochloride, tetrabutylammonium bromide, trimethylamine, toluene- $\mathrm{d}_{8}$ and Zinc dust were purchased from Sigma-Aldrich. Anhydrous diethyl ether $\left(\mathrm{Et}_{2} \mathrm{O}\right)$ and anhydrous DCM were purchased from Acros Organics. Silica gel $(0.040-0.063 \mathrm{~mm}, 230-400$ mesh ASTM) was purchased from Merck KGaA. $n$-Decane was purchased from Fluka. All chemicals were used as received. Transfer of liquids with a volume ranging from 1 to $10 \mu \mathrm{L}$ or from 10 to $100 \mu \mathrm{L}$ was performed with a microman M1 pipette (Gilson, systematic error: $1.40 \%-1.60 \%$ ) equipped with $10 \mu \mathrm{L}$ or $100 \mu \mathrm{L}$ pipette tips, respectively. 


\section{Synthesis of catalyst and cyclization substrates}

Synthesis of resorcinarene 1: Synthesis of resorcinarene 1 was carried out as previously described. ${ }^{2}$

\section{Synthesis of cyclization substrates:}

$(1 R, 4 R, 6 R, 10 S)-4,12,12$-trimethyl-5-oxatricyclo[8.2.0.04,6] dodecan-9-one (kobusone, 21) ${ }^{3}$<smiles>C[C@@H]1CC[C@H]2C(C)(C)C[C@H]1C(=O)CCC[C@]2(C)O</smiles>

(-)-Caryophyllene oxide 20 (6.00 g, $27.2 \mathrm{mmol})$ was dissolved in DCM (150 mL) in a two-neck flask. The solution was cooled to $-78{ }^{\circ} \mathrm{C}$ and ozone was bubbled through until a blue color persisted; the mixture was then purged with oxygen until disappearance of the blue color. The reaction mixture was then placed under an atmosphere of Ar and $\mathrm{Zn}$ dust (2.67 g, $40.8 \mathrm{mmol})$ was added in one portion followed by $50 \%$ aq. acetic acid $(75 \mathrm{~mL})$. The mixture was stirred at $\mathrm{rt}$ for $18 \mathrm{~h}$. Water $(150 \mathrm{~mL})$ was then added, the layers were separated, and the aqueous layer extracted with DCM $(2 \times 70 \mathrm{~mL})$. The combined organic layers were washed with satd. aq. $\mathrm{NaHCO}_{3}$ solution $(4 \times 70 \mathrm{~mL})$ then brine $(50 \mathrm{~mL})$, dried over $\mathrm{MgSO}_{4}$, and the solvent removed in vacuo. The crude product 21 (colorless oil that solidifies in freezer, $5.92 \mathrm{~g}, 98 \%$ ) was used in the following steps with no further purification. Spectral data was consistent with literature. ${ }^{3}$

$(1 S, 9 R, E)-6,10,10$-trimethylbicyclo[7.2.0] undec-5-en-2-one $(22)^{3}$<smiles>CC1=CCC[C@@H]2[C@@H](CC2(C)C)C(=O)CC1</smiles>

Zn dust (15.9 g, $244.0 \mathrm{mmol})$ was suspended in EtOH $(62 \mathrm{~mL})$, and acetic acid (1.6 mL, $28.0 \mathrm{mmol})$ was added followed by $\mathrm{Cu}(\mathrm{OAc})_{2} \cdot \mathrm{H}_{2} \mathrm{O}(1.62 \mathrm{~g}, 8.1 \mathrm{mmol})$. The mixture was heated to $45^{\circ} \mathrm{C}$ for $45 \mathrm{~min}$, then cooled to $\mathrm{rt}$ and kobusone $21(2.00 \mathrm{~g}, 9.0 \mathrm{mmol})$ added as a solution in EtOH (5 mL, rinsing with $2 \times 5 \mathrm{~mL}$ ). The mixture was heated to reflux for $18 \mathrm{~h}$, then cooled to rt and filtered through celite. The solvent was removed in vacuo and the crude product purified via flash chromatography (cyclohexane/ethyl acetate, 12:1) to give the title compound 22 as a colorless oil $(1.49 \mathrm{~g}, 80 \%) .{ }^{13} \mathrm{C}$ spectral data was consistent with literature ${ }^{3}$ part of the reported ${ }^{1} \mathrm{H}$ spectral data was misassigned, the correct peak listing follows:

${ }^{1} \mathbf{H}$ NMR $\left(500 \mathrm{MHz}, \mathrm{CDCl}_{3}\right) \delta 5.25(\mathrm{dd}, J=8.1,7.1 \mathrm{~Hz}, 1 \mathrm{H}), 2.84(\mathrm{dt}, J=9.3,8.1 \mathrm{~Hz}, 1 \mathrm{H}), 2.77-2.66$ $(\mathrm{m}, 1 \mathrm{H}), 2.45-2.37(\mathrm{~m}, 1 \mathrm{H}), 2.25-2.10(\mathrm{~m}, 3 \mathrm{H}), 2.04(\mathrm{dd}, J=9.7,9.6 \mathrm{~Hz}, 1 \mathrm{H}), 1.92(\mathrm{td}, J=12.4,4.6$ $\mathrm{Hz}, 1 \mathrm{H}), 1.88-1.83(\mathrm{~m}, 1 \mathrm{H}), 1.76(\mathrm{~d}, J=1.4 \mathrm{~Hz}, 3 \mathrm{H}), 1.65-1.49(\mathrm{~m}, 3 \mathrm{H}), 1.02(\mathrm{~s}, 3 \mathrm{H}), 0.99(\mathrm{~s}, 3 \mathrm{H})$. 


\section{General procedure A: Grignard addition using commercially available Grignard reagents}

Ketone 22 (1.0 equiv.) was dissolved in anhydrous diethyl ether $(0.06 \mathrm{M})$ under an Ar atmosphere. The reaction mixture was cooled to $0{ }^{\circ} \mathrm{C}$. At this temperature, the Grignard reagent (1.5 equiv.) was added dropwise. The mixture was stirred at $\mathrm{rt}$ until complete conversion, then brine $(20 \mathrm{~mL})$ was added. The layers were separated and the aqueous layer was extracted with $\mathrm{Et}_{2} \mathrm{O}(2 \times 20 \mathrm{~mL})$. The combined organic layers were dried over $\mathrm{MgSO}_{4}$ and the solvent removed in vacuo; the crude product was then purified via flash chromatography.

\section{General procedure B: Grignard addition using self-made Grignard reagents}

A two-neck round bottom flask equipped with condenser was filled with Mg-turnings (3.5 equiv.) and a crystal of iodine under Ar atmosphere. Anhydrous THF or anhydrous $\mathrm{Et}_{2} \mathrm{O}$ was added $(0.5 \mathrm{M})$ followed by the slow addition of alkyl bromide (3.5 equiv.). The reaction vessel was gently heated with a heat gun in order to initiate the reaction. Upon the full consumption of the Mg turnings, the mixture was used in the following reaction.

Ketone 22 (1.0 equiv.) was dissolved in anhydrous $\mathrm{Et}_{2} \mathrm{O}$ or anhydrous $\mathrm{THF}(0.06 \mathrm{M})$ under $\mathrm{Ar}$ atmosphere. The reaction mixture was cooled to $0{ }^{\circ} \mathrm{C}$. At this temperature, the Grignard reagent (2.0 equiv.) was added dropwise. The mixture was stirred at $\mathrm{rt}$ until complete conversion, then brine (20 $\mathrm{mL})$ was added. The layers were separated and the aqueous layer was extracted with $\mathrm{Et}_{2} \mathrm{O}(2 \times 20 \mathrm{~mL})$. The combined organic layers were dried over $\mathrm{MgSO}_{4}$ and the solvent removed in vacuo; the crude product was then purified via flash chromatography.

$(1 S, 2 R, 9 R, E)-2,6,10,10-$ tetramethylbicyclo[7.2.0]undec-5-en-2-ol $(23)^{3}$

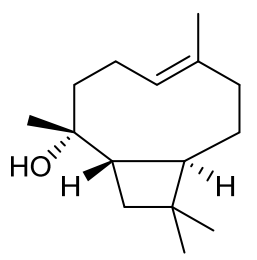

Compound 23 was synthesised according to the General Procedure A using ketone 22 (2.24 mmol, 500 $\mathrm{mg}$ ) and $3.0 \mathrm{M}$ solution of methylmagnesium bromide in $\mathrm{Et}_{2} \mathrm{O}$ for $2 \mathrm{~h}$. Flash chromatography was performed using pentane/ $\mathrm{Et}_{2} \mathrm{O}(12: 1)$ as mobile phase. It was isolated as colourless oil.

Yield: $520 \mathrm{mg}, 97 \%$.

NMR spectra of this compound exhibit broad peaks due to slow interconversion of conformers. The VT- ${ }^{1} \mathrm{H}$ NMR spectrum measured at $100{ }^{\circ} \mathrm{C}$ was consistent with that reported in the literature. ${ }^{3}$

$(1 S, 2 R, 9 R, E)-2$-ethyl-6,10,10-trimethylbicyclo[7.2.0] undec-5-en-2-ol (29)<smiles>CC[C@H]1CC/C=C(\C)CC[C@H]2[C@@H]1CC2(C)C</smiles>

Compound 29 was synthesised according to the General Procedure A using ketone $22(0.727 \mathrm{mmol}$, $150 \mathrm{mg}$ ) and 3.0 $\mathrm{M}$ solution of ethylmagnesium bromide in $\mathrm{Et}_{2} \mathrm{O}$ for $2 \mathrm{~h}$. Flash chromatography was 
performed using pentane/ $\mathrm{Et}_{2} \mathrm{O}(12: 1)$ as mobile phase. It was isolated as a slightly yellow oil. NMR spectra of this compound exhibit broad peaks at room temperature due to slow interconversion of conformers. The VT- ${ }^{1} \mathrm{H}$ NMR and ${ }^{13} \mathrm{C}$ NMR spectra were measured at $100{ }^{\circ} \mathrm{C}$.

Yield: $163 \mathrm{mg}, 95 \%$

$\mathbf{R}_{f} 0.34$ (pentane / $\mathrm{Et}_{2} \mathrm{O}, 9: 1$ )

IR $v_{\max }\left(\right.$ thin film) $/ \mathrm{cm}^{-1} 3492 \mathrm{br}, 2921 \mathrm{~s}, 2854 \mathrm{~m}, 1460 \mathrm{~m}, 1449 \mathrm{~m}, 1381 \mathrm{~m}, 1366 \mathrm{~m}, 1277 \mathrm{~m}, 1033 \mathrm{~m}, 942 \mathrm{~m}$, $875 \mathrm{~m}$

${ }^{1}$ H NMR $(500 \mathrm{MHz}$, Toluene-d, $373 \mathrm{~K}) \delta 5.45(\mathrm{t}, J=8.0 \mathrm{~Hz}, 1 \mathrm{H}), 2.07(\mathrm{~d}, J=13.5 \mathrm{~Hz}, 1 \mathrm{H}), 1.85(\mathrm{dt}$, $J=11.6,5.8 \mathrm{~Hz}, 1 \mathrm{H}), 1.82-1.77(\mathrm{~m}, 1 \mathrm{H}), 1.73(\mathrm{t}, J=9.3 \mathrm{~Hz}, 1 \mathrm{H}), 1.70-1.59(\mathrm{~m}, 2 \mathrm{H}), 1.58(\mathrm{~s}, 3 \mathrm{H})$, $1.56-1.37(\mathrm{~m}, 5 \mathrm{H}), 1.37-1.27(\mathrm{~m}, 1 \mathrm{H}), 1.24-1.12(\mathrm{~m}, 1 \mathrm{H}), 0.95(\mathrm{~s}, 3 \mathrm{H}), 0.92(\mathrm{~s}, 3 \mathrm{H}), 0.86(\mathrm{~s}, 1 \mathrm{H})$, $0.74(\mathrm{t}, \mathrm{J}=7.5 \mathrm{~Hz}, 3 \mathrm{H})$.

${ }^{13}$ C NMR $\left(126 \mathrm{MHz}\right.$, Toluene-d $\left.\mathrm{d}_{8}, 373 \mathrm{~K}\right) \delta$ 134.7, 74.8, 51.6, 47.8, 40.0, 39.3, 37.6, 36.6, 32.3, 31.2, $30.1,23.6,23.5,17.1,8.6$.

NOTE: In the ${ }^{1} \mathrm{H}$ NMR, 1 proton resonance $(2.13 \mathrm{ppm})$ is hidden under the solvent residual signal of $\mathrm{CH}_{3}(2.12 \mathrm{ppm})$ of toluene- $\mathrm{d}_{8}$, as can be seen in the HSQC spectrum. Furthermore, in the ${ }^{13} \mathrm{C} \mathrm{NMR}$, the disubstituted alkene resonance (125.1) is also hidden under the solvent residual signal of toluene- $\mathrm{d}_{8}(\mathrm{t}$, $125.5 \mathrm{ppm})$.

HRMS $\left(\mathrm{ESI}^{+}\right) \mathrm{m} / \mathrm{z}$ calculated for $\mathrm{C}_{16} \mathrm{H}_{28} \mathrm{NaO}\left[\mathrm{M}+\mathrm{Na}^{+}\right]: 259.2032$; found 259.2028

\section{$(1 S, 2 R, 9 R, E)-2-b u t y l-6,10,10-t r i m e t h y l b i c y c l o[7.2 .0]$ undec-5-en-2-ol (31)}<smiles>CC1=CCC[C@H](Br)[C@H]2CC(C)(C)[C@H]2CC1</smiles>

Compound 31 was synthesised according to the General Procedure B using ketone $22(0.485 \mathrm{mmol}$, $100 \mathrm{mg}$ ) and $0.5 \mathrm{M}$ butylmagnesium bromide in $\mathrm{Et}_{2} \mathrm{O}$ for $2 \mathrm{~h}$. Flash chromatography was performed using pentane/ $\mathrm{Et}_{2} \mathrm{O}(12: 1)$ as mobile phase. It was isolated as a colourless oil. NMR spectra of this compound exhibit broad peaks at room temperature due to slow interconversion of conformers. The VT- ${ }^{1} \mathrm{H}$ NMR and ${ }^{13} \mathrm{C}$ NMR spectra were measured at $100{ }^{\circ} \mathrm{C}$.

Yield: $109 \mathrm{mg}, 85 \%$

$\mathbf{R}_{\boldsymbol{f}} 0.42$ (pentane / $\left.\mathrm{Et}_{2} \mathrm{O}, 9: 1\right)$

IR $v_{\max }\left(\right.$ thin film) $/ \mathrm{cm}^{-1} 3495 \mathrm{br}, 2924 \mathrm{~s}, 2858 \mathrm{~s}, 1448 \mathrm{~m}, 1379 \mathrm{~m}, 1366 \mathrm{~m}, 1038 \mathrm{~m}, 989 \mathrm{~m}, 877 \mathrm{~m}$

${ }^{1}$ H NMR $(500 \mathrm{MHz}$, Toluene-d $8,373 \mathrm{~K}) 5.46(\mathrm{t}, J=8.0 \mathrm{~Hz}, 1 \mathrm{H}), 2.07$ (d, $\left.J=12.5 \mathrm{~Hz}, 1 \mathrm{H}\right), 1.90-1.78$ $(\mathrm{m}, 2 \mathrm{H}), 1.77-1.63(\mathrm{~m}, 3 \mathrm{H}), 1.59(\mathrm{~s}, 3 \mathrm{H}), 1.57-1.38(\mathrm{~m}, 4 \mathrm{H}), 1.36-1.31(\mathrm{~m}, 1 \mathrm{H}), 1.30-1.17(\mathrm{~m}$, $4 \mathrm{H}), 1.17-1.10(\mathrm{~m}, 2 \mathrm{H}), 0.96(\mathrm{~s}, 3 \mathrm{H}), 0.92(\mathrm{~s}, 3 \mathrm{H}), 0.89(\mathrm{t}, J=7.1 \mathrm{~Hz}, 3 \mathrm{H})$.

${ }^{13}$ C NMR (126 MHz, Toluene-d 8 , 373K) $\delta$ 134.7, 74.7, 51.9, 47.8, 44.3, 40.1, 40.0, 37.7, 32.3, 31.2, $30.1,27.0,24.1,23.7,23.5,17.2,14.3$.

NOTE: In the ${ }^{1} \mathrm{H}$ NMR, 1 proton resonance (2.14 ppm) is overlapping with the solvent residual signal of $\mathrm{CH}_{3}(2.12 \mathrm{ppm})$ of toluene- $\mathrm{d}_{8}$, as can be seen in the HSQC spectrum. Furthermore, in the ${ }^{13} \mathrm{C}$ NMR, 
the disubstituted alkene resonance (125.2) is hidden under the solvent residual signal of toluene- $\mathrm{d}_{8}(\mathrm{t}$, $125.5 \mathrm{ppm})$.

HRMS $\left(\mathrm{ESI}^{+}\right) \mathrm{m} / z$ calculated for $\mathrm{C}_{18} \mathrm{H}_{32} \mathrm{NaO}\left[\mathrm{M}+\mathrm{Na}^{+}\right]$: 287.2345; found: 287.2346.

$(1 S, 2 R, 9 R, E)-2-i s o-b u t y l-6,10,10-t r i m e t h y l b i c y c l o[7.2 .0]$ undec-5-en-2-ol (33)

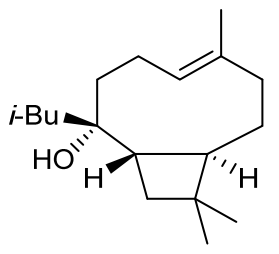

Compound $\mathbf{3 3}$ was synthesised according to the General Procedure A using ketone 22 ( $0.90 \mathrm{mmol}, 196$ $\mathrm{mg}$ ) and $2.0 \mathrm{M}$ iso-butylmagnesium bromide in $\mathrm{Et}_{2} \mathrm{O}$ for $2 \mathrm{~h}$. Flash chromatography was performed using pentane/ $\mathrm{Et}_{2} \mathrm{O}(15: 1)$ as mobile phase. It was isolated as a colourless oil. NMR spectra of this compound exhibit broad peaks at room temperature due to slow interconversion of conformers. The VT- ${ }^{1} \mathrm{H}$ NMR and ${ }^{13} \mathrm{C}$ NMR spectra were measured at $100{ }^{\circ} \mathrm{C}$.

Yield: $225 \mathrm{mg}, 90 \%$.

$\mathbf{R}_{f} 0.5$ (pentane / $\left.\mathrm{Et}_{2} \mathrm{O}, 9: 1\right)$

IR $v_{\max }\left(\right.$ thin film) $/ \mathrm{cm}^{-1} 3618 \mathrm{br}, 2950 \mathrm{~s}, 2932 \mathrm{~s}, 2856 \mathrm{~s}, 1449 \mathrm{~m}, 1365 \mathrm{~m}, 1280 \mathrm{~m}, 1187 \mathrm{~m}, 1036 \mathrm{~m}, 948 \mathrm{~m}$, $882 \mathrm{~m}$

${ }^{1}$ H NMR $\left(500 \mathrm{MHz}\right.$, Toluene-d $\left.\mathrm{d}_{8}, 373 \mathrm{~K}\right) \delta 5.44(\mathrm{t}, J=8.0 \mathrm{~Hz}, 1 \mathrm{H}), 2.17(\mathrm{~m}, 1 \mathrm{H}), 2.09-2.05(\mathrm{~m}, 1 \mathrm{H})$, $1.85(\mathrm{td}, J=12.8,4.6 \mathrm{~Hz}, 1 \mathrm{H}), 1.81-1.74(\mathrm{~m}, 2 \mathrm{H}), 1.73-1.66(\mathrm{~m}, 3 \mathrm{H}), 1.63-1.60(\mathrm{~m}, 1 \mathrm{H}), 1.58(\mathrm{~s}$, $3 \mathrm{H}) 1.51-1.30(\mathrm{~m}, 4 \mathrm{H}) 1.19-1.13(\mathrm{~m}, 2 \mathrm{H}), 0.98(\mathrm{~d}, \mathrm{~J}=6.6 \mathrm{~Hz}, 3 \mathrm{H}), 0.95(\mathrm{~s}, 3 \mathrm{H}), 0.92(\mathrm{~s}, 3 \mathrm{H}), 0.90$ $(\mathrm{d}, J=6.7 \mathrm{~Hz}, 3 \mathrm{H})$.

${ }^{13}$ C NMR (126 MHz, Toluene-d 8 , 373K) $\delta$ 134.8, 75.6, 53.2, 51.9, 47.8, 40.1, 39.7, 38.1, 32.2, 31.1, $30.1,25.6,25.2,24.9,23.8,23.6,17.1$.

NOTE: In the ${ }^{13} \mathrm{C}$ NMR, the disubstituted alkene resonance (125.0) is hidden under the solvent residual signal of toluene- $\mathrm{d}_{8}(\mathrm{t}, 125.5 \mathrm{ppm})$ as can be seen in the HSQC spectrum.

HRMS $\left(\mathrm{ESI}^{+}\right) \mathrm{m} / \mathrm{z}$ calculated for $\mathrm{C}_{18} \mathrm{H}_{32} \mathrm{NaO}\left[\mathrm{M}+\mathrm{Na}^{+}\right]: 287.2345$; found: 287.2343.

$(1 S, 2 R, 9 R, E)-2-h e x y l-6,10,10-t r i m e t h y l b i c y c l o[7.2 .0]$ undec-5-en-2-ol (35)

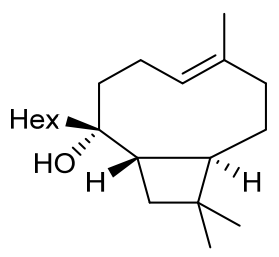

Compound 35 was synthesised according to the General Procedure A using ketone $22(0.485 \mathrm{mmol}$, $100 \mathrm{mg}$ ) and $0.8 \mathrm{M}$ hexylmagnesium bromide in THF for $2 \mathrm{~h}$. Flash chromatography was performed using pentane/ $\mathrm{Et}_{2} \mathrm{O}$ (9:1) as mobile phase. It was isolated as a colourless oil. NMR spectra of this compound exhibit broad peaks at room temperature due to slow interconversion of conformers. The VT- ${ }^{1} \mathrm{H}$ NMR and ${ }^{13} \mathrm{C}$ NMR spectra were measured at $100{ }^{\circ} \mathrm{C}$. 
Yield: $58 \mathrm{mg}, 68 \%$.

$\mathbf{R}_{f} 0.32$ (pentane / $\mathrm{Et}_{2} \mathrm{O}, 9: 1$ )

IR $v_{\max }\left(\right.$ thin film) $/ \mathrm{cm}^{-1} 3493 \mathrm{br}, 2950 \mathrm{~s}, 2923 \mathrm{~s}, 2854 \mathrm{~s}, 1459 \mathrm{~m}, 1366 \mathrm{~m}, 1258 \mathrm{~m}, 1083 \mathrm{~m}, 988 \mathrm{~m}, 877 \mathrm{~m}$

${ }^{1}$ H NMR $(500 \mathrm{MHz}$, Toluene-d $8,373 \mathrm{~K}) \delta 5.46(\mathrm{t}, J=8.0 \mathrm{~Hz}, 1 \mathrm{H}), 2.18-2.14(\mathrm{~m}, 1 \mathrm{H}) 2.07(\mathrm{~d}, J=12.3$ $\mathrm{Hz}, 1 \mathrm{H}), 1.90-1.79(\mathrm{~m}, 2 \mathrm{H}), 1.77-1.63(\mathrm{~m}, 3 \mathrm{H}), 1.59(\mathrm{~s}, 3 \mathrm{H}), 1.58-1.42(\mathrm{~m}, 4 \mathrm{H}), 1.36-1.20$ (m, $9 \mathrm{H}), 1.16(\mathrm{~d}, J=8.0 \mathrm{~Hz}, 2 \mathrm{H}), 0.96(\mathrm{~s}, 3 \mathrm{H}), 0.93(\mathrm{~s}, 3 \mathrm{H}), 0.91-0.88(\mathrm{~m}, 3 \mathrm{H})$.

${ }^{13}$ C NMR (126 MHz, Toluene-d $\left.\mathrm{d}_{8}, 373 \mathrm{~K}\right) \delta 134.8,74.7,52.0,47.8,44.7,40.1,37.7,32.5,32.3,31.2$, $30.9,30.1,27.6,24.7,23.7,23.5,23.2,17.1,14.3$.

NOTE: In the ${ }^{13} \mathrm{C}$ NMR, the disubstituted alkene resonance (125.2) is hidden under the solvent residual signal of toluene- $\mathrm{d}_{8}(\mathrm{t}, 125.5 \mathrm{ppm})$ as can be seen in the HSQC spectrum.

HRMS $\left(\mathrm{ESI}^{+}\right) \mathrm{m} / \mathrm{z}$ calculated for $\mathrm{C}_{20} \mathrm{H}_{36} \mathrm{NaO}\left[\mathrm{M}+\mathrm{Na}^{+}\right]$: 315.2658 ; found: 315.2653 .

$(1 S, 2 R, 9 R, E)-2-o c t y l-6,10,10-t r i m e t h y l b i c y c l o[7.2 .0]$ undec-5-en-2-ol (37)

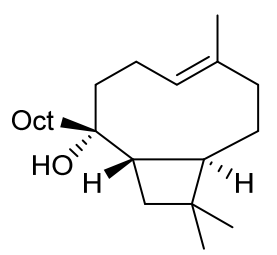

Compound 37 was synthesised according to the General Procedure B using ketone $22(0.485 \mathrm{mmol}$, $100 \mathrm{mg}$ ) and $0.23 \mathrm{M}$ octylmagnesium bromide in THF for $2.5 \mathrm{~h}$. The reaction mixture of the preparation of the Grignard reagent had to be refluxed for $2 \mathrm{~h}$ in order to obtain the full conversion to reagent. Flash chromatography performed using cyclohexane/ $\mathrm{Et}_{2} \mathrm{O}(19: 1)$ as mobile phase. It was isolated as a colourless oil. NMR spectra of this compound exhibit broad peaks at room temperature due to slow interconversion of conformers. The VT- ${ }^{1} \mathrm{H}$ NMR and ${ }^{13} \mathrm{C}$ NMR spectra were measured at $100{ }^{\circ} \mathrm{C}$.

Yield: $133 \mathrm{mg}, 84 \%$.

$\mathbf{R}_{f} 0.38$ (cyclohexane / $\mathrm{Et}_{2} \mathrm{O}, 9: 1$ )

IR $v_{\max }\left(\right.$ thin film) $/ \mathrm{cm}^{-1} 3506 \mathrm{br}, 2922 \mathrm{~s}, 2853 \mathrm{~s}, 1460 \mathrm{~m}, 1366 \mathrm{~m}, 1278 \mathrm{~m}, 1120 \mathrm{~m}, 1083 \mathrm{~m}, 1050 \mathrm{~m}, 989 \mathrm{~m}$, $941 \mathrm{~m}, 878 \mathrm{~m}$

${ }^{1}$ H NMR $(500 \mathrm{MHz}$, Toluene-d $8,373 \mathrm{~K}) \delta 5.47(\mathrm{t}, J=7.9 \mathrm{~Hz}, 1 \mathrm{H}), 2.19-2.14(\mathrm{~m}, 1 \mathrm{H}), 2.08(\mathrm{~d}, J=$ $12.8 \mathrm{~Hz}, 1 \mathrm{H}), 1.90-1.80(\mathrm{~m}, 2 \mathrm{H}), 1.78-1.64(\mathrm{~m}, 3 \mathrm{H}), 1.59(\mathrm{~s}, 3 \mathrm{H}), 1.59-1.41(\mathrm{~m}, 4 \mathrm{H}), 1.36-1.23$ $(\mathrm{m}, 13 \mathrm{H}), 1.21-1.14(\mathrm{~m}, 2 \mathrm{H}), 0.96(\mathrm{~s}, 3 \mathrm{H}), 0.93(\mathrm{~s}, 3 \mathrm{H}), 0.92-0.89(\mathrm{~m}, 3 \mathrm{H})$.

${ }^{13}$ C NMR (126 MHz, Toluene-d $\left.\mathrm{d}_{8}, 373 \mathrm{~K}\right) \delta 134.8,74.8,52.0,47.8,44.7,40.1,37.7,32.6,32.3,31.3$, $31.2,31.0,30.3,30.1,30.0,24.8,23.7,23.5,23.3,17.2,14.3$.

NOTE: In the ${ }^{13} \mathrm{C}$ NMR, the disubstituted alkene resonance (125.2) is hidden under the solvent residual signal of toluene-d8 ( $t, 125.5 \mathrm{ppm})$ as can be seen in the HSQC spectrum.

HRMS $\left(\mathrm{ESI}^{+}\right) \mathrm{m} / \mathrm{z}$ calculated for $\mathrm{C}_{22} \mathrm{H}_{40} \mathrm{NaO}\left[\mathrm{M}+\mathrm{Na}^{+}\right]: 343.2971$; found: 343.2965 . 


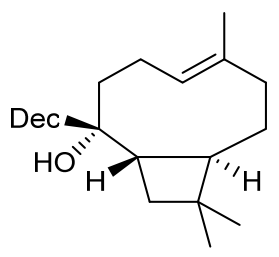

Compound 39 was synthesised according to the General Procedure B using ketone $22(0.339 \mathrm{mmol}, 70$ $\mathrm{mg}$ ) and $0.73 \mathrm{M}$ octylmagnesium bromide in THF for $64 \mathrm{~h}$. The reaction mixture of the preparation of the Grignard reagent had to be refluxed for $3 \mathrm{~h}$ in order to obtain the full conversion to reagent. Flash chromatography performed using cyclohexane/ $\mathrm{Et}_{2} \mathrm{O}(19: 1)$ as mobile phase. It was isolated as a colourless oil. NMR spectra of this compound exhibit broad peaks at room temperature due to slow interconversion of conformers. The VT- ${ }^{1} \mathrm{H}$ NMR and ${ }^{13} \mathrm{C}$ NMR spectra were measured at $100{ }^{\circ} \mathrm{C}$.

Yield: $106 \mathrm{mg}, 89 \%$.

$\mathbf{R}_{\boldsymbol{f}} 0.39$ (cyclohexane / $\mathrm{Et}_{2} \mathrm{O}, 9: 1$ )

IR $v_{\max }\left(\right.$ thin film) $/ \mathrm{cm}^{-1} 3484 \mathrm{br}, 2921 \mathrm{~s}, 2852 \mathrm{~s}, 1460 \mathrm{~m}, 1366 \mathrm{~m}, 1277 \mathrm{~m}, 1120 \mathrm{~m}, 1083 \mathrm{~m}, 1041 \mathrm{~m}, 988 \mathrm{~m}$, 944m, $877 \mathrm{~m}$

${ }^{1} \mathbf{H}$ NMR $(500 \mathrm{MHz}$, Toluene-d $8,373 \mathrm{~K}) \delta 5.47(\mathrm{t}, J=8.0 \mathrm{~Hz}, 1 \mathrm{H}), 2.15(\mathrm{~m}, 1 \mathrm{H}), 2.08(\mathrm{~d}, J=12.8 \mathrm{~Hz}$, $1 \mathrm{H}), 1.90-1.80(\mathrm{~m}, 2 \mathrm{H}), 1.78-1.65(\mathrm{~m}, 3 \mathrm{H}), 1.59(\mathrm{~s}, 3 \mathrm{H}), 1.58-1.38(\mathrm{~m}, 4 \mathrm{H}), 1.38-1.23(\mathrm{~m}, 17 \mathrm{H})$, $1.19(\mathrm{~d}, J=6.4 \mathrm{~Hz}, 2 \mathrm{H}), 0.96(\mathrm{~s}, 3 \mathrm{H}), 0.93(\mathrm{~s}, 3 \mathrm{H}), 0.90$ (t, $J=6.8 \mathrm{~Hz}, 3 \mathrm{H})$.

${ }^{13}$ C NMR (126 MHz, Toluene-d 8 , 373K) $\delta 134.8,74.6,52.0,47.8,44.7,40.1,40.0,37.7,32.6,32.3$, $31.3,31.2,30.4,30.4,30.3,30.1,30.0,24.8,23.7,23.5,23.3,17.2,14.3$.

NOTE: In the ${ }^{13} \mathrm{C}$ NMR, the disubstituted alkene resonance (125.2) is hidden under the solvent residual signal of toluene- $\mathrm{d}_{8}(\mathrm{t}, 125.5 \mathrm{ppm})$ as can be seen in the HSQC spectrum.

HRMS $\left(\mathrm{ESI}^{+}\right) \mathrm{m} / \mathrm{z}$ calculated for $\mathrm{C}_{24} \mathrm{H}_{44} \mathrm{NaO}\left[\mathrm{M}+\mathrm{Na}^{+}\right]$: 371.3282 ; found: 371.3284 .

\section{Cyclization studies}

\section{Screening scale reactions}

Resorcinarene 1 (11.1 mg, $1.67 \mu \mathrm{mol}, 0.10$ eq. capsule I) was dissolved in $200 \mu \mathrm{L}$ of water-satd. chloroform. To this solution was added a $n$-decane stock solution in water-satd. chloroform $(20 \mu \mathrm{L}, 167$ $\mathrm{mmol}^{-1}, 3.34 \mu \mathrm{mol}, 0.2 \mathrm{eq}$ ). Additional water-satd. chloroform was added to bring the total volume to $500 \mu \mathrm{L}$.

The substrate 23 was then added ( $3.61 \mathrm{mg}, 16.7 \mu \mathrm{mol}, 1.00 \mathrm{eq})$ followed by an $\mathrm{HCl}$ stock solution in chloroform $(0.50 \mu \mathrm{mol}, 0.03 \mathrm{eq})$, and the mixture was briefly stirred.

An aliquot (approximately $10 \mu \mathrm{L}$ ) of the reaction mixture was diluted with $0.25 \mathrm{~mL}$ of hexane (containing $0.08 \%$ DMSO) and subjected, after centrifugation to remove the precipitate formed, to gas chromatographic analysis (initial sample).

The reaction mixture was then stirred at $30{ }^{\circ} \mathrm{C}$ (internal temperature), and further samples were taken at the indicated times and analyzed by gas chromatography. Conversions and yields were calculated as described in our previous work. ${ }^{4}$ 


\section{Preparation and titration of $\mathrm{HCl}$ stock solution in chloroform}

$\mathrm{HCl}$ stock solution in chloroform was prepared by passing $\mathrm{HCl}$ gas, generated by the dropwise addition of concentrated $\mathrm{H}_{2} \mathrm{SO}_{4}$ to dry $\mathrm{NaCl}$, through chloroform for approximately 30 mins. The concentration of $\mathrm{HCl}$ in the resulting solution was determined as follows: $\mathrm{HCl}$ stock solution in chloroform $(100 \mu \mathrm{L})$ was added to a solution of phenol red in EtOH $(0.002 \mathrm{wt} \%, 2.5 \mathrm{~mL})$ via a Microman M1 pipette equipped with plastic tips. Upon addition the solution turned from yellow (neutral) to pink (acidic). The resulting solution was then titrated with a $0.1 \mathrm{M}$ ethanolic solution of triethylamine. At the equivalence point the solution turned from pink to yellow. The $\mathrm{HCl}$ stock solution was kept in the fridge, and the titration was repeated immediately before each use.

\section{Preparation of water-satd. chloroform}

Chloroform $(10 \mathrm{~mL})$ was filtered through a plug of aluminum oxide (basic, Brockmann grade I). To this chloroform was added distilled water $(1 \mathrm{~mL})$, the mixture was sonicated for approximately $5 \mathrm{~min}$ then left standing until complete phase separation. Water-satd. chloroform was prepared freshly for each use. 
Solvent screening results

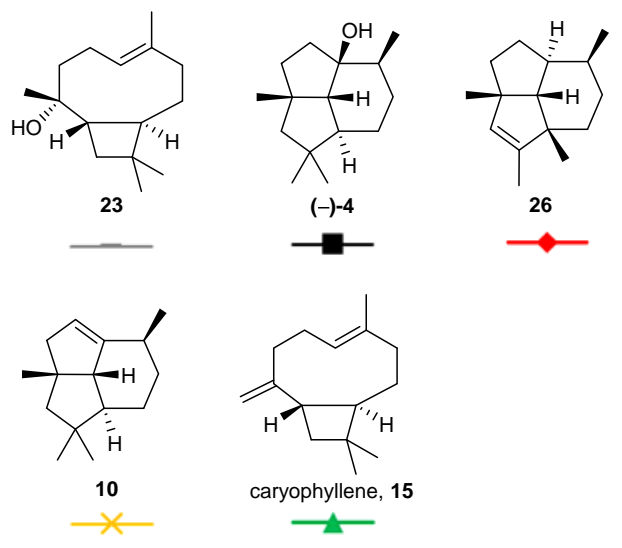

Chloroform, $3 \mathrm{~mol} \% \mathrm{HCl}$

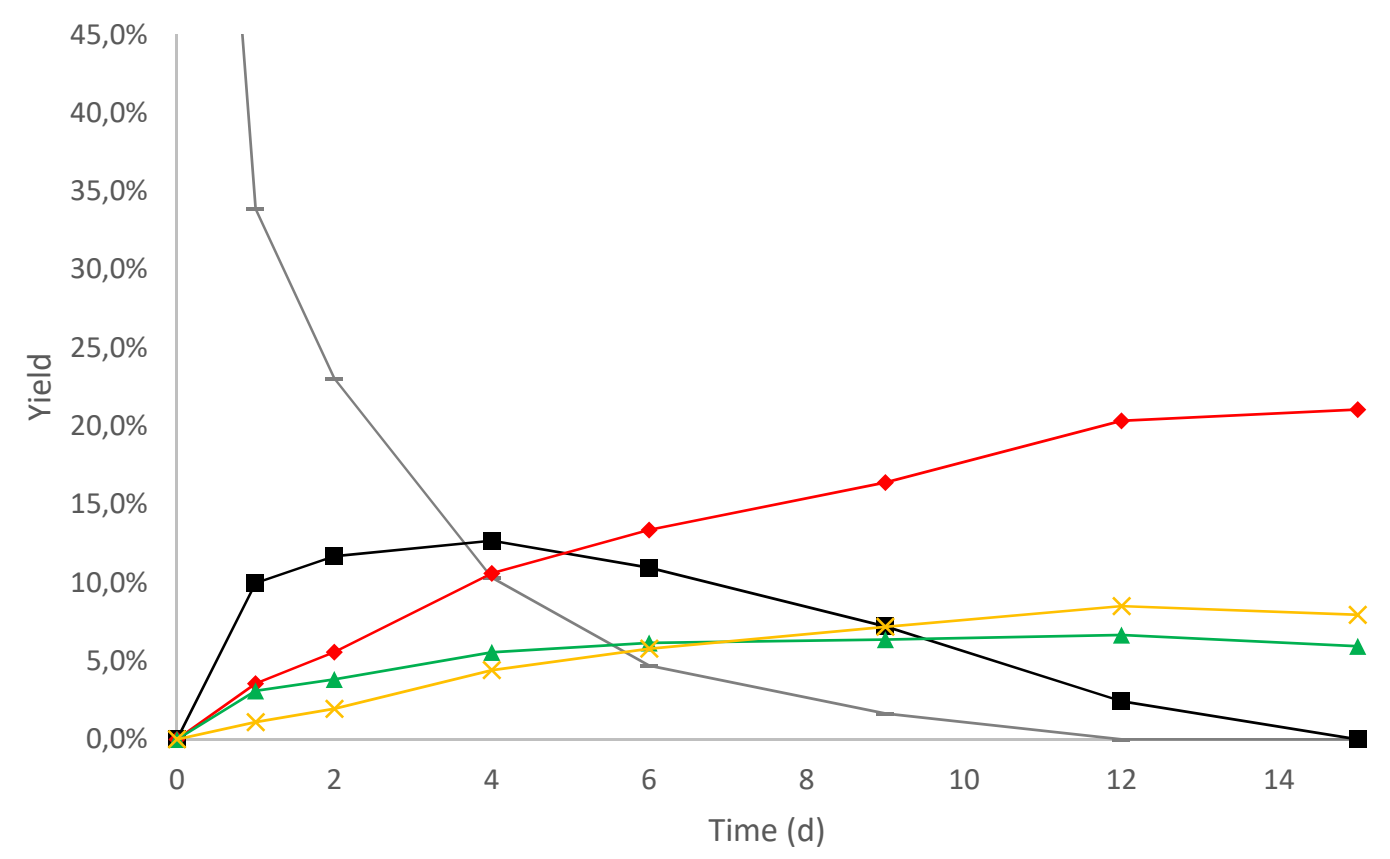



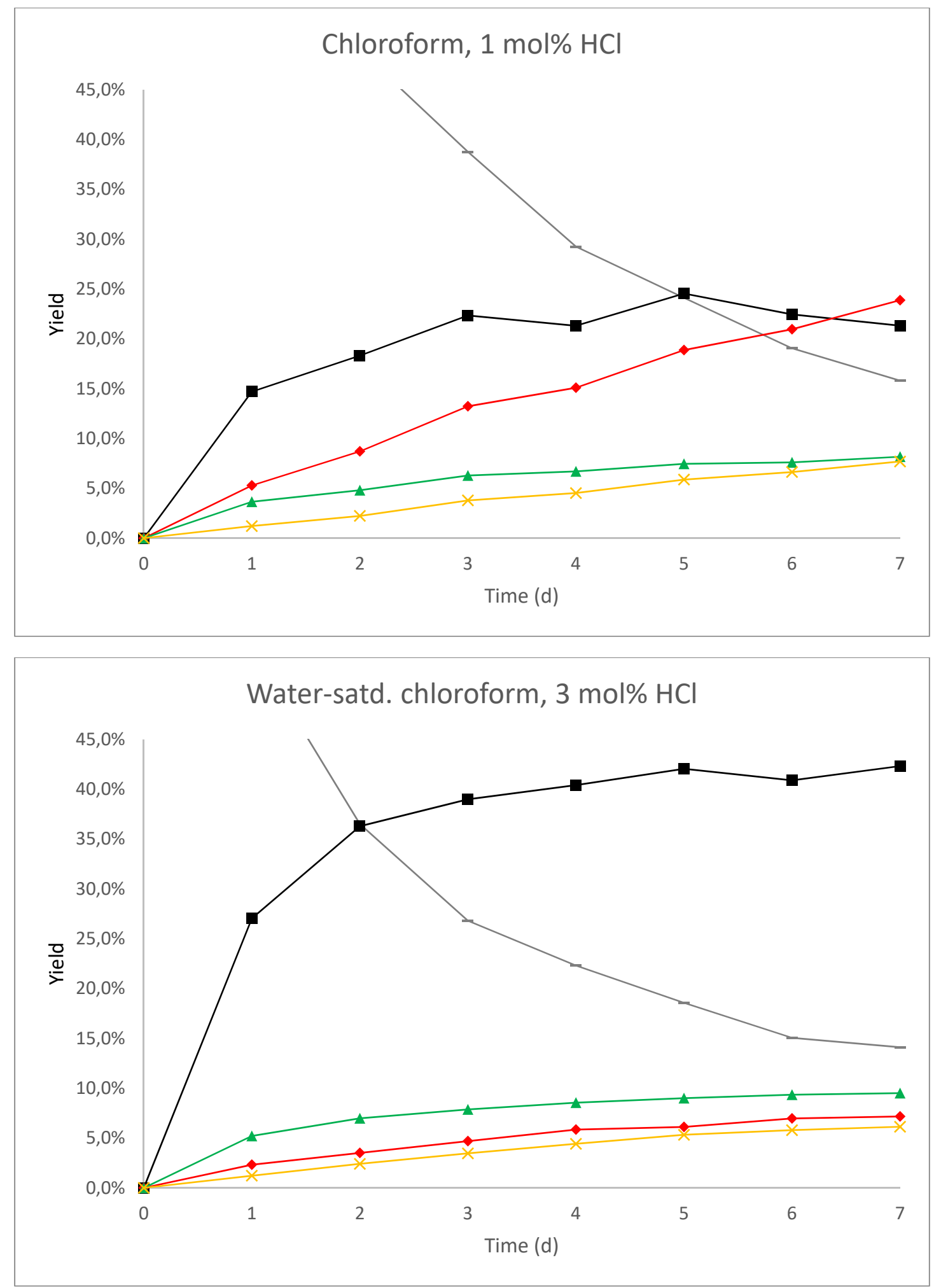

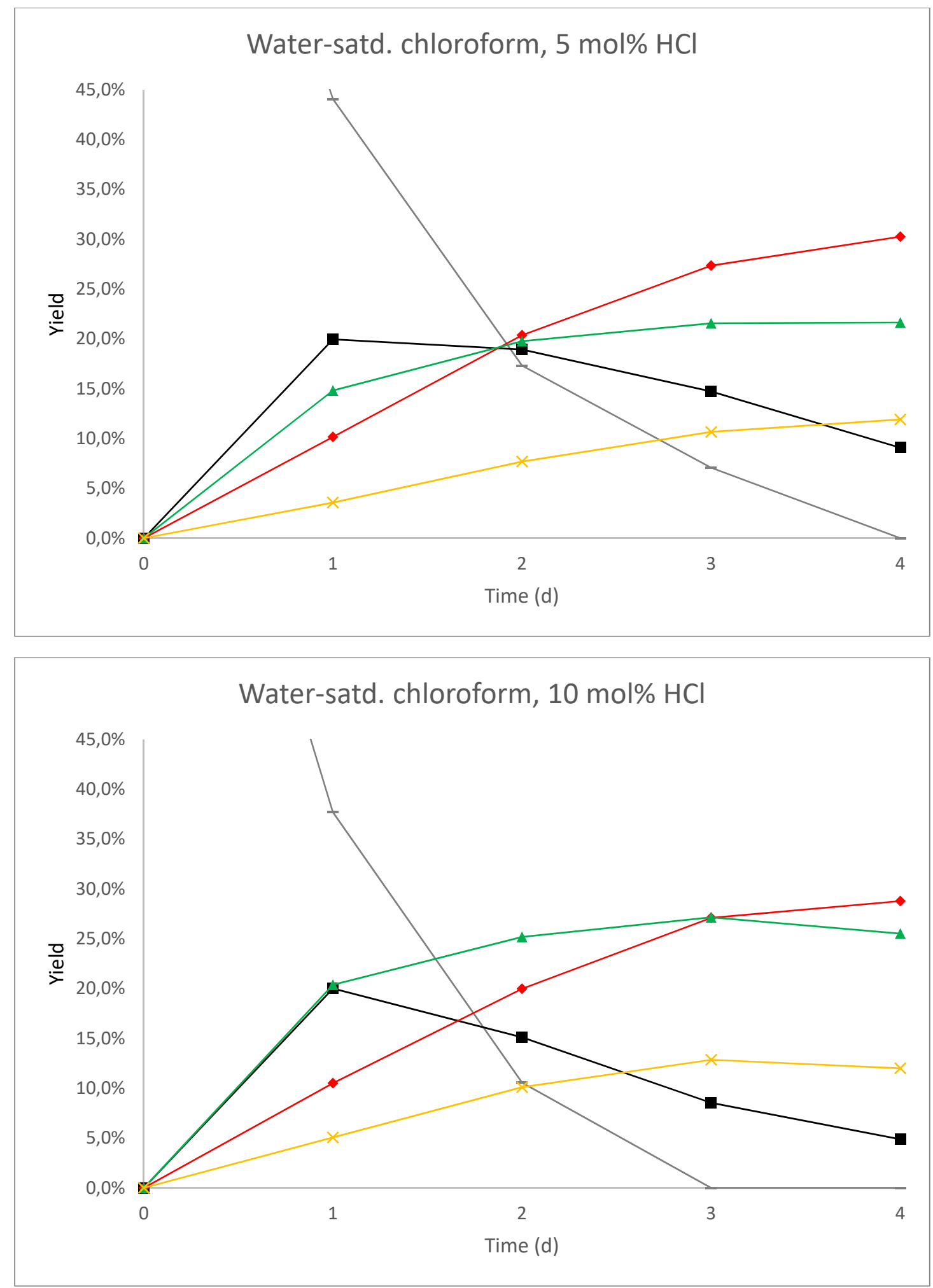

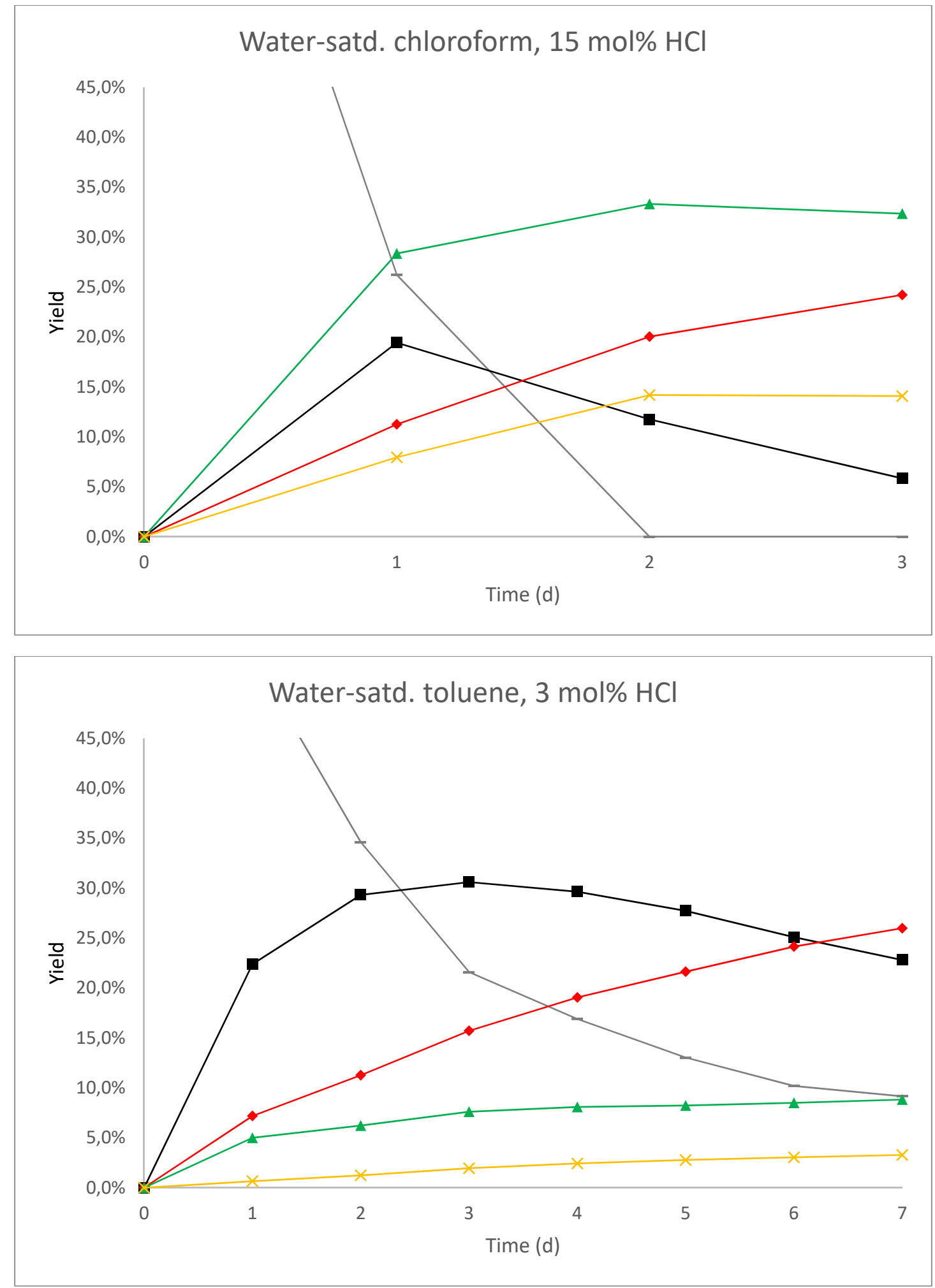

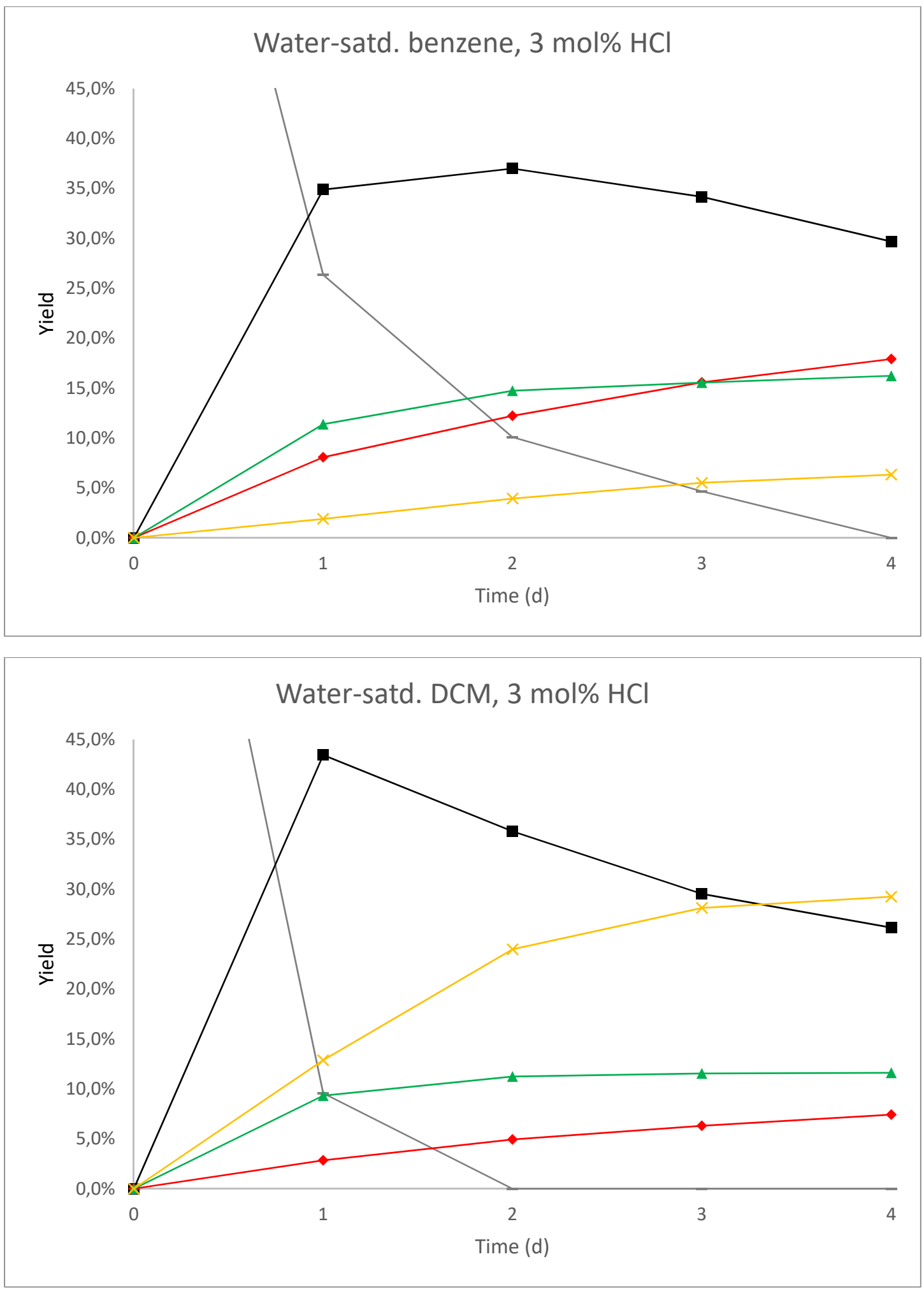


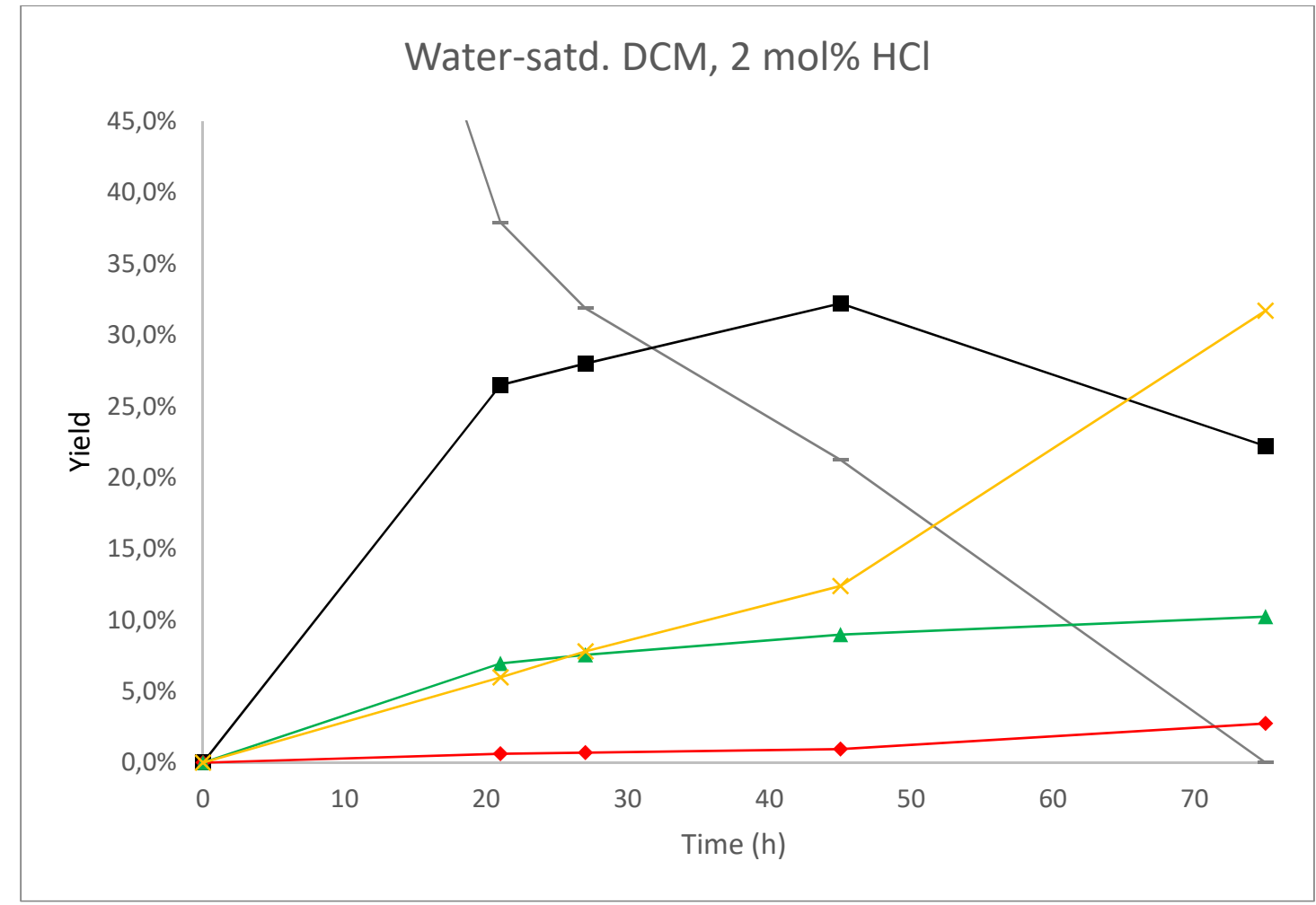

\section{GC traces of selected reaction conditions}

a) normal $\mathrm{CHCl}_{3}, 3 \mathrm{~mol} \% \mathrm{HCl}$, after $12 \mathrm{~d}$

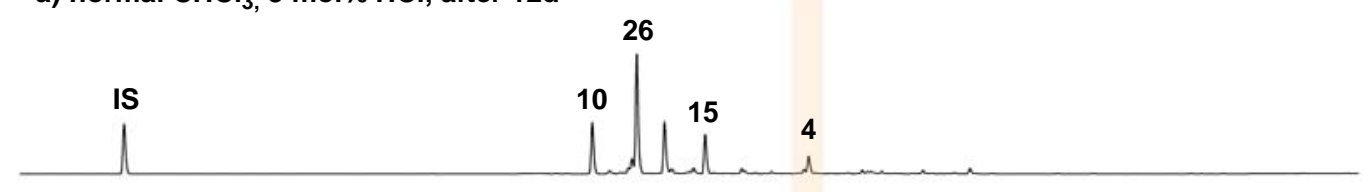

b) water-satd. $\mathrm{CHCl}_{3}, 3 \mathrm{~mol} \% \mathrm{HCl}$, after $7 \mathrm{~d}$

23

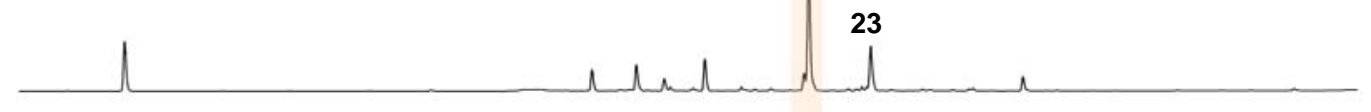

c) water-satd. DCM, 3 mol\% $\mathrm{HCl}$, after $2 \mathrm{~d}$

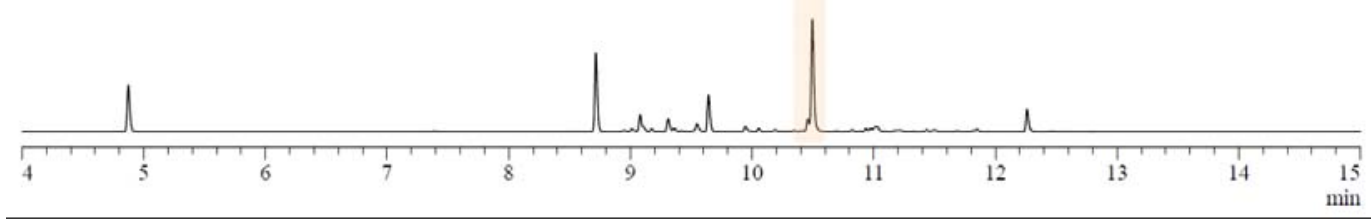

Figure S-1: GC traces of selected reaction conditions, IS: internal standard (decane)

\section{Control experiments}

Control experiments were carried out to elucidate the role of the resorcinarene capsule $\mathbf{I}$ and $\mathrm{HCl}$ for catalytic activity. When the reaction was performed in water-satd. chloroform with $3 \mathrm{~mol} \% \mathrm{HCl}$ in the 
absence of capsule at $30^{\circ} \mathrm{C}$, the only reaction observed was the slow formation of caryophyllene (see $\mathrm{GC}$ trace below). When the $\mathrm{HCl}$ was omitted and the reaction was carried out under otherwise standard conditions $\left(10 \mathrm{~mol} \%\right.$ capsule $\mathbf{I}$, water-satd. chloroform as the solvent, $\left.30^{\circ} \mathrm{C}\right)$, no conversion was observed. Finally, when the capsule's cavity was blocked with a strongly-binding ligand (1.5 eq of $\mathrm{Bu}_{4} \mathrm{NBr}$ per capsule, otherwise standard conditions) only conversion to caryophyllene was observed. These results taken together provide strong evidence that the reaction takes place inside the cavity of capsule I, and that it relies on the synergistic activity between the capsule and $\mathrm{HCl}$.

a) reaction without capsule and with $\mathrm{HCl}$ after $5 \mathrm{~d}$

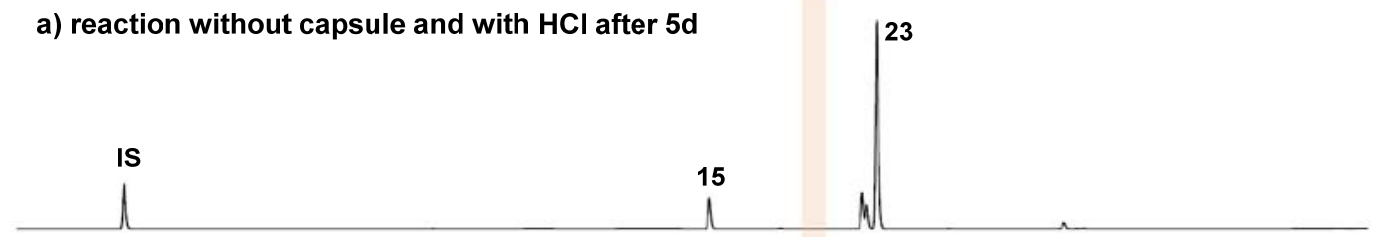

b) reaction with capsule and without $\mathrm{HCl}$ after $4 \mathrm{~d}$

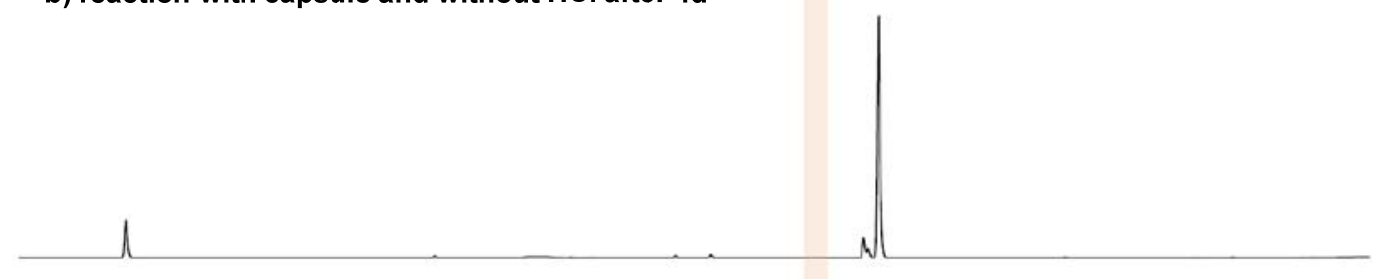

c) reaction with blocked capsule and with $3 \mathrm{~mol} \%$ of $\mathrm{HCl}$ after $4 \mathrm{~d}$

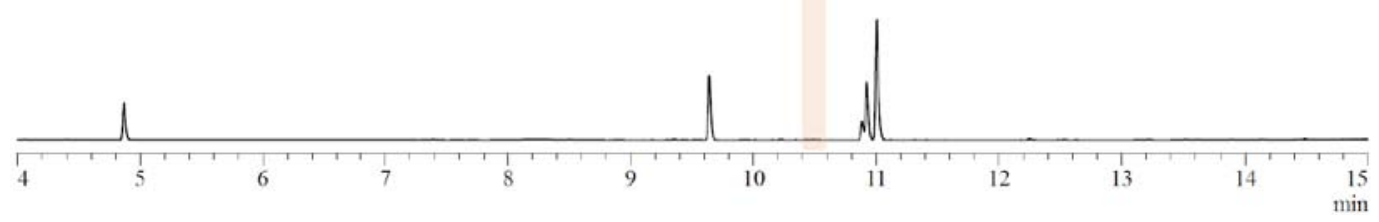

Figure S-2: Control experiments using substrate $\mathbf{2 3}$ under various modified reaction conditions.

Additionally, control experiments were performed in which presilphiperfolan-1 $1 \beta$-ol was resubjected to the reaction conditions in the absence of capsule $\mathbf{I}$. When either untreated chloroform or water-satd. DCM was used as the solvent, only slow formation of small quantities of elimination product $\mathbf{1 0}$ and of rearranged alkene 20 was observed (approximately $10 \%$ and 3.5\% GC yield, respectively after $9 \mathrm{~d}$ ), therefore indicating that the formation of rearrangement product $\mathbf{2 0}$ and of elimination product $\mathbf{1 0}$ during reaction in the respective solvents is a capsule mediated process.

Full list of control experiments performed using substrate (SM) and conditions reported in literature for terpene cyclisation ${ }^{3,5}$ 
a) reaction with capsule I and $3 \mathrm{~mol} \% \mathrm{HCl}$ after $6 \mathrm{~d}$

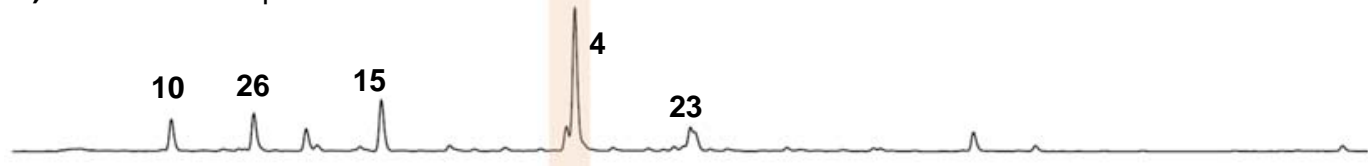

b) $\mathrm{BF}_{3 .} \mathrm{Et}_{2} \mathrm{O}, \mathrm{Et}_{2} \mathrm{O}, 0{ }^{\circ} \mathrm{C}, 0.5 \mathrm{~h}$ (ref. $5 \mathrm{a}$ )

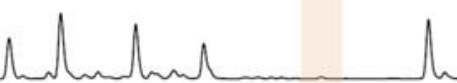

c) $\mathrm{BCl}_{3}, \mathrm{DCM},-80^{\circ} \mathrm{C}, 4 \mathrm{~h}$ (ref. $5 \mathrm{~b}$ )

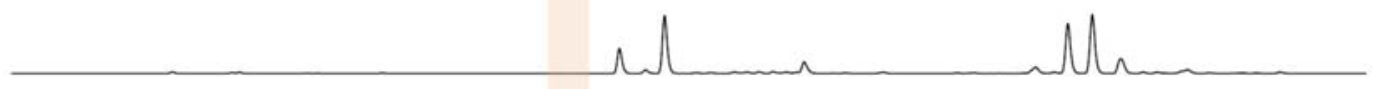

d) $\mathrm{BBr}_{3}, \mathrm{DCM},-22^{\circ} \mathrm{C}, 2 \mathrm{~h}$ (ref. $5 b$ )

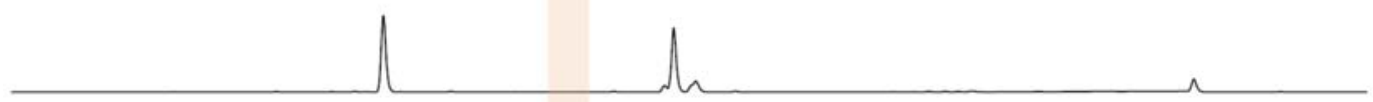

e) $\mathrm{SOCl}_{2}$, pyr, $0^{\circ} \mathrm{C}-20^{\circ} \mathrm{C}, 1 \mathrm{~h}$ (ref. 5c)

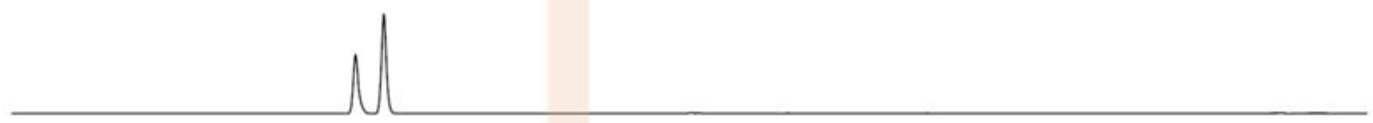

f) pyr, $60 \%$ aq. acetone, $125^{\circ} \mathrm{C}, 2 \mathrm{~h}$ (ref. 3)

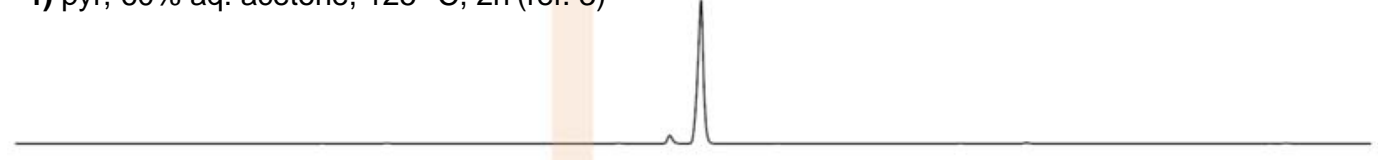

g) $\mathrm{HCOOH}, 0{ }^{\circ} \mathrm{C}, 2 \mathrm{~h}$ (ref. $5 \mathrm{~d}$ )

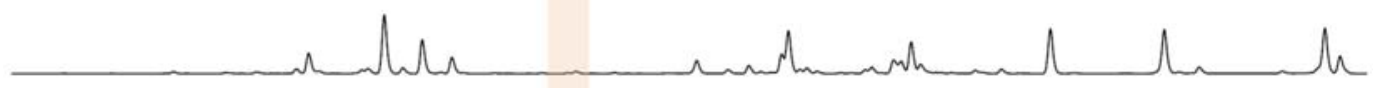

h) $\mathrm{HCOOH}, 50^{\circ} \mathrm{C}, 0.5 \mathrm{~h}$ (ref. $5 \mathrm{~d}$ )

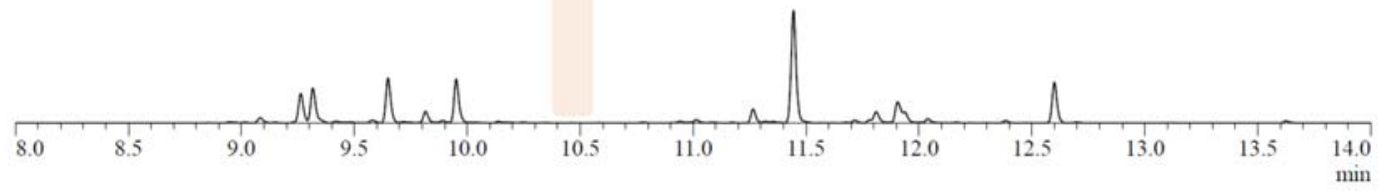

Figure S-3: Control experiments using substrate 23 and various literature reported conditions for terpene cyclisations. 
a) reaction with capsule I and $3 \mathrm{~mol} \% \mathrm{HCl}$ after $6 \mathrm{~d}$

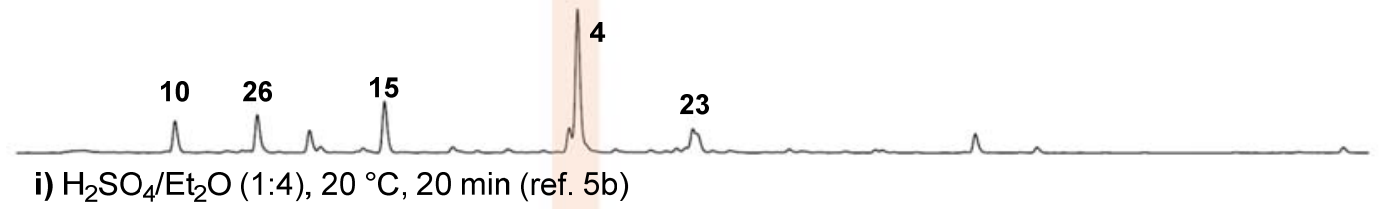

i) $\mathrm{H}_{2} \mathrm{SO}_{4} / \mathrm{Et}_{2} \mathrm{O}(1: 4), 20{ }^{\circ} \mathrm{C}, 20 \mathrm{~min}$ (ref. $5 \mathrm{~b}$ )

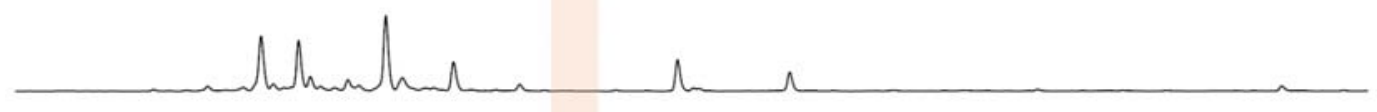

j) $\mathrm{H}_{2} \mathrm{SO}_{4} / \mathrm{THF}(1: 4), 20^{\circ} \mathrm{C}, 20 \mathrm{~min}$ (ref. $5 \mathrm{~b}$ )

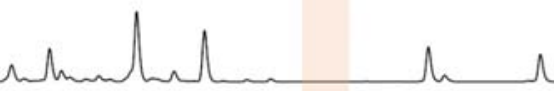

k) $\mathrm{H}_{2} \mathrm{SO}_{4}$ /acetone (1:4), $20^{\circ} \mathrm{C}, 20 \mathrm{~min}$ (ref. 5b)

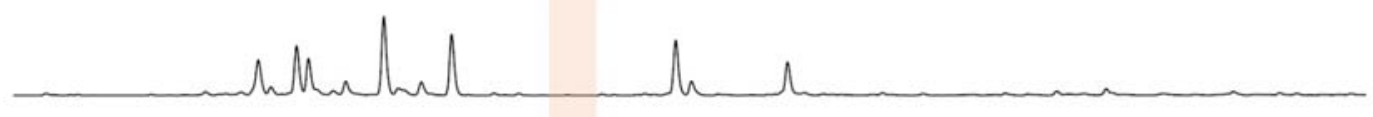

I) $\mathrm{H}_{2} \mathrm{SO}_{4} / \mathrm{DCM}$ (1:4), $20^{\circ} \mathrm{C}, 20 \mathrm{~min}$ (ref. 5b)

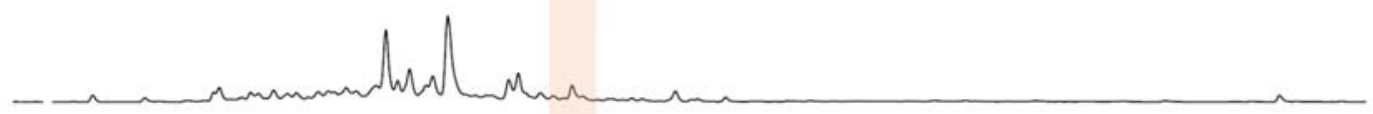

m) $40 \%$ aq. $\mathrm{H}_{2} \mathrm{SO}_{4}, \mathrm{THF}, 0^{\circ} \mathrm{C}-20^{\circ} \mathrm{C}, 2 \mathrm{~h}$ (ref. $5 \mathrm{e}$ )

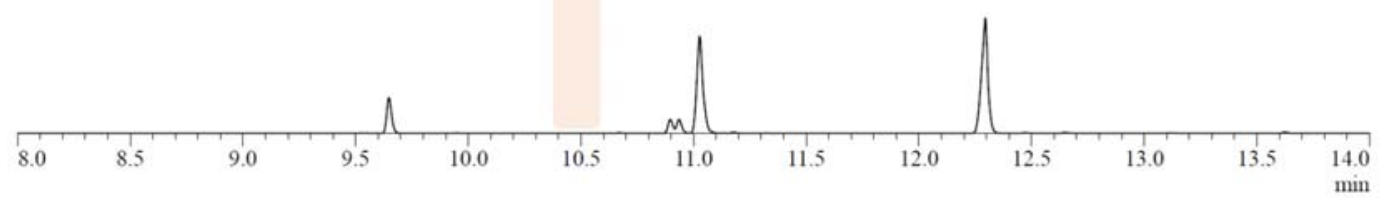

Figure S-4: Control experiments using substrate 23 and various literature reported conditions for terpene cyclisation.

a) NMR spectrum of (-)-4

b) NMR of crude reaction mixture

$\mathrm{H}_{2} \mathrm{SO}_{4} / \mathrm{DCM}(1: 4), 20^{\circ} \mathrm{C}$ - ently I)

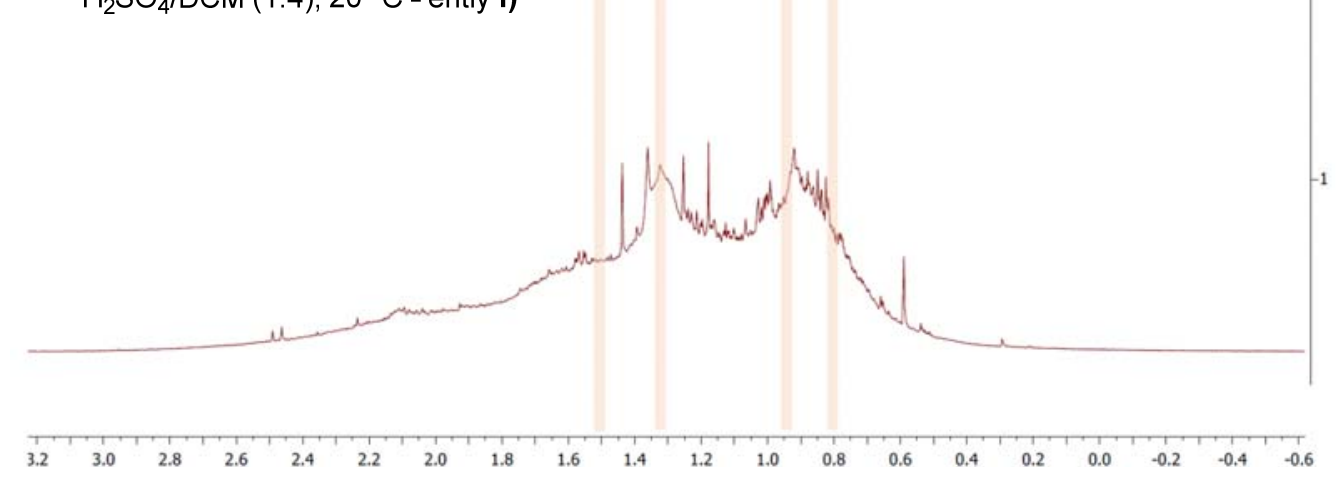

Figure S-5: Comparison of the NMR spectra of (-)-4 and crude NMR of the reaction (Figure 4, entry 1) 


\section{Reactions of caryophyllene}

To probe whether formation of key carbocation $\mathbf{6}$ directly from caryophyllene was possible, caryophyllene was subjected to reaction with $10 \mathrm{~mol} \%$ of capsule $\mathbf{I}$ and with $3 \mathrm{~mol} \%, 10 \mathrm{~mol} \%$ or 20 mol\% of $\mathrm{HCl}$ using chloroform as the solvent. At $30{ }^{\circ} \mathrm{C}$, no products were observed upon gas chromatographic analysis of the reaction mixture. Raising the temperature to $50{ }^{\circ} \mathrm{C}$ resulted in a complex reaction mixture, with the major products having retention times of $10.93,11.19$ and 11.50 min; alkene $\mathbf{2 6}$ was also observed in the mixture.

a) using 15 as starting substrate

$10 \mathrm{~mol} \%$ of capsule I, $10 \mathrm{~mol} \%$ of $\mathrm{HCl}, 30^{\circ} \mathrm{C}, 4 \mathrm{~d}$

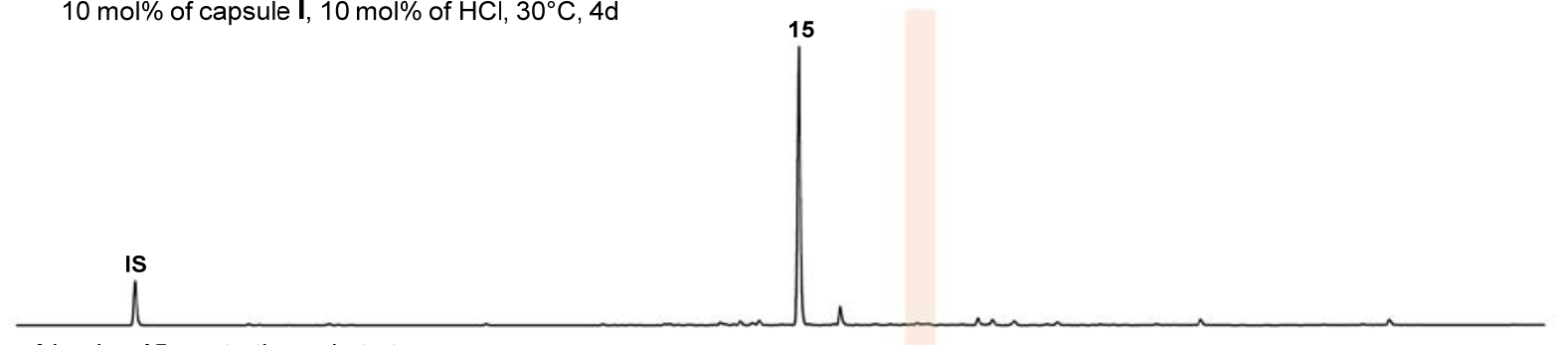

b) using 15 as starting substrate

$10 \mathrm{~mol} \%$ of capsule I, $3 \mathrm{~mol} \%$ of $\mathrm{HCl}, 50^{\circ} \mathrm{C}, 2 \mathrm{~d}$

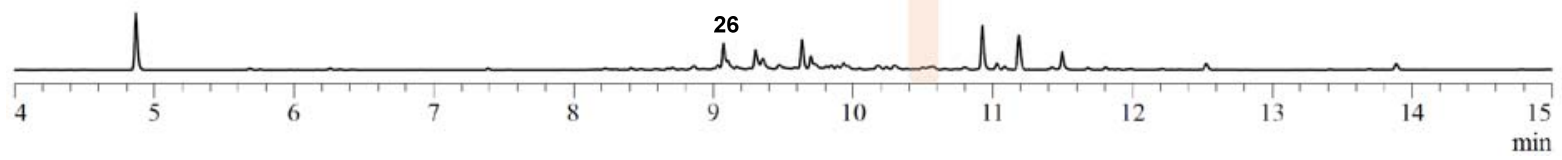

Figure S-6: Cyclisations using caryophyllene $\mathbf{1 5}$ as substrate, IS: internal standard (decane)

\section{Large scale reactions and isolation of products}

In order to obtain a high level of reproducibility while scaling up the reactions, we optimized the reaction setup for $33.5 \mu \mathrm{mol}(\mathrm{A}), 0.252 \mathrm{mmol}(\mathrm{B})$ and $0.503 \mathrm{mmol}$ (C) scale (scale is in regards to the amount of SM used).

For (A)-scale reactions, we set up the reaction in $1.5 \mathrm{~mL}$ GC vials (vials: 548-0028, caps: 548-0006, both ordered from VWR), which were filled with the reaction mixture to $2 / 3$ of their volume.

For (B)- and (C)-scale reactions, we used $10 \mathrm{~mL}$ vials (vials: 548-1393, caps: 548-1393, both ordered from VWR) and $20 \mathrm{~mL}$ vial (G075W-27/057-H, $20 \mathrm{~mL}$ glass vials with PP-screw cap and PTFEsealing), respectively. $3 / 4$ of their volume were filled with the reaction mixture. The vials were sealed with Teflon tape. If the reaction was performed on larger scale, pressure tubes were used ( $3 / 4$ filled).

\section{General procedure C: Large scale cyclization reactions using resorcinarene capsule and} $3 \mathrm{~mol} \%$ of $\mathrm{HCl}$

Resorcinarene 1 (167 mg, $0.151 \mathrm{mmol}, 0.10$ eq. capsule I) was dissolved in $3 \mathrm{~mL}$ of water-satd. chloroform. To this solution was added a $n$-decane stock solution in water-satd. chloroform $(302 \mu \mathrm{L}$, $\left.167 \mathrm{mmol} \mathrm{l}^{-1}, 50.4 \mu \mathrm{mol}, 0.2 \mathrm{eq}\right)$. 
The substrate $(0.252 \mathrm{mmol}, 1.00 \mathrm{eq})$ was added as its solution in water-satd. $\mathrm{CHCl}_{3}(2 \mathrm{ml}$, rinsing with $2 \times 1 \mathrm{~mL})$. Additional water-satd. chloroform was added to bring the total volume to $7.5 \mathrm{~mL}$.

Freshly titrated $\mathrm{HCl}$ stock solution in chloroform $(7.56 \mu \mathrm{mol}, 0.03 \mathrm{eq})$ was added, and the mixture was briefly stirred.

An aliquot (approximately $10 \mu \mathrm{L}$ ) of the reaction mixture was diluted with $0.25 \mathrm{~mL}$ of hexane (containing $0.08 \%$ DMSO) and subjected, after centrifugation to remove the precipitate formed, to gas chromatographic analysis (initial sample).

The reaction mixture was then stirred at $30{ }^{\circ} \mathrm{C}$ (internal temperature), and further samples were taken at the indicated times and analyzed by gas chromatography. Conversions and yields were calculated as described in our previous work. ${ }^{4}$

\section{Presilphiperfolan-1ß-ol [(-)-4]}

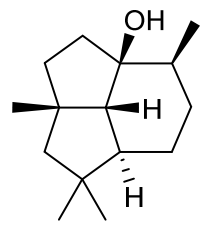

This compound was prepared on $1.8 \mathrm{mmol}$ scale using a $100 \mathrm{~mL}$ pressure tube. After $7 \mathrm{~d}$, a conversion of $90 \%$ was reached. At this point, the reaction mixture was directly loaded onto a column of silica (approximately $100 \mathrm{~g}$ ) and chromatographed, eluting first with pentane (to remove solvent, decane and nonpolar by-products) then with pentane / diethyl ether 9:1 to give the title compound as a white solid.

Yield: $139 \mathrm{mg}, 35 \%$

Optical rotation $[\alpha]_{\mathrm{D}}{ }^{25.0}-39.4\left(\mathrm{c} 0.63, \mathrm{CHCl}_{3}\right)$

$\mathbf{R}_{\boldsymbol{f}} 0.24$ (pentane / $\mathrm{Et}_{2} \mathrm{O}, 9: 1$ )

IR $v_{\max }\left(\right.$ thin film) $/ \mathrm{cm}^{-1} 3444 \mathrm{br}, 2927 \mathrm{~s}, 2862 \mathrm{~m}, 1455 \mathrm{~m}, 1364 \mathrm{w}, 1301 \mathrm{w}, 1257 \mathrm{w}, 1007 \mathrm{~m}, 996 \mathrm{w}, 945 \mathrm{~m}$

${ }^{1} \mathbf{H}$ NMR $\left(500 \mathrm{MHz}, \mathrm{C}_{6} \mathrm{D}_{6}\right) \delta 1.88-1.79(\mathrm{~m}, 2 \mathrm{H}), 1.72-1.65(\mathrm{~m}, 1 \mathrm{H}), 1.64-1.55(\mathrm{~m}, 2 \mathrm{H}), 1.56(\mathrm{~d}, J$ $=12.2 \mathrm{~Hz}, 1 \mathrm{H}), 1.50(\mathrm{~s}, 2 \mathrm{H}), 1.48-1.40(\mathrm{~m}, 1 \mathrm{H}), 1.36-1.26(\mathrm{~m}, 2 \mathrm{H}), 1.32(\mathrm{~s}, 3 \mathrm{H}), 1.18-1.07(\mathrm{~m}$, 2H), 0.94 (s, 3H), 0.90 (d, J=7.0 Hz, 3H), 0.80 (s, 3H), 0.61 (br s, 1H)

${ }^{13}$ C NMR $\left(125 \mathrm{MHz}, \mathrm{C}_{6} \mathrm{D}_{6}\right) \delta 84.5,63.9,59.4,51.7,46.2,42.7,40.5,39.7,36.9,31.4,29.9,28.8,22.3$, $20.3,15.7$

HRMS $\left(\mathrm{ESI}^{+}\right) \mathrm{m} / \mathrm{z}$ calculated for $\mathrm{C}_{15} \mathrm{H}_{26} \mathrm{NaO}\left[\mathrm{M}+\mathrm{Na}^{+}\right]: 245.1876$; found 245.1877

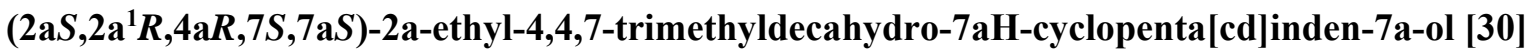

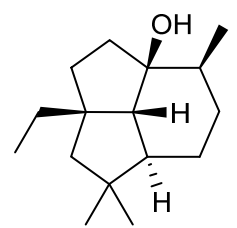

This compound was prepared on $0.252 \mathrm{mmol}$ scale (scale B). After $10 \mathrm{~d}$ a conversion of $85 \%$ was reached. At this point the reaction mixture was directly loaded onto a column of silica (approximately $40 \mathrm{~g}$ ) and chromatographed, eluting first with pentane (to remove solvent, decane and nonpolar byproducts) then with pentane / diethyl ether 9:1 to give the title compound as a colorless oil. 
Yield: $16.2 \mathrm{mg}, 27 \%$

$\mathbf{R}_{f} 0.21$ (pentane / $\mathrm{Et}_{2} \mathrm{O}, 9: 1$ )

IR $v_{\max }\left(\right.$ thin film) $/ \mathrm{cm}^{-1} 3458 \mathrm{br}, 2927 \mathrm{~s}, 2860 \mathrm{~m}, 1460 \mathrm{~m}, 1378 \mathrm{~m}, 1363 \mathrm{~m}, 974 \mathrm{~m}, 963 \mathrm{~m}$

${ }^{1} \mathbf{H}$ NMR $\left(400 \mathrm{MHz}, \mathrm{CDCl}_{3}\right) \delta 2.00(\mathrm{ddd}, J=13.2,9.0,7.6 \mathrm{~Hz}, 1 \mathrm{H}, \mathrm{H} 2), 1.95-1.80(\mathrm{~m}, 2 \mathrm{H}, \mathrm{H} 3, \mathrm{H} 9)$, $1.77-1.68$ (m, 1H, OH), 1.68 - 1.59 (m, 3H, H2, H5, H8), 1.56 - 1.37 (m, 4H, H2, H10, H1 1, H14), $1.33(\mathrm{~d}, J=13.4 \mathrm{~Hz}, 1 \mathrm{H}, \mathrm{H} 5), 1.30-1.17$ (m, 3H, H7, H10, H11), $0.99-0.90$ (m, 6H, 3x H13, 3x $\mathrm{H} 15), 0.88$ (t, J = 7.4 Hz, 3H, 3x H16), 0.80 (s, 3H, 3x H12).

${ }^{13} \mathrm{C}$ NMR $\left(101 \mathrm{MHz}, \mathrm{CDCl}_{3}\right) \delta 84.8\left(\mathrm{C}_{\mathrm{q}}, \mathrm{C} 1\right), 62.6(\mathrm{CH}, \mathrm{C} 8), 54.8\left(\mathrm{CH}_{2}, \mathrm{C} 5\right), 51.2(\mathrm{CH}, \mathrm{C} 7), 50.7\left(\mathrm{C}_{\mathrm{q}}\right.$, C4), $40.3\left(\mathrm{C}_{\mathrm{q}}, \mathrm{C} 6\right), 39.2\left(\mathrm{CH}_{2}, \mathrm{C} 2\right), 38.8\left(\mathrm{CH}_{2}, \mathrm{C} 3\right), 36.7(\mathrm{CH}, \mathrm{C} 9), 35.3\left(\mathrm{CH}_{2}, \mathrm{C} 14\right), 29.7\left(\mathrm{CH}_{2}, \mathrm{C} 10\right)$, $29.00\left(\mathrm{CH}_{3}, \mathrm{C} 13\right), 22.6\left(\mathrm{CH}_{3}, \mathrm{Cl}\right), 20.1\left(\mathrm{CH}_{2}, \mathrm{C} 11\right), 15.6\left(\mathrm{CH}_{3}, \mathrm{C} 15\right), 10.3\left(\mathrm{CH}_{3}, \mathrm{C} 16\right)$.

HRMS $\left(\mathrm{ESI}^{+}\right) \mathrm{m} / \mathrm{z}$ calculated for $\mathrm{C}_{16} \mathrm{H}_{27}\left[\mathrm{M}-\mathrm{OH}^{-}\right]: 219.2107$; found 219.2108

$\left(2 \mathrm{a} S, 2 \mathrm{a}^{1} \mathrm{R}, 4 \mathrm{a} R, 7 S, 7 \mathrm{a} S\right)-2 \mathrm{a}-\mathrm{butyl}-4,4,7-t r i m e t h y l d e c a h y d r o-7 \mathrm{aH}-c y c l o p e n t a[c d]$ inden-7a-ol [32]

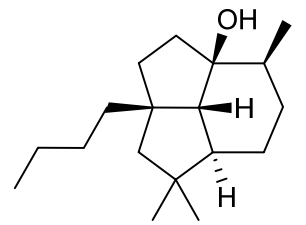

This compound was prepared on $0.252 \mathrm{mmol}$ scale (scale B). After $22 \mathrm{~d}$ a conversion of approximately $85 \%$ was reached. At this point the reaction mixture was directly loaded onto a column of silica (approximately $40 \mathrm{~g}$ ) and chromatographed, eluting first with pentane (to remove solvent and nonpolar by-products) then with pentane / diethyl ether 9:1 to give the title compound as a colorless oil.

Yield: $16.0 \mathrm{mg}, 24 \%$

$\mathbf{R}_{f} 0.38$ (pentane / $\mathrm{Et}_{2} \mathrm{O}, 9: 1$ )

IR $v_{\max }\left(\right.$ thin film) $/ \mathrm{cm}^{-1} 3475 \mathrm{br}, 2927 \mathrm{~s}, 2859 \mathrm{~s}, 1461 \mathrm{~m}, 1364 \mathrm{~m}, 1302 \mathrm{~m}, 1259 \mathrm{~m}, 1032 \mathrm{~m}, 990 \mathrm{~m}, 976 \mathrm{~m}$

${ }^{1} \mathbf{H}$ NMR $\left(500 \mathrm{MHz}, \mathrm{CDCl}_{3}\right) \delta 2.05-1.95(\mathrm{~m}, 1 \mathrm{H}, \mathrm{H} 3), 1.91(\mathrm{dt}, J=13.2,8.0 \mathrm{~Hz}, 1 \mathrm{H}, \mathrm{H} 2), 1.87-1.81$ (m, 1H, H9), $1.74-1.69$ (m, 1H, H2), $1.68-1.63$ (m, 2H, H5, H8), $1.63-1.55$ (m, 1H, H14), 1.55 1.45 (m, 3H, H3, 2x H11), $1.44-1.38$ (m, 1H, H10, H14), 1.35 (d, J=13.5 Hz, 1H, H5), $1.32-1.17$ (m, 6H, H7, H10, 2x H16, 2x H17), 0.96 (d, $J=7.1 \mathrm{~Hz}, 3 \mathrm{H}, 3 x \mathrm{H} 15), 0.94$ (s, 3H, 3x H12), $0.93-0.85$ (t, $6.9 \mathrm{~Hz}, 3 \mathrm{H}, 3 \mathrm{x} \mathrm{H1}), 0.80$ (s, 3H, 3x H13).

${ }^{13} \mathbf{C}$ NMR $\left(126 \mathrm{MHz}, \mathrm{CDCl}_{3}\right) \delta 84.8\left(\mathrm{C}_{\mathrm{q}}, \mathrm{C} 1\right), 62.9(\mathrm{CH}, \mathrm{C} 8), 55.4\left(\mathrm{CH}_{2}, \mathrm{C} 5\right), 51.1(\mathrm{CH}, \mathrm{C} 7), 50.2\left(\mathrm{C}_{\mathrm{q}}\right.$, C4), $43.1\left(\mathrm{CH}_{2}, \mathrm{C} 14\right), 40.3\left(\mathrm{C}_{\mathrm{q}}, \mathrm{C} 6\right), 39.24\left(\mathrm{CH}_{2}, \mathrm{C} 3\right), 39.20\left(\mathrm{CH}_{2}, \mathrm{C} 2\right), 36.7(\mathrm{CH}, \mathrm{C} 9), 29.7\left(\mathrm{CH}_{2}\right.$, C11), $29.0\left(\mathrm{CH}_{3}, \mathrm{C} 12\right), 28.4\left(\mathrm{CH}_{2}, \mathrm{C} 16\right), 23.7\left(\mathrm{CH}_{2}, \mathrm{C} 17\right), 22.6\left(\mathrm{CH}_{3}, \mathrm{C} 13\right), 20.1\left(\mathrm{CH}_{2}, \mathrm{C} 10\right), 15.6$ $\left(\mathrm{CH}_{3}, \mathrm{C} 15\right), 14.4\left(\mathrm{CH}_{3}, \mathrm{C} 18\right)$.

HRMS $\left(\mathrm{ESI}^{+}\right) \mathrm{m} / \mathrm{z}$ calculated for $\mathrm{C}_{18} \mathrm{H}_{32} \mathrm{NaO}\left[\mathrm{M}+\mathrm{Na}^{+}\right]: 287.2345$; found: 287.2341 .

(2aS $\left., 2 \mathrm{a}^{1} R, 4 \mathrm{a} R, 7 S, 7 \mathrm{a} S\right)$-2a-iso-butyl-4,4,7-trimethyldecahydro-7aH-cyclopenta[cd]inden-7a-ol [34] 


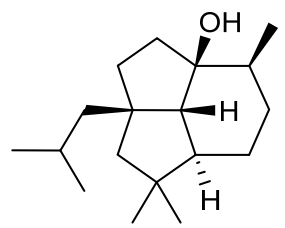

This compound was prepared on $0.252 \mathrm{mmol}$ scale (scale B). After $25 \mathrm{~d}$ a conversion of approximately $85 \%$ was reached. At this point the reaction mixture was directly loaded onto a column of silica (approximately $40 \mathrm{~g}$ ) and chromatographed, eluting first with pentane (to remove solvent and nonpolar by-products) then with pentane / diethyl ether 9:1 to give the title compound as a white solid.

Yield: $13.3 \mathrm{mg}, 20 \%$

$\mathbf{R}_{f} 0.37$ (pentane / $\mathrm{Et}_{2} \mathrm{O}, 9: 1$ )

IR $v_{\max }\left(\right.$ thin film) $/ \mathrm{cm}^{-1} 3467 \mathrm{br}, 2949 \mathrm{~s}, 2926 \mathrm{~s}, 2863 \mathrm{~s}, 1461 \mathrm{~m}, 1364 \mathrm{~m}, 1382 \mathrm{~m}, 1364 \mathrm{~m}, 1302 \mathrm{~m}, 1030 \mathrm{~m}$, 991m, $931 \mathrm{~m}$

${ }^{1} \mathbf{H}$ NMR $\left(500 \mathrm{MHz}, \mathrm{CDCl}_{3}\right) \delta 2.07-1.95(\mathrm{~m}, 2 \mathrm{H}, \mathrm{H} 2, \mathrm{H} 3), 1.89-1.81(\mathrm{~m}, 1 \mathrm{H}, \mathrm{H} 9), 1.81-1.75$ (m, 1H, H16), $1.75-1.68$ (m, 3H, H3, H5, H14), 1.68 - 1.62 (m, 1H, H8), $1.56-1.37$ (m, 4H, H2, H5, H10, H11), 1.33 (m, 1H, H14), 1.30 (d, J=6.9 Hz, 1H, OH), $1.28-1.17$ (m, 3H, H7, H10, H11), 0.95 (m, 6H, 3x H12, 3x H15), 0.92 (d, $J=3.8 \mathrm{~Hz}, 3 \mathrm{H}, 3 \mathrm{x} \mathrm{H17)}, 0.90$ (d, $J=3.8 \mathrm{~Hz}, 3 \mathrm{H}, 3 \times \mathrm{x} 18), 0.80$ (s, $3 \mathrm{H}, 3 \times \mathrm{H} 13)$.

${ }^{13} \mathrm{C}$ NMR $\left(126 \mathrm{MHz}, \mathrm{CDCl}_{3}\right) \delta 84.5\left(\mathrm{C}_{\mathrm{q}}, \mathrm{C} 1\right), 65.1(\mathrm{CH}, \mathrm{C} 8), 56.2\left(\mathrm{CH}_{2}, \mathrm{C} 5\right), 51.6\left(\mathrm{CH}_{2}, \mathrm{C} 14\right), 50.28$ $\left(\mathrm{C}_{\mathrm{q}}, \mathrm{C} 4\right), 50.26(\mathrm{CH}, \mathrm{C} 7), 40.6\left(\mathrm{C}_{\mathrm{q}}, \mathrm{C} 6\right), 39.7\left(\mathrm{CH}_{2}, \mathrm{C} 3\right), 39.2\left(\mathrm{CH}_{2}, \mathrm{C} 2\right), 36.6(\mathrm{CH}, \mathrm{C} 9), 29.4\left(\mathrm{CH}_{2}\right.$, C11), $29.0\left(\mathrm{CH}_{3}, \mathrm{C} 12\right), 26.6(\mathrm{CH}, \mathrm{C} 16), 24.9\left(\mathrm{CH}_{3}, \mathrm{C} 17\right), 24.5\left(\mathrm{CH}_{3}, \mathrm{C} 18\right), 22.7\left(\mathrm{CH}_{3}, \mathrm{C} 13\right), 20.0\left(\mathrm{CH}_{2}\right.$, $\mathrm{C} 10), 15.5\left(\mathrm{CH}_{3}, \mathrm{C} 15\right)$.

HRMS $\left(\mathrm{ESI}^{+}\right) \mathrm{m} / \mathrm{z}$ calculated for $\mathrm{C}_{18} \mathrm{H}_{32} \mathrm{NaO}\left[\mathrm{M}+\mathrm{Na}^{+}\right]: 287.2345$; found: 287.2345.

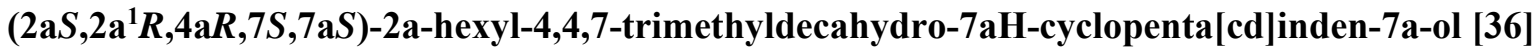

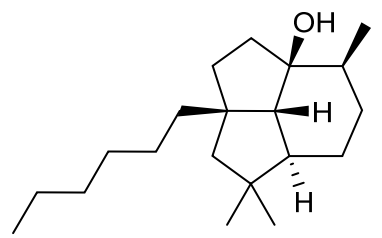

This compound was prepared on $0.252 \mathrm{mmol}$ scale (scale B). After $19 \mathrm{~d}$ a conversion of approximately $74 \%$ was reached. At this point the reaction mixture was directly loaded onto a column of silica (approximately $40 \mathrm{~g}$ ) and chromatographed, eluting first with pentane (to remove solvent and nonpolar by-products) then with pentane / diethyl ether 9:1 to give the title compound as a colorless oil.

Yield: $19.9 \mathrm{mg}, 27 \%$

$\mathbf{R}_{f} 0.32$ (pentane / $\mathrm{Et}_{2} \mathrm{O}, 9: 1$ )

IR $v_{\max }\left(\right.$ thin film) $/ \mathrm{cm}^{-1} 3474 \mathrm{br}, 2925 \mathrm{~s}, 2858 \mathrm{~s}, 1461 \mathrm{~m}, 1363 \mathrm{~m}, 1028 \mathrm{~m}, 994 \mathrm{~m}, 973 \mathrm{~m}, 929 \mathrm{~m}$

${ }^{1} \mathbf{H}$ NMR $\left(500 \mathrm{MHz}, \mathrm{CDCl}_{3}\right) \delta 2.00(\mathrm{dt}, J=13.2,8.3 \mathrm{~Hz}, 1 \mathrm{H}, \mathrm{H} 2), 1.91(\mathrm{dt}, J=13.2,8.0 \mathrm{~Hz}, 1 \mathrm{H}, \mathrm{H} 3)$, $1.88-1.79$ (m, 1H, H9), $1.74-1.68$ (m, 1H, H2), $1.67-1.62$ (m, 2H, H5, H8), 1.59 (td, J = 10.9, 3.4 Hz, 1H, H14), 1.55 - 1.45 (m, 2H, H3, H11), 1.45 - 1.37 (m, 3H, H10, H11, H14), 1.35 (d, J = 13.2 $\mathrm{Hz}, 1 \mathrm{H}, \mathrm{H} 5), 1.32$ - 1.20 (m, 9H, H7, 2x H16, 2x H17, 2x H18, 2x H19), 0.96 (d, J = 7.1 Hz, 3H, 3x H15), 0.94 (s, 3H, 3x H13), 0.87 (m, 3H, 3x H20), 0.80 (s, 3H, 3x H12). 
${ }^{13} \mathrm{C}$ NMR $\left(126 \mathrm{MHz}, \mathrm{CDCl}_{3}\right) \delta 84.8\left(\mathrm{C}_{\mathrm{q}}, \mathrm{C} 1\right), 62.9(\mathrm{CH}, \mathrm{C} 8), 55.4\left(\mathrm{CH}_{2}, \mathrm{C} 5\right), 51.1(\mathrm{CH}, \mathrm{C} 7), 50.3\left(\mathrm{C}_{\mathrm{q}}\right.$, C4), $43.4\left(\mathrm{CH}_{2}, \mathrm{C} 14\right), 40.3\left(\mathrm{C}_{\mathrm{q}}, \mathrm{C} 6\right), 39.23\left(\mathrm{CH}_{2}, \mathrm{C} 3\right), 39.20\left(\mathrm{CH}_{2}, \mathrm{C} 2\right), 36.7(\mathrm{CH}, \mathrm{C} 9), 32.1\left(\mathrm{CH}_{2}\right.$, $\mathrm{C} 18), 30.4\left(\mathrm{CH}_{2}, \mathrm{C} 17\right), 29.8\left(\mathrm{CH}_{2}, \mathrm{C} 11\right), 29.0\left(\mathrm{CH}_{3}, \mathrm{C} 13\right), 26.1\left(\mathrm{CH}_{2}, \mathrm{C} 16\right), 22.9\left(\mathrm{CH}_{2}, \mathrm{C} 19\right), 22.6$ $\left(\mathrm{CH}_{3}, \mathrm{C} 12\right), 20.1\left(\mathrm{CH}_{2}, \mathrm{C} 10\right), 15.6\left(\mathrm{CH}_{3}, \mathrm{C} 15\right), 14.3\left(\mathrm{CH}_{3}, \mathrm{C} 20\right)$.

HRMS $\left(\mathrm{ESI}^{+}\right) \mathrm{m} / \mathrm{z}$ calculated for $\mathrm{C}_{20} \mathrm{H}_{36} \mathrm{NaO}\left[\mathrm{M}+\mathrm{Na}^{+}\right]: 315.2658$; found: 315.2653

$\left(2 \mathrm{a} S, 2 \mathrm{a}^{1} R, 4 \mathrm{a} R, 7 S, 7 \mathrm{a} S\right)$-2a-octyl-4,4,7-trimethyldecahydro-7aH-cyclopenta[cd]inden-7a-ol [38]

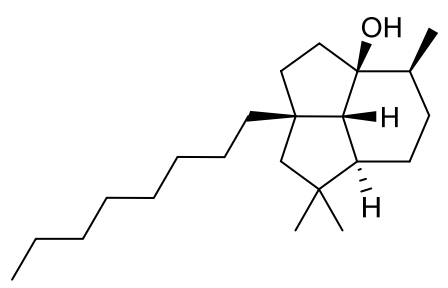

This compound was prepared on a $0.1 \mathrm{mmol}$ scale. The reaction mixture was directly loaded after $11 \mathrm{~d}$ onto a column of silica (approximately $30 \mathrm{~g}$ ) and chromatographed, eluting first with pentane (to remove solvent and nonpolar by-products) then with pentane / diethyl ether 9:1. Further purification of title compound was required using $\mathrm{AgNO}_{3}$-coated silica $\left(10 \% \mathrm{AgNO}_{3}\right)$ in order to separate it from unreacted substrate. The desired compound was obtained as colorless oil.

Yield: $2.7 \mathrm{mg}, 9 \%$

$\mathbf{R}_{f} 0.44$ (pentane / $\mathrm{Et}_{2} \mathrm{O}, 9: 1$ )

IR $v_{\max }\left(\right.$ thin film) $/ \mathrm{cm}^{-1} 3469 \mathrm{br}, 2924 \mathrm{~s}, 2856 \mathrm{~s}, 1461 \mathrm{w}, 1363 \mathrm{w}, 1301 \mathrm{w}, 1258 \mathrm{w}, 975 \mathrm{w}$

${ }^{1} \mathbf{H}$ NMR $\left(500 \mathrm{MHz}, \mathrm{CDCl}_{3}\right) \delta 2.00(\mathrm{ddd}, J=13.2,9.0,7.7 \mathrm{~Hz}, 1 \mathrm{H}, \mathrm{H} 2), 1.91$ (dt, $J=13.2,8.0 \mathrm{~Hz}, 1 \mathrm{H}$, H3), 1.84 (ddt, $J=11.8,7.3,2.3 \mathrm{~Hz}, 1 \mathrm{H}, \mathrm{H} 9$ ), 1.71 (dddd, $J=13.1,8.2,4.7,1.3 \mathrm{~Hz}, 1 \mathrm{H}, \mathrm{H} 1$ ), 1.67 1.62 (m, 2H, H5, H8), 1.62 - 1.45 (m, 3H, H3, H10, H14), 1.45 - 1.38 (m, 2H, H11, H14), 1.35 (d, J = $13.3 \mathrm{~Hz}, 1 \mathrm{H}, \mathrm{H} 5$ ), 1.33 - 1.20 (m, 15H, H7, H10, H11, 2x H16, 2x H17, 2x H18, 2x H19, 2x H20, 2x H21), 0.96 (d, $J=7.0 \mathrm{~Hz}, 3 \mathrm{H}, 3 \times \mathrm{H} 15), 0.95$ (s, 3H, 3x H13), 0.88 (t, $J=6.9 \mathrm{~Hz}, 3 \mathrm{H}, 3 \mathrm{x} \mathrm{H} 22), 0.80$ (s, $3 \mathrm{H}, 3 \mathrm{x} \mathrm{H} 12)$.

${ }^{13} \mathrm{C}$ NMR $\left(126 \mathrm{MHz}, \mathrm{CDCl}_{3}\right) \delta 84.8\left(\mathrm{C}_{\mathrm{q}}, \mathrm{C} 1\right), 62.9(\mathrm{CH}, \mathrm{C} 8), 55.5\left(\mathrm{CH}_{2}, \mathrm{C} 5\right), 51.2(\mathrm{CH}, \mathrm{C} 7), 50.3\left(\mathrm{C}_{\mathrm{q}}\right.$, $\mathrm{C} 4), 43.4\left(\mathrm{CH}_{2}, \mathrm{C} 14\right), 40.3\left(\mathrm{C}_{\mathrm{q}}, \mathrm{C} 6\right), 39.23\left(\mathrm{CH}_{2}, \mathrm{C} 3\right), 39.21\left(\mathrm{CH}_{2}, \mathrm{C} 2\right), 36.7(\mathrm{CH}, \mathrm{C} 9), 32.1\left(\mathrm{CH}_{2}\right.$, $\mathrm{C} 20), 30.7\left(\mathrm{CH}_{2}, \mathrm{C} 17\right), 29.9\left(\mathrm{CH}_{2}, \mathrm{C} 18\right), 29.7\left(\mathrm{CH}_{2}, \mathrm{C} 10\right), 29.5\left(\mathrm{CH}_{2}, \mathrm{C} 19\right), 29.0\left(\mathrm{CH}_{3}, \mathrm{C} 13\right), 26.2$ $\left(\mathrm{CH}_{2}, \mathrm{C} 16\right), 22.8\left(\mathrm{CH}_{2}, \mathrm{C} 21\right), 22.6\left(\mathrm{CH}_{3}, \mathrm{C} 12\right), 20.1\left(\mathrm{CH}_{2}, \mathrm{C} 11\right), 15.6\left(\mathrm{CH}_{3}, \mathrm{C} 15\right), 14.3\left(\mathrm{CH}_{3}, \mathrm{C} 22\right)$.

HRMS (ESI ${ }^{+}$) $m / z$ calculated for $\mathrm{C}_{22} \mathrm{H}_{40} \mathrm{NaO}\left[\mathrm{M}+\mathrm{Na}^{+}\right]$: 343.2971 ; found: 343.2966

\section{Note on the workup method used for large-scale reaction mixtures}

Due to concerns that the concentration of the reaction mixture during the solvent evaporation step might accelerate the rearrangement of presilphiperfolan-1 $\beta$-ol to rearranged alkene $\mathbf{2 6}$, in the optimized procedure for the preparation of compound (-)-4, the solvent was not removed and the reaction mixture was loaded directly onto a column of silica. This was found to not interfere with the purification process. Same was applied for the purification of other unnatural derivatives of (-)-4. 


\section{Minor products of the cyclisation reaction}

$\left(2 \mathrm{a} S, 2 \mathrm{a}^{1} R, 5 S, 7 \mathrm{a} R\right)-1,1,2 \mathrm{a}, 5$-tetramethyl-2,2a,2a1,3,5,6,7,7a-octahydro-1H-cyclopenta[cd]indene (10)

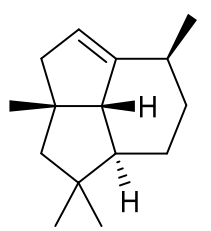

This compound $\mathbf{1 0}$ was isolated as a side product from a large scale reaction $(1.8 \mathrm{mmol})$ using substrate 23. $\mathrm{AgNO}_{3}$-coated silica $\left(10 \%\right.$ of $\left.\mathrm{AgNO}_{3}\right)$ was used for further purification.

The ${ }^{1} \mathrm{H}$ NMR spectroscopic data is consistent with that reported in the literature. ${ }^{6}$

Yield: $27 \mathrm{mg}, 8 \%$

Rf 0.74 (pentane)

${ }^{1} \mathbf{H}$ NMR $\left(500 \mathrm{MHz}, \mathrm{CDCl}_{3}\right) \delta 4.82(\mathrm{~s}, 1 \mathrm{H}), 2.76-2.69(\mathrm{~m}, 1 \mathrm{H}), 2.29(\mathrm{~s}, 2 \mathrm{H}), 2.18(\mathrm{~d}, J=11.6 \mathrm{~Hz}$, $1 \mathrm{H}), 1.71-1.67(\mathrm{~m}, 2 \mathrm{H}), 1.63-1.47(\mathrm{~m}, 4 \mathrm{H}), 1.37-1.23(\mathrm{~m}, 1 \mathrm{H}), 1.08(\mathrm{~s}, 3 \mathrm{H}), 1.08(\mathrm{~d}, J=12.0 \mathrm{~Hz}$, $3 \mathrm{H}), 0.93(\mathrm{~s}, 3 \mathrm{H}), 0.87(\mathrm{~s}, 3 \mathrm{H})$

${ }^{13} \mathrm{C}$ NMR $\left(125 \mathrm{MHz}, \mathrm{CDCl}_{3}\right) \delta 151.7,113.0,63.2,62.8,59.5,51.6,43.2,42.4,36.6,32.5,30.2,27.9$, $22.0,21.4,18.9$

$\left(2 \mathrm{a} S, 2 \mathrm{a}^{1} S, 4 \mathrm{a} S, 7 S, 7 \mathrm{a} S\right)-2 \mathrm{a}, 4,4 \mathrm{a}, 7-t e t r a m e t h y l-2,2 \mathrm{a}, 2 \mathrm{a} 1,4 \mathrm{a}, 5,6,7,7 \mathrm{a}-\mathrm{octahydro}-1 \mathrm{H}-$ cyclopenta[cd]indene (26)

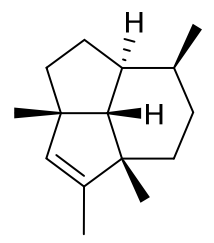

In a pressure tube, resorcinarene $1(600 \mathrm{mg}, 0.09 \mathrm{mmol}, 0.10$ eq. of capsule I) was dissolved in chloroform $(10 \mathrm{~mL})$ by gentle heating. Substrate $23(200 \mathrm{mg}, 0.90 \mathrm{mmol}, 1.0 \mathrm{eq})$ was added as a solution in chloroform $(4 \mathrm{~mL}$, rinsing with $2 \times 3 \mathrm{~mL})$. Further chloroform was added to adjust the reaction volume to $26.5 \mathrm{~mL}$, then $\mathrm{HCl}$ stock solution in chloroform was added $(392 \mu \mathrm{L}, 27.0 \mu \mathrm{mol}, 0.03 \mathrm{eq})$ and the reaction mixture was stirred at $30{ }^{\circ} \mathrm{C}$ (internal temperature). Reaction progress was monitored by gas chromatography as previously described. After $12 \mathrm{~d}$ a conversion of approximately $90 \%$ was reached (internal standard was not added in this reaction to facilitate isolation of products). The solvent was partially (ca. $75 \%$ ) removed by heating $\left(\right.$ at $\left.40{ }^{\circ} \mathrm{C}\right)$ on a rotary evaporator under reduced pressure $(700$ 750 mbar). The mixture was then passed through a column of silica (approximately $100 \mathrm{~g}$, using pentane as the eluent) to partially remove resorcinarene 1 and to separate nonpolar products (elimination product 10, alkene 26 and caryophyllene 15) from polar ones (presilphiperfolan-1 $\beta$-ol (-)-4 and unreacted substrate 23). The combined former fractions were re-subjected to flash chromatography (approximately $50 \mathrm{~g}$ of silica, pentane as the eluent) to provide a fraction of alkene $\mathbf{2 6}$ contaminated by elimination product 10 (colorless oil, 3.6:1 of 26:10). It was further purified by using $\mathrm{AgNO}_{3}$-coated silica $\left(10 \% \mathrm{AgNO}_{3}\right)$ to provide $\mathbf{2 6}$ in $90 \%$ GC purity.

Yield: $48 \mathrm{mg}, 24 \%$ 
$\mathbf{R}_{f} 0.86$ (pentane)

IR $v_{\max }($ thin film $) / \mathrm{cm}^{-1} 2923 \mathrm{~s}, 2862 \mathrm{~s}, 1455 \mathrm{~m}, 1370 \mathrm{~m}, 839 \mathrm{~m}$

${ }^{1} \mathbf{H}$ NMR $\left(500 \mathrm{MHz}, \mathrm{CDCl}_{3}\right) \delta 5.09(\mathrm{~s}, 1 \mathrm{H}), 1.89-1.81(\mathrm{~m}, 1 \mathrm{H}), 1.80-1.66(\mathrm{~m}, 3 \mathrm{H}), 1.59(\mathrm{dd}, J=$ $12.3,7.0 \mathrm{~Hz}, 1 \mathrm{H}), 1.56(\mathrm{~d}, J=1.5 \mathrm{~Hz}, 3 \mathrm{H}), 1.52-1.40(\mathrm{~m}, 2 \mathrm{H}), 1.33-1.26(\mathrm{~m}, 1 \mathrm{H}), 1.28-1.24(\mathrm{~m}$, 2H), $1.08(\mathrm{~s}, 3 \mathrm{H}), 1.02(\mathrm{~s}, 3 \mathrm{H}), 0.91-0.87(\mathrm{~m}, 1 \mathrm{H}), 0.83(\mathrm{~d}, J=6.7 \mathrm{~Hz}, 3 \mathrm{H})$

${ }^{13} \mathbf{C}$ NMR $\left(125 \mathrm{MHz}, \mathrm{CDCl}_{3}\right) \delta 143.0,134.1,58.0,51.0,48.6,43.7,40.1,31.4,29.9,28.8,28.1,27.6$, $24.9,15.7,12.0$

HRMS $\left(\mathrm{ESI}^{+}\right) \mathrm{m} / \mathrm{z}$ calculated for $\mathrm{C}_{15} \mathrm{H}_{25}\left[\mathrm{M}+\mathrm{H}^{+}\right]: 205.1951$; found 205.1948

\section{Derivatization of alkene 26}

Allylic oxidation of alkene $\mathbf{2 6}$ was carried out in order to obtain a more polar derivative that could be more easily purified, as well as to allow for the preparation of a crystalline derivative.

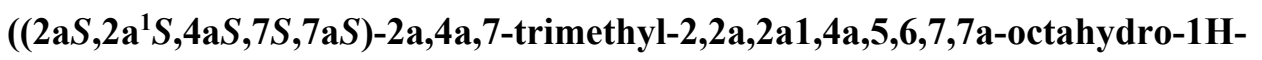
cyclopenta[cd]inden-4-yl)methanol (27)

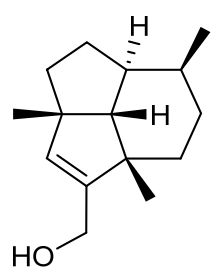

In a pressure tube, alkene 26 (28 $\mathrm{mg}, 0.137 \mathrm{mmol}, 1.0$ eq., 3.6:1 of 26:10) was dissolved in DCM (4 $\mathrm{mL}$ ). $\mathrm{SeO}_{2}\left(30.4 \mathrm{mg}, 0.274 \mathrm{mmol}, 2.0 \mathrm{eq}\right.$ ) was added and the mixture was stirred at $39{ }^{\circ} \mathrm{C}$ (oil bath temperature) for $36 \mathrm{~h}$. Control by TLC at this time showed the reaction to be incomplete; another portion of $\mathrm{SeO}_{2}$ was added (30.4 mg, $0.274 \mathrm{mmol}, 2.0 \mathrm{eq}$ ) and the reaction stirred for a further $18 \mathrm{~h}$ at the same temperature. The reaction mixture was then concentrated in vacuo and directly subjected to flash chromatography (pentane / $\mathrm{Et}_{2} \mathrm{O}, 9: 1$ ) to give the title compound (yellow oil, $12.0 \mathrm{mg}, 40 \%$ ) as well as aldehyde S-1.

Yield: $9.0 \mathrm{mg}, 30 \%$

Optical rotation $[\alpha]_{\mathrm{D}}^{25.0}+92.5\left(\mathrm{c} 0.020, \mathrm{CHCl}_{3}\right)$

$\mathbf{R}_{f} 0.08$ (pentane / diethyl ether, 9:1)

IR $v_{\max }\left(\right.$ thin film) $/ \mathrm{cm}^{-1} 3319 \mathrm{br}, 2942 \mathrm{~s}, 2927 \mathrm{~s}, 2863 \mathrm{~s}, 1455 \mathrm{~m}, 1380 \mathrm{~m}, 1371 \mathrm{~m}, 1099 \mathrm{~m}, 1016 \mathrm{~m}, 849 \mathrm{~m}$

${ }^{1} \mathbf{H}$ NMR $\left(500 \mathrm{MHz}, \mathrm{CDCl}_{3}\right) \delta 5.42(\mathrm{~s}, 1 \mathrm{H}), 4.19-4.11(\mathrm{~m}, 2 \mathrm{H}), 1.92-1.82(\mathrm{~m}, 1 \mathrm{H}), 1.80-1.70(\mathrm{~m}$, $2 \mathrm{H}), 1.67-1.59(\mathrm{~m}, 1 \mathrm{H}), 1.56-1.43(\mathrm{~m}, 3 \mathrm{H}), 1.35(\mathrm{~d}, J=12.5 \mathrm{~Hz}, 1 \mathrm{H}), 1.32-1.21(\mathrm{~m}, 1 \mathrm{H}), 1.18(\mathrm{dt}$, $J=13.1,3.5 \mathrm{~Hz}, 1 \mathrm{H}), 1.13(\mathrm{~s}, 3 \mathrm{H}), 1.11(\mathrm{~s}, 3 \mathrm{H}), 1.04-0.94(\mathrm{~m}, 1 \mathrm{H}), 0.83(\mathrm{~d}, J=6.8 \mathrm{~Hz}, 3 \mathrm{H})$.

${ }^{13} \mathbf{C}$ NMR $\left(125 \mathrm{MHz}, \mathrm{CDCl}_{3}\right) \delta 147.1,134.7,60.0,58.7,51.2,48.2,43.9,40.1,31.8,30.0,28.7,28.1$, $27.7,26.1,15.8$

HRMS $\left(\mathrm{ESI}^{+}\right) \mathrm{m} / \mathrm{z}$ calculated for $\mathrm{C}_{15} \mathrm{H}_{24} \mathrm{NaO}\left[\mathrm{M}+\mathrm{Na}^{+}\right]: 243.1719$; found 243.1723 


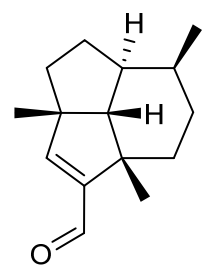

Alcohol 27 (12.0 mg, $0.055 \mathrm{mmol}, 1.0$ eq) was dissolved in DCM (500 $\mu \mathrm{L}), \mathrm{MnO}_{2}(19.0 \mathrm{mg}, 0.218$ mmol, 4.0 eq) was added and the mixture was stirred at $\mathrm{rt}$ for $18 \mathrm{~h}$. At this point control by TLC showed that the reaction was incomplete; another portion of $\mathrm{MnO}_{2}(9.5 \mathrm{mg}, 0.109 \mathrm{mmol}, 2.0 \mathrm{eq})$ was added and the stirring continued for a further $2 \mathrm{~h}$. The reaction mixture was then filtered through celite, eluting with DCM. Removal of the solvent in vacuo gave the title compound (yellow oil), which was of sufficient purity to be used in the next step with no further purification.

Yield: $9.2 \mathrm{mg}, 77 \%$

$\mathbf{R}_{f} 0.48$ (pentane / diethyl ether, 9:1)

IR $v_{\max }\left(\right.$ thin film) $/ \mathrm{cm}^{-1} 2949 \mathrm{~m}, 2863 \mathrm{w}, 1639 \mathrm{~s}, 1304 \mathrm{~s}, 1075 \mathrm{~s}, 890 \mathrm{~m}, 823 \mathrm{~m}$

${ }^{1} \mathbf{H}$ NMR $\left(500 \mathrm{MHz}, \mathrm{CDCl}_{3}\right) \delta 9.72(\mathrm{~s}, 1 \mathrm{H}), 6.55(\mathrm{~s}, 1 \mathrm{H}), 1.92-1.85(\mathrm{~m}, 1 \mathrm{H}), 1.80-1.72(\mathrm{~m}, 2 \mathrm{H})$, $1.70-1.62(\mathrm{~m}, 3 \mathrm{H}), 1.54-1.46(\mathrm{~m}, 2 \mathrm{H}), 1.42(\mathrm{~d}, J=12.6 \mathrm{~Hz}, 1 \mathrm{H}), 1.37-1.27(\mathrm{~m}, 1 \mathrm{H}), 1.22(\mathrm{~s}, 3 \mathrm{H})$, $1.20(\mathrm{~s}, 3 \mathrm{H}), 1.02-0.94(\mathrm{~m}, 1 \mathrm{H}), 0.83(\mathrm{~d}, J=6.9 \mathrm{~Hz}, 3 \mathrm{H})$.

${ }^{13} \mathrm{C}$ NMR $\left(125 \mathrm{MHz}, \mathrm{CDCl}_{3}\right) \delta 191.6,162.0,150.2,58.8,52.8,46.9,44.3,39.5,32.1,29.7,27.9,27.6$, $27.4,25.2,15.7$

HRMS $\left(\mathrm{ESI}^{+}\right) \mathrm{m} / \mathrm{z}$ calculated for $\mathrm{C}_{16} \mathrm{H}_{25} \mathrm{O}\left[\mathrm{M}+\mathrm{MeOH}-\mathrm{OH}^{-}\right]: 233.1900$; found 233.1901

(E)-2-(((2aS,2a1S,4aS,7S,7aS)-2a,4a,7-trimethyl-2,2a,2a1,4a,5,6,7,7a-octahydro-1Hcyclopenta[cd]inden-4-yl)methylene)hydrazine-1-carboxamide (28)

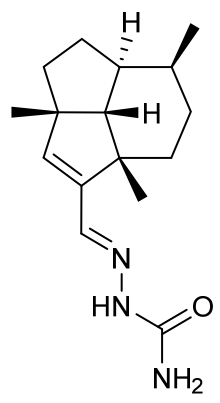

To a solution of semicarbazide hydrochloride ( $7.2 \mathrm{mg}, 0.064 \mathrm{mmol}, 2.0 \mathrm{eq})$ in water $(250 \mu \mathrm{L})$ was added sodium acetate $(5.3 \mathrm{mg}, 0.064 \mathrm{mmol}, 2.0 \mathrm{eq})$. After stirring at $\mathrm{rt}$ for $30 \mathrm{~min}$, to this solution was added a solution of aldehyde S-1 (7.0 mg, $0.032 \mathrm{mmol}, 1.0 \mathrm{eq})$ in EtOH $(100 \mu \mathrm{L}$, rinsing with $2 \times 100 \mu \mathrm{L})$. A precipitate was formed upon addition. The mixture was stirred at $50{ }^{\circ} \mathrm{C}$ for $2 \mathrm{~h}$, the solvent was then removed in vacuo and the crude product subjected to flash chromatography (DCM / MeOH, 49:1) to give the title compound as an orange solid.

Yield: $7.4 \mathrm{mg}, 84 \%$

$\mathbf{R}_{f} 0.35(\mathrm{DCM} / \mathrm{MeOH}, 49: 1)$ 
IR $v_{\max }\left(\right.$ thin film) $/ \mathrm{cm}^{-1} 3476 \mathrm{~m}, 3191 \mathrm{br}, 2944 \mathrm{~s}, 2930 \mathrm{~s}, 2865 \mathrm{~m}, 1691 \mathrm{~s}, 1580 \mathrm{~s}, 1445 \mathrm{~m}, 1142 \mathrm{~m}, 1099 \mathrm{~m}$

${ }^{1} \mathbf{H}$ NMR $\left(600 \mathrm{MHz}, \mathrm{CDCl}_{3}\right) \delta 8.83-8.28(\mathrm{~m}, 1 \mathrm{H}), 7.41(\mathrm{~s}, 1 \mathrm{H}), 5.87(\mathrm{br} \mathrm{s}, 1 \mathrm{H}), 5.82(\mathrm{~s}, 1 \mathrm{H}), 4.94(\mathrm{br}$ $\mathrm{s}, 1 \mathrm{H}), 1.94-1.85(\mathrm{~m}, 1 \mathrm{H}), 1.81-1.69(\mathrm{~m}, 2 \mathrm{H}), 1.69-1.52(\mathrm{~m}, 4 \mathrm{H}), 1.51-1.45(\mathrm{~m}, 1 \mathrm{H}), 1.40(\mathrm{~d}, J=$ $12.6 \mathrm{~Hz}, 1 \mathrm{H}), 1.36-1.28(\mathrm{~m}, 1 \mathrm{H}), 1.25(\mathrm{~s}, 3 \mathrm{H}), 1.15(\mathrm{~s}, 3 \mathrm{H}), 1.05-0.95(\mathrm{~m}, 1 \mathrm{H}), 0.83(\mathrm{~d}, J=6.8 \mathrm{~Hz}$, $3 \mathrm{H})$.

${ }^{13}$ C NMR $\left(150 \mathrm{MHz}, \mathrm{CDCl}_{3}\right) \delta 148.3,143.5,140.4,58.9,52.1,48.2,43.9,39.9,33.3,29.8,2 \times 28.0$, 27.7, 25.6, 15.9 (1 not seen)

HRMS $\left(\mathrm{ESI}^{+}\right) \mathrm{m} / \mathrm{z}$ calculated for $\mathrm{C}_{16} \mathrm{H}_{25} \mathrm{~N}_{3} \mathrm{NaO}\left[\mathrm{M}+\mathrm{Na}^{+}\right]: 298.1890$; found 298.1888 


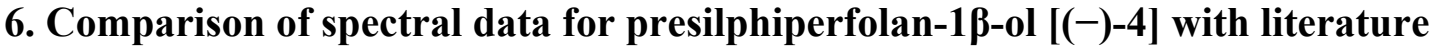

${ }^{1} \mathrm{H}$ Signals ${ }^{*}$

\begin{tabular}{|c|c|c|c|c|c|c|}
\hline Assignment & $\begin{array}{c}\text { Synthetic } \\
(-)-4, \text { this } \\
\text { work } \\
(\mathrm{ppm})\end{array}$ & $\begin{array}{c}\text { Multiplicity, } \\
J(\mathrm{~Hz})\end{array}$ & $\begin{array}{c}\text { Synthetic } \\
(-)-4, \\
\text { Stoltz } \\
(\mathrm{ppm})\end{array}$ & $\begin{array}{c}\text { Multiplicity, } \\
J(\mathrm{~Hz})\end{array}$ & $\begin{array}{c}\text { Natural } \\
(-)-4^{8} \\
(\mathrm{ppm})\end{array}$ & $\begin{array}{c}\text { Multiplicity, } \\
J(\mathrm{~Hz})\end{array}$ \\
\hline $\mathrm{C} 1$ & - & - & - & - & - & - \\
\hline $\mathrm{C} 2$ & $1.88-1.79$ & $\mathrm{~m}$ & $1.87-1.78$ & $\mathrm{~m}$ & $1.87-1.79$ & $\mathrm{~m}$ \\
\hline & $1.72-1.65$ & $\mathrm{~m}$ & $1.73-1.64$ & $\mathrm{~m}$ & $1.72-1.61$ & $\mathrm{~m}$ \\
\hline $\mathrm{C} 3$ & $1.88-1.79$ & $\mathrm{~m}$ & $1.89-1.81$ & $\mathrm{~m}$ & $1.87-1.79$ & $\mathrm{~m}$ \\
\hline & $1.64-1.55$ & $\mathrm{~m}$ & $1.63-1.55$ & $\mathrm{~m}$ & $1.61-1.54$ & $\mathrm{~m}$ \\
\hline $\mathrm{C} 4$ & - & - & - & - & - & - \\
\hline $\mathrm{C} 5$ & 1.50 & $\mathrm{~s}$ & 1.50 & $\mathrm{~s}$ & 1.50 & $\mathrm{~s}$ \\
\hline $\mathrm{C} 6$ & - & - & - & - & - & - \\
\hline $\mathrm{C} 7$ & $1.18-1.07$ & $\mathrm{~m}$ & $1.15-1.05$ & $\mathrm{~m}$ & $1.16-1.09$ & $\mathrm{~m}$ \\
\hline $\mathrm{C} 8$ & 1.56 & $\mathrm{~d}, 12.2$ & 1.55 & $\mathrm{br}, 12.3$ & $1.61-1.54$ & $\mathrm{~m}$ \\
\hline $\mathrm{C} 9$ & $1.64-1.55$ & $\mathrm{~m}$ & $1.63-1.55$ & $\mathrm{~m}$ & $1.61-1.54$ & $\mathrm{~m}$ \\
\hline $\mathrm{C} 10$ & $1.48-1.40$ & $\mathrm{~m}$ & $1.48-1.38$ & $\mathrm{~m}$ & $1.45-1.39$ & $\mathrm{~m}$ \\
\hline & $1.36-1.26$ & $\mathrm{~m}$ & $1.36-1.27$ & $\mathrm{~m}$ & $1.34-1.24$ & $\mathrm{~m}$ \\
\hline $\mathrm{C} 11$ & $1.36-1.26$ & $\mathrm{~m}$ & $1.33-1.25$ & $\mathrm{~m}$ & $1.34-1.24$ & $\mathrm{~m}$ \\
\hline & $1.18-1.07$ & $\mathrm{~m}$ & $1.19-1.09$ & $\mathrm{~m}$ & $1.16-1.09$ & $\mathrm{~m}$ \\
\hline $\mathrm{C} 12$ & 0.80 & $\mathrm{~s}$ & 0.80 & $\mathrm{~s}$ & 0.80 & $\mathrm{~s}$ \\
\hline $\mathrm{C} 13$ & 0.94 & $\mathrm{~s}$ & 0.94 & $\mathrm{~s}$ & 0.93 & $\mathrm{~s}$ \\
\hline $\mathrm{C} 14$ & 1.32 & $\mathrm{~s}$ & 1.33 & $\mathrm{~s}$ & 1.32 & $\mathrm{~s}$ \\
\hline $\mathrm{C} 15$ & 0.90 & $\mathrm{~d}, 7.0$ & 0.90 & $\mathrm{~d}, 7.0$ & 0.89 & $\mathrm{~d}, 7$ \\
\hline
\end{tabular}

${ }^{13}$ C Signals ${ }^{*}$

\begin{tabular}{|c|c|c|c|}
\hline Assignment & $\begin{array}{c}\text { Synthetic } \\
(-)-4, \text { this } \\
\text { work } \\
\text { (ppm) }\end{array}$ & $\begin{array}{c}\text { Synthetic } \\
(-\mathbf{-}) \\
\text { Stoltz }^{7} \\
(\mathrm{ppm})\end{array}$ & $\begin{array}{c}\text { Natural (-)- } \\
\mathbf{4}^{8}(\mathrm{ppm})\end{array}$ \\
\hline C1 & 84.5 & 84.5 & 84.3 \\
\hline C2 & 39.7 & 39.7 & 39.5 \\
\hline C3 & 42.7 & 42.7 & 42.4 \\
\hline C4 & 46.2 & 46.2 & 46.0 \\
\hline C5 & 59.4 & 59.4 & 59.2 \\
\hline C6 & 40.5 & 40.5 & 40.2 \\
\hline C7 & 51.7 & 51.7 & 51.5 \\
\hline C8 & 63.9 & 63.9 & 63.7 \\
\hline C9 & 36.9 & 36.9 & 36.7 \\
\hline C10 & 29.9 & 29.9 & 29.7 \\
\hline C11 & 20.3 & 20.3 & 20.1 \\
\hline C12 & 22.3 & 22.3 & 22.1 \\
\hline C13 & 28.8 & 28.8 & 28.5 \\
\hline C14 & 31.4 & 31.4 & 31.1 \\
\hline C15 & 15.7 & 15.8 & 15.5 \\
\hline
\end{tabular}

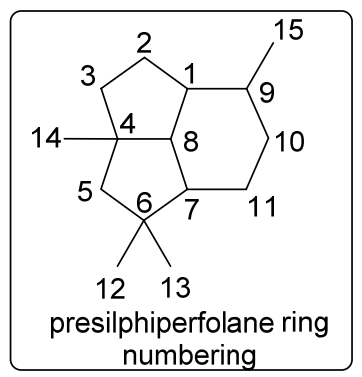

*All spectra recorded in $\mathrm{C}_{6} \mathrm{D}_{6}$ 


\section{Comparison of ${ }^{1} \mathrm{H}$ spectrum of alkene 10 with literature}

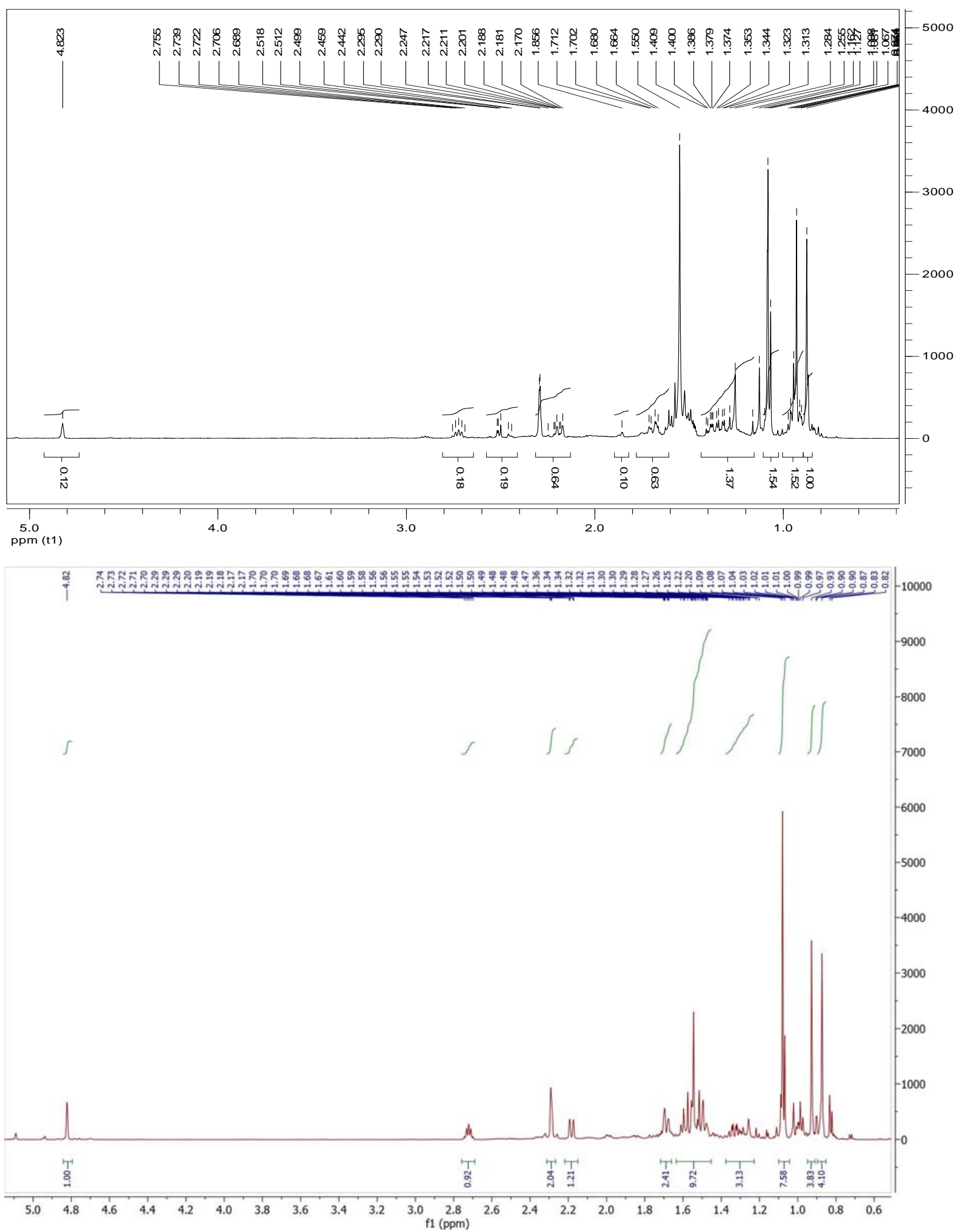

Top: ${ }^{1} \mathrm{H}$ spectrum of compound $\mathbf{1 0}$ taken from ref [8]. Bottom: ${ }^{1} \mathrm{H}$ spectrum of compound $\mathbf{1 0}$ recorded in this study. 


\section{References}

[1] L. Catti, A. Pöthig, K. Tiefenbacher, Adv. Synth. Catal. 2017, 359, 1331-1338.

[2] J. M. Köster, K. Tiefenbacher, ChemCatChem 2018, 10, 2941-2944.

[3] S. Shankar, R. M. Coates, J. Org. Chem. 1998, 63, 9177-9182.

[4] Q. Zhang, K. Tiefenbacher, Nat. Chem. 2015, 7, 197-202.

[5] a) K. Hayasaka, T. Ohtsuka, H. Shirahama, T. Matsumoto, Tetrahedron Lett. 1985, 26, 873-876; b) I. G. Collado, J. Aleu, A. J. Macías-Sánchez, R. Hernández-Galán, J. Nat. Prod. 1994, 57, 738746; c) L. Fitjer, A. Malich, C. Paschke, S. Kluge, R. Gerke, B. Rissom, J. Weiser, M. Noltemeyer, J. Am. Chem. Soc. 1995, 117, 9180-9189; d) K. Hayano, Y. Ohfune, H. Shirahama, T. Matsumoto, Chem. Lett. 1978, 7, 1301-1304; e) E. J. Corey, S. Nozoe, J. Am. Chem. Soc. 1964, 86, 1652-1653.

[6] S. C. Pinto, G. G. Leitão, H. R. Bizzo, N. Martinez, E. Dellacassa, F. M. dos Santos, F. L. P. Costa, M. B. d. Amorim, S. G. Leitão, Tetrahedron Lett. 2009, 50, 4785-4787.

[7] A. Y. Hong, B. M. Stoltz, Angew. Chem. Int. Ed. 2012, 51, 9674-9678.

[8] S. Melching, W. A. König, Phytochemistry 1999, 51, 517-523.

[9] Y. Zhao, D. G. Truhlar, Theor. Chem. Acc. 2008, 120, 215-241.

[10] a) W. J. Hehre, R. Ditchfield, J. A. Pople, J. Chem. Phys. 1972, 56, 2257-2261; b) G. A. Petersson, A. Bennett, T. G. Tensfeldt, M. A. Al-Laham, W. A. Shirley, J. Mantzaris, J. Chem. Phys. 1988, 89, 2193-2218; c) G. A. Petersson, M. A. Al-Laham, J. Chem. Phys. 1991, 94, 6081-6090.

[11] M. J. Frisch, G. W. Trucks, H. B. Schlegel, G. E. Scuseria, M. A. Robb, J. R. Cheeseman, G. Scalmani, V. Barone, G. A. Petersson, H. Nakatsuji, X. Li, M. Caricato, A. V. Marenich, J. Bloino, B. G. Janesko, R. Gomperts, B. Mennucci, H. P. Hratchian, J. V. Ortiz, A. F. Izmaylov, J. L. Sonnenberg, Williams, F. Ding, F. Lipparini, F. Egidi, J. Goings, B. Peng, A. Petrone, T. Henderson, D. Ranasinghe, V. G. Zakrzewski, J. Gao, N. Rega, G. Zheng, W. Liang, M. Hada, M. Ehara, K. Toyota, R. Fukuda, J. Hasegawa, M. Ishida, T. Nakajima, Y. Honda, O. Kitao, H. Nakai, T. Vreven, K. Throssell, J. A. Montgomery Jr., J. E. Peralta, F. Ogliaro, M. J. Bearpark, J. J. Heyd, E. N. Brothers, K. N. Kudin, V. N. Staroverov, T. A. Keith, R. Kobayashi, J. Normand, K. Raghavachari, A. P. Rendell, J. C. Burant, S. S. Iyengar, J. Tomasi, M. Cossi, J. M. Millam, M. Klene, C. Adamo, R. Cammi, J. W. Ochterski, R. L. Martin, K. Morokuma, O. Farkas, J. B. Foresman, D. J. Fox, Gaussian 16 Rev. A.03, Wallingford, CT, 2016. 


\section{Appendix A: Computational studies}

\section{Methods}

We employed the M06-2X hybrid DFT functional, ${ }^{9}$ with two basis sets, 6-31+G(d,p) and 6$311++\mathrm{G}(2 \mathrm{~d}, \mathrm{p}) .{ }^{10}$ All structures were geometry optimized using M06-2X with both basis sets, and stationary points were characterized via frequency calculations. Intrinsic reaction coordinate computations were performed for the transition states to verify that the transition states connect between the reactant and product states. All calculations employed the Gaussian 16 program, revision A.03. ${ }^{11}$

\section{Summary of results}

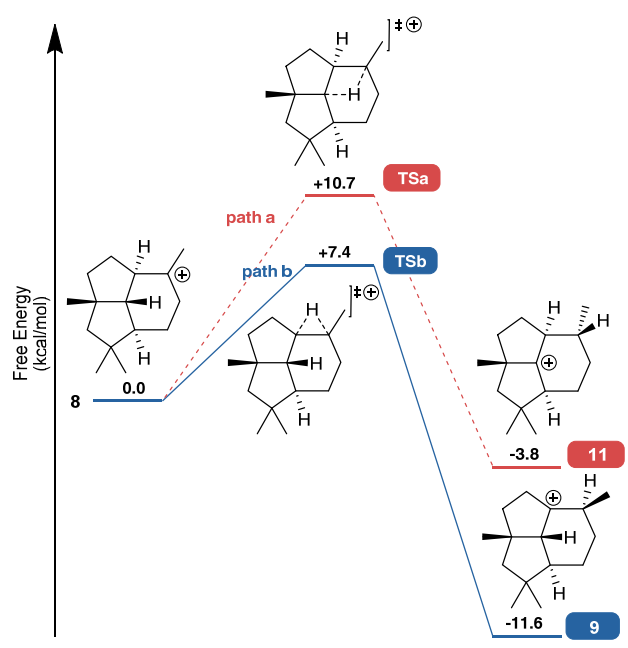

Table S-1. Relative free energies $(\mathrm{kcal} / \mathrm{mol})$ for formation of cations A and B from I.

\begin{tabular}{|l|c|c|c|c|}
\hline \multirow{2}{*}{ path $B$} & basis set & \multicolumn{3}{|c|}{ Relative free energy $(\mathrm{kcal} / \mathrm{mole})$} \\
\cline { 2 - 5 } & & $\mathbf{8}$ & TSb & $\mathbf{9}$ \\
\cline { 2 - 5 } & $6-31+g(d, p)$ & 0.00 & 7.42 & -11.46 \\
\cline { 2 - 5 } & $6-311++G(2 d, p)$ & 0.00 & 7.38 & -11.64 \\
\hline
\end{tabular}

\begin{tabular}{|l|c|c|c|c|}
\hline \multirow{3}{*}{ path $A$} & basis set & \multicolumn{3}{|c|}{ Relative free energy $(\mathrm{kcal} / \mathrm{mole})$} \\
\cline { 2 - 5 } & & $\mathbf{8}$ & TSa & $\mathbf{1 1}$ \\
\cline { 2 - 5 } & $6-31+g(d, p)$ & 0.00 & 9.96 & -3.92 \\
\cline { 2 - 5 } & $6-311++G(2 d, p)$ & 0.00 & 10.67 & -3.80 \\
\hline
\end{tabular}


Table S-2. Relative free energies $(\mathrm{kcal} / \mathrm{mol})$ for formation of (-)-presilphiperfolan-1 $\beta$-ol and (-)presilphiperfolan-8 $\alpha$-ol.

\begin{tabular}{|c|c|c|}
\hline \multirow{2}{*}{ basis set } & \multicolumn{2}{|c|}{ Relative free energy (kcal/mole) } \\
\cline { 2 - 3 } & $\begin{array}{c}\text { Path B } \\
\text { (-)-presilphiperfolan-16-ol }\end{array}$ & $\begin{array}{c}\text { Path A } \\
\text { (-)-presilphiperfolan-8 } \alpha \text {-ol }\end{array}$ \\
\hline $6-31+g(d, p)$ & 0.00 & 12.42 \\
\hline $6-311++G(2 d, p)$ & 0.00 & 12.58 \\
\hline
\end{tabular}

\section{Coordinates}

8

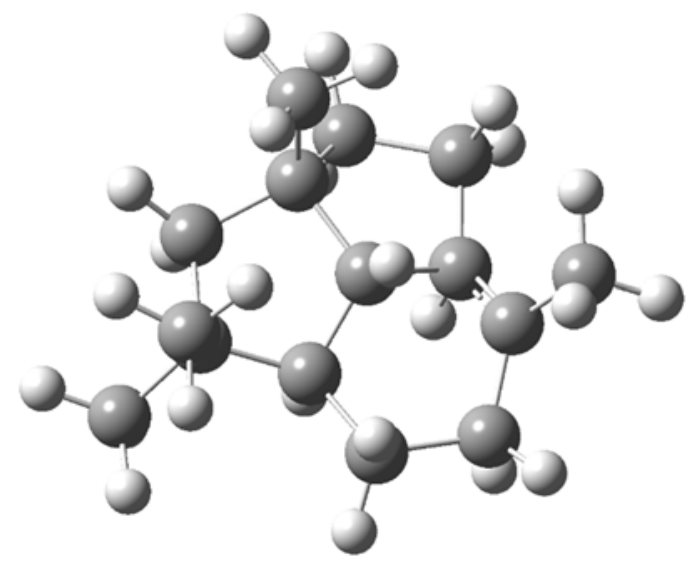

M06-2X/ 6-311++g(2d,p)

$\mathrm{HF}=-585.936674$ hartree $(-367680.5364 \mathrm{Kcal} / \mathrm{mole})$

M06-2X/ 6-31+g(d,p)

$\mathrm{HF}=-585.803561$ hartree $(-367597.0068 \mathrm{kcal} / \mathrm{mole})$

Standard orientation:

$\begin{array}{rrrrr}1 & 6 & -0.682240 & -0.907383 & -0.462094 \\ 2 & 6 & -0.176873 & -2.280631 & -0.042525 \\ 3 & 6 & 1.356271 & -2.317556 & -0.391599 \\ 4 & 6 & 2.007141 & -0.998136 & -0.047868 \\ 5 & 6 & 1.660458 & 0.115491 & -0.846082 \\ 6 & 6 & 0.313408 & 0.063954 & 0.119091 \\ 7 & 1 & 1.849028 & -3.130484 & 0.139665 \\ 8 & 1 & -0.299179 & -2.417639 & 1.034849 \\ 9 & 1 & -0.669809 & -3.109965 & -0.547497 \\ 10 & 1 & -0.653911 & -0.841580 & -1.555353\end{array}$




$\begin{array}{lrrrr}11 & 1 & 1.296704 & -0.153973 & -1.833402 \\ 12 & 1 & 1.463273 & -2.487303 & -1.465002 \\ 13 & 6 & 2.056419 & 1.576484 & -0.777378 \\ 14 & 1 & 2.753421 & 1.834591 & -1.574356 \\ 15 & 1 & 2.518605 & 1.835719 & 0.175810 \\ 16 & 6 & 0.679554 & 2.257395 & -0.928873 \\ 17 & 6 & -0.229855 & 1.523123 & 0.081865 \\ 18 & 6 & -2.023341 & -0.266028 & -0.037054 \\ 19 & 6 & -1.707952 & 1.239787 & -0.322783 \\ 20 & 1 & 0.318375 & 2.110546 & -1.950240 \\ 21 & 1 & 0.722798 & 3.330430 & -0.745564 \\ 22 & 1 & -2.401947 & 1.900143 & 0.200505 \\ 23 & 1 & -1.832021 & 1.418861 & -1.394197 \\ 24 & 6 & -0.131023 & 2.203720 & 1.447583 \\ 25 & 1 & -0.550970 & 3.208599 & 1.383717 \\ 26 & 1 & -0.684844 & 1.662106 & 2.214244 \\ 27 & 1 & 0.906603 & 2.297817 & 1.777471 \\ 28 & 1 & 0.505176 & -0.203406 & 1.164739 \\ 29 & 6 & -2.346993 & -0.531265 & 1.435418 \\ 30 & 1 & -3.191711 & 0.083216 & 1.751176 \\ 31 & 1 & -2.627965 & -1.576619 & 1.579142 \\ 32 & 1 & -1.515927 & -0.318871 & 2.110348 \\ 33 & 6 & -3.184916 & -0.750134 & -0.899454 \\ 34 & 1 & -4.101175 & -0.216311 & -0.639744 \\ 35 & 1 & -2.987648 & -0.584757 & -1.960585 \\ 36 & 1 & -3.367558 & -1.815715 & -0.745398 \\ 37 & 6 & 2.913579 & -0.928153 & 1.121184 \\ 38 & 1 & 3.120003 & 0.087963 & 1.448637 \\ 39 & 1 & 2.550792 & -1.540477 & 1.947675 \\ 40 & 1 & 3.858082 & -1.376899 & 0.786774\end{array}$




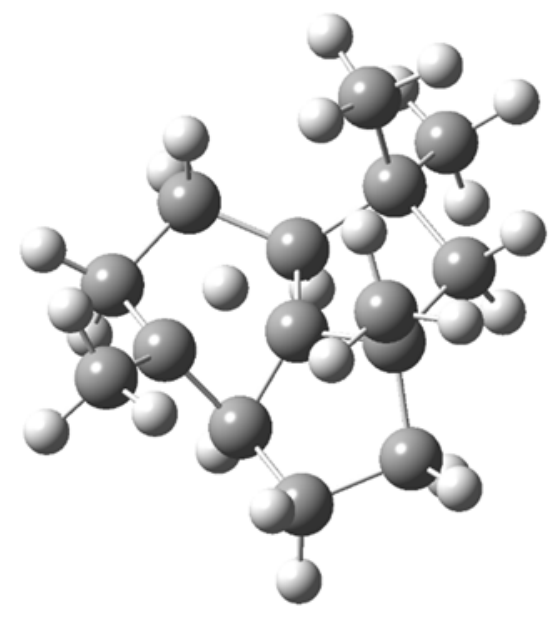

M06-2X/ 6-311++g(2d,p)

$\mathrm{HF}=-585.919668$ hartree $(-367669.8649 \mathrm{kcal} / \mathrm{mole})$

M06-2X/ 6-31+g(d,p)

$\mathrm{HF}=-585.7876761$ hartree $(-367587.0388 \mathrm{kcal} / \mathrm{mole})$

Standard orientation:

$\begin{array}{rrrrr}1 & 6 & 0.865610 & 0.813113 & -0.642607 \\ 2 & 6 & 0.498852 & 2.248851 & -0.211788 \\ 3 & 6 & -1.039319 & 2.399135 & -0.292361 \\ 4 & 6 & -1.675021 & 1.059642 & 0.115456 \\ 5 & 6 & -1.594213 & 0.025880 & -1.025900 \\ 6 & 6 & -0.311410 & -0.052116 & -0.322892 \\ 7 & 1 & -1.383620 & 3.189732 & 0.373539 \\ 8 & 1 & 0.803870 & 2.398032 & 0.826016 \\ 9 & 1 & 1.005603 & 3.000637 & -0.813118 \\ 10 & 1 & 0.969359 & 0.782727 & -1.734288 \\ 11 & 1 & -1.593974 & 0.492815 & -2.004515 \\ 12 & 1 & -1.362567 & 2.651723 & -1.303510 \\ 13 & 6 & -2.178415 & -1.392346 & -0.920820 \\ 14 & 1 & -2.750220 & -1.651562 & -1.809279 \\ 15 & 1 & -2.850072 & -1.495733 & -0.068384 \\ 16 & 6 & -0.918770 & -2.275590 & -0.746364 \\ 17 & 6 & 0.011132 & -1.445422 & 0.181005 \\ 18 & 6 & 2.053066 & 0.024910 & -0.013387 \\ 19 & 6 & 1.541472 & -1.449088 & -0.092833 \\ 20 & 1 & -0.440705 & -2.416863 & -1.718634 \\ 21 & 1 & -1.141925 & -3.260486 & -0.337428 \\ 22 & 1 & 2.076789 & -2.098676 & 0.601277\end{array}$




$\begin{array}{rrrrr}23 & 1 & 1.722763 & -1.828022 & -1.102205 \\ 24 & 6 & -0.335667 & -1.732243 & 1.645793 \\ 25 & 1 & -0.052418 & -2.759207 & 1.877136 \\ 26 & 1 & 0.192141 & -1.080011 & 2.339355 \\ 27 & 1 & -1.408744 & -1.639056 & 1.827072 \\ 28 & 1 & -0.796727 & 0.618043 & 0.838180 \\ 29 & 6 & 2.325507 & 0.456662 & 1.430639 \\ 30 & 1 & 2.999306 & -0.253727 & 1.911801 \\ 31 & 1 & 2.807341 & 1.435849 & 1.453498 \\ 32 & 1 & 1.424434 & 0.525996 & 2.043440 \\ 33 & 6 & 3.330747 & 0.196927 & -0.830296 \\ 34 & 1 & 4.138363 & -0.404315 & -0.408480 \\ 35 & 1 & 3.184472 & -0.114046 & -1.866600 \\ 36 & 1 & 3.656699 & 1.239179 & -0.827414 \\ 37 & 6 & -2.858599 & 1.040597 & 1.066626 \\ 38 & 1 & -3.119531 & 0.036634 & 1.391573 \\ 39 & 1 & -2.659788 & 1.657492 & 1.941567 \\ 40 & 1 & -3.710674 & 1.459379 & 0.527779\end{array}$

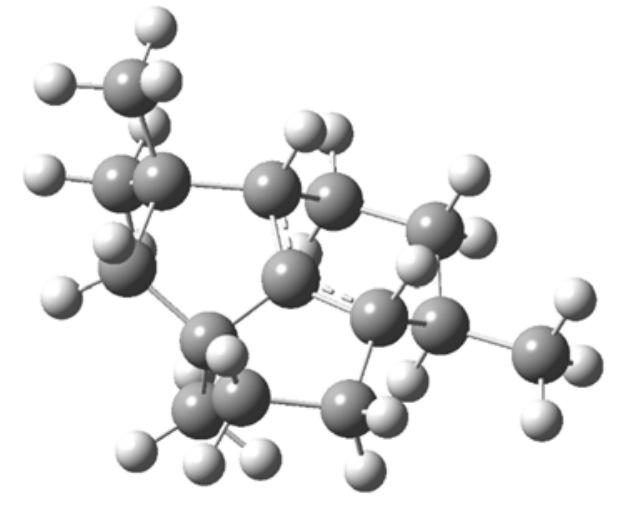

M06-2X/ 6-311++g(2d,p)

$\mathrm{HF}=-585.942734$ hartree $(-367684.3391 \mathrm{kcal} / \mathrm{mole})$

M06-2X/ 6-31+g(d,p)

$\mathrm{HF}=-585.809811 \mathrm{hartree}(-367600.9287 \mathrm{kcal} / \mathrm{mole})$

Standard orientation:

$\begin{array}{rrrrr}1 & 6 & -0.734046 & -0.906573 & -0.498823 \\ 2 & 6 & -0.037685 & -1.967022 & 0.454480\end{array}$




$\begin{array}{rrrrr}3 & 6 & 1.447659 & -2.096309 & 0.109665 \\ 4 & 6 & 2.219290 & -0.778928 & 0.243321 \\ 5 & 6 & 1.552443 & 0.316956 & -0.694609 \\ 6 & 6 & 0.153363 & 0.215039 & -0.369076 \\ 7 & 1 & 1.902230 & -2.834369 & 0.774536 \\ 8 & 1 & -0.162943 & -1.660020 & 1.493997 \\ 9 & 1 & -0.566632 & -2.911131 & 0.325249 \\ 10 & 1 & -0.658985 & -1.346277 & -1.500050 \\ 11 & 1 & 1.744486 & -0.024920 & -1.717724 \\ 12 & 1 & 1.563276 & -2.481887 & -0.908767 \\ 13 & 6 & 1.874699 & 1.813671 & -0.466777 \\ 14 & 1 & 2.494725 & 2.225549 & -1.259647 \\ 15 & 1 & 2.420561 & 1.944937 & 0.469270 \\ 16 & 6 & 0.479679 & 2.496546 & -0.403424 \\ 17 & 6 & -0.427448 & 1.407435 & 0.232990 \\ 18 & 6 & -2.164187 & -0.335637 & -0.208247 \\ 19 & 6 & -1.924521 & 1.215345 & -0.106757 \\ 20 & 1 & 0.129169 & 2.732254 & -1.410581 \\ 21 & 1 & 0.481051 & 3.418304 & 0.176759 \\ 22 & 1 & -2.574437 & 1.678356 & 0.636247 \\ 23 & 1 & -2.139976 & 1.682572 & -1.070584 \\ 24 & 6 & -0.189874 & 1.400456 & 1.773770 \\ 25 & 1 & -0.693397 & 2.284501 & 2.165492 \\ 26 & 1 & -0.611310 & 0.522624 & 2.256444 \\ 27 & 1 & 0.868047 & 1.467381 & 2.021417 \\ 28 & 6 & -2.797552 & -0.911119 & 1.060827 \\ 29 & 1 & -3.799205 & -0.497161 & 1.184949 \\ 30 & 1 & -2.897856 & -1.995612 & 0.984930 \\ 31 & 1 & -2.238334 & -0.688238 & 1.968706 \\ 32 & 6 & -3.083895 & -0.645511 & -1.390565 \\ 33 & 1 & -4.052522 & -0.161889 & -1.252951 \\ 34 & 1 & -2.659823 & -0.286384 & -2.330929 \\ 35 & 1 & -3.255960 & -1.720375 & -1.479205 \\ 36 & 6 & 3.694975 & -0.907261 & -0.106972 \\ 37 & 1 & 3.816261 & -1.287773 & -1.123159 \\ 38 & 1 & 4.205204 & 0.053990 & -0.036005 \\ 39 & 1 & 4.184490 & -1.598822 & 0.579631 \\ 40 & 1 & 2.124488 & -0.418145 & 1.273156\end{array}$




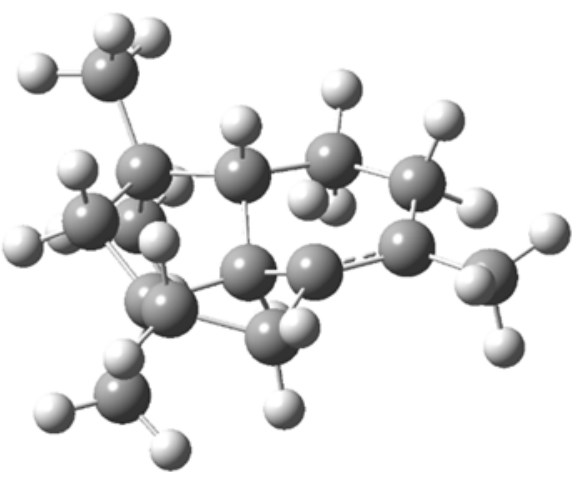

M06-2X/ 6-311++g(2d,p)

$\mathrm{HF}=-585.924906$ hartree $(-367673.1518 \mathrm{kcal} / \mathrm{mole})$

M06-2X/ 6-31+g(d,p)

$\mathrm{HF}=-586.114601$ hartree $(-367792.1872 \mathrm{kcal} / \mathrm{mole})$

Standard orientation:

$\begin{array}{rrrrr}1 & 6 & -0.508355 & -0.970123 & -0.279516 \\ 2 & 6 & 0.185744 & -2.191679 & 0.321257 \\ 3 & 6 & 1.735278 & -2.051329 & 0.188774 \\ 4 & 6 & 2.381115 & -0.711529 & 0.047218 \\ 5 & 6 & 1.533553 & 0.409621 & -0.244680 \\ 6 & 6 & 0.155770 & 0.230521 & 0.356729 \\ 7 & 1 & 2.269336 & -2.578122 & 0.989486 \\ 8 & 1 & -0.068350 & -2.275768 & 1.380111 \\ 9 & 1 & -0.108199 & -3.126931 & -0.156355 \\ 10 & 1 & -0.285302 & -0.954741 & -1.356386 \\ 11 & 1 & 1.448230 & 0.047446 & -1.316027 \\ 12 & 1 & 2.098173 & -2.574099 & -0.714023 \\ 13 & 6 & 1.742138 & 1.915784 & -0.375773 \\ 14 & 1 & 2.526721 & 2.207094 & -1.073378 \\ 15 & 1 & 1.962261 & 2.354986 & 0.599685 \\ 16 & 6 & 0.314874 & 2.311457 & -0.855528 \\ 17 & 6 & -0.661542 & 1.491058 & 0.038748 \\ 18 & 6 & -2.008306 & -0.629843 & -0.188086 \\ 19 & 6 & -1.944035 & 0.880534 & -0.602332 \\ 20 & 1 & 0.209636 & 2.028642 & -1.907474 \\ 21 & 1 & 0.144693 & 3.385123 & -0.786711 \\ 22 & 1 & -2.847918 & 1.415278 & -0.304656 \\ 23 & 1 & -1.883135 & 0.933525 & -1.693485 \\ 24 & 6 & -0.989957 & 2.309488 & 1.288932 \\ 25 & 1 & -1.563282 & 3.196485 & 1.014593\end{array}$




$\begin{array}{lrrrr}26 & 1 & -1.584110 & 1.738204 & 2.002114 \\ 27 & 1 & -0.081959 & 2.643560 & 1.798120 \\ 28 & 1 & 0.253033 & 0.083414 & 1.438333 \\ 29 & 6 & -2.553600 & -0.831974 & 1.228014 \\ 30 & 1 & -3.551323 & -0.398422 & 1.315736 \\ 31 & 1 & -2.637785 & -1.897491 & 1.453754 \\ 32 & 1 & -1.926562 & -0.380178 & 1.997992 \\ 33 & 6 & -2.852017 & -1.434355 & -1.171612 \\ 34 & 1 & -3.890731 & -1.098927 & -1.150887 \\ 35 & 1 & -2.484037 & -1.323213 & -2.194102 \\ 36 & 1 & -2.844630 & -2.496714 & -0.917149 \\ 37 & 6 & 3.840352 & -0.608989 & 0.094216 \\ 38 & 1 & 4.213995 & 0.177006 & -0.563206 \\ 39 & 1 & 4.050392 & -0.261993 & 1.120727 \\ 40 & 1 & 4.354776 & -1.556004 & -0.054971\end{array}$

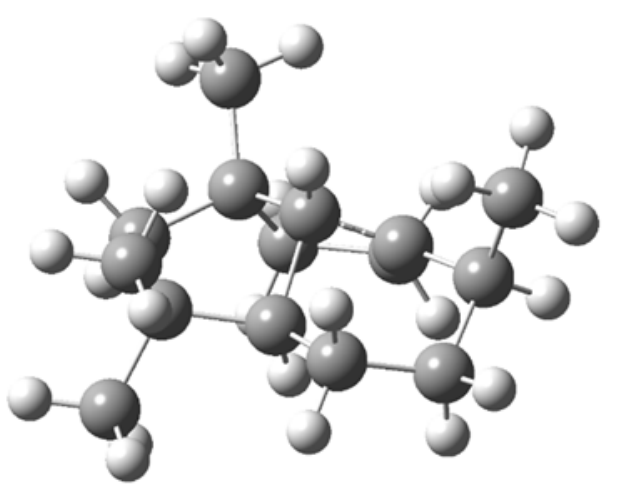

M06-2X/ 6-311++g(2d,p)

$\mathrm{HF}=-585.955226$ hartree $(-367692.1779 \mathrm{kcal} / \mathrm{mole})$

$\mathrm{M} 06-2 \mathrm{X} / 6-31+\mathrm{g}(\mathrm{d}, \mathrm{p})$

$\mathrm{HF}=-585.82183$ hartree $(-367608.4707 \mathrm{kcal} / \mathrm{mole})$

Standard orientation:

$\begin{array}{rrrrr}1 & 6 & -0.240682 & -0.849321 & -0.351926 \\ 2 & 6 & 0.719928 & -1.993177 & -0.096349 \\ 3 & 6 & 2.084867 & -1.605056 & -0.665885 \\ 4 & 6 & 2.562811 & -0.194780 & -0.144186 \\ 5 & 6 & 1.374582 & 0.667960 & -0.239934 \\ 6 & 6 & 0.209485 & 0.442112 & 0.535008 \\ 7 & 1 & 2.862238 & -2.322647 & -0.401947 \\ 8 & 1 & 0.777076 & -2.224498 & 0.969278 \\ 9 & 1 & 0.363626 & -2.894216 & -0.602741\end{array}$




$\begin{array}{lrrrr}10 & 1 & -0.182431 & -0.593586 & -1.416410 \\ 11 & 1 & 3.338236 & 0.170231 & -0.819396 \\ 12 & 1 & 2.023873 & -1.570793 & -1.755724 \\ 13 & 6 & 1.127298 & 1.739903 & -1.221162 \\ 14 & 1 & 1.576145 & 1.558755 & -2.199685 \\ 15 & 1 & 1.679805 & 2.594762 & -0.791774 \\ 16 & 6 & -0.392388 & 1.998468 & -1.184967 \\ 17 & 6 & -0.885182 & 1.454794 & 0.188627 \\ 18 & 6 & -1.749343 & -0.899361 & 0.010086 \\ 19 & 6 & -2.171600 & 0.597236 & 0.078078 \\ 20 & 1 & -0.865795 & 1.465403 & -2.010492 \\ 21 & 1 & -0.628605 & 3.054248 & -1.306682 \\ 22 & 1 & -2.817036 & 0.759634 & 0.943120 \\ 23 & 1 & -2.747150 & 0.886848 & -0.802859 \\ 24 & 6 & -0.988234 & 2.570483 & 1.222517 \\ 25 & 1 & -1.801001 & 3.250701 & 0.963281 \\ 26 & 1 & -1.193312 & 2.164353 & 2.214911 \\ 27 & 1 & -0.066499 & 3.155817 & 1.276654 \\ 28 & 1 & 0.315570 & 0.100970 & 1.561254 \\ 29 & 6 & -1.961780 & -1.584456 & 1.361259 \\ 30 & 1 & -3.026300 & -1.587986 & 1.600123 \\ 31 & 1 & -1.623909 & -2.622161 & 1.340017 \\ 32 & 1 & -1.450635 & -1.069714 & 2.178413 \\ 33 & 6 & -2.504442 & -1.663320 & -1.078048 \\ 34 & 1 & -3.573210 & -1.665007 & -0.857233 \\ 35 & 1 & -2.365022 & -1.200348 & -2.057469 \\ 36 & 1 & -2.176735 & -2.703429 & -1.134703 \\ 37 & 6 & 3.113208 & -0.299479 & 1.285768 \\ 38 & 1 & 3.337438 & 0.682644 & 1.702110 \\ 39 & 1 & 2.414920 & -0.806441 & 1.951194 \\ 40 & 1 & 4.037556 & -0.875574 & 1.263451 \\ & & & & \\ 13 & 1 & 1 & \end{array}$




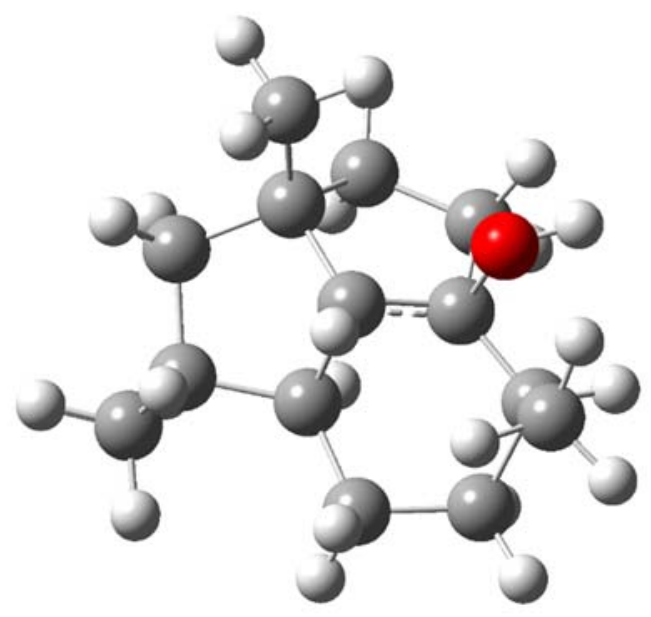

M06-2X/ 6-311++g(2d,p)

$\mathrm{HF}=-662.041067$ hartree $(-415436.7279 \mathrm{kcal} / \mathrm{mole})$

$\mathrm{M} 06-2 \mathrm{X} / 6-31+\mathrm{g}(\mathrm{d}, \mathrm{p})$

$\mathrm{HF}=--661.884455$ hartree $(-415338.4525 \mathrm{kcal} / \mathrm{mole})$

Standard orientation:

$\begin{array}{rrrrr}1 & 6 & 0.000000 & 0.000000 & 0.000000 \\ 2 & 6 & 0.000000 & 0.000000 & 1.515420 \\ 3 & 6 & 1.453028 & 0.000000 & 1.991631 \\ 4 & 6 & 2.294956 & 1.151945 & 1.318088 \\ 5 & 6 & 1.912310 & 1.120862 & -0.102473 \\ 6 & 6 & 0.597114 & 1.417434 & -0.539849 \\ 7 & 1 & 1.534536 & 0.134828 & 3.070558 \\ 8 & 1 & -0.553790 & 0.856227 & 1.905968 \\ 9 & 1 & -0.497779 & -0.900787 & 1.884274 \\ 10 & 1 & 0.650269 & -0.816346 & -0.335633 \\ 11 & 1 & 3.352051 & 0.901864 & 1.420233 \\ 12 & 1 & 1.913449 & -0.958639 & 1.743303 \\ 13 & 6 & 2.721738 & 0.633120 & -1.233824 \\ 14 & 1 & 3.394560 & -0.187517 & -0.977600 \\ 15 & 1 & 3.373746 & 1.496690 & -1.456435 \\ 16 & 6 & 1.726738 & 0.388966 & -2.386201 \\ 17 & 6 & 0.472158 & 1.250678 & -2.056549 \\ 18 & 6 & -1.291427 & -0.080769 & -0.857501 \\ 19 & 6 & -0.860784 & 0.481996 & -2.243347 \\ 20 & 1 & 1.475330 & -0.671785 & -2.423151 \\ 21 & 1 & 2.151540 & 0.652395 & -3.353379 \\ 22 & 1 & -1.636329 & 1.146020 & -2.629172 \\ 23 & 1 & -0.738880 & -0.318307 & -2.975356 \\ 24 & 6 & 0.508380 & 2.586932 & -2.789641\end{array}$




$\begin{array}{rrrrr}25 & 1 & 0.379644 & 2.432149 & -3.862003 \\ 26 & 1 & -0.294447 & 3.241936 & -2.445718 \\ 27 & 1 & 1.459682 & 3.103832 & -2.638049 \\ 28 & 1 & 0.040238 & 2.206658 & -0.042028 \\ 29 & 6 & -2.403622 & 0.774023 & -0.247169 \\ 30 & 1 & -3.291098 & 0.715405 & -0.879009 \\ 31 & 1 & -2.680817 & 0.417875 & 0.746693 \\ 32 & 1 & -2.130287 & 1.829431 & -0.173878 \\ 33 & 6 & -1.750272 & -1.536787 & -0.943021 \\ 34 & 1 & -2.631836 & -1.611575 & -1.581989 \\ 35 & 1 & -0.972040 & -2.173736 & -1.369281 \\ 36 & 1 & -2.020302 & -1.927568 & 0.040240 \\ 37 & 6 & 2.004244 & 2.503419 & 1.987170 \\ 38 & 1 & 2.487386 & 3.324976 & 1.458206 \\ 39 & 1 & 0.934927 & 2.706494 & 2.039417 \\ 40 & 1 & 2.394107 & 2.481246 & 3.004184 \\ 41 & 8 & 2.367699 & 2.465712 & -0.272465 \\ 42 & 1 & 3.325558 & 2.489624 & -0.213015\end{array}$

(-)-presilphiperfolan-8 $\alpha$-ol (-)-3

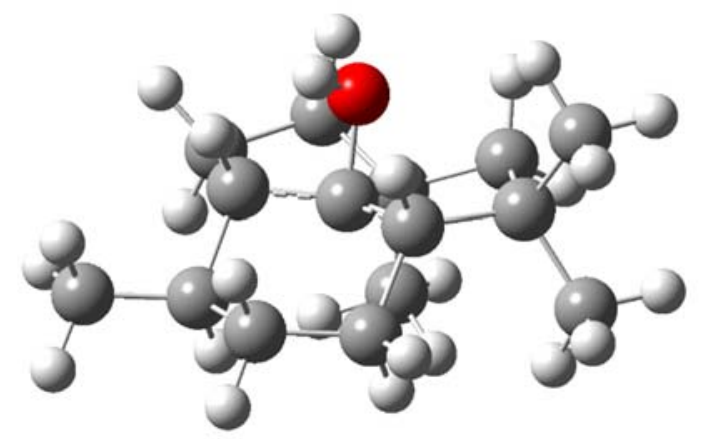

M06-2X/ 6-311++g(2d,p)

$\mathrm{HF}=-662.021014$ hartree $(-415424.1445 \mathrm{kcal} / \mathrm{mole})$

M06-2X/ 6-31+g(d,p)

$\mathrm{HF}=--661.864662$ hartree $(-415326.0322 \mathrm{kcal} / \mathrm{mole})$

Standard orientation:

$\begin{array}{rrrrr}1 & 6 & 0.000000 & 0.000000 & 0.000000 \\ 2 & 6 & 0.000000 & 0.000000 & 1.586902 \\ 3 & 6 & 1.435216 & 0.000000 & 2.117952 \\ 4 & 6 & 2.253755 & 1.210716 & 1.656508 \\ 5 & 6 & 2.277967 & 1.252842 & 0.068113 \\ 6 & 6 & 0.890599 & 1.090669 & -0.282164 \\ 7 & 1 & 1.408323 & -0.005557 & 3.210045\end{array}$




$\begin{array}{rrrrr}8 & 1 & -0.541851 & 0.874175 & 1.951253 \\ 9 & 1 & -0.550159 & -0.884313 & 1.908059 \\ 10 & 1 & 0.459482 & -0.956476 & -0.274698 \\ 11 & 1 & 2.865024 & 0.378340 & -0.233774 \\ 12 & 1 & 1.946551 & -0.919631 & 1.814545 \\ 13 & 6 & 2.689029 & 2.558903 & -0.653791 \\ 14 & 1 & 3.661505 & 2.474494 & -1.133253 \\ 15 & 1 & 2.753006 & 3.381890 & 0.060338 \\ 16 & 6 & 1.549825 & 2.797298 & -1.684225 \\ 17 & 6 & 0.292754 & 2.225614 & -0.972174 \\ 18 & 6 & -1.292542 & 0.297624 & -0.834542 \\ 19 & 6 & -0.886578 & 1.543033 & -1.704849 \\ 20 & 1 & 1.746876 & 2.236721 & -2.600579 \\ 21 & 1 & 1.427551 & 3.846157 & -1.951054 \\ 22 & 1 & -1.724555 & 2.224678 & -1.853105 \\ 23 & 1 & -0.555335 & 1.208048 & -2.690622 \\ 24 & 6 & -0.213706 & 3.276222 & 0.062337 \\ 25 & 1 & -0.695289 & 4.066386 & -0.514062 \\ 26 & 1 & -0.942664 & 2.862180 & 0.753974 \\ 27 & 1 & 0.605366 & 3.713465 & 0.630619 \\ 28 & 6 & -2.522633 & 0.570721 & 0.034466 \\ 29 & 1 & -3.388978 & 0.737643 & -0.607141 \\ 30 & 1 & -2.745510 & -0.288880 & 0.669571 \\ 31 & 1 & -2.419573 & 1.444824 & 0.676313 \\ 32 & 6 & -1.596887 & -0.896071 & -1.741309 \\ 33 & 1 & -2.429705 & -0.662606 & -2.406872 \\ 34 & 1 & -0.735462 & -1.157077 & -2.360113 \\ 35 & 1 & -1.874972 & -1.772384 & -1.151784 \\ 36 & 6 & 3.683227 & 1.205764 & 2.179391 \\ 37 & 1 & 4.199267 & 0.292497 & 1.876435 \\ 38 & 1 & 4.246697 & 2.060533 & 1.803564 \\ 39 & 1 & 3.684081 & 1.255691 & 3.268800 \\ 40 & 1 & 1.751411 & 2.124222 & 1.992468 \\ 41 & 8 & 1.273703 & 0.458503 & -1.506296 \\ 42 & 1 & 1.896302 & -0.248292 & -1.320791\end{array}$




\section{LDS224_130K}

Submitted by: Leonidas Dimitrios Syntrivanis, University of Basel

Report created with ReportPlus

Solved by: Alessandro Prescimone

Sample ID: $\quad$ LDS224_130K

Crystal Data and Experimental

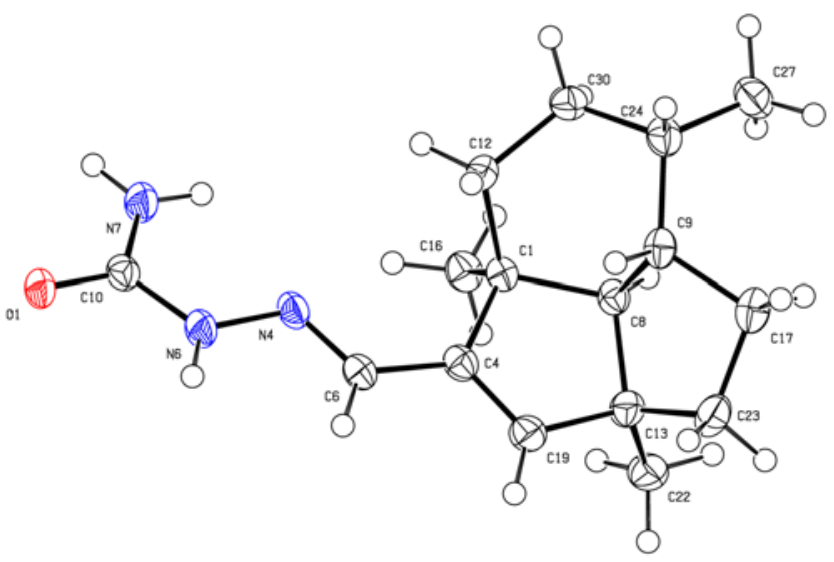

Experimental. Single colourless block-shaped crystals of LDS224_130K were recrystallised from ethanol by vapour diffusion of hexane. A suitable crystal $0.38 \times 0.32 \times 0.21 \mathrm{~mm}^{3}$ was selected and mounted on a mylar loop in perfluoroether oil on a Bruker Kappa Apex2 diffractometer. The crystal was kept at a steady $T=130 \mathrm{~K}$ during data collection. The structure was solved with the Superflip (Palatinus \& Chapuis, 2007;Palatinus \& van der Lee, 2008;Palatinus et al., 2012) structure solution program using the Charge Flipping solution method and by using Olex2 (Dolomanov et al., 2009) as the graphical interface. The model was refined with version 2014/7 of ShelXL (Sheldrick, 2015) using Least Squares minimisation.

Crystal Data. $\mathrm{C}_{16} \mathrm{H}_{25} \mathrm{~N}_{3} \mathrm{O}, M_{r}=275.39$, monoclinic, $C 2$ (No. 5), $\mathrm{a}=20.475(2) \AA ̊ . \mathrm{b}=7.6092(8) \AA, \mathrm{c}=12.4167(13) \AA, \beta=$ $124.988(2)^{\circ}, \alpha=\gamma=90^{\circ}, V=1584.9(3) \AA^{3}, T=130 \mathrm{~K}, Z=4$, $Z^{\prime}=1, \mu\left(\mathrm{CuK}_{\alpha}\right)=0.575,10053$ reflections measured, 2855 unique $\left(R_{\text {int }}=0.0248\right)$ which were used in all calculations. The final $w R_{2}$ was 0.0740 (all data) and $R_{1}$ was 0.0280 (I > 2(I)).

\begin{tabular}{|c|c|}
\hline Compound & LDS224_130K \\
\hline Formula & $\mathrm{C}_{16} \mathrm{H}_{25} \mathrm{~N}_{3} \mathrm{O}$ \\
\hline$D_{\text {calc. }} / \mathrm{g} \mathrm{cm}^{-3}$ & 1.154 \\
\hline$\mu / \mathrm{mm}^{-1}$ & 0.575 \\
\hline Formula Weight & 275.39 \\
\hline Colour & colourless \\
\hline Shape & block \\
\hline Size $/ \mathrm{mm}^{3}$ & $0.38 \times 0.32 \times 0.21$ \\
\hline$T / \mathrm{K}$ & 130 \\
\hline Crystal System & monoclinic \\
\hline Flack Parameter & $0.07(5)$ \\
\hline Hooft Parameter & $0.08(5)$ \\
\hline Space Group & $C 2$ \\
\hline$a / \AA$ & $20.475(2)$ \\
\hline$b / \AA$ & $7.6092(8)$ \\
\hline$c / \AA ̊$ & $12.4167(13)$ \\
\hline$\left.\alpha\right|^{\circ}$ & 90 \\
\hline$\beta 1^{\circ}$ & $124.988(2)$ \\
\hline$\gamma /^{\circ}$ & 90 \\
\hline $\mathrm{V} / \AA^{3}$ & $1584.9(3)$ \\
\hline$Z$ & 4 \\
\hline$Z^{\prime}$ & 1 \\
\hline Wavelength/Å & 1.541838 \\
\hline Radiation type & $\mathrm{CuK}_{\alpha}$ \\
\hline$\Theta_{\min } /^{\circ}$ & 4.346 \\
\hline$\left.\Theta_{\max }\right|^{\circ}$ & 69.909 \\
\hline Measured Refl. & 10053 \\
\hline Independent Refl. & 2855 \\
\hline $\begin{array}{l}\text { Reflections with I > } \\
2(\mathrm{I})\end{array}$ & 2849 \\
\hline$R_{\text {int }}$ & 0.0248 \\
\hline Parameters & 184 \\
\hline Restraints & 1 \\
\hline Largest Peak & 0.181 \\
\hline Deepest Hole & -0.121 \\
\hline GooF & 1.057 \\
\hline$w R_{2}$ (all data) & 0.0740 \\
\hline$w R_{2}$ & 0.0739 \\
\hline$R_{1}$ (all data) & 0.0280 \\
\hline$R_{1}$ & 0.0280 \\
\hline
\end{tabular}




\section{Structure Quality Indicators}

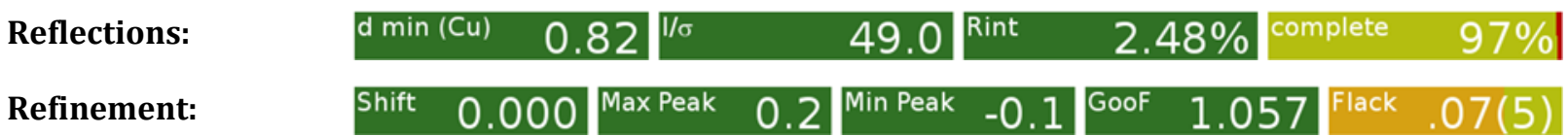

A colourless block-shaped crystal with dimensions $0.38 \times 0.32 \times 0.21 \mathrm{~mm}^{3}$ was mounted on a mylar loop in perfluoroether oil. Data were collected using a Bruker Kappa Apex2 diffractometer equipped with an Oxford Cryosystems low-temperature device operating at $T=130 \mathrm{~K}$.

Data were measured using $\phi \& \omega$ scans using $\mathrm{CuK}_{\alpha}$ radiation. The total number of runs and images was based on the strategy calculation from the program APEX2 (Bruker, V2, n/a) The maximum resolution that was achieved was $\Theta=69.909^{\circ}(0.82 \AA)$.

The diffraction pattern was indexed The total number of runs and images was based on the strategy calculation from the program APEX2 (Bruker, V2, n/a) and the unit cell was refined using SAINT (Bruker, V8.34A, after 2013) on 9987 reflections, $99 \%$ of the observed reflections.

Data reduction, scaling and absorption corrections were performed using SAINT (Bruker, V8.34A, after 2013). The final completeness is $98.30 \%$ out to $69.909^{\circ}$ in $\Theta$. An absorption correction was performed using SADABS-2012/1 (Bruker,2012) was used for absorption correction. $w R_{2}$ (int) was 0.0891 before and 0.0374 after correction. The Ratio of minimum to maximum transmission is 0.9034 . The $\lambda / 2$ correction factor is 0.0015 .. The absorption coefficient $\mu$ of this material is $0.575 \mathrm{~mm}^{-1}$ at this wavelength $(\lambda=1.542 \AA)$ and the minimum and maximum transmissions are 0.680 and 0.753 .

The structure was solved and the space group C2 (\# 5) determined by the Superflip (Palatinus \& Chapuis, 2007; Palatinus \& van der Lee, 2008;Palatinus et al., 2012) structure solution program using Charge Flipping and refined by Least Squares using version 2014/7 of ShelXL (Sheldrick, 2015). All nonhydrogen atoms were refined anisotropically. Hydrogen atom positions were calculated geometrically and refined using the riding model. Hydrogen atom positions were calculated geometrically and refined using the riding model.

_exptl_absorpt_process_details: SADABS-2012/1 (Bruker,2012) was used for absorption correction. $w R_{2}$ (int) was 0.0891 before and 0.0374 after correction. The Ratio of minimum to maximum transmission is 0.9034 . The $\lambda / 2$ correction factor is 0.0015 .

\section{Data Plots: Diffraction Data}
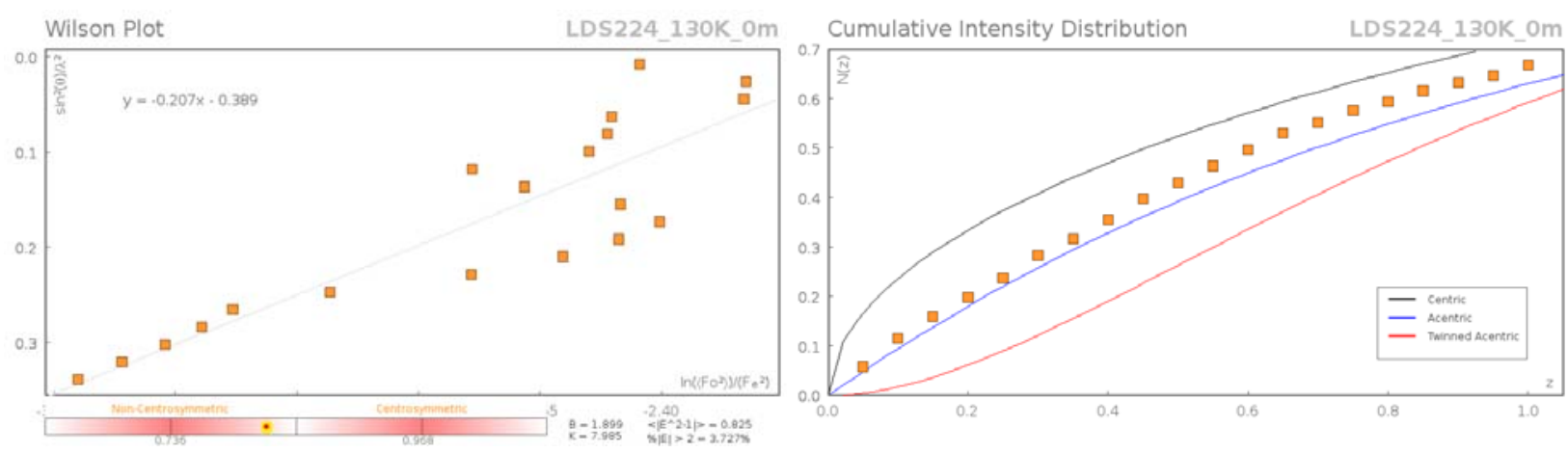

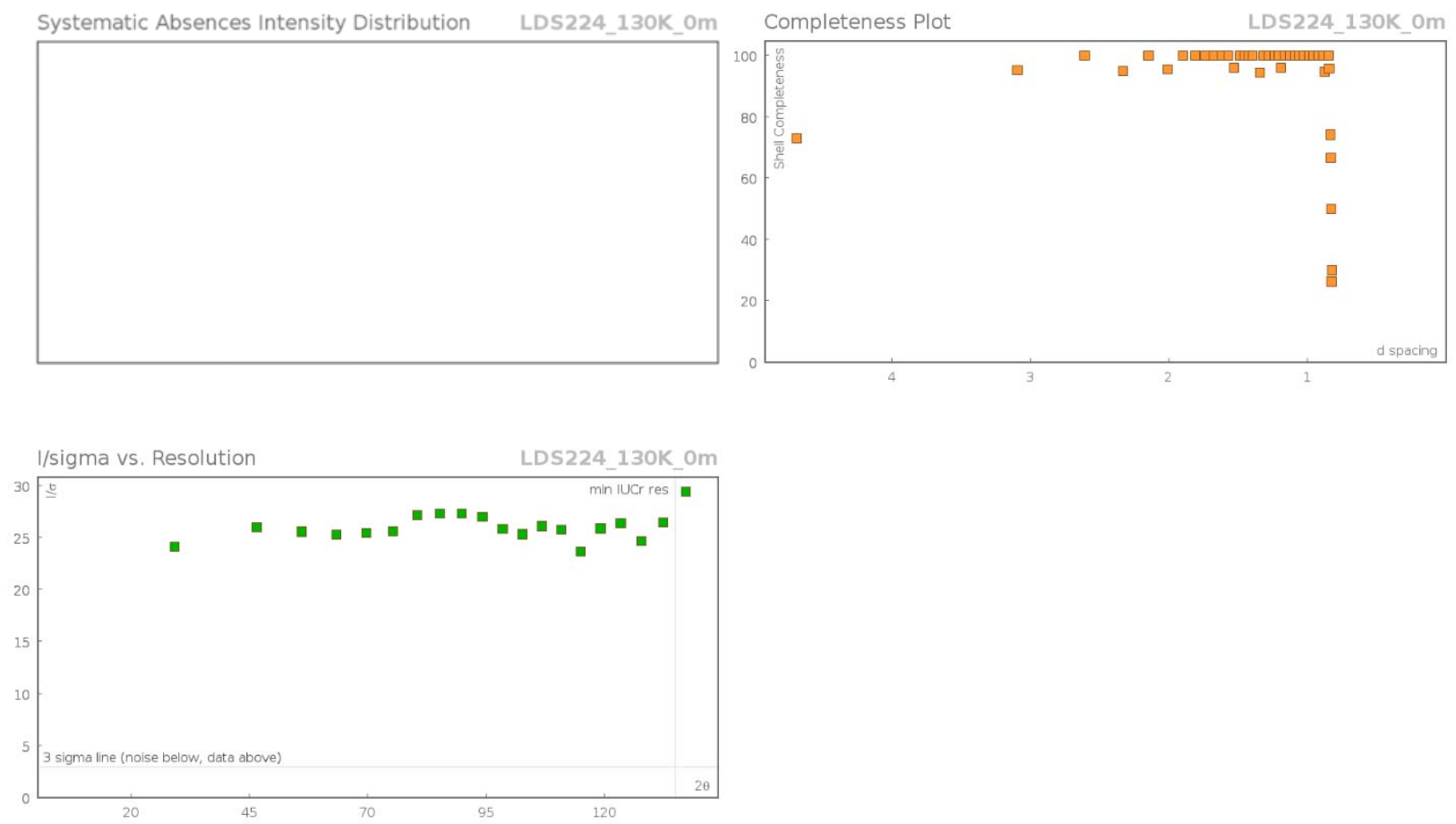

\section{Data Plots: Refinement and Data}
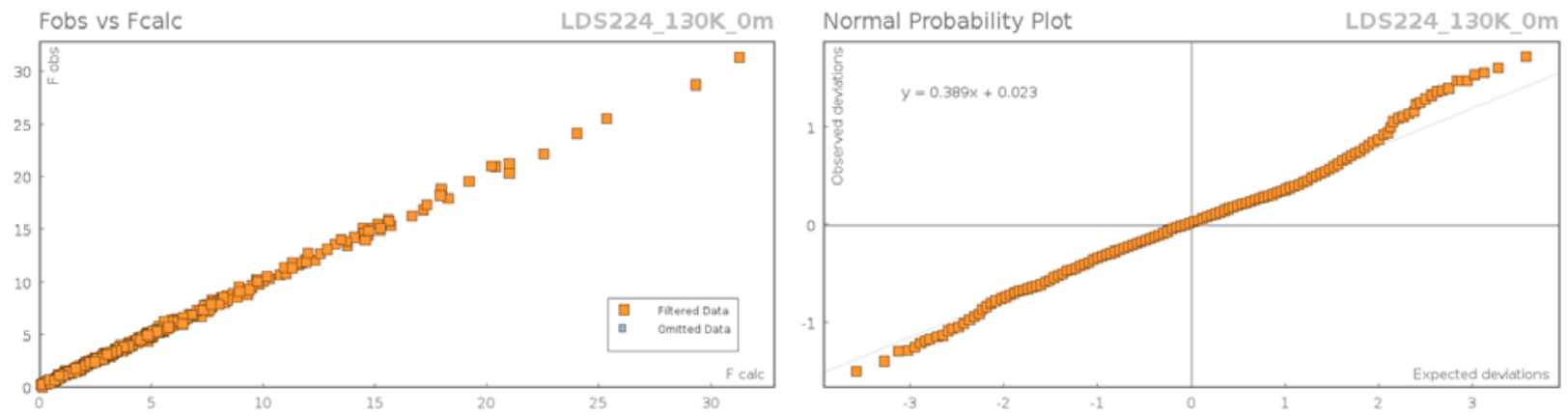

\section{Reflection Statistics}

Total reflections (after

filtering)

Completeness

$\mathrm{hkl}_{\max }$ collected

hklmax used

Lim $d_{\text {max }}$ collected

$\mathrm{d}_{\max }$ used

Friedel pairs

Inconsistent equivalents

Rsigma

Omitted reflections

Multiplicity
10053

0.949

$(24,9,13)$

$(20,9,15)$

100.0

10.17

1510

1

0.0204

0

(1710, 1116, 754, 390, 249, $108,37,16,1)$

Removed systematic absences 0

$\begin{array}{ll}\text { Unique reflections } & 2855 \\ \text { Mean I/ } \sigma & 45.17 \\ \mathrm{hkl}_{\text {min }} \text { collected } & (-23,-9,-15) \\ \mathrm{hkl}_{\text {min }} \text { used } & (-24,-9,0) \\ \text { Lim }_{\text {min }} \text { collected } & 0.77 \\ \mathrm{~d}_{\text {min }} \text { used } & 0.82 \\ \text { Friedel pairs merged } & 0 \\ \text { Rint } & 0.0248 \\ \text { Intensity transformed } & 0 \\ \text { Omitted by user (OMIT hkl) } & 0 \\ \text { Maximum multiplicity } & 15 \\ & \\ \text { Filtered off (Shel/OMIT) } & 0\end{array}$

Table S-3: Fractional Atomic Coordinates $\left(\mathrm{x} 10^{4}\right)$ and Equivalent Isotropic Displacement Parameters $\left(\AA^{2} \times 10^{3}\right)$ for LDS224_130K. $U_{e q}$ is defined as $1 / 3$ of the trace of the orthogonalised $U_{i j}$. 


\begin{tabular}{|c|c|c|c|c|}
\hline Atom & $\mathbf{x}$ & $\mathbf{y}$ & $\mathbf{z}$ & $U_{e q}$ \\
\hline$\overline{01}$ & $-2436.8(7)$ & $-8287.3(17)$ & $-5173.6(11)$ & $26.4(3)$ \\
\hline N4 & $-3308.1(8)$ & $-6689(2)$ & $-3627.1(13)$ & $24.3(3)$ \\
\hline N6 & $-2932.1(9)$ & $-6708(2)$ & $-4260.7(14)$ & $27.0(3)$ \\
\hline N7 & $-3030.5(9)$ & $-9726(2)$ & $-4343.0(15)$ & $30.4(4)$ \\
\hline C10 & $-2782.8(9)$ & $-8267(2)$ & $-4616.2(15)$ & $23.4(3)$ \\
\hline $\mathrm{C} 1$ & $-4039.0(9)$ & $-6383(2)$ & $-2109.3(14)$ & $21.3(3)$ \\
\hline $\mathrm{C} 4$ & $-3895.1(9)$ & $-4929(2)$ & $-2801.0(16)$ & $23.8(4)$ \\
\hline C6 & $-3494.5(10)$ & $-5169(2)$ & $-3448.1(16)$ & $24.6(3)$ \\
\hline $\mathrm{C} 8$ & $-4308.0(9)$ & $-5276(2)$ & $-1381.8(15)$ & $21.4(3)$ \\
\hline C9 & $-3590.5(9)$ & $-4810(2)$ & $4.6(16)$ & $24.1(4)$ \\
\hline $\mathrm{C} 12$ & $-3280.9(9)$ & $-7451(2)$ & $-1092.1(15)$ & $23.8(3)$ \\
\hline C13 & $-4598.9(10)$ & $-3457(2)$ & $-2064.6(17)$ & $25.9(4)$ \\
\hline C16 & $-4700.4(10)$ & $-7645(2)$ & $-3113.8(17)$ & $27.7(4)$ \\
\hline C17 & $-3877.4(10)$ & $-3187(3)$ & $343.0(17)$ & $31.1(4)$ \\
\hline C19 & $-4195.3(10)$ & $-3389(2)$ & $-2768.8(17)$ & $27.2(4)$ \\
\hline $\mathrm{C} 22$ & $-5505.2(10)$ & $-3274(3)$ & $-3041.3(18)$ & $33.7(4)$ \\
\hline $\mathrm{C} 23$ & $-4259.2(11)$ & $-2098(2)$ & $-922.3(18)$ & $32.1(4)$ \\
\hline $\mathrm{C} 24$ & $-3251.5(10)$ & $-6413(3)$ & $907.0(15)$ & $27.3(4)$ \\
\hline $\mathrm{C} 27$ & $-3670.2(11)$ & $-6856(3)$ & $1568.3(18)$ & $35.6(4)$ \\
\hline C30 & $-3300.6(10)$ & $-7986(2)$ & $79.3(16)$ & $27.0(4)$ \\
\hline
\end{tabular}

Table S-4: Anisotropic Displacement Parameters (x104) LDS224_130K. The anisotropic displacement factor exponent takes the form: $-2 \pi^{2}\left[h^{2} a^{* 2} \times U_{11}+\ldots+2 h k a^{*} \times b^{*}{ }_{x} U_{12}\right]$

\begin{tabular}{lrrrrrr}
\hline Atom & $\boldsymbol{U}_{11}$ & $\boldsymbol{U}_{22}$ & $\boldsymbol{U}_{33}$ & $\boldsymbol{U}_{23}$ & $\boldsymbol{U}_{13}$ & $\boldsymbol{U}_{12}$ \\
\hline O1 & $30.1(6)$ & $29.5(6)$ & $29.0(6)$ & $-0.1(5)$ & $22.4(5)$ & $1.6(5)$ \\
$\mathrm{N} 4$ & $26.0(7)$ & $30.0(8)$ & $23.0(6)$ & $1.7(6)$ & $17.5(6)$ & $0.3(6)$ \\
$\mathrm{N} 6$ & $35.0(8)$ & $26.2(8)$ & $32.8(7)$ & $2.2(6)$ & $27.2(7)$ & $0.1(6)$ \\
$\mathrm{N} 7$ & $40.7(8)$ & $27.0(8)$ & $37.4(9)$ & $1.3(6)$ & $30.6(8)$ & $1.8(7)$ \\
C10 & $20.9(7)$ & $28.9(8)$ & $19.6(7)$ & $0.5(7)$ & $11.1(6)$ & $1.6(7)$ \\
C1 & $21.8(7)$ & $23.0(8)$ & $20.6(7)$ & $-0.2(6)$ & $13.0(6)$ & $-0.6(6)$ \\
C4 & $22.6(7)$ & $27.2(9)$ & $22.7(8)$ & $0.8(6)$ & $13.7(7)$ & $-1.3(7)$ \\
C6 & $27.4(8)$ & $26.4(8)$ & $25.0(8)$ & $2.2(7)$ & $18.0(7)$ & $-0.7(7)$ \\
C8 & $18.7(7)$ & $25.5(8)$ & $22.4(7)$ & $0.1(7)$ & $13.2(6)$ & $-0.4(6)$ \\
C9 & $20.5(7)$ & $28.8(9)$ & $24.0(8)$ & $-6.0(6)$ & $13.3(7)$ & $-3.0(6)$
\end{tabular}




\begin{tabular}{lrrrrrr}
\hline Atom & $\boldsymbol{U}_{11}$ & $\boldsymbol{U}_{22}$ & $\boldsymbol{U}_{33}$ & $\boldsymbol{U}_{23}$ & $\boldsymbol{U}_{13}$ & $\boldsymbol{U}_{12}$ \\
\hline C12 & $23.2(8)$ & $24.2(8)$ & $25.7(8)$ & $-1.3(7)$ & $15.0(7)$ & $2.3(7)$ \\
C13 & $26.0(8)$ & $26.6(9)$ & $29.7(8)$ & $2.0(7)$ & $18.6(7)$ & $2.5(7)$ \\
C16 & $28.7(8)$ & $28.6(9)$ & $25.3(8)$ & $-3.4(7)$ & $15.2(7)$ & $-4.6(7)$ \\
C17 & $31.5(9)$ & $33.2(9)$ & $31.7(8)$ & $-8.1(8)$ & $19.9(7)$ & $-1.3(8)$ \\
C19 & $29.3(8)$ & $26.2(9)$ & $31.2(8)$ & $3.7(7)$ & $20.3(7)$ & $0.7(7)$ \\
C22 & $28.6(9)$ & $41.5(10)$ & $33.5(9)$ & $10.9(9)$ & $19.3(8)$ & $11.9(9)$ \\
C23 & $36.4(9)$ & $25.6(9)$ & $42.4(10)$ & $-3.4(8)$ & $27.5(9)$ & $2.2(7)$ \\
C24 & $21.7(7)$ & $36.5(10)$ & $20.7(7)$ & $-1.8(7)$ & $10.4(6)$ & $1.5(7)$ \\
C27 & $35.1(9)$ & $48.3(12)$ & $25.9(8)$ & $6.4(8)$ & $18.8(8)$ & $7.0(9)$ \\
C30 & $25.8(8)$ & $29.0(9)$ & $25.1(8)$ & $4.4(7)$ & $13.9(7)$ & $4.6(7)$
\end{tabular}

Table S-5: Bond Lengths in Å for LDS224_130K.

\begin{tabular}{lll}
\hline Atom & Atom & Length/Å \\
\hline $\mathrm{O} 1$ & $\mathrm{C} 10$ & $1.242(2)$ \\
$\mathrm{N} 4$ & $\mathrm{~N} 6$ & $1.3808(18)$ \\
$\mathrm{N} 4$ & $\mathrm{C} 6$ & $1.276(2)$ \\
$\mathrm{N} 6$ & $\mathrm{C} 10$ & $1.360(2)$ \\
$\mathrm{N} 7$ & $\mathrm{C} 10$ & $1.343(2)$ \\
$\mathrm{C} 1$ & $\mathrm{C} 4$ & $1.529(2)$ \\
$\mathrm{C} 1$ & $\mathrm{C} 8$ & $1.549(2)$ \\
$\mathrm{C} 1$ & $\mathrm{C} 12$ & $1.555(2)$ \\
$\mathrm{C} 1$ & $\mathrm{C} 16$ & $1.541(2)$ \\
$\mathrm{C} 4$ & $\mathrm{C} 6$ & $1.452(2)$ \\
$\mathrm{C} 4$ & $\mathrm{C} 19$ & $1.334(3)$
\end{tabular}

\begin{tabular}{lll}
\hline Atom & Atom & Length/A \\
\hline C8 & C9 & $1.532(2)$ \\
C8 & C13 & $1.552(2)$ \\
C9 & C17 & $1.526(2)$ \\
C9 & C24 & $1.528(3)$ \\
C12 & C30 & $1.533(2)$ \\
C13 & C19 & $1.510(2)$ \\
C13 & C22 & $1.534(2)$ \\
C13 & C23 & $1.560(2)$ \\
C17 & C23 & $1.535(3)$ \\
C24 & C27 & $1.526(2)$ \\
C24 & C30 & $1.542(2)$
\end{tabular}

Table S-6: Bond Angles in ${ }^{\circ}$ for LDS224_130K.

\begin{tabular}{lllc}
\hline Atom & Atom & Atom & Angle ${ }^{\circ}$ \\
\hline C6 & N4 & N6 & $115.40(14)$ \\
C10 & N6 & N4 & $119.80(14)$ \\
01 & C10 & N6 & $119.87(16)$ \\
01 & C10 & N7 & $123.29(16)$ \\
N7 & C10 & N6 & $116.83(13)$
\end{tabular}

\begin{tabular}{lllc}
\hline Atom & Atom & Atom & Angle $^{\circ}$ \\
\hline C4 & C1 & C8 & $100.47(13)$ \\
C4 & C1 & C12 & $114.22(13)$ \\
C4 & C1 & C16 & $111.06(13)$ \\
C8 & C1 & C12 & $109.49(12)$ \\
C16 & C1 & C8 & $111.69(13)$
\end{tabular}




\begin{tabular}{|c|c|c|c|c|c|c|c|}
\hline Atom & Atom & Atom & Angle $/{ }^{\circ}$ & Atom & Atom & Atom & Angle $/^{\circ}$ \\
\hline$\overline{\mathrm{C} 16}$ & $\mathrm{C} 1$ & C12 & $109.65(14)$ & $\overline{\text { C19 }}$ & C13 & $\mathrm{C} 8$ & $100.44(13)$ \\
\hline C6 & $\mathrm{C} 4$ & $\mathrm{C} 1$ & $124.85(14)$ & C19 & C13 & $\mathrm{C} 22$ & $110.79(14)$ \\
\hline C19 & C4 & $\mathrm{C} 1$ & $112.22(14)$ & C19 & C13 & $\mathrm{C} 23$ & $113.39(14)$ \\
\hline C19 & $\mathrm{C} 4$ & C6 & $122.92(15)$ & C22 & C13 & $\mathrm{C} 8$ & $115.08(15)$ \\
\hline N4 & C6 & $\mathrm{C} 4$ & $122.05(15)$ & $\mathrm{C} 22$ & C13 & $\mathrm{C} 23$ & $111.43(14)$ \\
\hline $\mathrm{C} 1$ & C8 & $\mathrm{C} 13$ & $108.63(13)$ & C9 & C17 & $\mathrm{C} 23$ & $101.85(14)$ \\
\hline C9 & C8 & $\mathrm{C} 1$ & $110.40(12)$ & $\mathrm{C} 4$ & C19 & $\mathrm{C} 13$ & $113.67(15)$ \\
\hline C9 & C8 & C13 & $103.17(13)$ & C17 & $\mathrm{C} 23$ & C13 & $105.83(14)$ \\
\hline C17 & C9 & $\mathrm{C} 8$ & $102.95(13)$ & C9 & $\mathrm{C} 24$ & C30 & $107.58(13)$ \\
\hline $\mathrm{C} 17$ & C9 & $\mathrm{C} 24$ & $121.93(14)$ & $\mathrm{C} 27$ & $\mathrm{C} 24$ & C9 & $114.30(14)$ \\
\hline $\mathrm{C} 24$ & C9 & C8 & $112.28(14)$ & $\mathrm{C} 27$ & $\mathrm{C} 24$ & C30 & $110.19(15)$ \\
\hline C30 & C12 & $\mathrm{C} 1$ & $110.64(12)$ & C12 & C30 & $\mathrm{C} 24$ & $113.51(14)$ \\
\hline C8 & $\mathrm{C} 13$ & $\mathrm{C} 23$ & $105.24(13)$ & & & & \\
\hline
\end{tabular}

Table S-7: Hydrogen Fractional Atomic Coordinates $\left(\mathrm{x} 10^{4}\right)$ and Equivalent Isotropic Displacement Parameters $\left(\AA^{2} \times 10^{3}\right)$ for LDS224_130K. $U_{\text {eq }}$ is defined as $1 / 3$ of the trace of the orthogonalised $U_{i j}$.

\begin{tabular}{lccccc}
\hline Atom & & $\mathbf{x}$ & $\mathbf{y}$ & $\mathbf{z}$ & $\boldsymbol{U}_{\text {eq }}$ \\
\hline H6 & -2790 & -5715 & -4434 & 32 \\
H7A & -2950 & -10764 & -4561 & 36 \\
H7B & -3273 & -9646 & -3946 & 36 \\
H6A & -3367 & -4164 & -3748 & 30 \\
H8 & -4732 & -5892 & -1360 & 26 \\
H9 & -3163 & -4396 & -92 & 29 \\
H12A & -3250 & -8516 & -1518 & 29 \\
H12B & -2801 & -6730 & -778 & 29 \\
H16A & -5189 & -6978 & -3718 & 42 \\
H16B & -4803 & -8521 & -2649 & 42 \\
H16C & -4530 & -8240 & -3613 & 42 \\
H17A & -3427 & -2552 & 1107 & 37 \\
H17B & -4272 & -3501 & 530 & 37 \\
H19 & -4159 & -2347 & -3153 & 33 \\
H22A & -5708 & -4228 & -3687 & 51 \\
H22B & -5630 & -2141 & -3494 & 51 \\
H22C & -5756 & -3337 & -2566 & 51
\end{tabular}




\begin{tabular}{lrrrrr}
\hline Atom & & $\mathbf{x}$ & $\mathbf{y}$ & $\mathbf{z}$ & $\boldsymbol{U}_{\text {eq }}$ \\
\hline H23A & -3856 & -1337 & -890 & 38 \\
H23B & -4691 & -1349 & -1042 & 38 \\
H24 & -2677 & -6182 & 1610 & 33 \\
H27A & -3595 & -5890 & 2151 & 53 \\
H27B & -3443 & -7937 & 2083 & 53 \\
H27C & -4241 & -7025 & 895 & 53 \\
H30A & -2849 & -8789 & 652 & 32 \\
H30B & -3800 & -8640 & -251 & 32
\end{tabular}

Table S-8: Hydrogen Bond information for LDS224_130K.

\begin{tabular}{lllcccc}
\hline $\mathbf{D}$ & $\mathbf{H}$ & $\mathbf{A}$ & $\mathbf{d}(\mathbf{D}-\mathbf{H}) / \AA$ & $\mathbf{d}(\mathbf{H}-\mathbf{A}) / \AA$ & $\mathbf{d}(\mathbf{D}-\mathbf{A}) / \AA$ & $\mathbf{D}-\mathbf{H}-\mathbf{A} / \mathbf{d e g}$ \\
\hline N6 & H6 & $01^{1}$ & 0.88 & 2.03 & $2.9067(19)$ & 173.2 \\
N7 & H7A & $01^{2}$ & 0.88 & 2.17 & $3.046(2)$ & 171.1 \\
---- & & & & & \\
1-1/2-x,1/2+y,-1-z; ${ }^{2-1 / 2-x,-1 / 2+y,-1-z}$ & & & &
\end{tabular}




\section{LDS221_130K}

Submitted by: Leonidas Dimitrios Syntrivanis, University of Basel

Solved by: Alessandro Prescimone

Sample ID: $\quad$ LDS221_130K

Crystal Data and Experimental
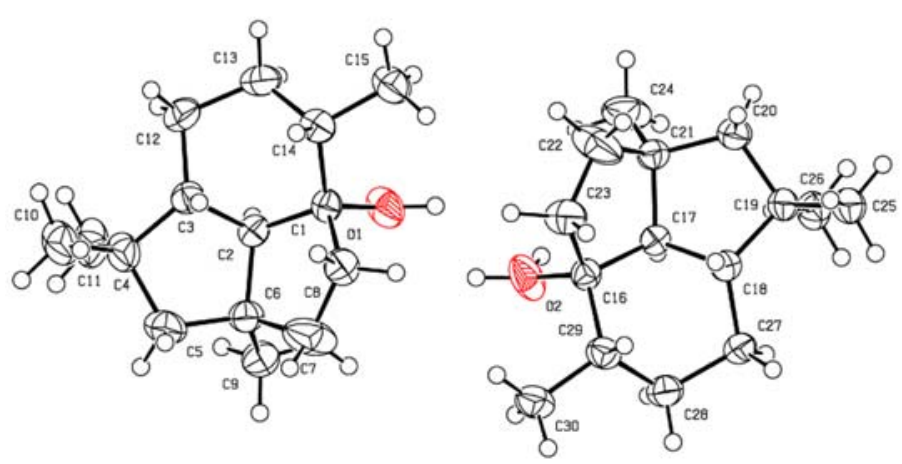

Experimental. Single colourless block-shaped crystals of LDS221_130K were recrystallised from pentane by slow evaporation. A suitable crystal $0.23 \times 0.22 \times 0.18 \mathrm{~mm}^{3}$ was selected and mounted on a mylar loop in perfluoroether oil on an Bruker APEX-II CCD diffractometer. The crystal was kept at a steady $T=130 \mathrm{~K}$ during data collection. The structure was solved with the ShelXT (Sheldrick, 2015) structure solution program using the Intrinsic Phasing solution method and by using Olex2 (Dolomanov et al., 2009 ) as the graphical interface. The model was refined with version 2014/7 of ShelXL (Sheldrick, 2015) using Least Squares minimisation.

Crystal Data. $\mathrm{C}_{15} \mathrm{H}_{26} \mathrm{O}, M_{r}=222.36$, tetragonal, $P 4_{3}{ }_{2} 2$ (No. 96) $, \mathrm{a}=16.2888(11) \AA, \quad \mathrm{b}=16.2888(11) \AA, \quad \mathrm{c}=$ 20.5020(15) $\AA, \alpha=\beta=\gamma=90^{\circ}, V=5439.7(8) \AA^{3}, T=130 \mathrm{~K}$, $Z=16, Z^{\prime}=2, \mu\left(\mathrm{CuK}_{\alpha}\right)=0.491,37629$ reflections measured, 5077 unique $\left(R_{\text {int }}=0.0426\right)$ which were used in all calculations. The final $w R_{2}$ was 0.1419 (all data) and $R_{1}$ was $0.0538(\mathrm{I}>2(\mathrm{I}))$.

\section{Compound}

LDS221_130K

\begin{tabular}{|c|c|}
\hline Formula & $\mathrm{C}_{15} \mathrm{H}_{26} \mathrm{O}$ \\
\hline$D_{\text {calc. }} / \mathrm{g} \mathrm{cm}^{-3}$ & 1.086 \\
\hline$\mu / \mathrm{mm}^{-1}$ & 0.491 \\
\hline Formula Weight & 222.36 \\
\hline Colour & colourless \\
\hline Shape & block \\
\hline Size $/ \mathrm{mm}^{3}$ & $0.23 \times 0.22 \times 0.18$ \\
\hline$T / \mathrm{K}$ & 130 \\
\hline Crystal System & tetragonal \\
\hline Flack Parameter & $0.10(5)$ \\
\hline Hooft Parameter & $0.10(6)$ \\
\hline Space Group & $P 4_{3} 2_{1} 2$ \\
\hline$a / \AA ̊$ & $16.2888(11)$ \\
\hline$b / \AA$ & $16.2888(11)$ \\
\hline$c / \AA ̊$ & $20.5020(15)$ \\
\hline$\alpha /^{\circ}$ & 90 \\
\hline$\beta /^{\circ}$ & 90 \\
\hline$\gamma /^{\circ}$ & 90 \\
\hline $\mathrm{V} / \AA^{3}$ & $5439.7(8)$ \\
\hline$Z$ & 16 \\
\hline$Z^{\prime}$ & 2 \\
\hline Wavelength/Å & 1.541838 \\
\hline Radiation type & $\mathrm{CuK}_{\alpha}$ \\
\hline$\Theta_{\min } /^{\circ}$ & 3.465 \\
\hline$\Theta_{\max } /^{o}$ & 70.226 \\
\hline Measured Refl. & 37629 \\
\hline Independent Refl. & 5077 \\
\hline $\begin{array}{l}\text { Reflections with I > } \\
2(\text { I) }\end{array}$ & 4913 \\
\hline$R_{\text {int }}$ & 0.0426 \\
\hline Parameters & 309 \\
\hline Restraints & 10 \\
\hline Largest Peak & 0.527 \\
\hline Deepest Hole & -0.350 \\
\hline GooF & 1.064 \\
\hline$w R_{2}$ (all data) & 0.1419 \\
\hline$w R_{2}$ & 0.1403 \\
\hline$R_{1}$ (all data) & 0.0553 \\
\hline$R_{1}$ & 0.0538 \\
\hline
\end{tabular}


Structure Quality Indicators

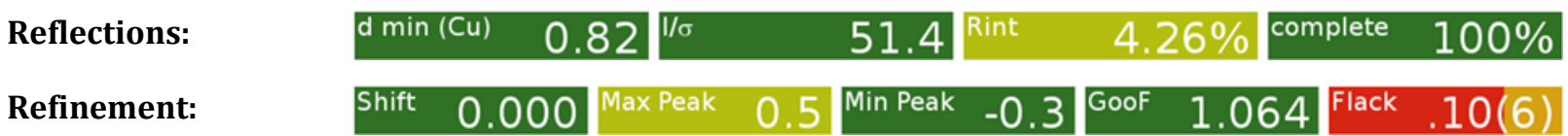

A colourless block-shaped crystal with dimensions $0.23 \times 0.22 \times 0.18 \mathrm{~mm}^{3}$ was mounted on a mylar loop in perfluoroether oil. Data were collected using an Bruker APEX-II CCD diffractometer equipped with an Oxford Cryosystems low-temperature device operating at $T=130 \mathrm{~K}$.

Data were measured using $\phi \& \omega$ scans using $\mathrm{CuK}_{\alpha}$ radiation. The total number of runs and images was based on the strategy calculation from the program APEX2 (Bruker, V2, n/a) The maximum resolution that was achieved was $\Theta=70.226^{\circ}(0.82 \AA)$.

The diffraction pattern was indexed. The total number of runs and images was based on the strategy calculation from the program APEX2 (Bruker, V2, n/a) and the unit cell was refined using SAINT (Bruker, V8.34A, after 2013) on 9093 reflections, $24 \%$ of the observed reflections.

Data reduction, scaling and absorption corrections were performed using SAINT (Bruker, V8.34A, after 2013). The final completeness is $100.00 \%$ out to $70.226^{\circ}$ in $\Theta$. A multi-scan absorption correction was performed using SADABS-2012/1 (Bruker,2012) was used for absorption correction. $w R_{2}$ (int) was 0.0871 before and 0.0545 after correction. The Ratio of minimum to maximum transmission is 0.8735 .The $\lambda / 2$ correction factor is 0.0015 .. The absorption coefficient $\mu$ of this material is $0.491 \mathrm{~mm}^{-1}$ at this wavelength $(\lambda=1.542 \AA)$ and the minimum and maximum transmissions are 0.658 and 0.753 .

The structure was solved and the space group $P 4_{3} 2{ }_{1} 2$ (\# 96) determined by the ShelXT (Sheldrick, 2015) structure solution program using Intrinsic Phasing and refined by Least Squares using version 2014/7 of ShelXL (Sheldrick, 2015). All non-hydrogen atoms were refined anisotropically. Hydrogen atom positions were calculated geometrically and refined using the riding model. Most hydrogen atom positions were calculated geometrically and refined using the riding model, but some hydrogen atoms were refined freely.

_exptl_absorpt_process_details: SADABS-2012/1 (Bruker,2012) was used for absorption correction. $w R_{2}$ (int) was 0.0871 before and 0.0545 after correction. The Ratio of minimum to maximum transmission is 0.8735 . The $\lambda / 2$ correction factor is 0.0015 .

The value of $Z^{\prime}$ is 2 . This means that there are two independent molecules in the asymmetric unit.

The Flack parameter was refined to 0.10(5). Determination of absolute structure using Bayesian statistics on Bijvoet differences using the Olex2 results in $0.10(6)$. Note: The Flack parameter is used to determine chirality of the crystal studied, the value should be near 0 , a value of 1 means that the stereochemistry is wrong and the model should be inverted. A value of 0.5 means that the crystal consists of a racemic mixture of the two enantiomers. 

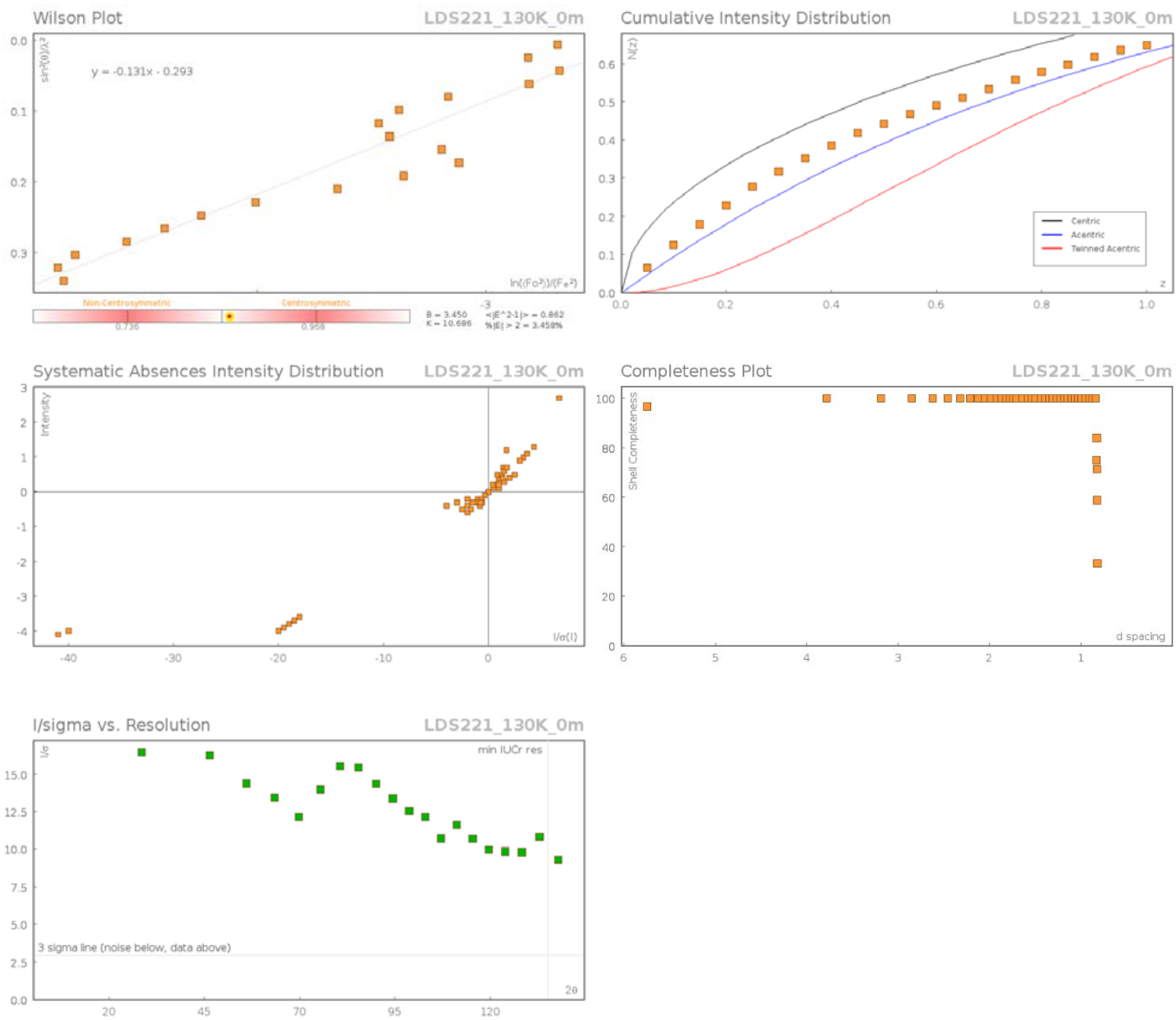

\section{Data Plots: Refinement and Data}
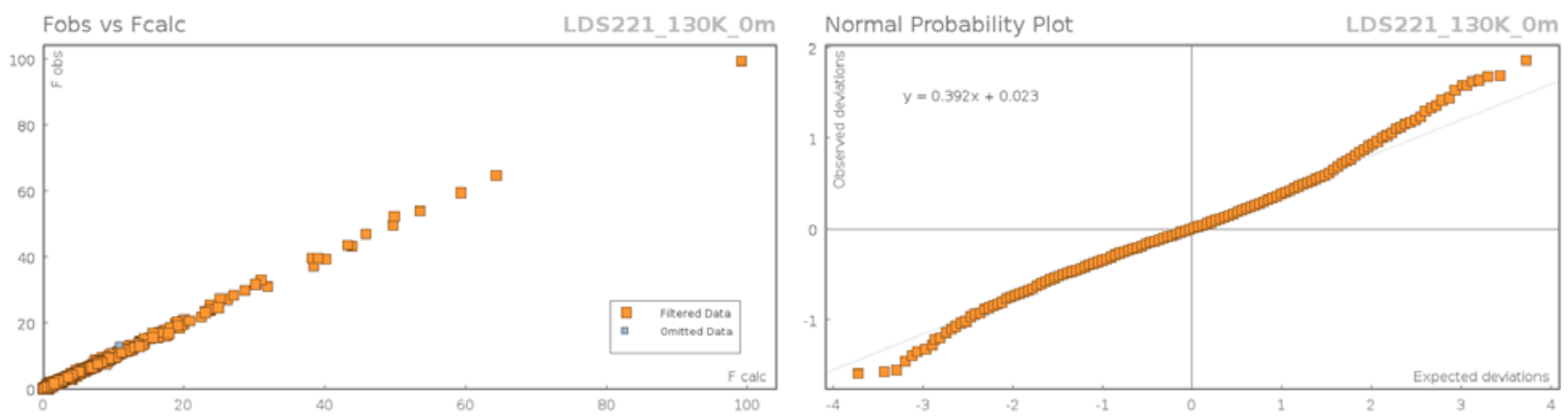

\section{Reflection Statistics}

Total reflections (after filtering)

Completeness

$\mathrm{hkl}_{\max }$ collected

$\mathrm{hkl}_{\max }$ used

Lim $\mathrm{d}_{\max }$ collected

$\mathrm{d}_{\max }$ used
37756

$(19,19,15)$

$(13,19,24)$

100.0

20.5
Unique reflections

Mean I/ $\sigma$

$\mathrm{hkl}_{\text {min }}$ collected

$\mathrm{hkl}_{\min }$ used

Lim $d_{\text {min }}$ collected

$\mathrm{d}_{\min }$ used

S53
5077

33.78

$(-19,-18,-24)$

$(-13,0,0)$

0.77

0.82 


$\begin{array}{llll}\text { Friedel pairs } & 4177 & \text { Friedel pairs merged } & 0 \\ \text { Inconsistent equivalents } & 0 & \mathrm{R}_{\text {int }} & 0.0426 \\ \mathrm{R}_{\text {sigma }} & 0.0195 & \text { Intensity transformed } & 0 \\ \text { Omitted reflections } & 0 & \text { Omitted by user (OMIT hkl) } & 0 \\ \text { Multiplicity } & (11337,6193,2390,1076, & \text { Maximum multiplicity } & 31 \\ & 243,101,94,10) & & \\ \text { Removed systematic absences } 127 & \text { Filtered off (Shel/OMIT) } & 0\end{array}$

Table S-9: Fractional Atomic Coordinates $\left(\mathrm{x} 10^{4}\right)$ and Equivalent Isotropic Displacement Parameters $\left(\AA^{2} \times 10^{3}\right)$ for LDS221_130K. $U_{e q}$ is defined as $1 / 3$ of the trace of the orthogonalised $U_{i j}$.

\begin{tabular}{lllcc}
\hline Atom & \multicolumn{1}{c}{$\mathbf{x}$} & \multicolumn{1}{c}{$\mathbf{y}$} & $\mathbf{Z}$ & $\boldsymbol{U}_{\text {eq }}$ \\
\hline O1 & $4493.8(15)$ & $5319.2(16)$ & $3177.9(12)$ & $53.5(6)$ \\
O2 & $5792.8(14)$ & $4159.1(18)$ & $3189.1(13)$ & $61.0(7)$ \\
C17 & $7268.4(16)$ & $4179.3(16)$ & $3328.5(12)$ & $28.5(5)$ \\
C18 & $7948.8(17)$ & $3703.0(17)$ & $3663.9(13)$ & $31.0(6)$ \\
C16 & $6438.5(16)$ & $4024.8(17)$ & $3652.6(13)$ & $31.6(6)$ \\
C1 & $3884.7(17)$ & $5514.8(17)$ & $3659.6(12)$ & $31.7(6)$ \\
C2 & $3031.5(17)$ & $5460.0(17)$ & $3360.7(12)$ & $31.1(6)$ \\
C8 & $3875(2)$ & $4828(2)$ & $4173.2(15)$ & $43.1(7)$ \\
C19 & $8734.8(17)$ & $4180.4(18)$ & $3497.5(14)$ & $35.3(6)$ \\
C4 & $1587(2)$ & $5621(2)$ & $3599.3(17)$ & $48.4(8)$ \\
C3 & $2431.9(18)$ & $6010.5(17)$ & $3722.6(14)$ & $35.7(6)$ \\
C21 & $7499.8(18)$ & $5091.9(17)$ & $3416.7(14)$ & $34.0(6)$ \\
C26 & $9026.1(19)$ & $4021(2)$ & $2797.9(15)$ & $44.2(7)$ \\
C20 & $8426.9(19)$ & $5076.2(18)$ & $3576.0(15)$ & $39.3(7)$ \\
C14 & $4028.0(19)$ & $6388.5(18)$ & $3952.4(14)$ & $38.2(6)$ \\
C29 & $6399.6(18)$ & $3139.9(18)$ & $3937.5(15)$ & $38.6(7)$ \\
C6 & $2701.2(19)$ & $4576.8(18)$ & $3442.6(14)$ & $38.6(7)$ \\
C23 & $6399(2)$ & $4695(2)$ & $4177.4(16)$ & $49.1(8)$ \\
C13 & $3552(2)$ & $7035.0(19)$ & $3565.3(17)$ & $46.8(8)$ \\
C25 & $9435.7(19)$ & $3980(2)$ & $3962.1(17)$ & $45.3(7)$ \\
C27 & $7854.5(18)$ & $2784.0(17)$ & $3534.5(15)$ & $37.6(6)$ \\
C28 & $6932(2)$ & $2554.9(18)$ & $3535.1(16)$ & $42.6(7)$ \\
C15 & $4936(2)$ & $6608(2)$ & $4020.0(17)$ & $48.9(8)$ \\
C30 & $5526(2)$ & $2826(2)$ & $4022.4(16)$ & $45.7(7)$ \\
C12 & $2614(2)$ & $6905.5(19)$ & $3577.9(17)$ & $45.6(7)$ \\
\hline & $1260(2)$ & $5807(3)$ & $2918.7(19)$ & $64.3(11)$ \\
\hline
\end{tabular}




\begin{tabular}{lcccc}
\hline Atom & $\mathbf{x}$ & $\mathbf{y}$ & $\mathbf{z}$ & $\boldsymbol{U}_{\text {eq }}$ \\
\hline C24 & $7326(3)$ & $5604(2)$ & $2813(2)$ & $73.2(13)$ \\
C5 & $1815(2)$ & $4702(2)$ & $3688(2)$ & $57.2(10)$ \\
C10 & $956(2)$ & $5911(3)$ & $4102(2)$ & $67.0(11)$ \\
C7 & $3324(3)$ & $4178(3)$ & $3920(2)$ & $75.2(14)$ \\
C22 & $6946(3)$ & $5363(3)$ & $3975(3)$ & $82.1(16)$
\end{tabular}

Table S-10: Anisotropic Displacement Parameters (x104) LDS221_130K. The anisotropic displacement factor exponent takes the form: $-2 \pi^{2}\left[h^{2} a^{* 2} \times U_{11}+\ldots+2 h k a^{*}{ }_{x} b^{*}{ }_{x} U_{12}\right]$

\begin{tabular}{|c|c|c|c|c|c|c|}
\hline Atom & $U_{11}$ & $U_{22}$ & $\boldsymbol{U}_{33}$ & $U_{23}$ & $U_{13}$ & $U_{12}$ \\
\hline$\overline{01}$ & $42.7(13)$ & $60.8(15)$ & $57.0(13)$ & $-21.3(12)$ & $21.7(12)$ & $-8.8(11)$ \\
\hline 02 & $34.5(12)$ & $78.3(18)$ & $70.0(16)$ & $37.5(14)$ & $-17.3(12)$ & $-10.5(12)$ \\
\hline C17 & $31.5(13)$ & $29.7(13)$ & $24.4(11)$ & $0.5(10)$ & $-1.9(10)$ & $1.2(11)$ \\
\hline C18 & $33.2(14)$ & $31.5(13)$ & $28.3(12)$ & $0.1(11)$ & $-4.4(11)$ & $0.3(11)$ \\
\hline $\mathrm{C} 16$ & $30.8(13)$ & $32.7(14)$ & $31.3(12)$ & $2.2(11)$ & $-1.4(11)$ & $0.9(11)$ \\
\hline $\mathrm{C} 1$ & $31.7(14)$ & $36.0(14)$ & $27.5(12)$ & $-0.8(11)$ & $5.1(11)$ & $2.0(11)$ \\
\hline $\mathrm{C} 2$ & $34.7(14)$ & $32.5(13)$ & $26.1(12)$ & $-0.7(10)$ & $2.2(10)$ & $3.0(11)$ \\
\hline $\mathrm{C} 8$ & $41.5(17)$ & $46.4(17)$ & $41.5(15)$ & $12.3(13)$ & $-4.8(13)$ & $-0.4(13)$ \\
\hline C19 & $31.9(14)$ & $34.8(14)$ & $39.2(14)$ & $2.4(12)$ & $-5.4(12)$ & $-0.2(12)$ \\
\hline $\mathrm{C} 4$ & $36.1(16)$ & $52.2(19)$ & $56.9(19)$ & $-19.1(16)$ & $6.2(15)$ & $-0.5(14)$ \\
\hline C3 & $37.0(15)$ & $33.9(15)$ & $36.2(14)$ & $-8.6(12)$ & $1.0(11)$ & $2.5(12)$ \\
\hline $\mathrm{C} 21$ & $35.2(15)$ & $29.1(13)$ & $37.8(14)$ & $1.9(11)$ & $2.6(12)$ & $0.1(11)$ \\
\hline C26 & $37.1(15)$ & $49.2(18)$ & $46.2(16)$ & $1.0(14)$ & $5.0(13)$ & $1.4(14)$ \\
\hline $\mathrm{C} 20$ & $38.8(16)$ & $33.9(15)$ & $45.2(16)$ & $0.5(13)$ & $-5.8(13)$ & $-2.6(12)$ \\
\hline C14 & $40.3(15)$ & $40.2(15)$ & $34.0(13)$ & $-4.0(12)$ & $3.4(12)$ & $-4.3(12)$ \\
\hline $\mathrm{C} 29$ & $38.7(15)$ & $36.1(15)$ & $41.2(15)$ & $5.5(12)$ & $-1.6(13)$ & $-5.2(12)$ \\
\hline $\mathrm{C} 6$ & $41.3(16)$ & $33.3(14)$ & $41.2(15)$ & $-0.4(12)$ & $-3.1(13)$ & $-1.3(12)$ \\
\hline $\mathrm{C} 23$ & $59(2)$ & $41.5(17)$ & $46.4(17)$ & $-7.3(14)$ & $22.3(16)$ & $-4.6(15)$ \\
\hline C13 & $57(2)$ & $30.7(15)$ & $52.9(18)$ & $-1.0(14)$ & $4.8(16)$ & $-4.0(14)$ \\
\hline $\mathrm{C} 25$ & $37.4(16)$ & $42.9(17)$ & $55.5(18)$ & $3.7(15)$ & $-11.8(14)$ & $-1.0(13)$ \\
\hline $\mathrm{C} 27$ & $37.2(15)$ & $30.1(14)$ & $45.5(15)$ & $1.2(12)$ & $-4.5(12)$ & $2.0(11)$ \\
\hline $\mathrm{C} 28$ & $45.8(17)$ & $32.0(14)$ & $50.0(17)$ & $-0.7(13)$ & $-5.6(14)$ & $-1.2(13)$ \\
\hline C15 & $43.9(17)$ & $51.6(19)$ & $51.1(18)$ & $-9.1(16)$ & $3.8(15)$ & $-9.7(14)$ \\
\hline C30 & $48.5(18)$ & $40.1(16)$ & $48.6(17)$ & $6.6(14)$ & $0.0(14)$ & $-9.4(14)$ \\
\hline C12 & $49.2(18)$ & $33.5(15)$ & $54.0(18)$ & $-6.3(14)$ & $0.0(15)$ & $6.5(13)$ \\
\hline
\end{tabular}




\begin{tabular}{lllcccc}
\hline Atom & \multicolumn{1}{c}{$\boldsymbol{U}_{11}$} & $\boldsymbol{U}_{22}$ & $\boldsymbol{U}_{33}$ & $\boldsymbol{U}_{23}$ & $\boldsymbol{U}_{13}$ & $\boldsymbol{U}_{12}$ \\
\hline C9 & $48.2(19)$ & $41.4(18)$ & $78(2)$ & $-24.3(17)$ & $7.6(17)$ & $0.3(15)$ \\
C11 & $43.9(19)$ & $78(3)$ & $71(2)$ & $-29(2)$ & $-16.7(18)$ & $11.7(19)$ \\
C24 & $75(3)$ & $45(2)$ & $99(3)$ & $33(2)$ & $-44(2)$ & $-17.8(18)$ \\
C5 & $56(2)$ & $43.2(18)$ & $73(2)$ & $-16.1(17)$ & $22.6(19)$ & $-12.7(16)$ \\
C10 & $42.1(19)$ & $70(2)$ & $89(3)$ & $-32(2)$ & $20.0(19)$ & $-4.8(18)$ \\
C7 & $86(3)$ & $59(2)$ & $81(3)$ & $34(2)$ & $-36(2)$ & $-28(2)$ \\
C22 & $71(3)$ & $68(3)$ & $108(3)$ & $-54(3)$ & $52(3)$ & $-35(2)$
\end{tabular}

Table S-11: Bond Lengths in Å for LDS221_130K.

\begin{tabular}{|c|c|c|c|c|c|}
\hline Atom & Atom & Length/Å & Atom & Atom & Length/Å \\
\hline$\overline{01}$ & C1 & $1.436(3)$ & $\overline{\mathrm{C} 4}$ & $\mathrm{C} 3$ & $1.537(4)$ \\
\hline 02 & $\mathrm{C} 16$ & $1.434(3)$ & $\mathrm{C} 4$ & $\mathrm{C} 11$ & $1.524(5)$ \\
\hline $\mathrm{C} 17$ & C18 & $1.518(4)$ & $\mathrm{C} 4$ & $\mathrm{C} 5$ & $1.552(5)$ \\
\hline $\mathrm{C} 17$ & C16 & $1.527(4)$ & $\mathrm{C} 4$ & C10 & $1.529(5)$ \\
\hline $\mathrm{C} 17$ & $\mathrm{C} 21$ & $1.544(4)$ & $\mathrm{C} 3$ & $\mathrm{C} 12$ & $1.517(4)$ \\
\hline C18 & C19 & $1.536(4)$ & $\mathrm{C} 21$ & $\mathrm{C} 20$ & $1.545(4)$ \\
\hline C18 & $\mathrm{C} 27$ & $1.528(4)$ & $\mathrm{C} 21$ & $\mathrm{C} 24$ & $1.519(4)$ \\
\hline C16 & $\mathrm{C} 29$ & $1.557(4)$ & $\mathrm{C} 21$ & $\mathrm{C} 22$ & $1.522(4)$ \\
\hline C16 & $\mathrm{C} 23$ & $1.534(4)$ & C14 & $\mathrm{C} 13$ & $1.529(4)$ \\
\hline $\mathrm{C} 1$ & $\mathrm{C} 2$ & $1.521(4)$ & C14 & C15 & $1.528(4)$ \\
\hline $\mathrm{C} 1$ & $\mathrm{C} 8$ & $1.537(4)$ & $\mathrm{C} 29$ & $\mathrm{C} 28$ & $1.530(4)$ \\
\hline $\mathrm{C} 1$ & $\mathrm{C} 14$ & $1.562(4)$ & $\mathrm{C} 29$ & $\mathrm{C} 30$ & $1.523(4)$ \\
\hline $\mathrm{C} 2$ & $\mathrm{C} 3$ & $1.519(4)$ & $\mathrm{C} 6$ & $\mathrm{C} 9$ & $1.519(4)$ \\
\hline $\mathrm{C} 2$ & C6 & $1.545(4)$ & $\mathrm{C} 6$ & $\mathrm{C} 5$ & $1.541(5)$ \\
\hline $\mathrm{C} 8$ & $\mathrm{C} 7$ & $1.482(5)$ & $\mathrm{C} 6$ & $\mathrm{C} 7$ & $1.552(5)$ \\
\hline C19 & $\mathrm{C} 26$ & $1.533(4)$ & $\mathrm{C} 23$ & $\mathrm{C} 22$ & $1.466(5)$ \\
\hline C19 & $\mathrm{C} 20$ & $1.551(4)$ & C13 & C12 & $1.543(5)$ \\
\hline C19 & $\mathrm{C} 25$ & $1.522(4)$ & $\mathrm{C} 27$ & $\mathrm{C} 28$ & $1.548(4)$ \\
\hline
\end{tabular}

Table S-12: Bond Angles in ${ }^{\circ}$ for LDS221_130K.

\begin{tabular}{llll}
\hline Atom & Atom & Atom & Angle $^{\text {o }}$ \\
\hline C18 & C17 & C16 & $111.4(2)$
\end{tabular}

\begin{tabular}{llll}
\hline Atom & Atom & Atom & Angle $^{\text {o }}$ \\
\hline C18 & C17 & C21 & $105.1(2)$
\end{tabular}




\begin{tabular}{|c|c|c|c|c|c|c|c|}
\hline$\overline{\text { Atom }}$ & Atom & Atom & Angle/ ${ }^{\circ}$ & Atom & Atom & Atom & Angle $/^{\circ}$ \\
\hline$\overline{\mathrm{C} 16}$ & C17 & $\mathrm{C} 21$ & $108.9(2)$ & $\overline{\mathrm{C} 10}$ & $\mathrm{C} 4$ & $\mathrm{C} 5$ & $112.4(3)$ \\
\hline C17 & C18 & C19 & $104.4(2)$ & $\mathrm{C} 2$ & $\mathrm{C} 3$ & $\mathrm{C} 4$ & $104.6(2)$ \\
\hline C17 & C18 & $\mathrm{C} 27$ & $110.4(2)$ & $\mathrm{C} 12$ & C3 & $\mathrm{C} 2$ & $110.2(2)$ \\
\hline $\mathrm{C} 27$ & C18 & C19 & $122.8(2)$ & $\mathrm{C} 12$ & C3 & $\mathrm{C} 4$ & $122.7(3)$ \\
\hline 02 & C16 & C17 & $109.6(2)$ & $\mathrm{C} 17$ & $\mathrm{C} 21$ & $\mathrm{C} 20$ & $104.3(2)$ \\
\hline 02 & C16 & C29 & $111.1(2)$ & $\mathrm{C} 24$ & $\mathrm{C} 21$ & C17 & $112.8(3)$ \\
\hline 02 & C16 & $\mathrm{C} 23$ & $109.0(3)$ & $\mathrm{C} 24$ & $\mathrm{C} 21$ & $\mathrm{C} 20$ & $111.3(3)$ \\
\hline C17 & C16 & C29 & $110.6(2)$ & $\mathrm{C} 24$ & $\mathrm{C} 21$ & $\mathrm{C} 22$ & $110.1(4)$ \\
\hline C17 & C16 & $\mathrm{C} 23$ & $103.0(2)$ & $\mathrm{C} 22$ & $\mathrm{C} 21$ & C17 & $102.9(2)$ \\
\hline $\mathrm{C} 23$ & C16 & C29 & $113.2(2)$ & $\mathrm{C} 22$ & $\mathrm{C} 21$ & $\mathrm{C} 20$ & 115.1(3) \\
\hline 01 & $\mathrm{C} 1$ & $\mathrm{C} 2$ & $110.0(2)$ & $\mathrm{C} 21$ & $\mathrm{C} 20$ & C19 & $108.0(2)$ \\
\hline 01 & $\mathrm{C} 1$ & C8 & $108.5(3)$ & C13 & C14 & $\mathrm{C} 1$ & $110.6(2)$ \\
\hline 01 & $\mathrm{C} 1$ & C14 & $111.3(2)$ & C15 & C14 & $\mathrm{C} 1$ & 113.1(3) \\
\hline $\mathrm{C} 2$ & $\mathrm{C} 1$ & C8 & $102.9(2)$ & C15 & C14 & $\mathrm{C} 13$ & 112.1(3) \\
\hline $\mathrm{C} 2$ & $\mathrm{C} 1$ & C14 & $110.2(2)$ & $\mathrm{C} 28$ & $\mathrm{C} 29$ & C16 & $110.6(2)$ \\
\hline C8 & $\mathrm{C} 1$ & C14 & $113.7(2)$ & C30 & $\mathrm{C} 29$ & C16 & $113.1(2)$ \\
\hline $\mathrm{C} 1$ & $\mathrm{C} 2$ & C6 & $109.2(2)$ & C30 & $\mathrm{C} 29$ & $\mathrm{C} 28$ & $112.5(3)$ \\
\hline C3 & $\mathrm{C} 2$ & $\mathrm{C} 1$ & $110.8(2)$ & $\mathrm{C} 2$ & C6 & $\mathrm{C} 7$ & $103.3(2)$ \\
\hline C3 & $\mathrm{C} 2$ & C6 & $105.8(2)$ & C9 & C6 & $\mathrm{C} 2$ & $113.6(3)$ \\
\hline $\mathrm{C} 7$ & $\mathrm{C} 8$ & $\mathrm{C} 1$ & $106.6(3)$ & $\mathrm{C} 9$ & $\mathrm{C} 6$ & C5 & $111.2(3)$ \\
\hline C18 & C19 & $\mathrm{C} 20$ & $100.6(2)$ & C9 & C6 & $\mathrm{C} 7$ & $107.3(3)$ \\
\hline C26 & C19 & C18 & $112.3(2)$ & $\mathrm{C} 5$ & C6 & $\mathrm{C} 2$ & $103.8(2)$ \\
\hline C26 & C19 & $\mathrm{C} 20$ & $110.9(2)$ & $\mathrm{C} 5$ & C6 & $\mathrm{C} 7$ & $117.5(3)$ \\
\hline $\mathrm{C} 25$ & C19 & C18 & $112.2(2)$ & $\mathrm{C} 22$ & $\mathrm{C} 23$ & C16 & $107.7(3)$ \\
\hline $\mathrm{C} 25$ & C19 & $\mathrm{C} 26$ & $108.5(3)$ & C14 & $\mathrm{C} 13$ & C12 & $113.5(3)$ \\
\hline C25 & C19 & $\mathrm{C} 20$ & $112.3(2)$ & C18 & $\mathrm{C} 27$ & $\mathrm{C} 28$ & $109.5(2)$ \\
\hline C3 & $\mathrm{C} 4$ & C5 & $99.4(3)$ & $\mathrm{C} 29$ & $\mathrm{C} 28$ & $\mathrm{C} 27$ & $113.6(2)$ \\
\hline C11 & $\mathrm{C} 4$ & C3 & $112.4(3)$ & $\mathrm{C} 3$ & C12 & $\mathrm{C} 13$ & $109.2(3)$ \\
\hline C11 & $\mathrm{C} 4$ & $\mathrm{C} 5$ & $112.5(3)$ & $\mathrm{C} 6$ & $\mathrm{C} 5$ & $\mathrm{C} 4$ & $108.3(3)$ \\
\hline C11 & $\mathrm{C} 4$ & C10 & $108.7(3)$ & $\mathrm{C} 8$ & $\mathrm{C} 7$ & C6 & $108.5(3)$ \\
\hline C10 & $\mathrm{C} 4$ & C3 & $111.3(3)$ & $\mathrm{C} 23$ & $\mathrm{C} 22$ & $\mathrm{C} 21$ & $111.0(3)$ \\
\hline
\end{tabular}

Table S-13: Hydrogen Fractional Atomic Coordinates $\left(\mathrm{x} 10^{4}\right)$ and Equivalent Isotropic Displacement Parameters $\left(\AA^{2} \times 10^{3}\right)$ for LDS221_130K. $U_{e q}$ is defined as $1 / 3$ of the trace of the orthogonalised $U_{i j}$. 


\begin{tabular}{|c|c|c|c|c|}
\hline Atom & & & & $U_{e q}$ \\
\hline H17 & 7244 & 4035 & 2855 & 34 \\
\hline H18 & 7860 & 3779 & 4143 & 37 \\
\hline $\mathrm{H} 2$ & 3051 & 5612 & 2888 & 37 \\
\hline H8A & 4435 & 4608 & 4240 & 52 \\
\hline H8B & 3668 & 5040 & 4595 & 52 \\
\hline H3 & 2549 & 5931 & 4197 & 43 \\
\hline $\mathrm{H} 26 \mathrm{~A}$ & 9184 & 3443 & 2752 & 66 \\
\hline $\mathrm{H} 26 \mathrm{~B}$ & 9500 & 4371 & 2702 & 66 \\
\hline $\mathrm{H} 26 \mathrm{C}$ & 8581 & 4146 & 2492 & 66 \\
\hline $\mathrm{H} 20 \mathrm{~A}$ & 8521 & 5268 & 4028 & 47 \\
\hline H20B & 8729 & 5444 & 3275 & 47 \\
\hline H14 & 3792 & 6383 & 4403 & 46 \\
\hline H29 & 6648 & 3165 & 4383 & 46 \\
\hline $\mathrm{H} 23 \mathrm{~A}$ & 5830 & 4901 & 4222 & 59 \\
\hline H23B & 6577 & 4472 & 4604 & 59 \\
\hline $\mathrm{H} 13 \mathrm{~A}$ & 3742 & 7025 & 3107 & 56 \\
\hline H13B & 3679 & 7584 & 3746 & 56 \\
\hline $\mathrm{H} 25 \mathrm{~A}$ & 9266 & 4099 & 4411 & 68 \\
\hline H25B & 9915 & 4316 & 3851 & 68 \\
\hline $\mathrm{H} 25 \mathrm{C}$ & 9578 & 3398 & 3923 & 68 \\
\hline $\mathrm{H} 27 \mathrm{~A}$ & 8145 & 2468 & 3876 & 45 \\
\hline H27B & 8101 & 2644 & 3107 & 45 \\
\hline H28A & 6730 & 2552 & 3080 & 51 \\
\hline H28B & 6870 & 1992 & 3710 & 51 \\
\hline H15A & 5217 & 6180 & 4271 & 73 \\
\hline H15B & 4989 & 7136 & 4246 & 73 \\
\hline $\mathrm{H} 15 \mathrm{C}$ & 5185 & 6649 & 3586 & 73 \\
\hline H30A & 5205 & 3227 & 4271 & 69 \\
\hline Н30B & 5535 & 2302 & 4258 & 69 \\
\hline H30C & 5274 & 2746 & 3593 & 69 \\
\hline $\mathrm{H} 12 \mathrm{~A}$ & 2365 & 7258 & 3918 & 55 \\
\hline $\mathrm{H} 12 \mathrm{~B}$ & 2376 & 7059 & 3151 & 55 \\
\hline H9A & 2334 & 4318 & 2501 & 84 \\
\hline H9B & 2553 & 3511 & 2911 & 84 \\
\hline
\end{tabular}




\begin{tabular}{|c|c|c|c|c|}
\hline Atom & $\mathbf{x}$ & $\mathbf{y}$ & $\mathbf{z}$ & $U_{e q}$ \\
\hline$\overline{\mathrm{H} 9 \mathrm{C}}$ & 3273 & 4082 & 2634 & 84 \\
\hline $\mathrm{H} 11 \mathrm{~A}$ & 1121 & 6391 & 2888 & 96 \\
\hline $\mathrm{H} 11 \mathrm{~B}$ & 768 & 5476 & 2837 & 96 \\
\hline $\mathrm{H} 11 \mathrm{C}$ & 1681 & 5673 & 2594 & 96 \\
\hline $\mathrm{H} 24 \mathrm{~A}$ & 7646 & 5391 & 2446 & 110 \\
\hline H24B & 7480 & 6176 & 2896 & 110 \\
\hline $\mathrm{H} 24 \mathrm{C}$ & 6740 & 5575 & 2709 & 110 \\
\hline $\mathrm{H} 5 \mathrm{~A}$ & 1775 & 4547 & 4153 & 69 \\
\hline H5B & 1433 & 4353 & 3435 & 69 \\
\hline $\mathrm{H} 10 \mathrm{~A}$ & 1155 & 5785 & 4542 & 100 \\
\hline H10B & 433 & 5630 & 4027 & 100 \\
\hline $\mathrm{H} 10 \mathrm{C}$ & 877 & 6505 & 4058 & 100 \\
\hline H7A & 3028 & 3912 & 4285 & 90 \\
\hline H7B & 3649 & 3753 & 3690 & 90 \\
\hline $\mathrm{H} 22 \mathrm{~A}$ & 7288 & 5535 & 4350 & 99 \\
\hline $\mathrm{H} 22 \mathrm{~B}$ & 6615 & 5840 & 3835 & 99 \\
\hline $\mathrm{H} 2 \mathrm{~A}$ & $5254(17)$ & $4310(50)$ & $3210(40)$ & 50 \\
\hline $\mathrm{H} 2 \mathrm{~B}$ & $6000(40)$ & $4340(40)$ & $2802(18)$ & 50 \\
\hline $\mathrm{H} 1 \mathrm{~B}$ & $5032(17)$ & $5190(40)$ & $3260(40)$ & 50 \\
\hline $\mathrm{H} 1 \mathrm{~A}$ & $4410(40)$ & $5320(50)$ & $2738(11)$ & 50 \\
\hline
\end{tabular}

Table S-14: Hydrogen Bond information for LDS221_130K.

\begin{tabular}{lllrrrc}
\hline $\mathbf{D}$ & $\mathbf{H}$ & $\mathbf{A}$ & $\mathbf{d}(\mathbf{D}-\mathbf{H}) / \AA$ & $\mathbf{d}(\mathbf{H}-\mathbf{A}) / \AA$ & $\mathbf{d}(\mathbf{D}-\mathbf{A}) / \AA$ & $\mathbf{D}-\mathbf{H}-\mathbf{A} / \mathbf{d e g}$ \\
\hline $\mathrm{O} 2$ & $\mathrm{H} 2 \mathrm{~A}$ & $\mathrm{O} 1$ & $0.914(19)$ & $2.06(5)$ & $2.837(4)$ & $143(7)$ \\
02 & $\mathrm{H} 2 \mathrm{~B}$ & $02^{1}$ & $0.911(19)$ & $2.06(5)$ & $2.828(5)$ & $141(6)$ \\
01 & $\mathrm{H} 1 \mathrm{~B}$ & $\mathrm{O} 2$ & $0.914(19)$ & $2.09(5)$ & $2.837(4)$ & $138(6)$ \\
01 & $\mathrm{H} 1 \mathrm{~A}$ & $011^{1}$ & $0.912(19)$ & $1.95(3)$ & $2.813(5)$ & $156(7)$
\end{tabular}

$11-y, 1-x, 1 / 2-z$

Table S-15: Atomic Occupancies for all atoms that are not fully occupied in LDS221_130K.

\begin{tabular}{lrrrr}
\hline Atom & Occupancy & & $\overline{\text { Atom }}$ & Occupancy \\
\cline { 4 - 5 } & 0.5 & & H1B & 0.5 \\
H2A & 0.5 & & H1A & 0.5
\end{tabular}




\section{Citations}

O.V. Dolomanov and L.J. Bourhis and R.J. Gildea and J.A.K. Howard and H. Puschmann, Olex2: A complete structure solution, refinement and analysis program, J. Appl. Cryst., (2009), 42, 339-341.

Sheldrick, G.M., Crystal structure refinement with ShelXL, Acta Cryst., (2015), C27, 3-8.

Sheldrick, G.M., ShelXT-Integrated space-group and crystal-structure determination, Acta Cryst., (2015), A71, 3-8.

Software for the Integration of CCD Detector System Bruker Analytical X-ray Systems, Bruker axs, Madison, WI (after 2013). 


\section{Appendix C: NMR spectra of substrates}

${ }^{1} \mathrm{H}$ NMR (500 MHz, toluene-d8)

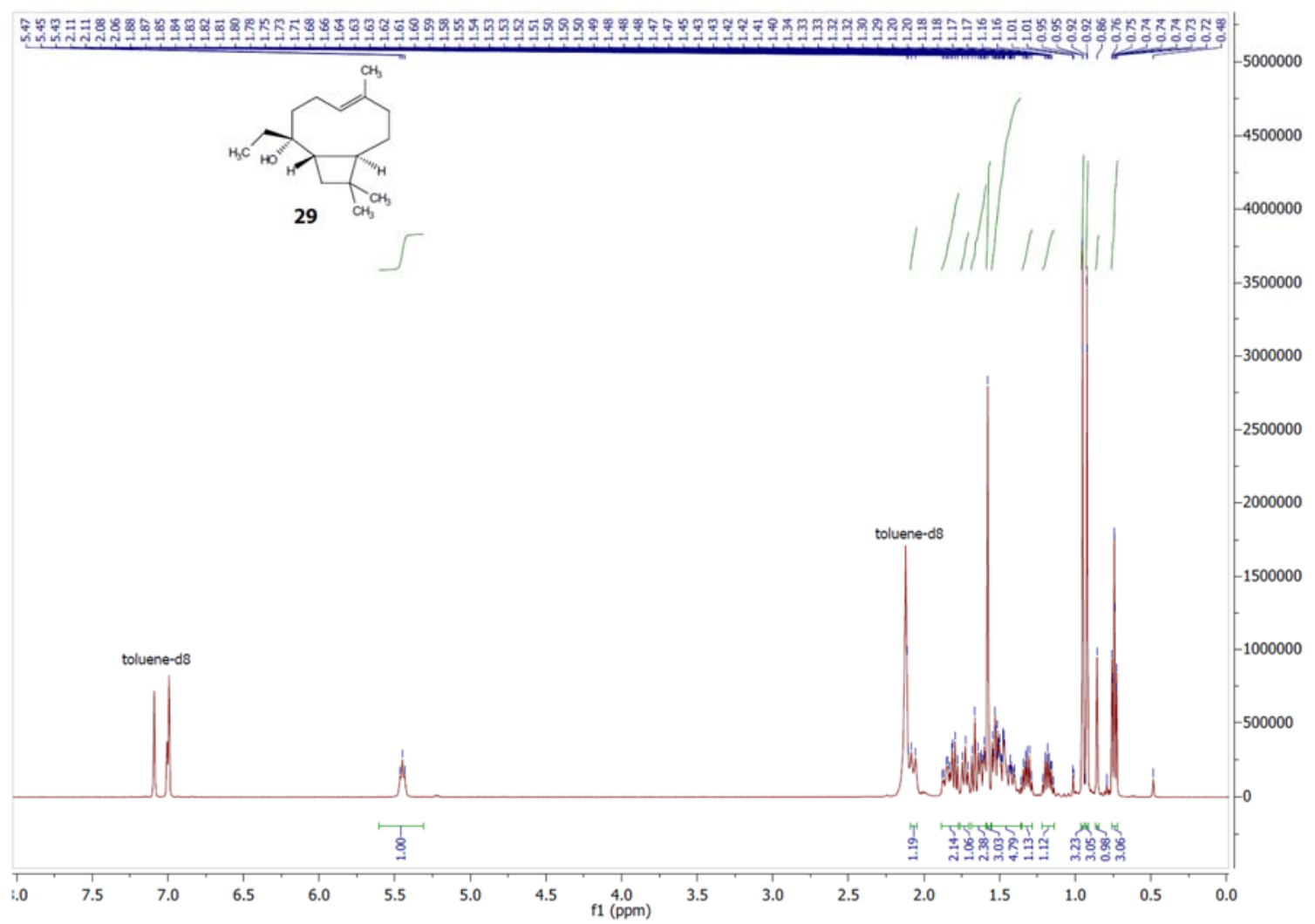

${ }^{13} \mathrm{C}$ NMR (126 MHz, toluene-d8)

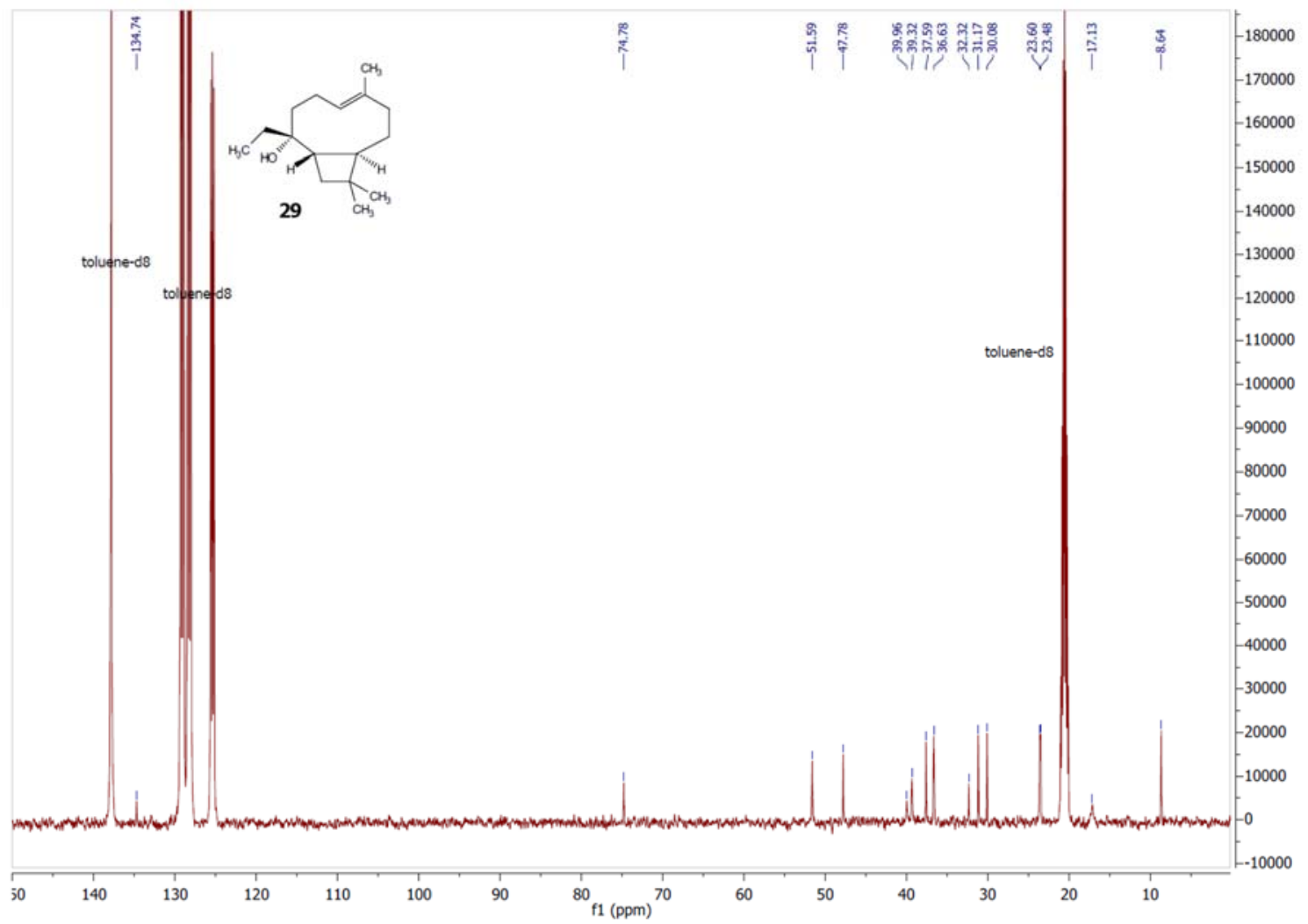




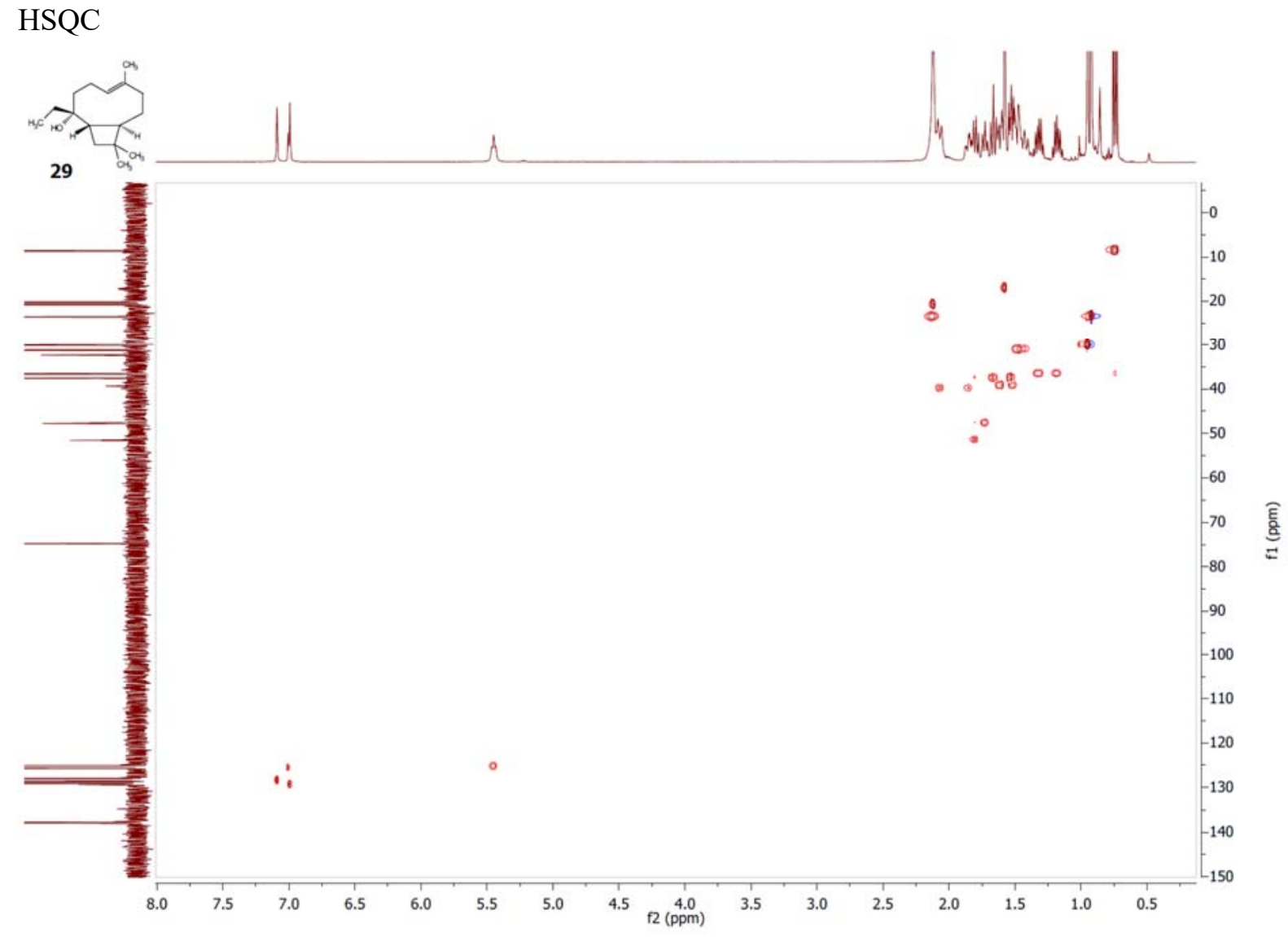

${ }^{1} \mathrm{H}$ NMR (500 MHz, toluene-d8)

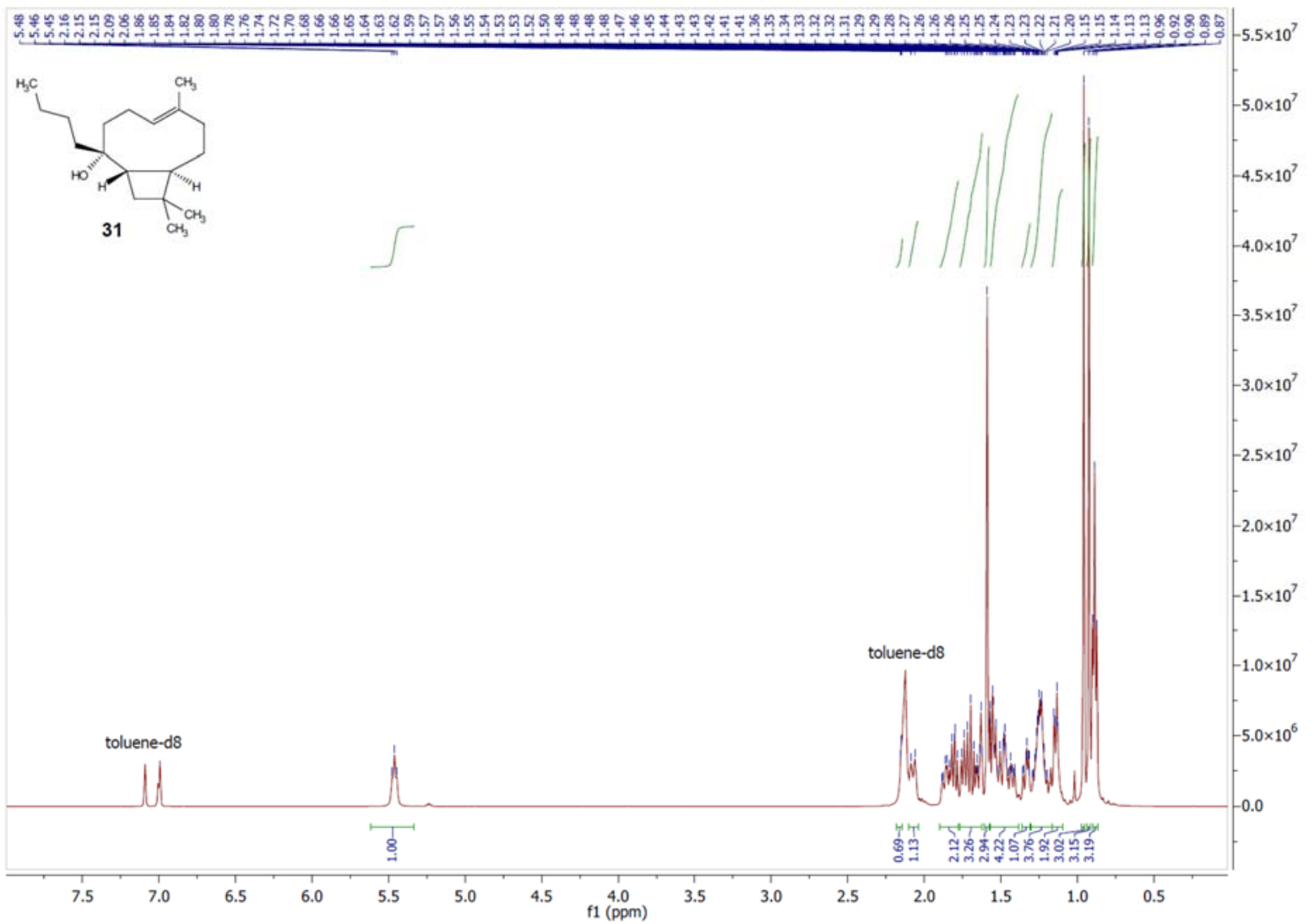


${ }^{13} \mathrm{C}$ NMR (126 MHz, toluene-d8)

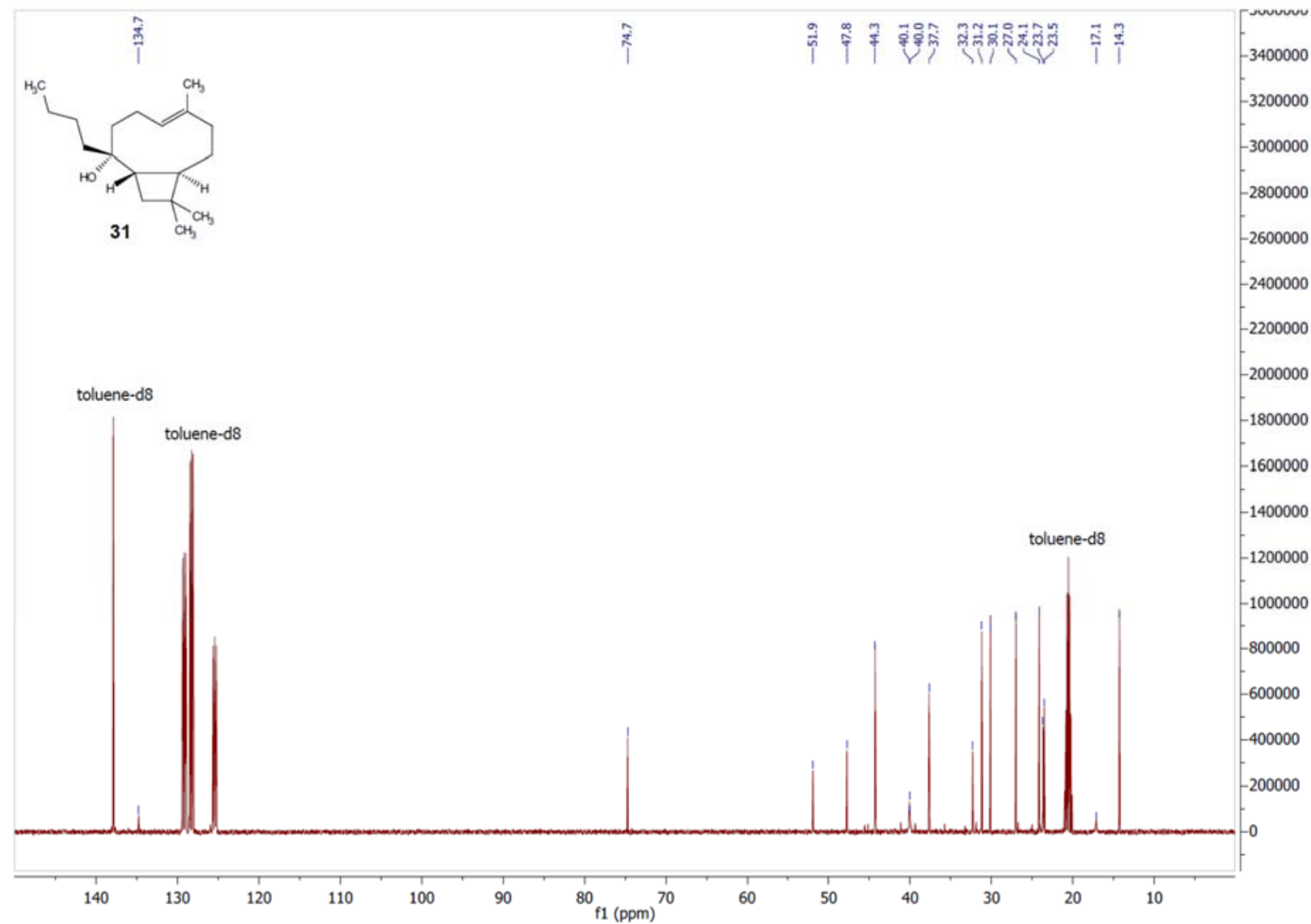

HSQC

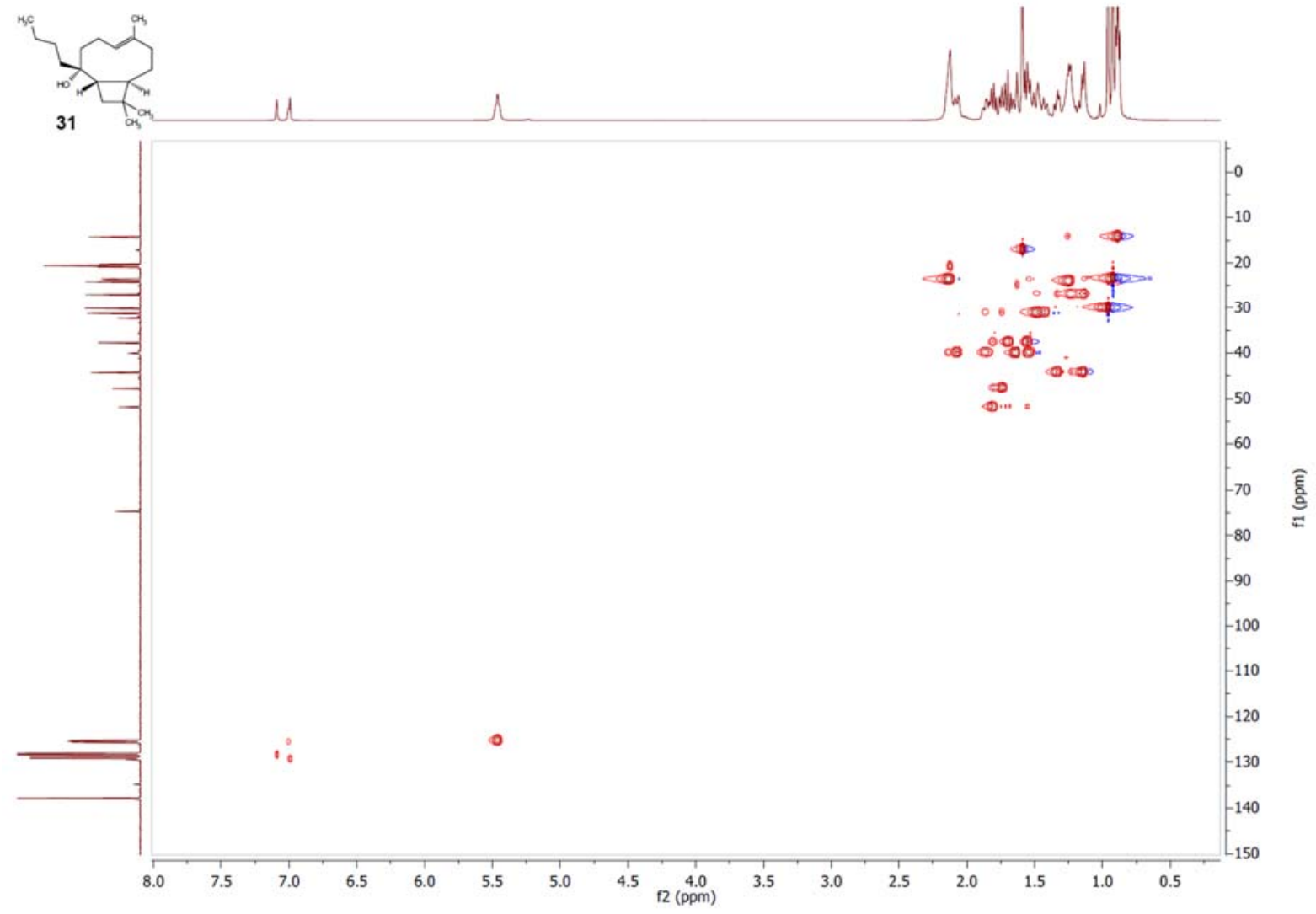


${ }^{1} \mathrm{H}$ NMR (500 MHz, toluene-d8)

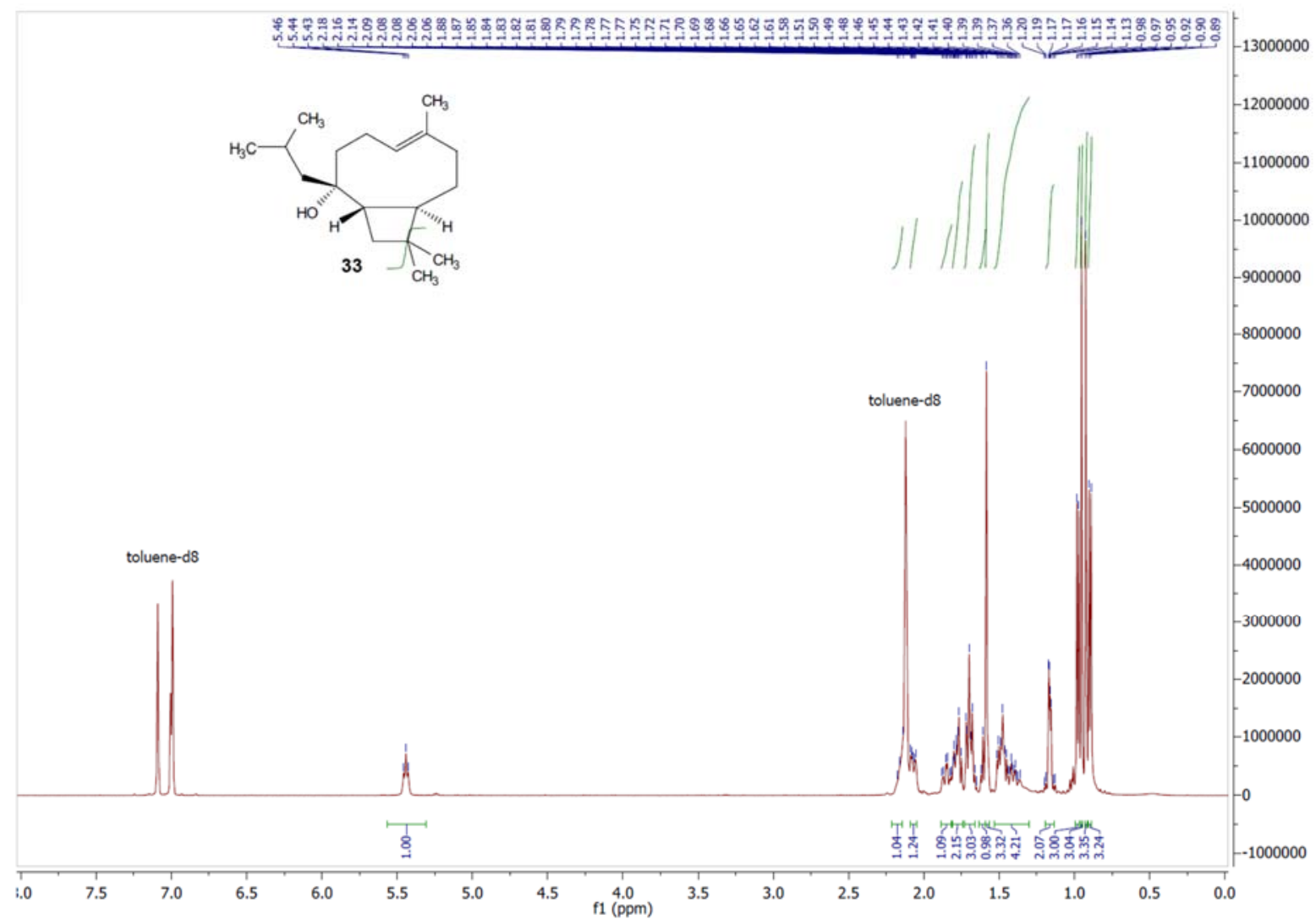

${ }^{13} \mathrm{C}$ NMR (126 MHz, toluene-d8)

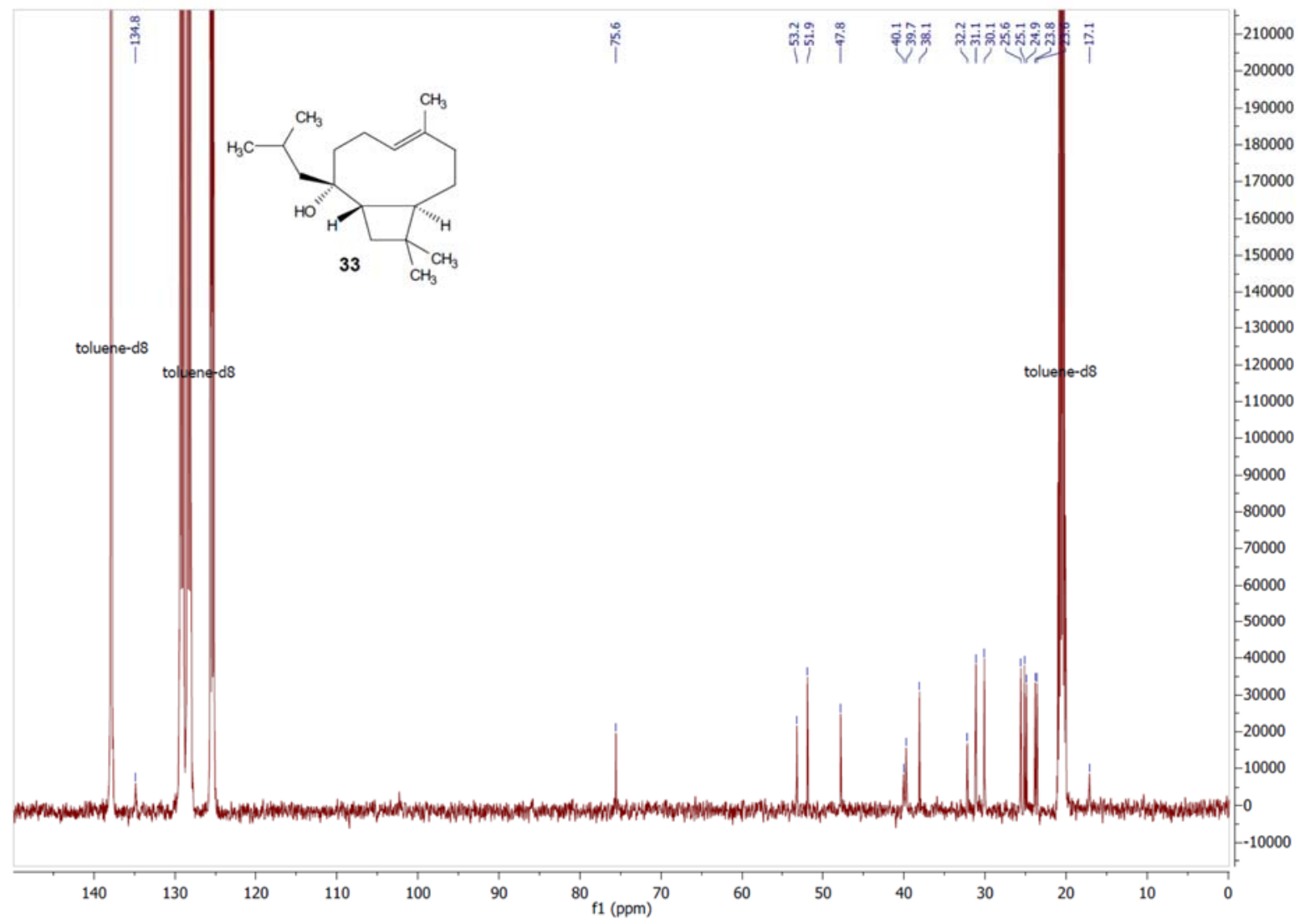


HSQC

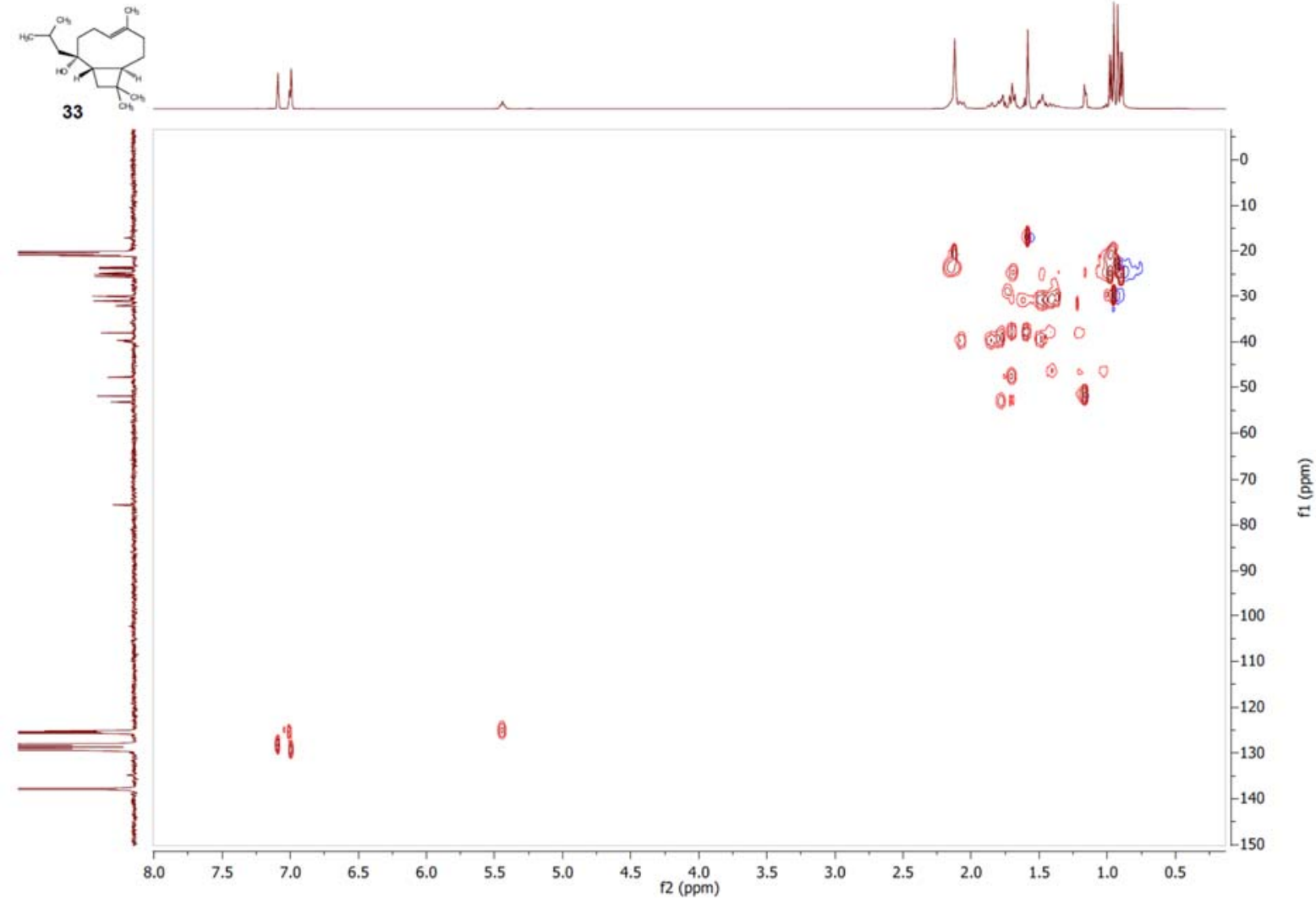

${ }^{1} \mathrm{H}$ NMR (500 MHz, toluene-d8)

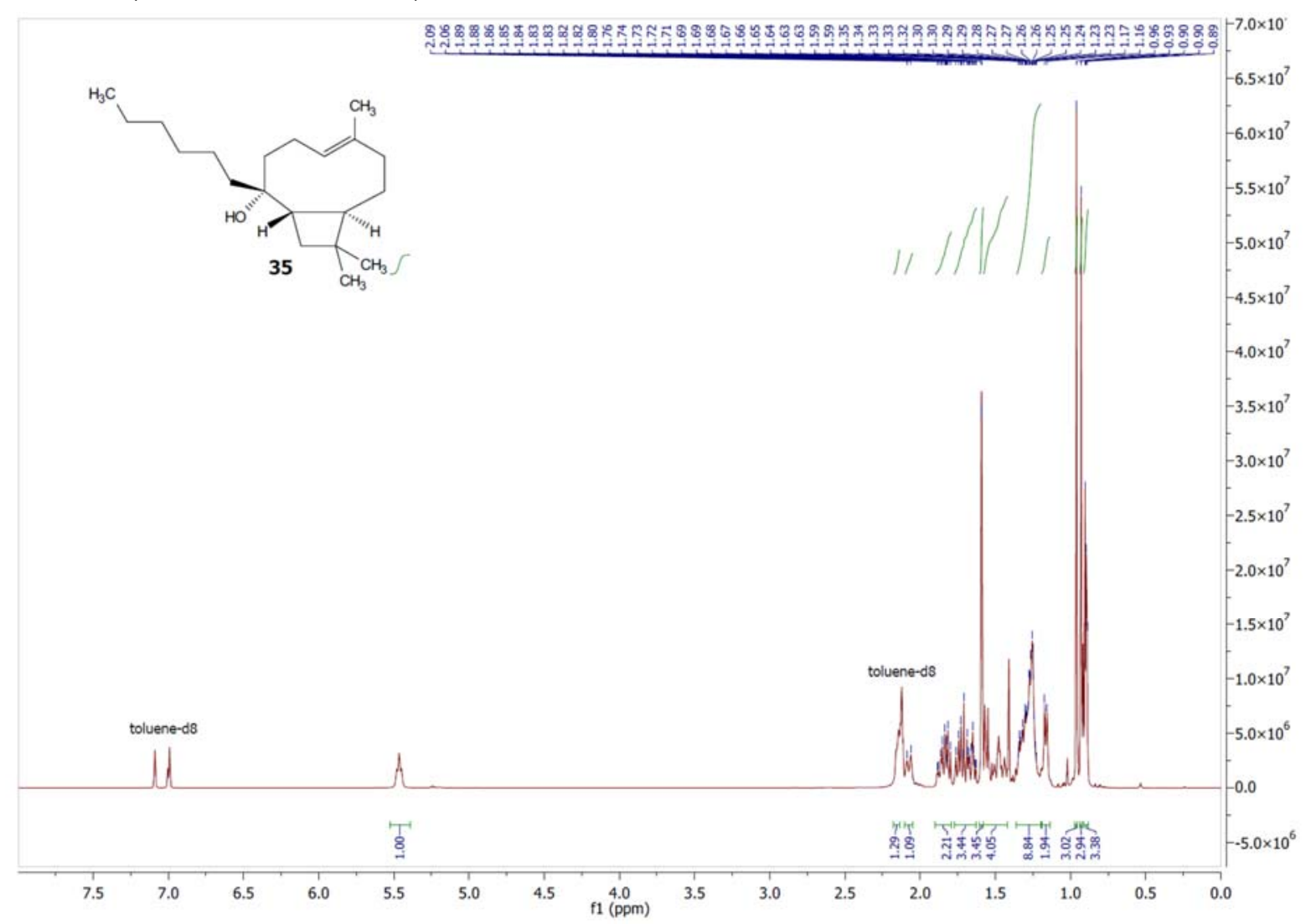


${ }^{13} \mathrm{C}$ NMR (126 MHz, toluene-d8)

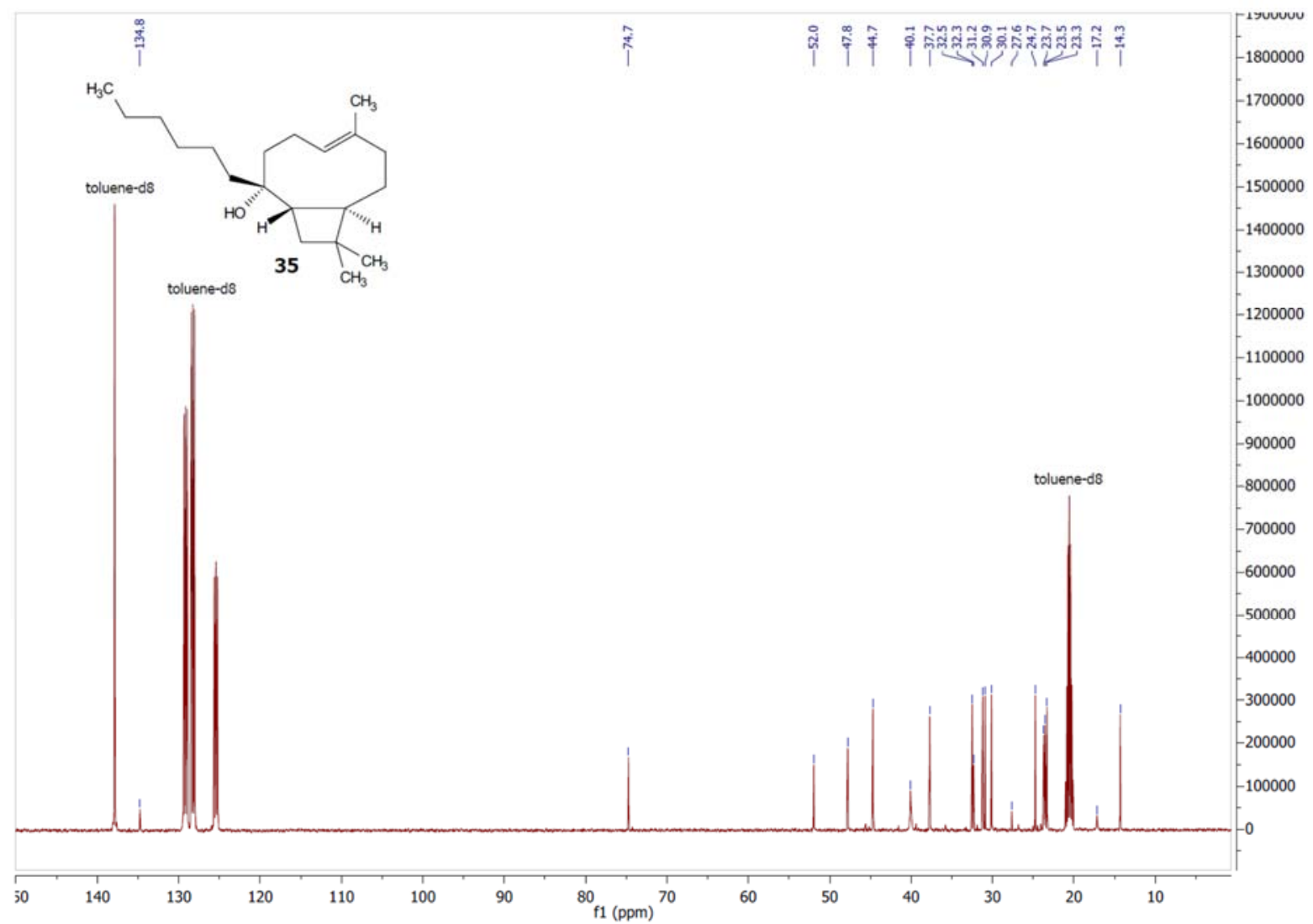

HSQC

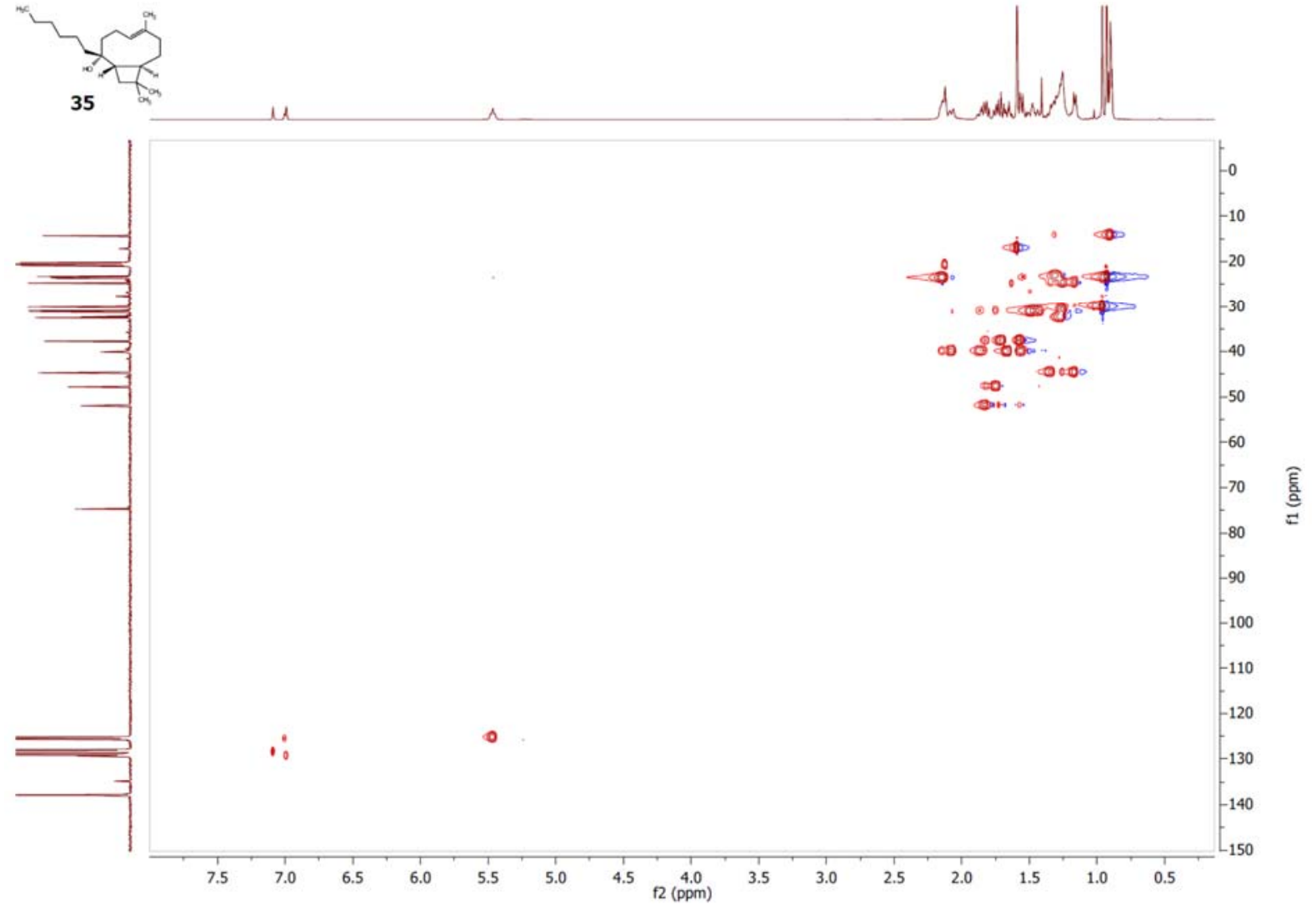


${ }^{1} \mathrm{H}$ NMR (500 MHz, toluene-d8)

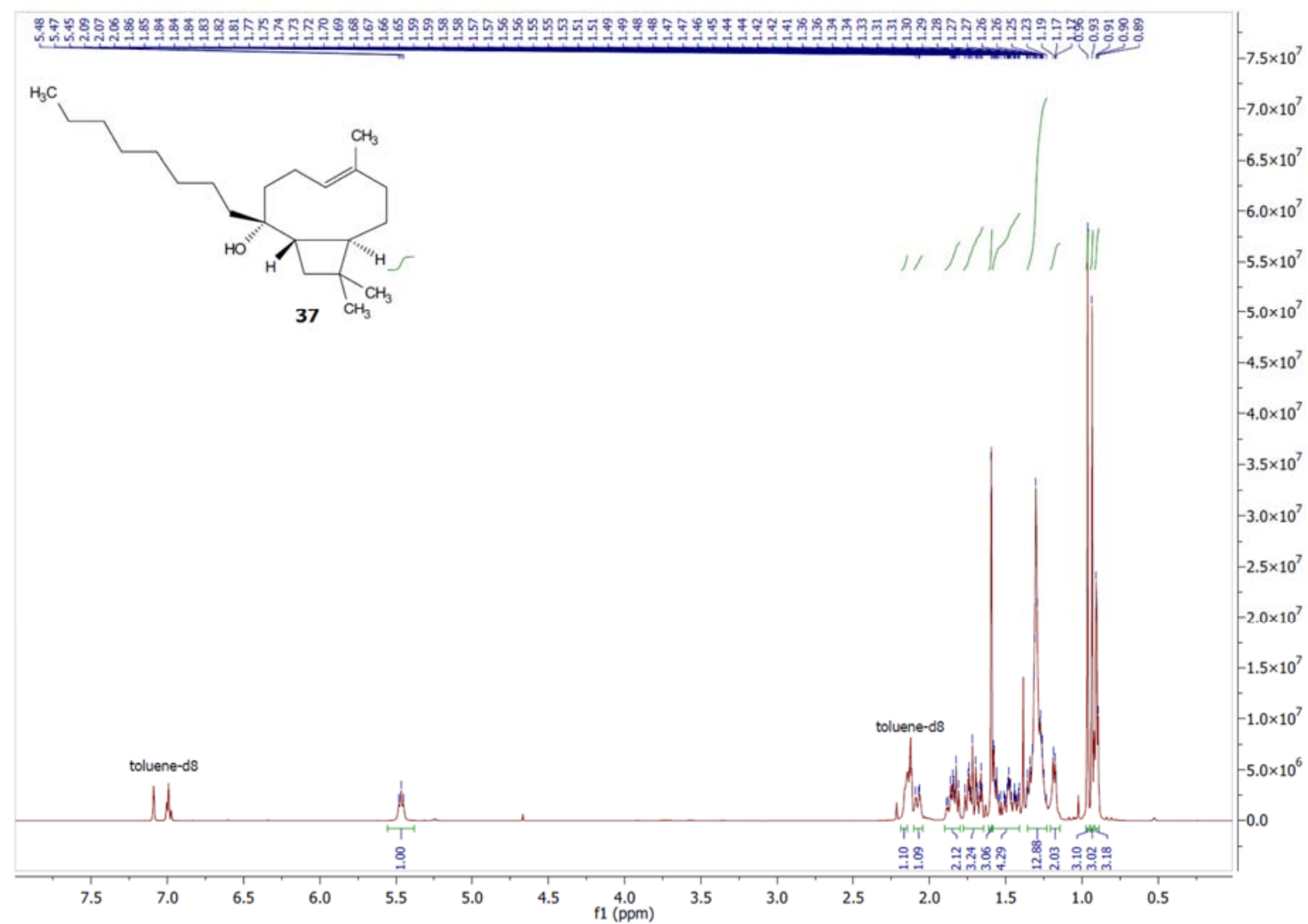

${ }^{13} \mathrm{C}$ NMR (126 MHz, toluene-d8)

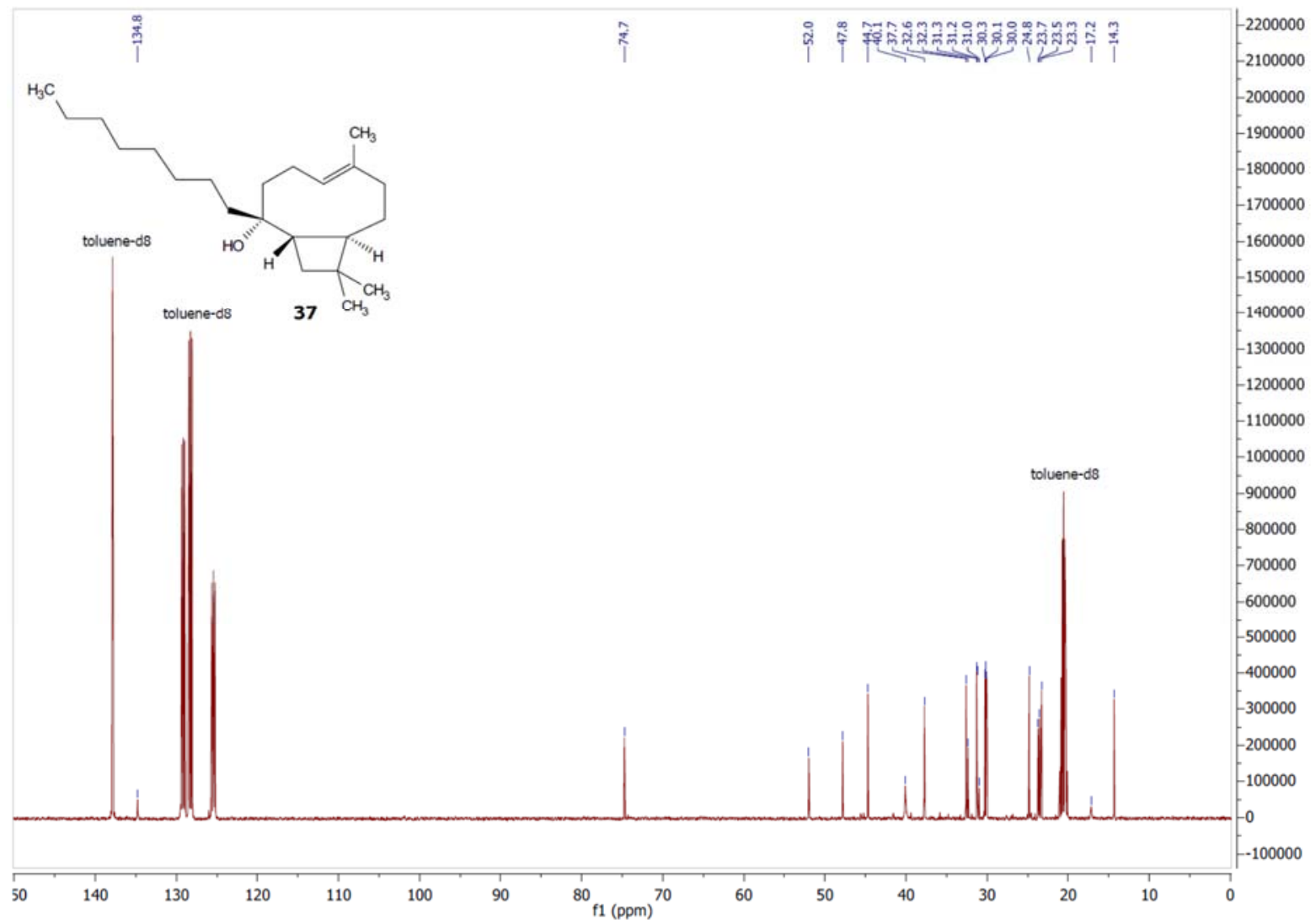




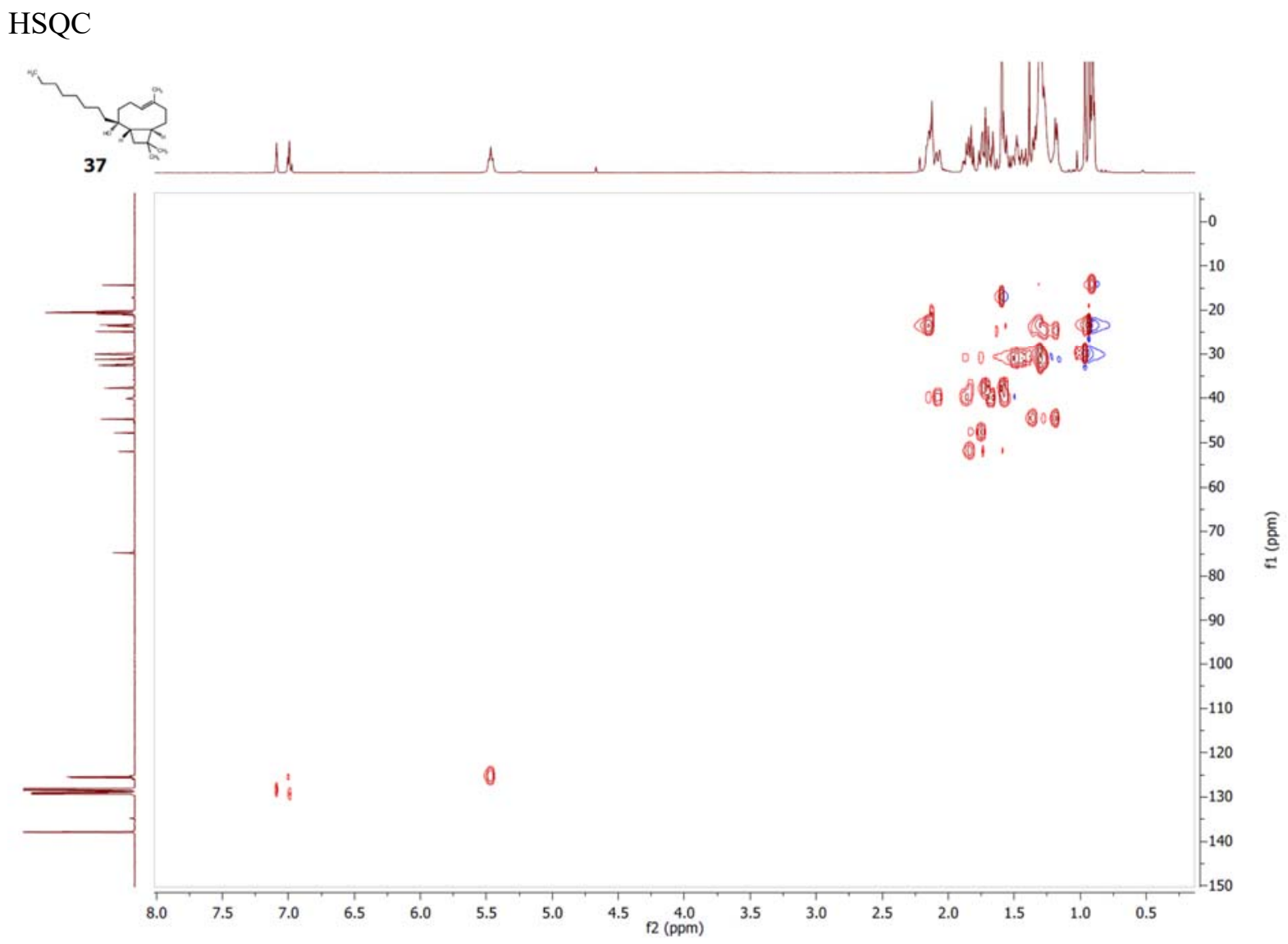

${ }^{1} \mathrm{H}$ NMR (500 MHz, toluene-d8)

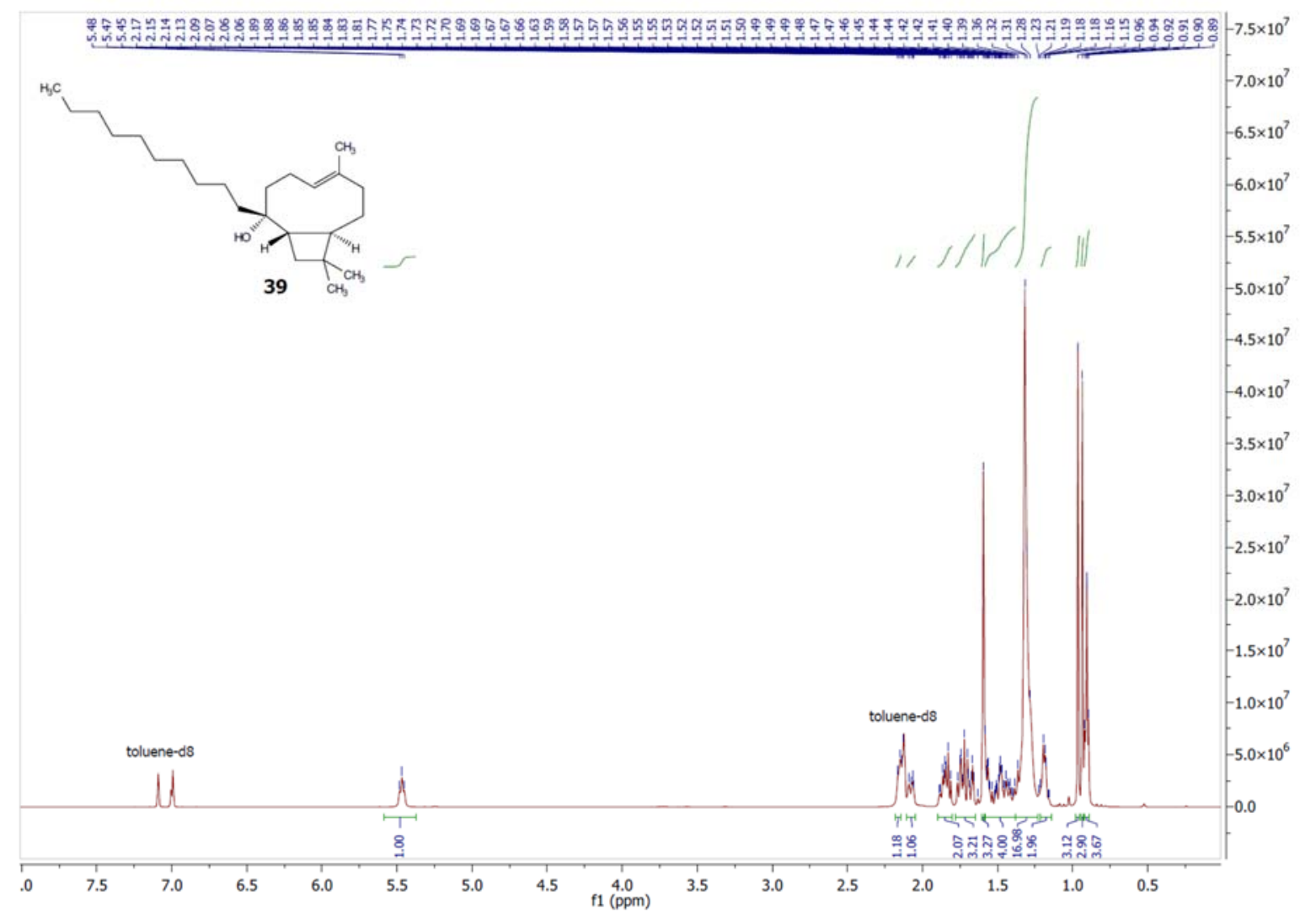


${ }^{13} \mathrm{C}$ NMR (126 MHz, toluene-d8)

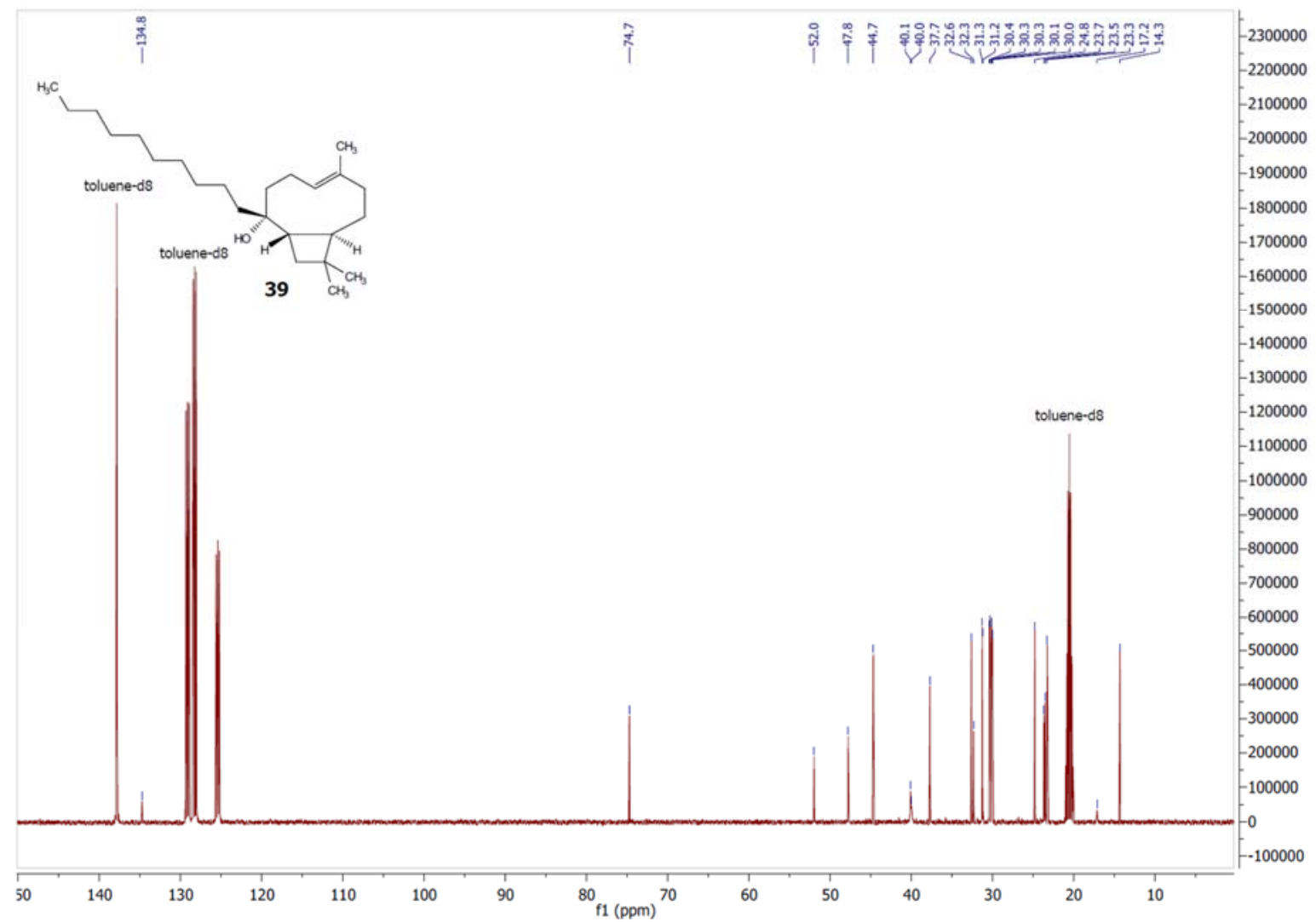

HSQC

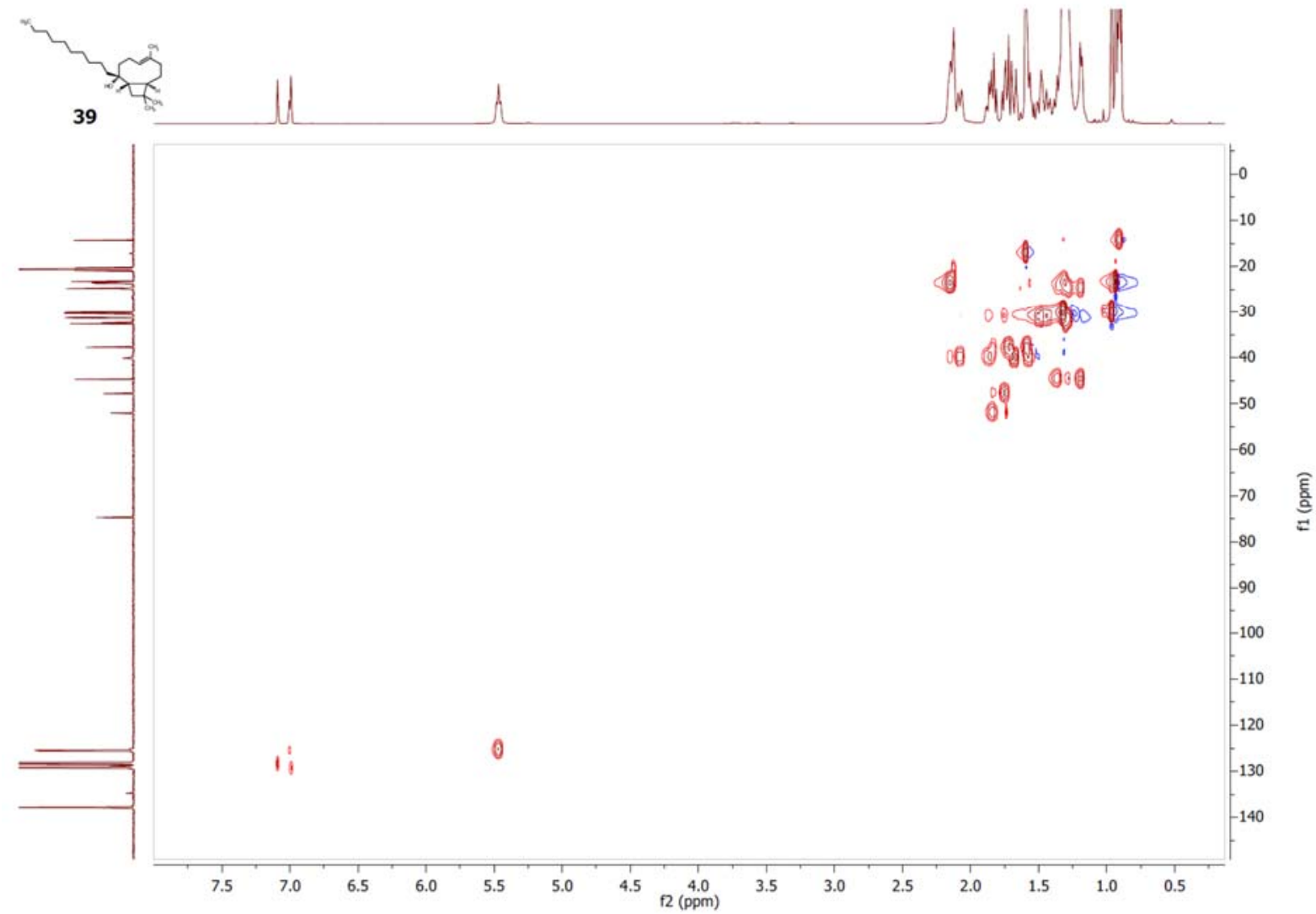




\section{Appendix D: NMR spectra of products of cyclization reaction}

${ }^{1} \mathrm{H}$ NMR (500 MHz, $\mathrm{CDCl}_{3}$ )

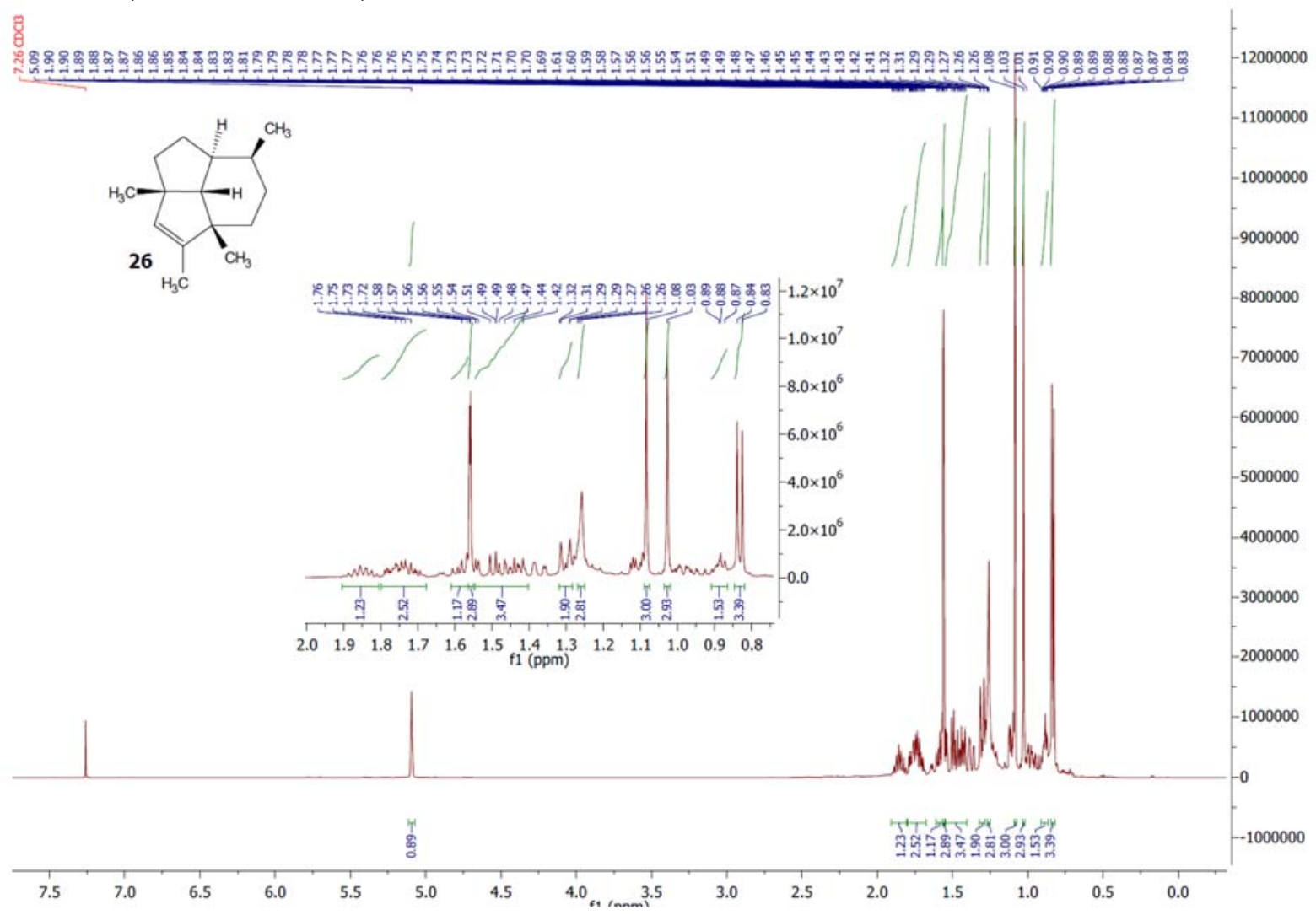

${ }^{13} \mathrm{C}$ NMR (126 MHz, $\left.\mathrm{CDCl}_{3}\right)$

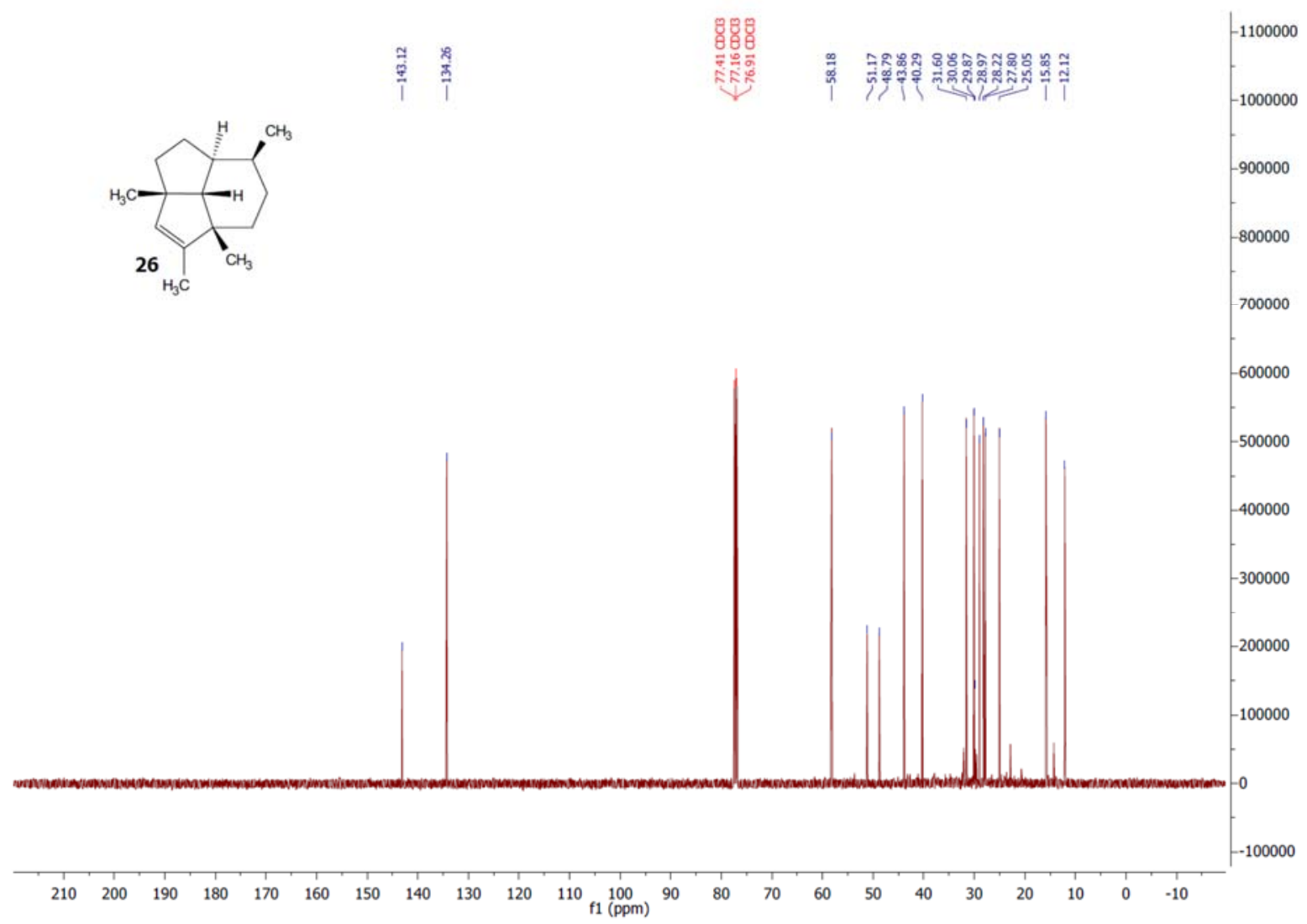


${ }^{1} \mathrm{H}$ NMR $\left(500 \mathrm{MHz}, \mathrm{CDCl}_{3}\right)$

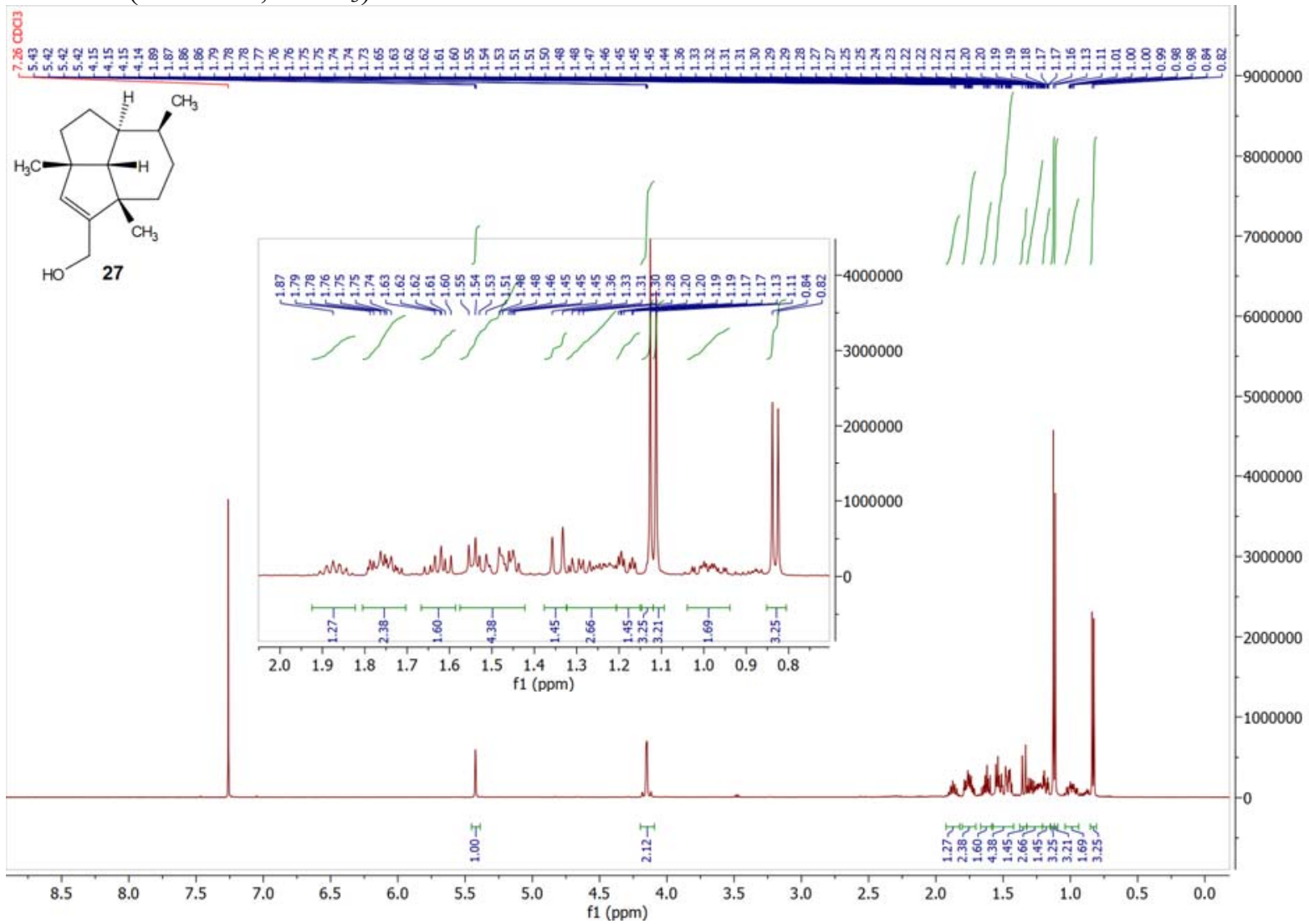

${ }^{13} \mathrm{C}$ NMR $\left(126 \mathrm{MHz}, \mathrm{CDCl}_{3}\right)$

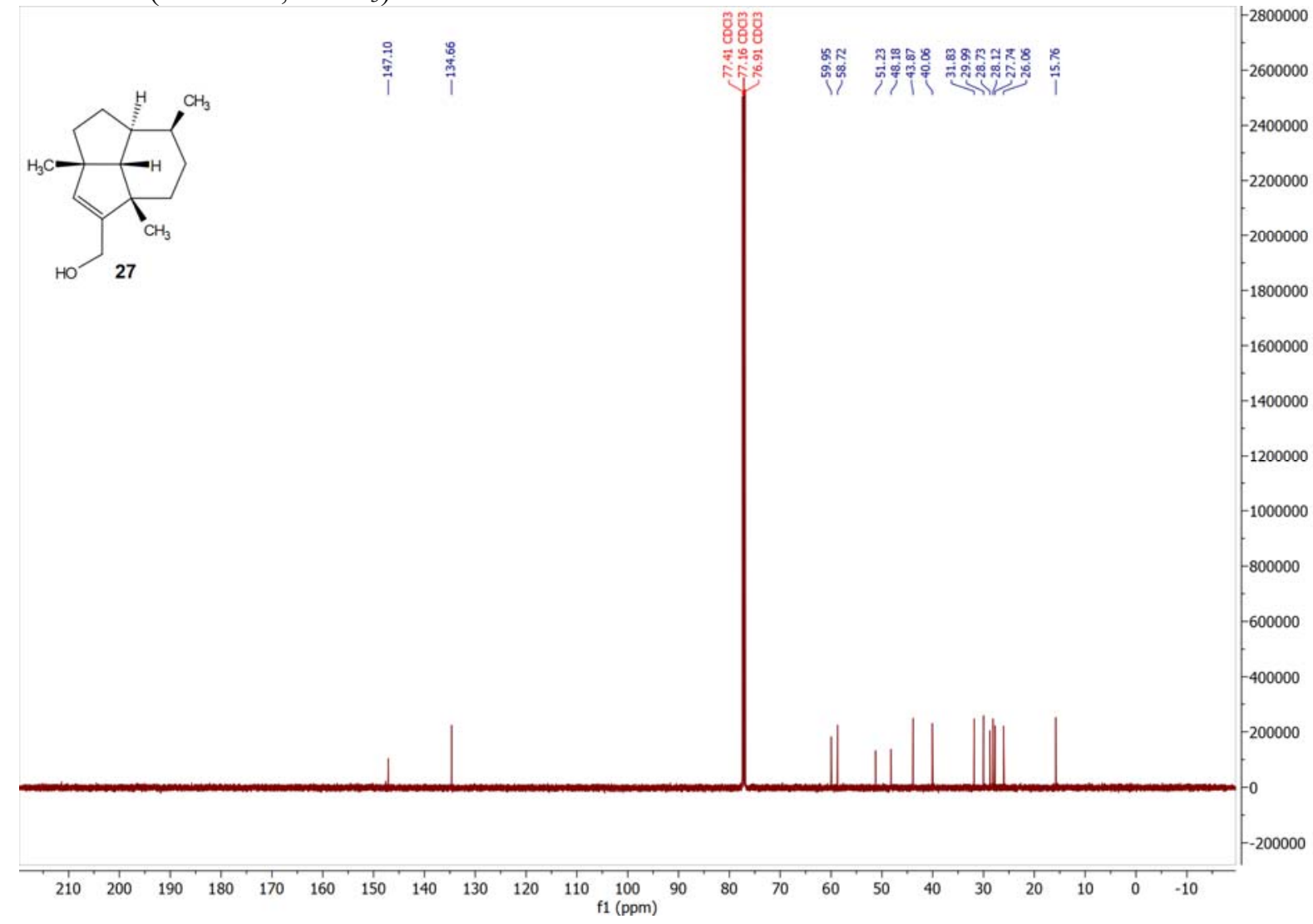


${ }^{1} \mathrm{H}$ NMR (500 MHz, $\left.\mathrm{CDCl}_{3}\right)$
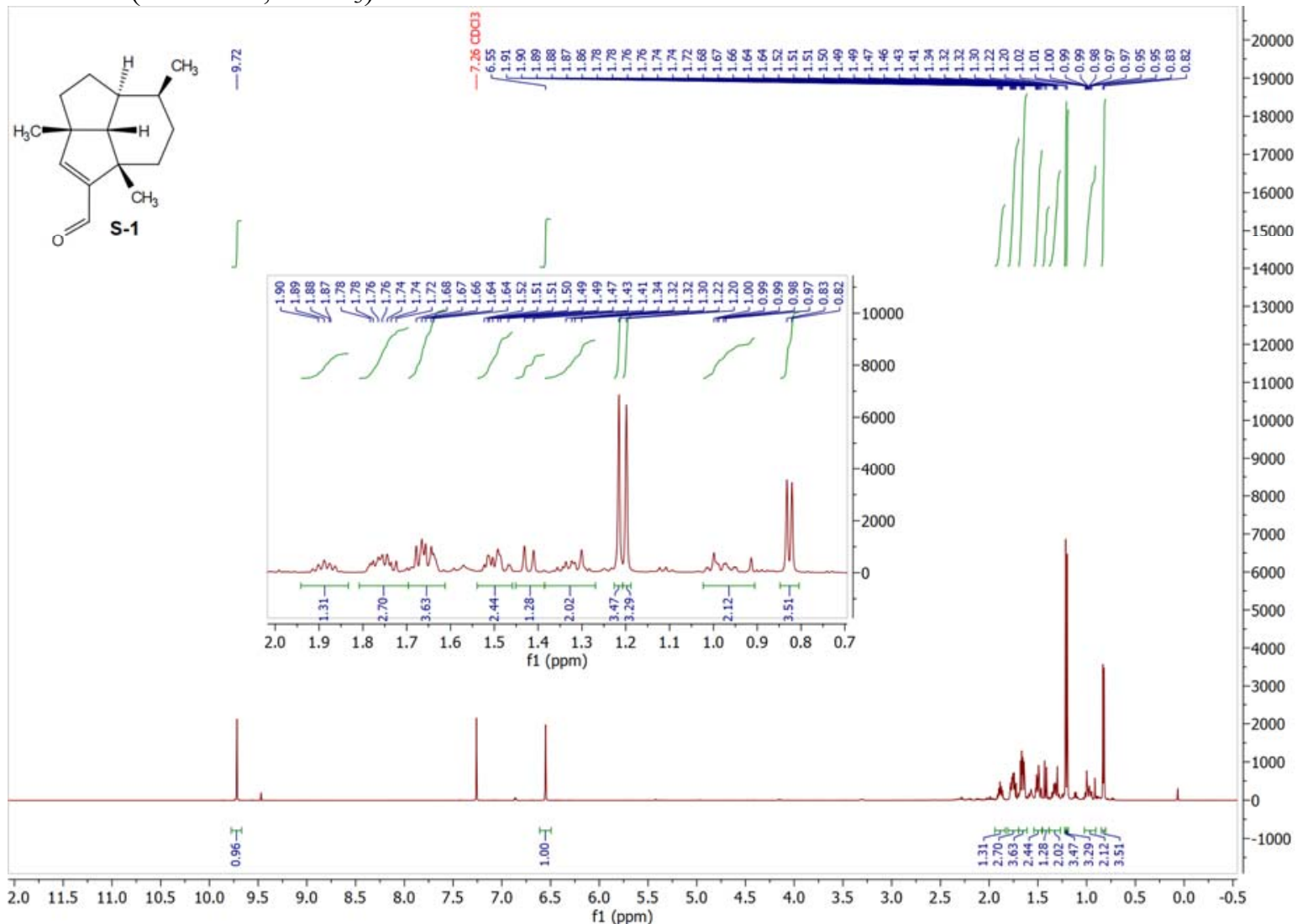

${ }^{13} \mathrm{C}$ NMR $\left(126 \mathrm{MHz}, \mathrm{CDCl}_{3}\right)$

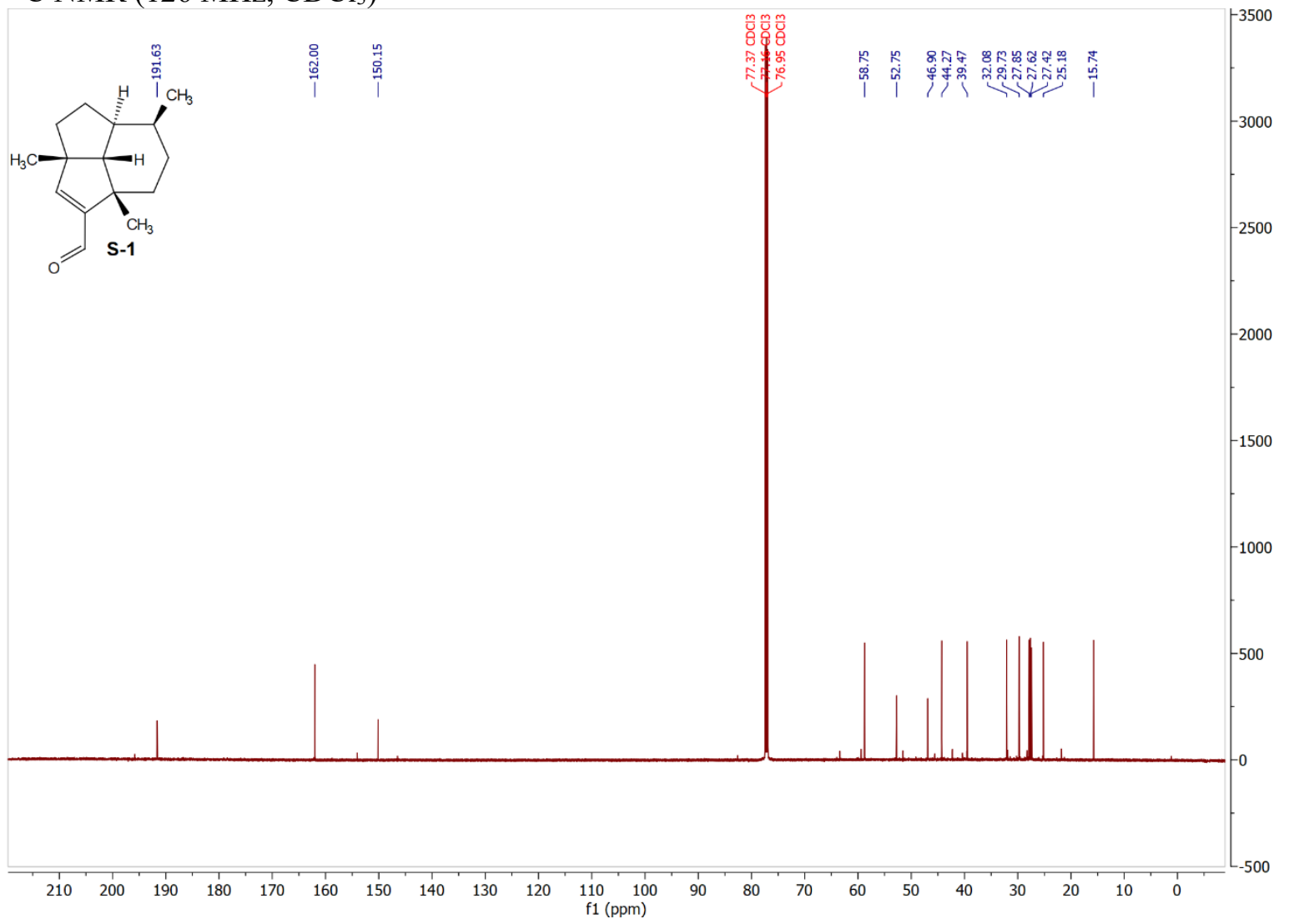


${ }^{1} \mathrm{H}$ NMR $\left(500 \mathrm{MHz}, \mathrm{CDCl}_{3}\right)$

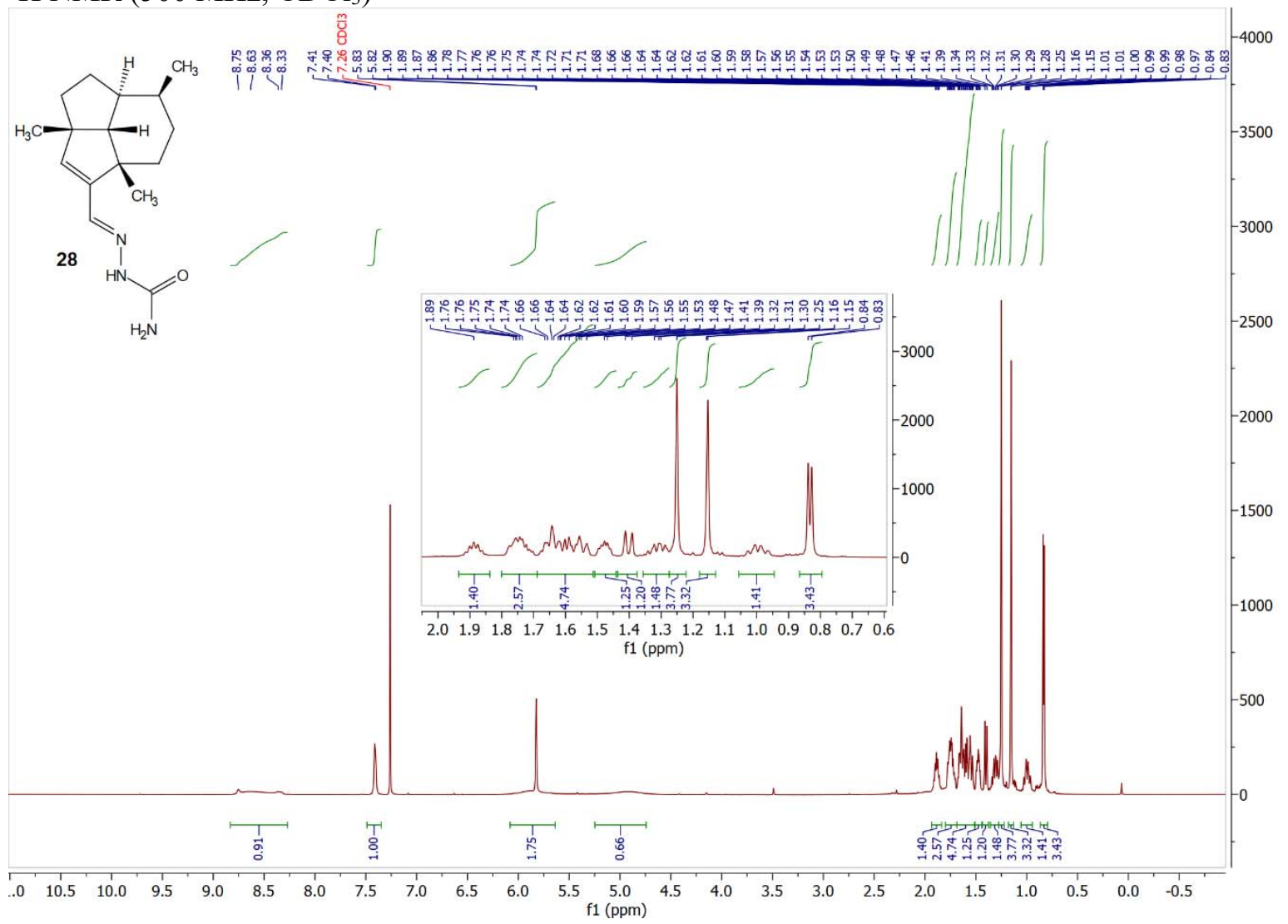

${ }^{13} \mathrm{C}$ NMR $\left(126 \mathrm{MHz}, \mathrm{CDCl}_{3}\right)$

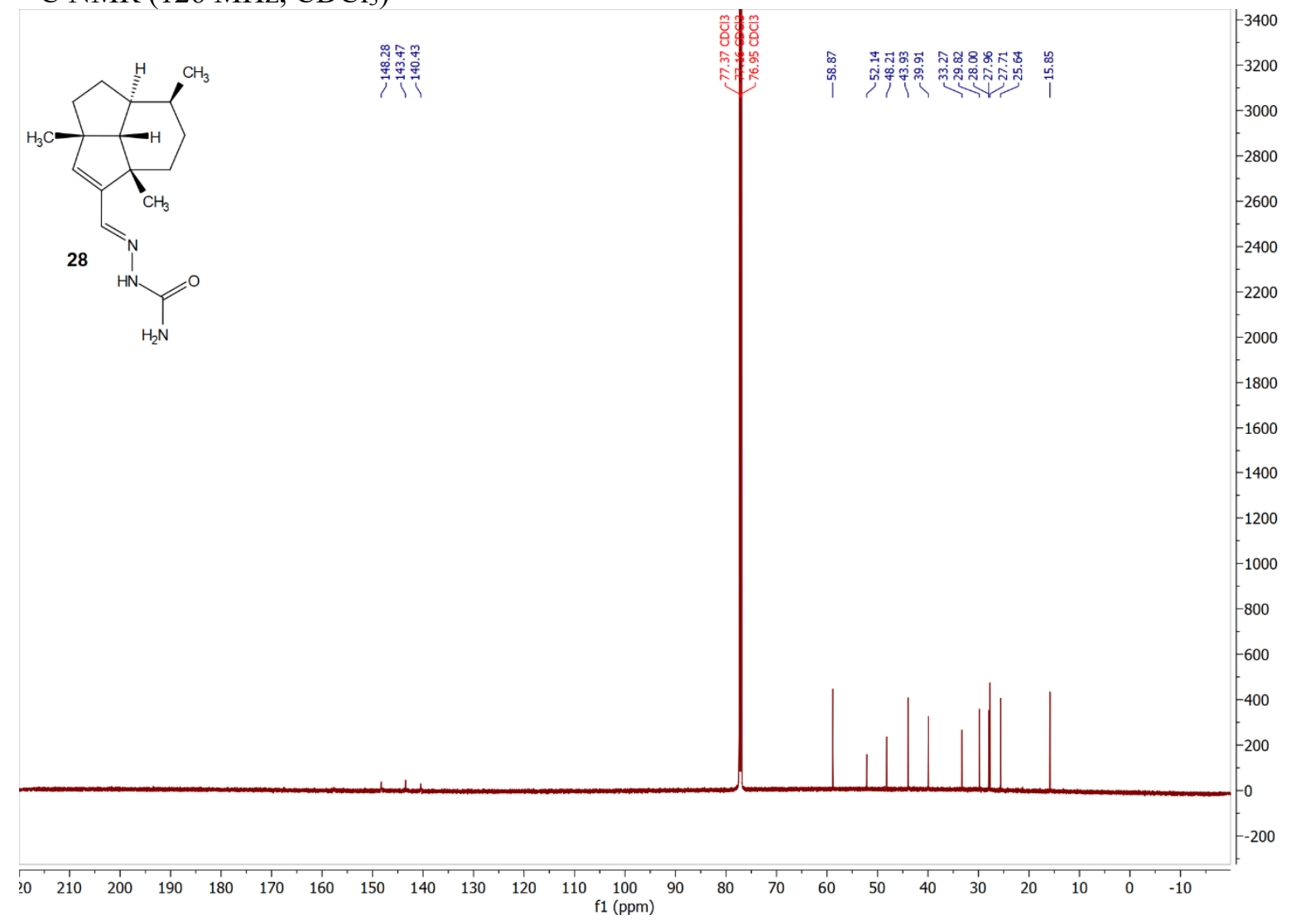


${ }^{1} \mathrm{H}$ NMR (500 MHz, $\left.\mathrm{CDCl}_{3}\right)$

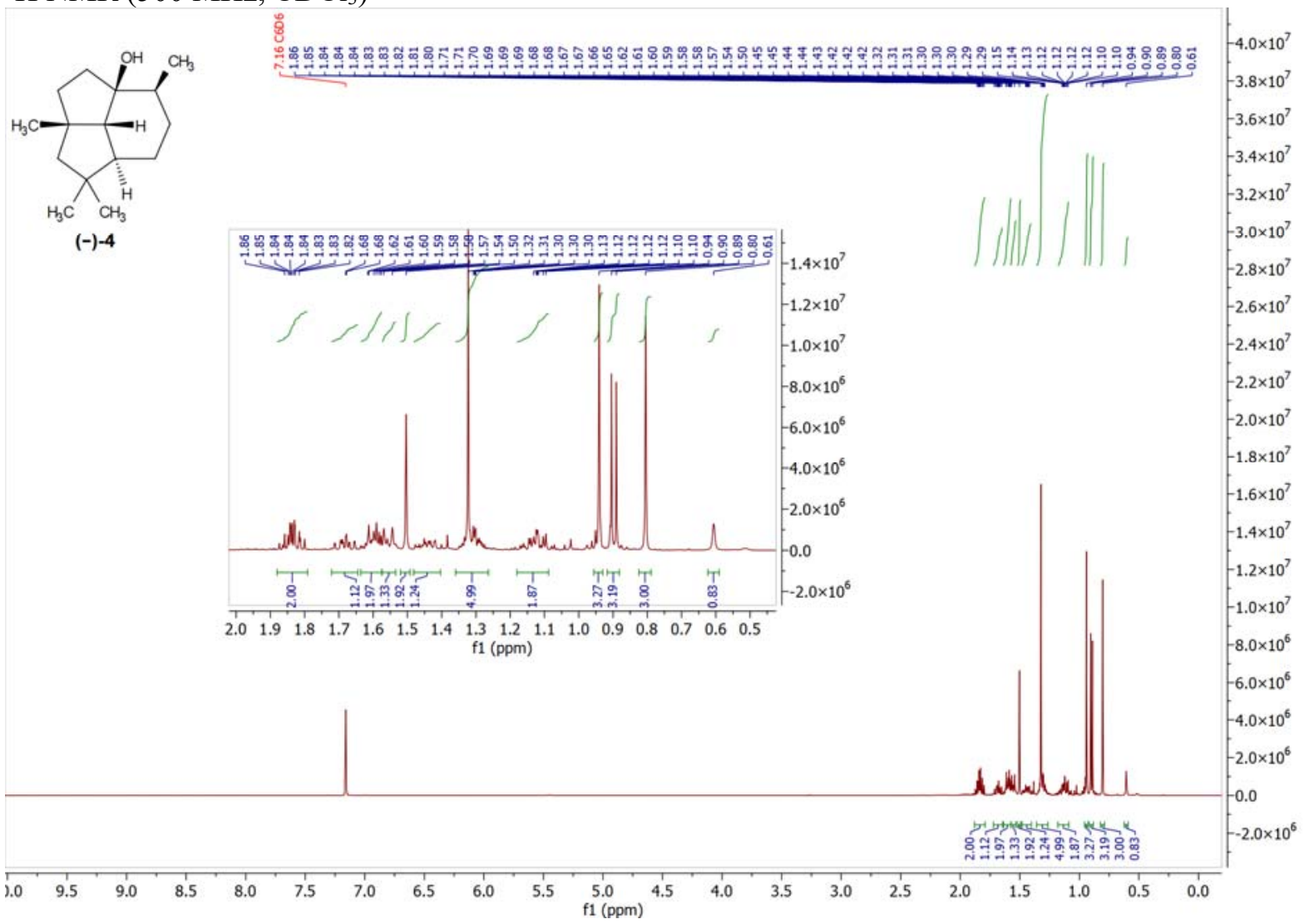

${ }^{13} \mathrm{C}$ NMR (126 MHz, $\left.\mathrm{CDCl}_{3}\right)$

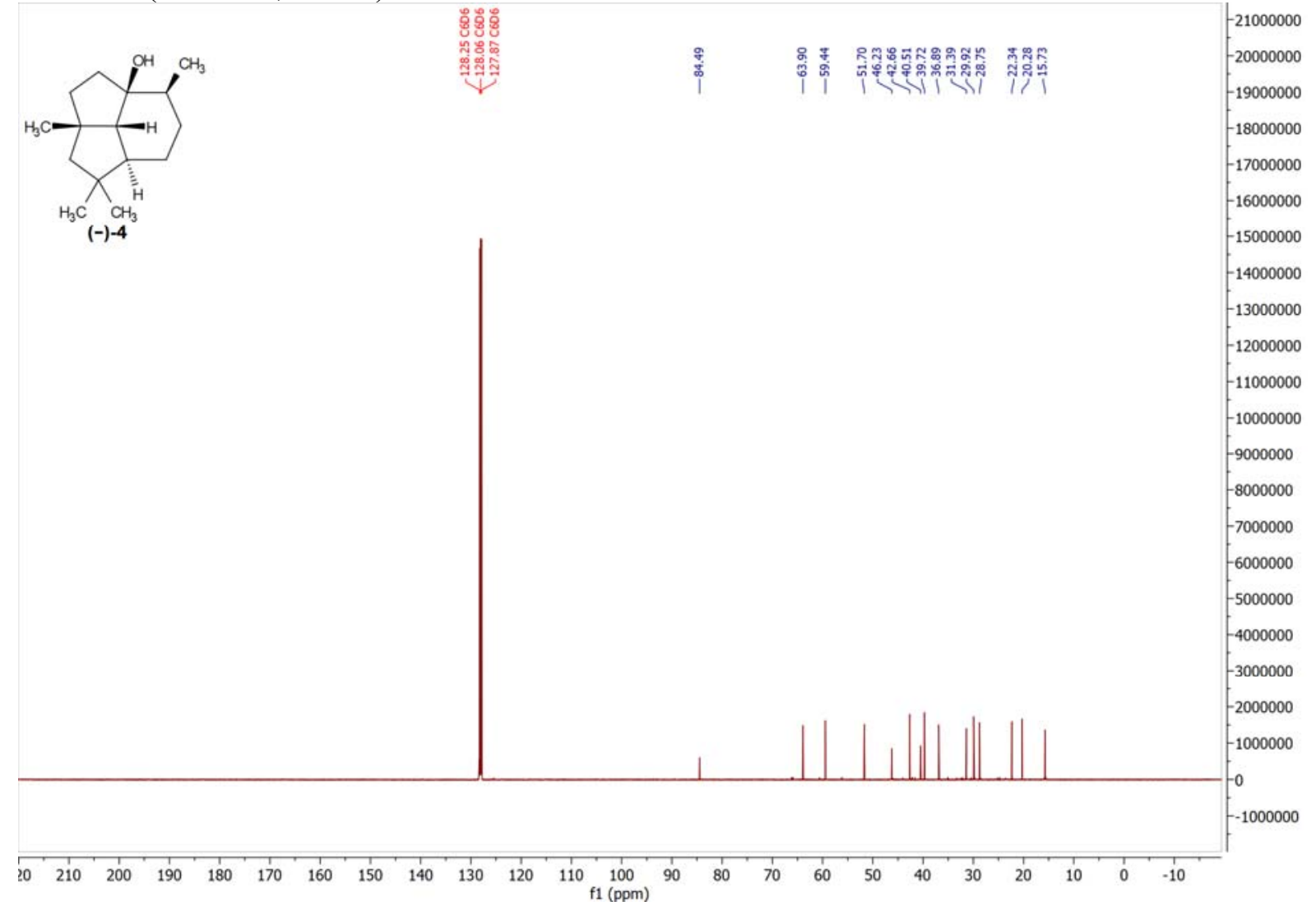


${ }^{1} \mathrm{H}$ NMR (500 MHz, $\left.\mathrm{CDCl}_{3}\right)$

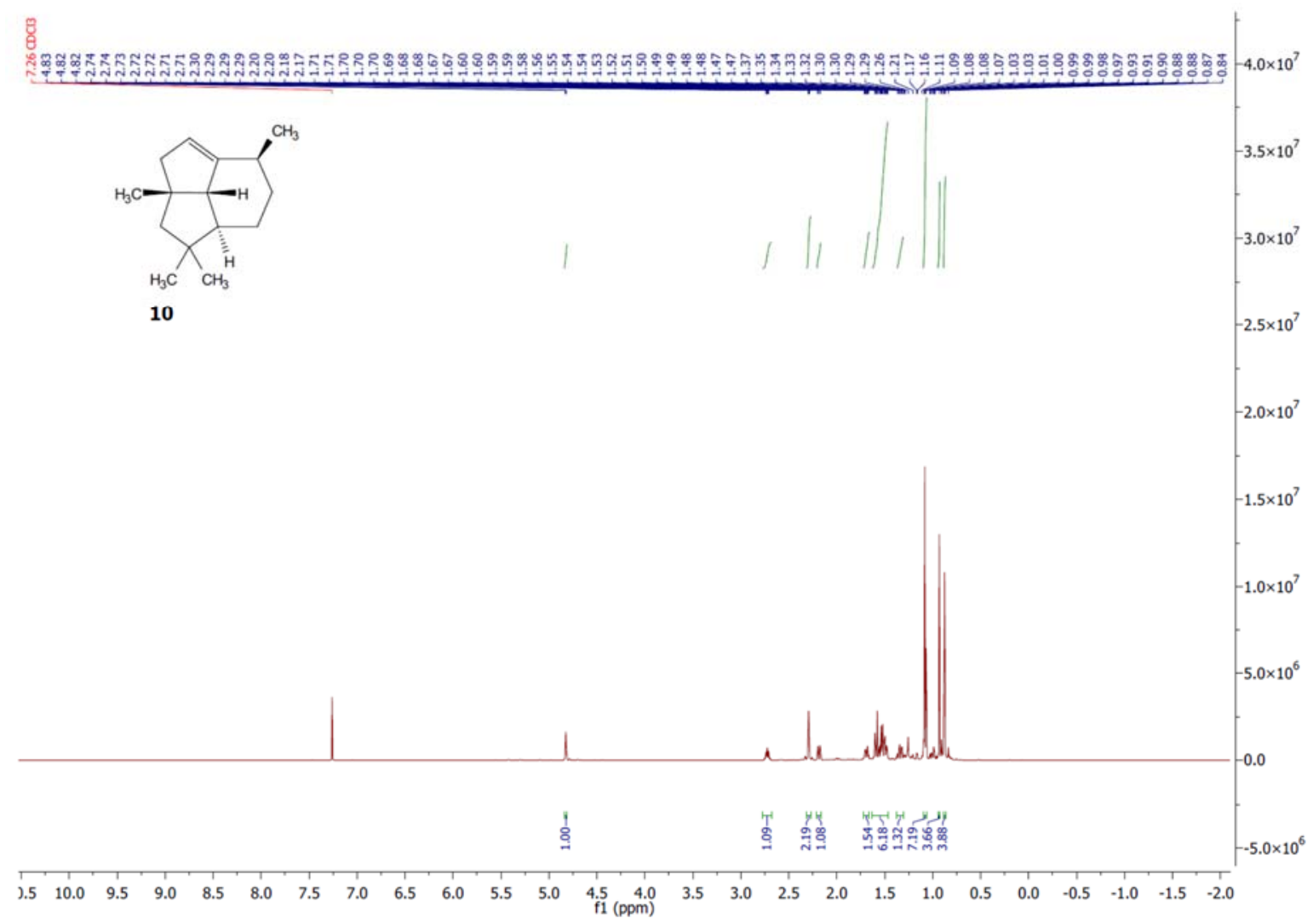

${ }^{13} \mathrm{C}$ NMR (126 MHz, $\left.\mathrm{CDCl}_{3}\right)$

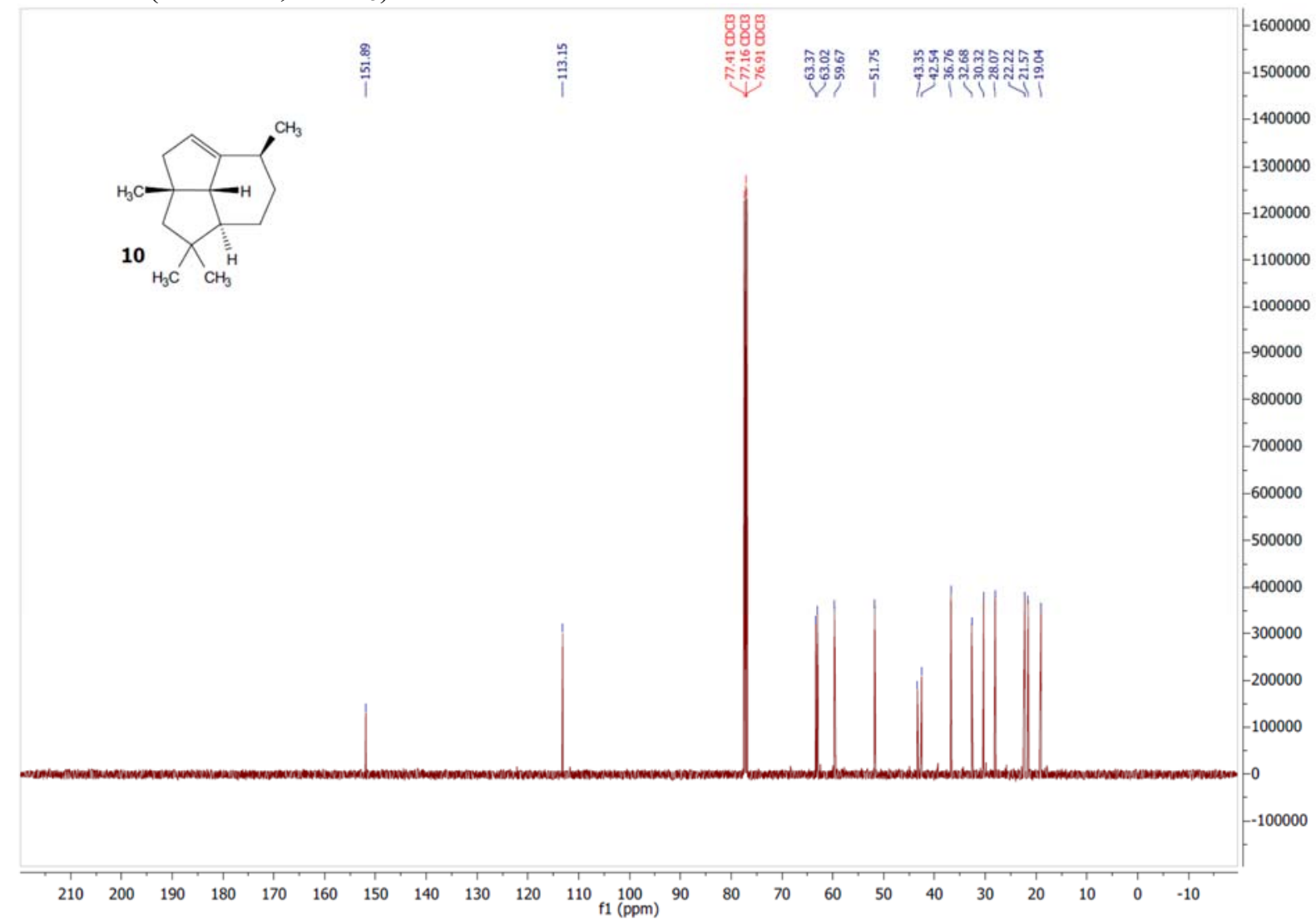


${ }^{1} \mathrm{H}$ NMR (500 MHz, $\left.\mathrm{CDCl}_{3}\right)$

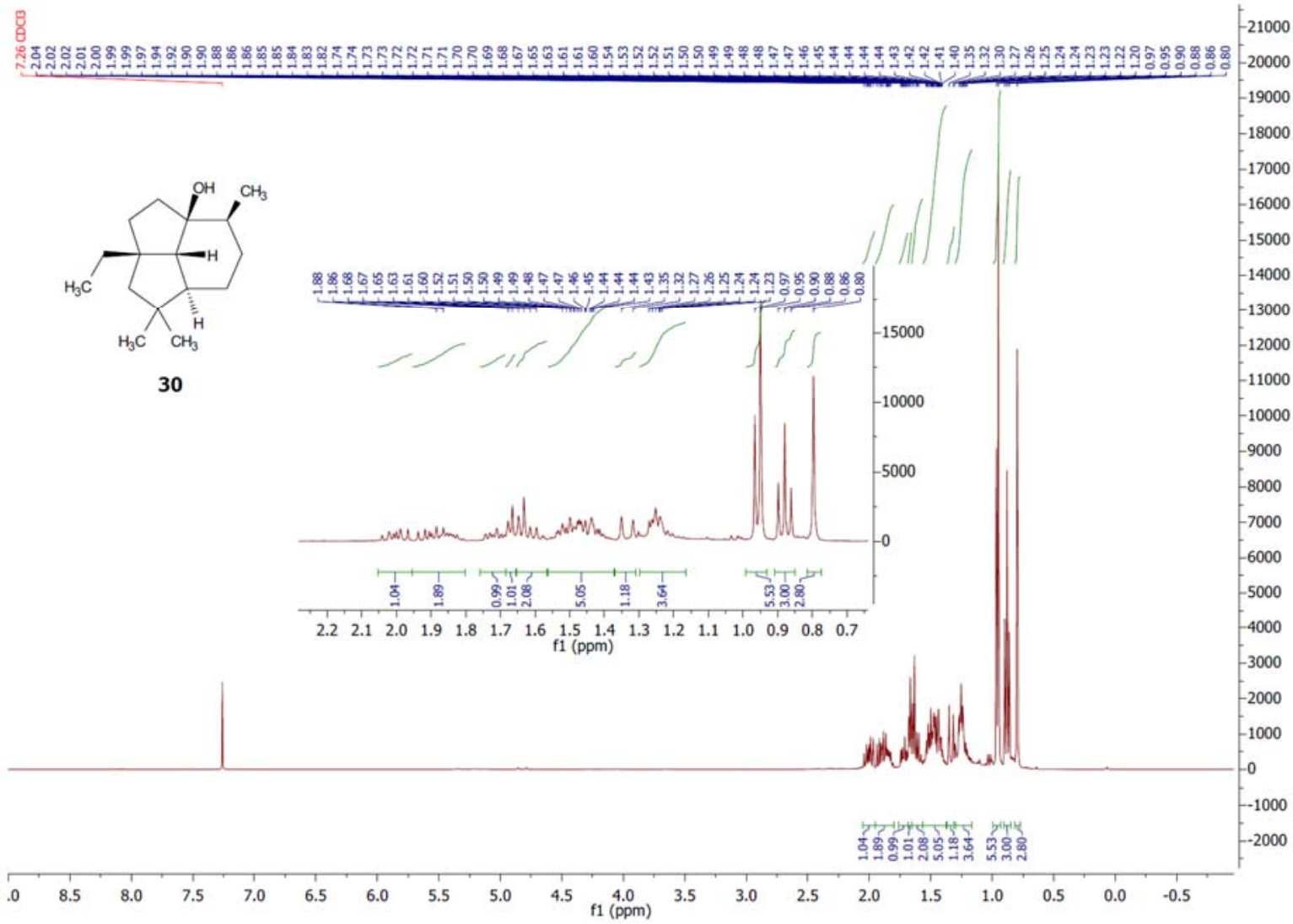

${ }^{13} \mathrm{C}$ NMR (126 MHz, $\left.\mathrm{CDCl}_{3}\right)$

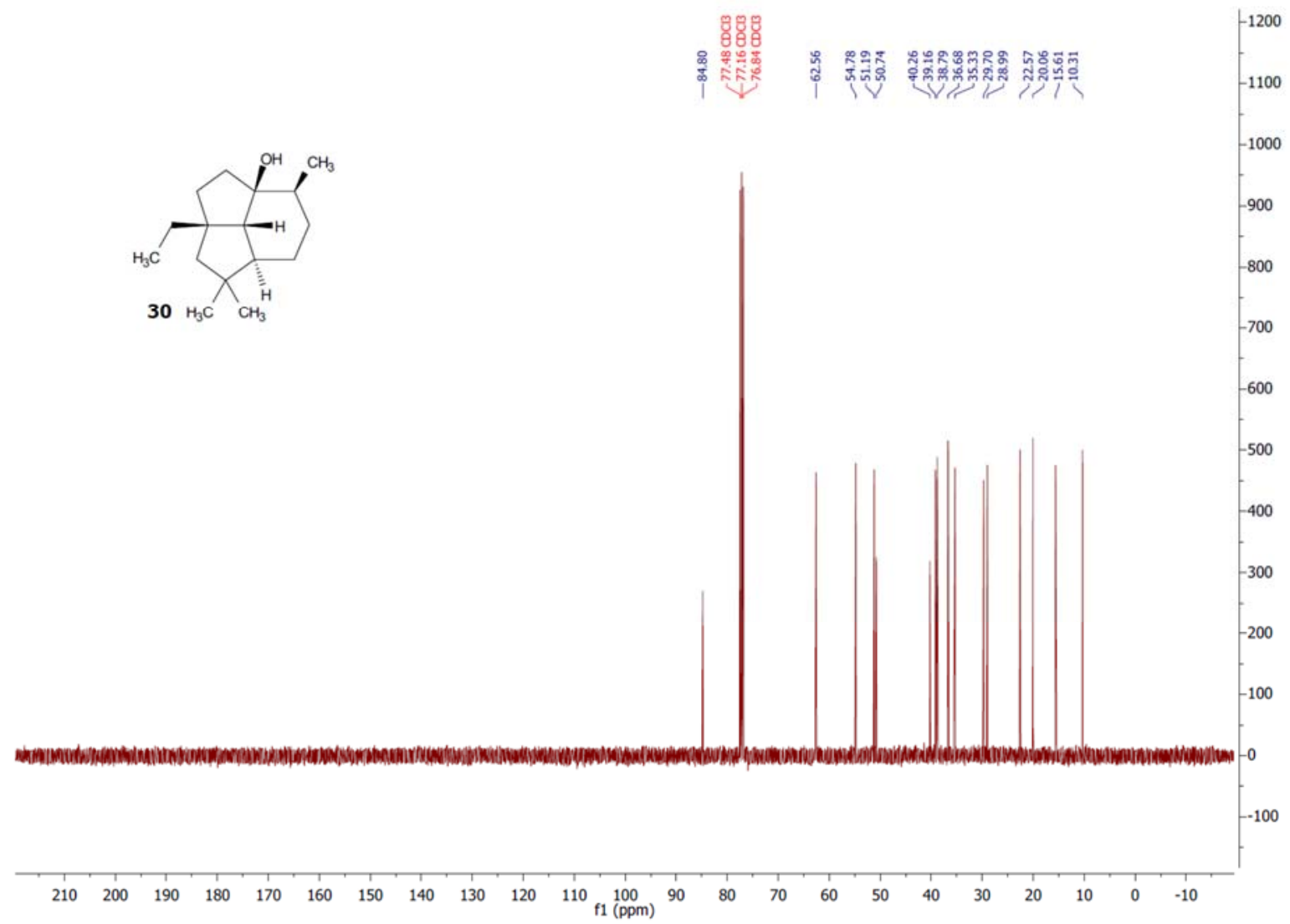


${ }^{1} \mathrm{H}$ NMR (500 MHz, $\left.\mathrm{CDCl}_{3}\right)$

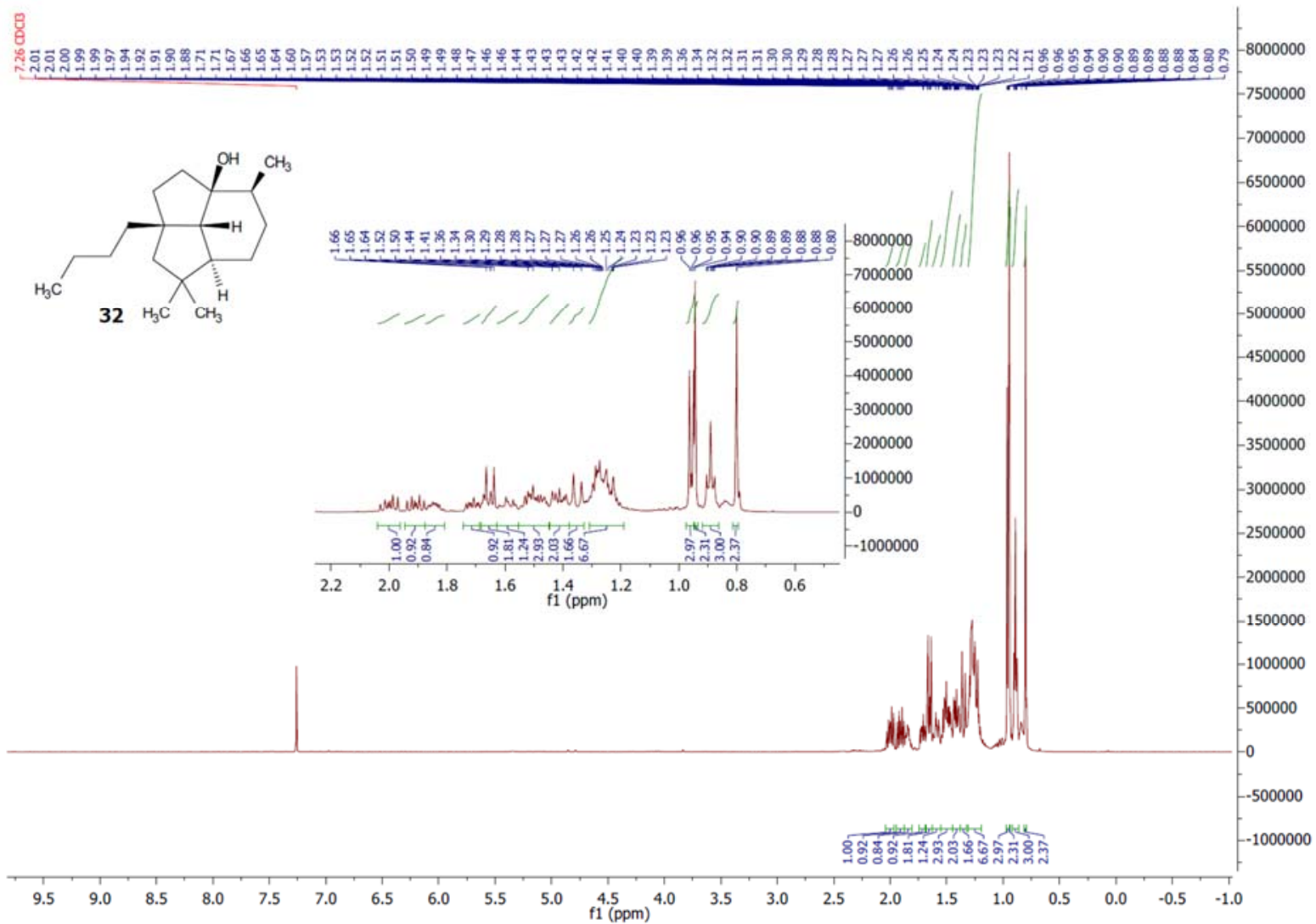

${ }^{13} \mathrm{C}$ NMR (126 MHz, $\mathrm{CDCl}_{3}$ )

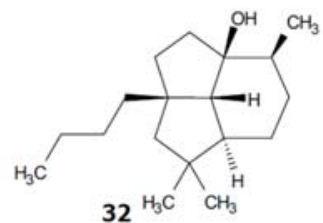

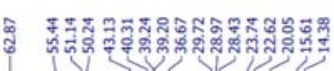

$-600000$

50000

500000

450000

400000

$-350000$

300000

$-250000$

$-200000$

$-150000$

$-100000$

$-50000$

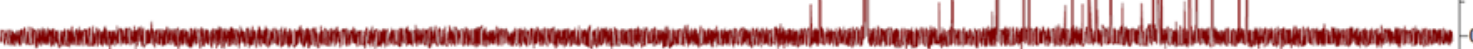

$-0$

$\begin{array}{llllllllllllllllllllllll}210 & 200 & 190 & 180 & 170 & 160 & 150 & 140 & 130 & 120 & 110 & 100 & 90 & 80 & 70 & 60 & 50 & 40 & 30 & 20 & 10 & 0 & -10\end{array}$ 
${ }^{1} \mathrm{H}$ NMR $\left(500 \mathrm{MHz}, \mathrm{CDCl}_{3}\right)$

量

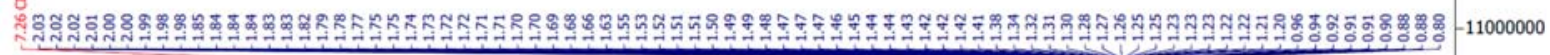

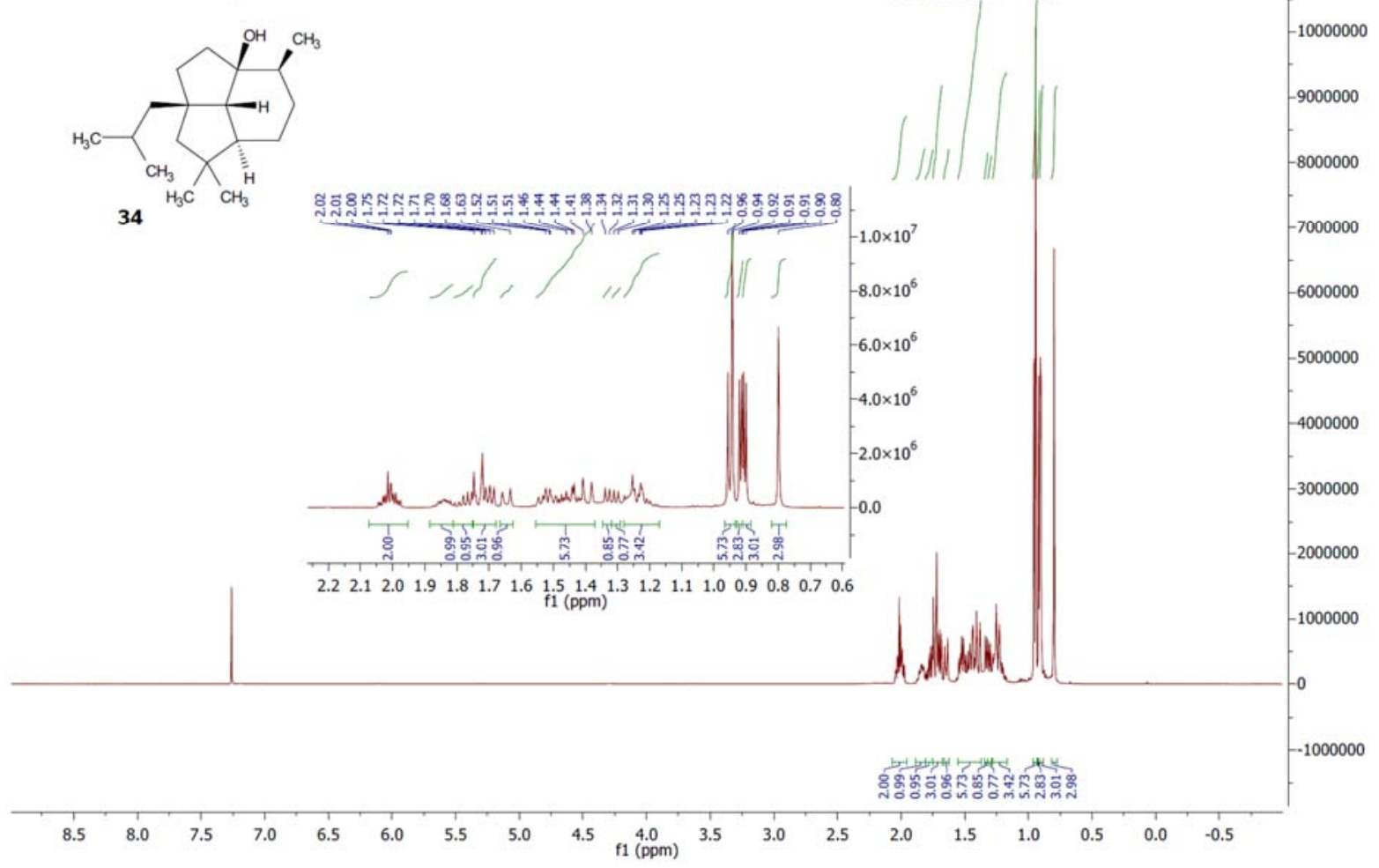

${ }^{13} \mathrm{C}$ NMR $\left(126 \mathrm{MHz}, \mathrm{CDCl}_{3}\right)$

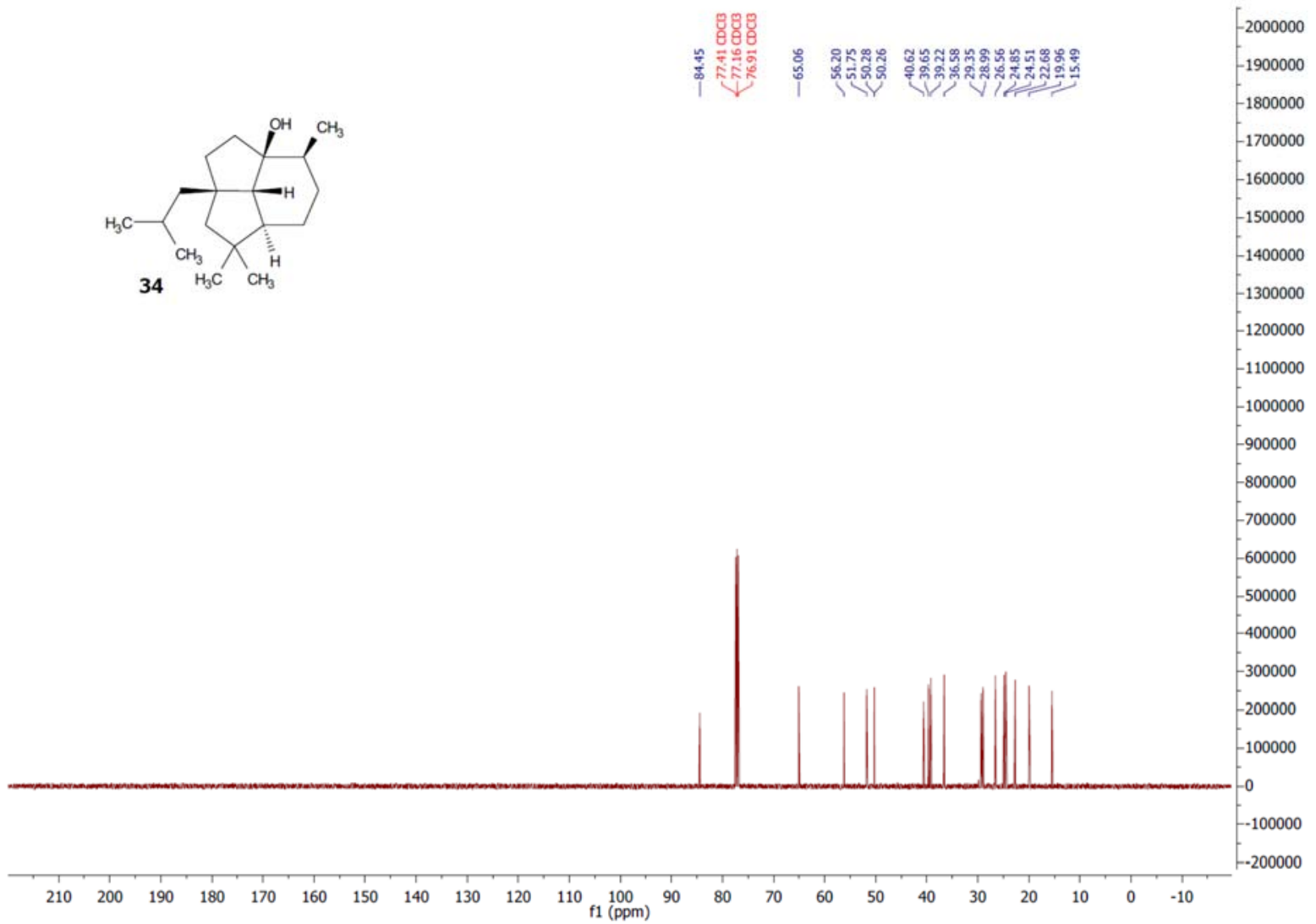




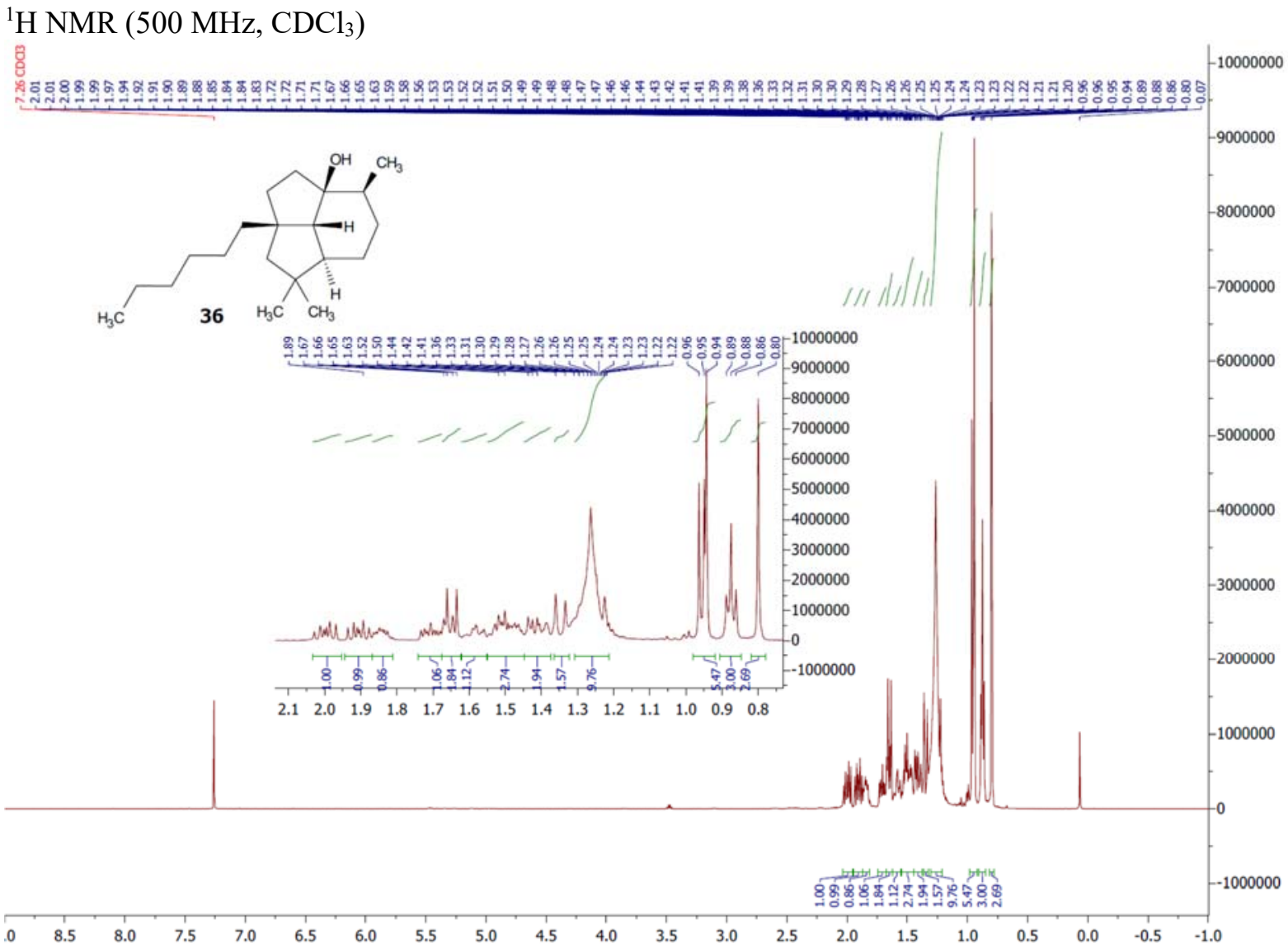

${ }^{13} \mathrm{C}$ NMR $\left(126 \mathrm{MHz}, \mathrm{CDCl}_{3}\right)$
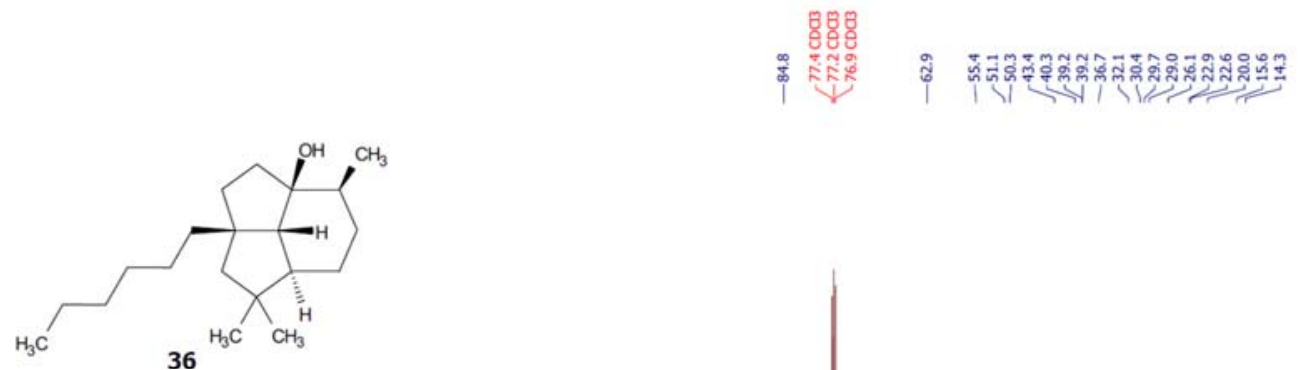

1.

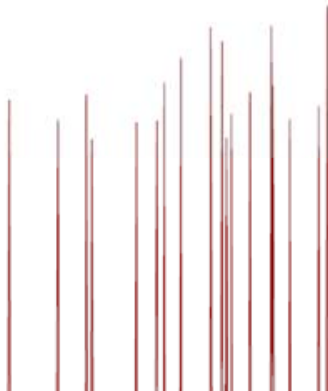


${ }^{1} \mathrm{H}$ NMR (500 MHz, $\left.\mathrm{CDCl}_{3}\right)$

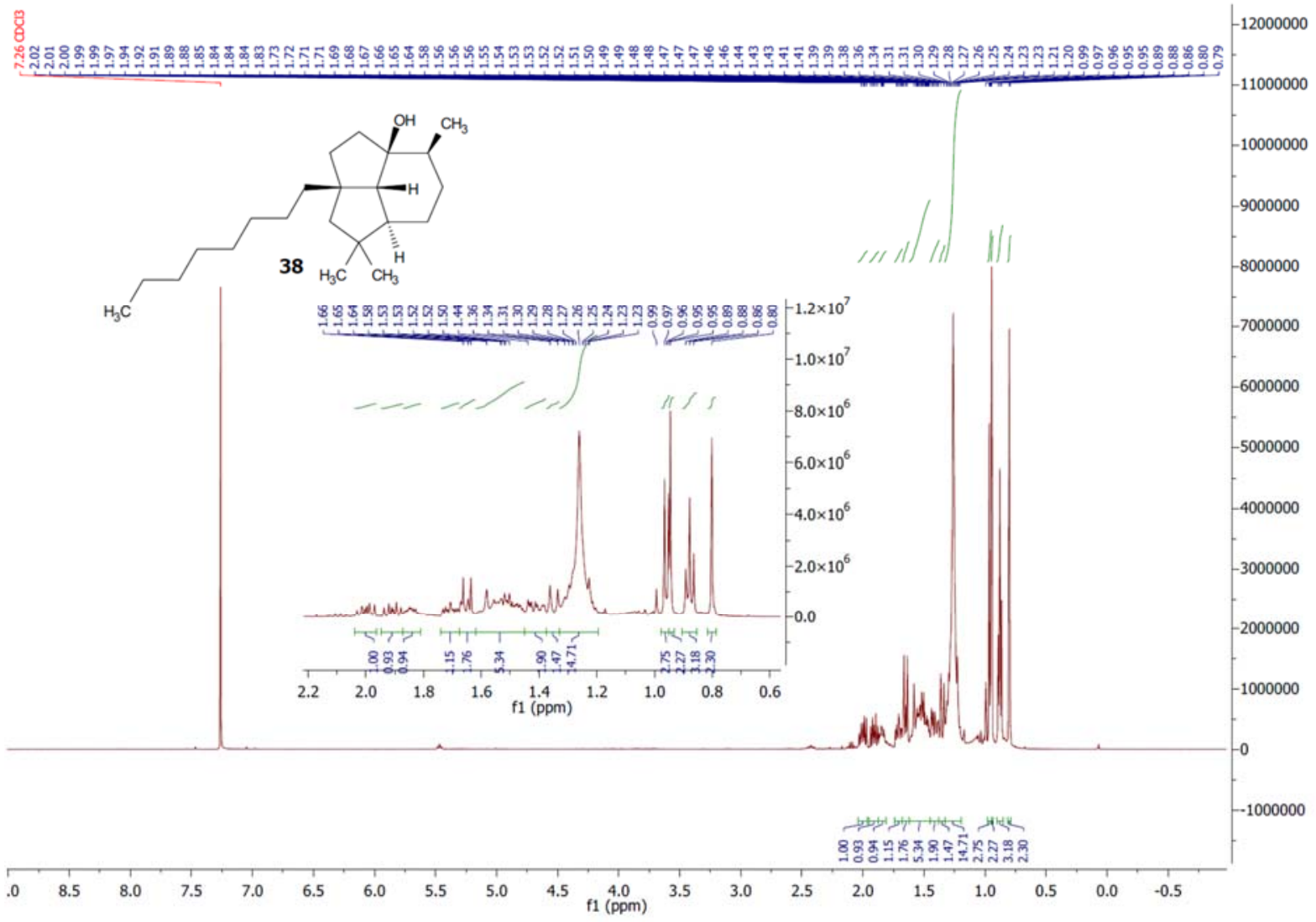

${ }^{13} \mathrm{C}$ NMR (126 MHz, $\left.\mathrm{CDCl}_{3}\right)$
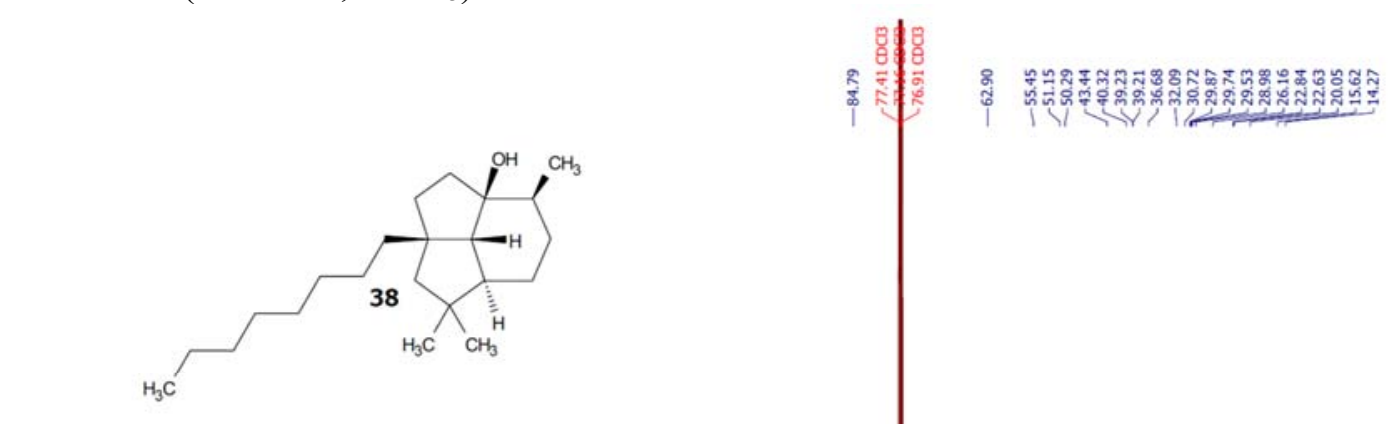

$-900000$ $-850000$ $-800000$ $-750000$ $-700000$ $-650000$ $-600000$ $-550000$ $-500000$ $-450000$ $-400000$ $-350000$ $-300000$ $-250000$ $-200000$ $-150000$ $-100000$ $-50000$

$\begin{array}{lllllllllllllllllllllll}210 & 200 & 190 & 180 & 170 & 160 & 150 & 140 & 130 & 120 & 110 & 100 & 90 & 80 & 70 & 60 & 50 & 40 & 30 & 20 & 10 & 0 & -10\end{array}$ 
Appendix E: DEPT and 2D-NMR spectra for $\left(\left(2 \mathrm{a} S, 2 \mathrm{a}^{1} S, 4 \mathrm{a} S, 7 S, 7 \mathrm{a} S\right)-2 \mathrm{a}, 4 \mathrm{a}, 7\right.$-trimethyl2,2a,2a1,4a,5,6,7,7a-octahydro-1H-cyclopenta[cd]inden-4-yl)methanol (27)

DEPT

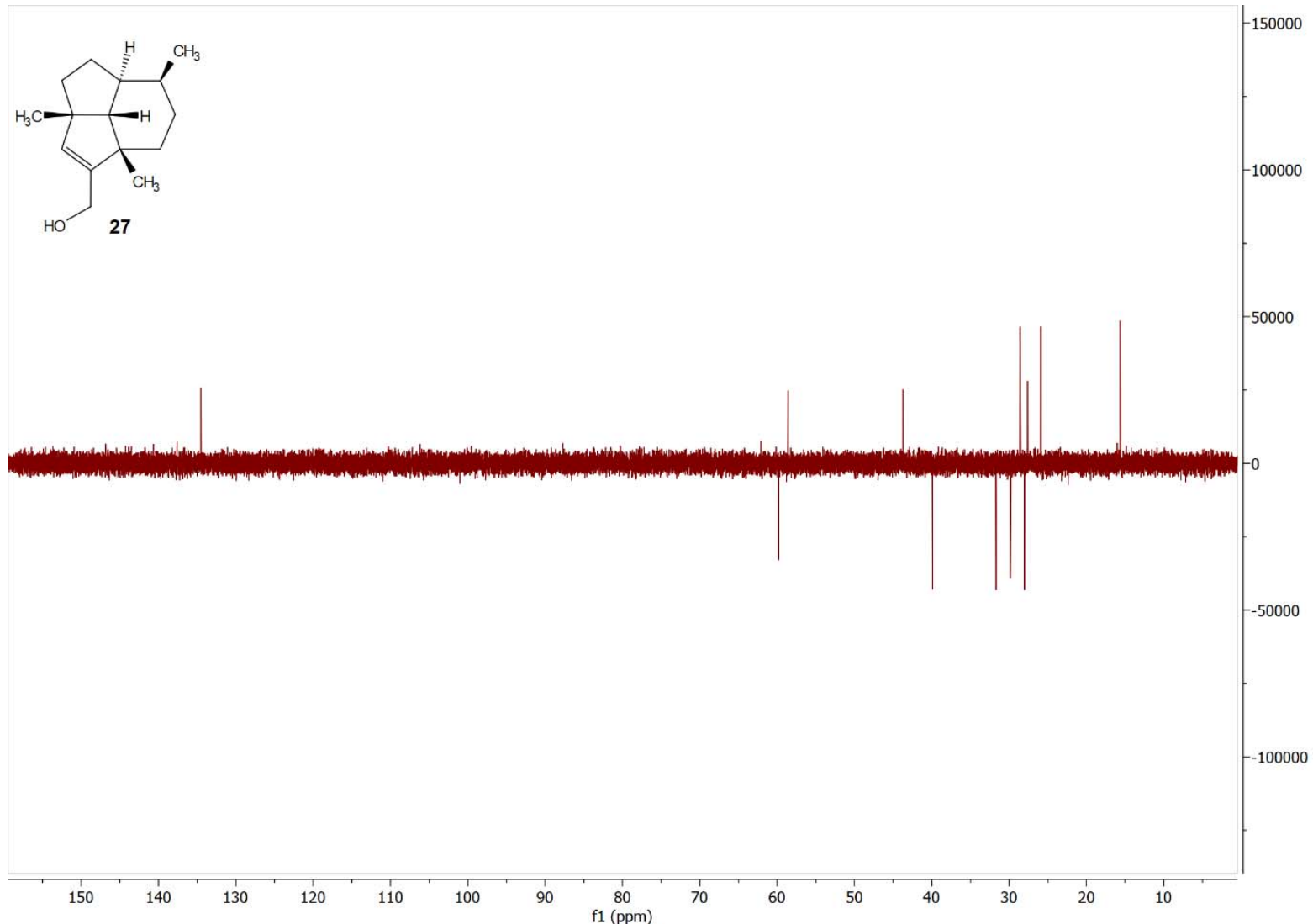

$\operatorname{COSY}$

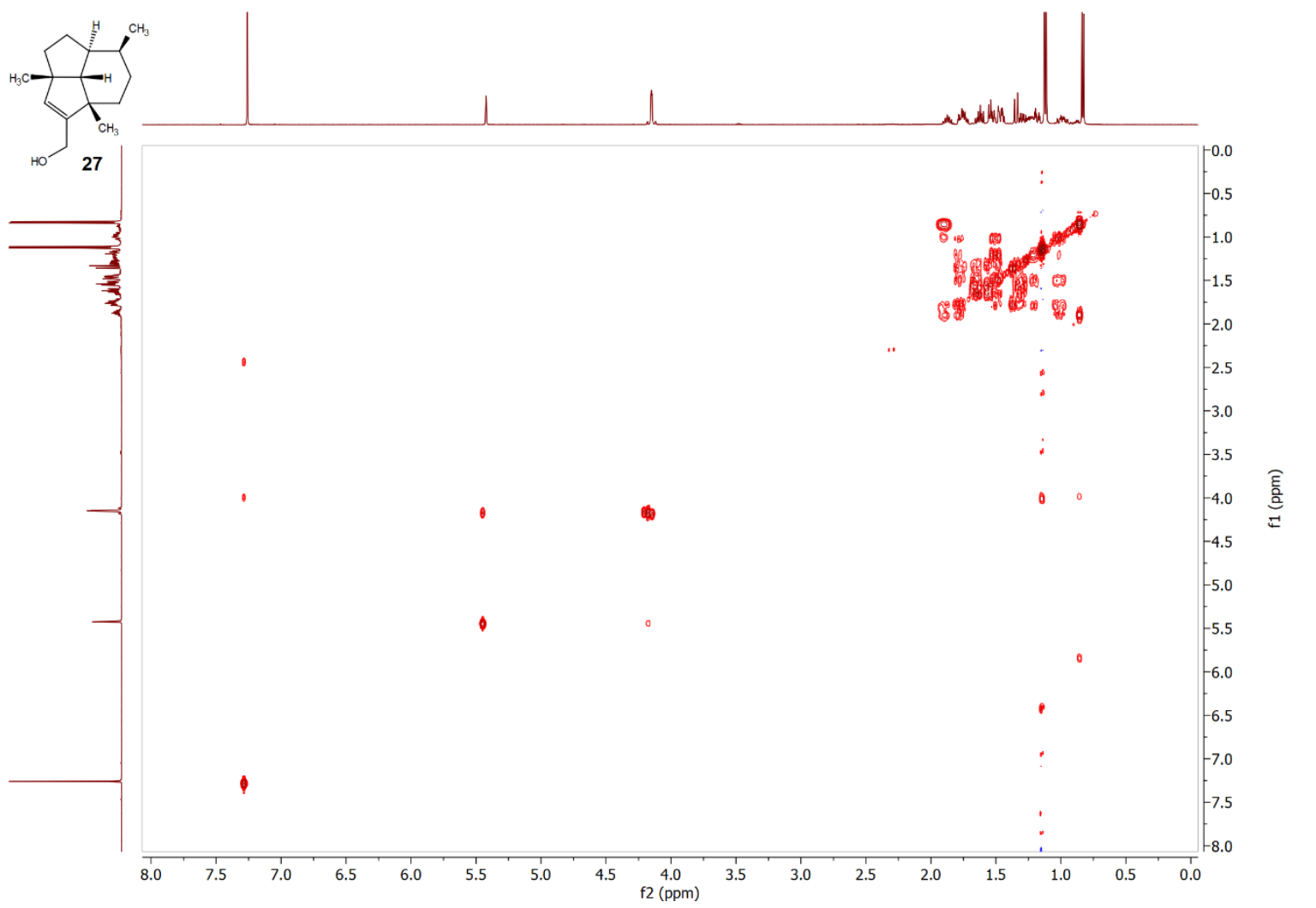


HMQC

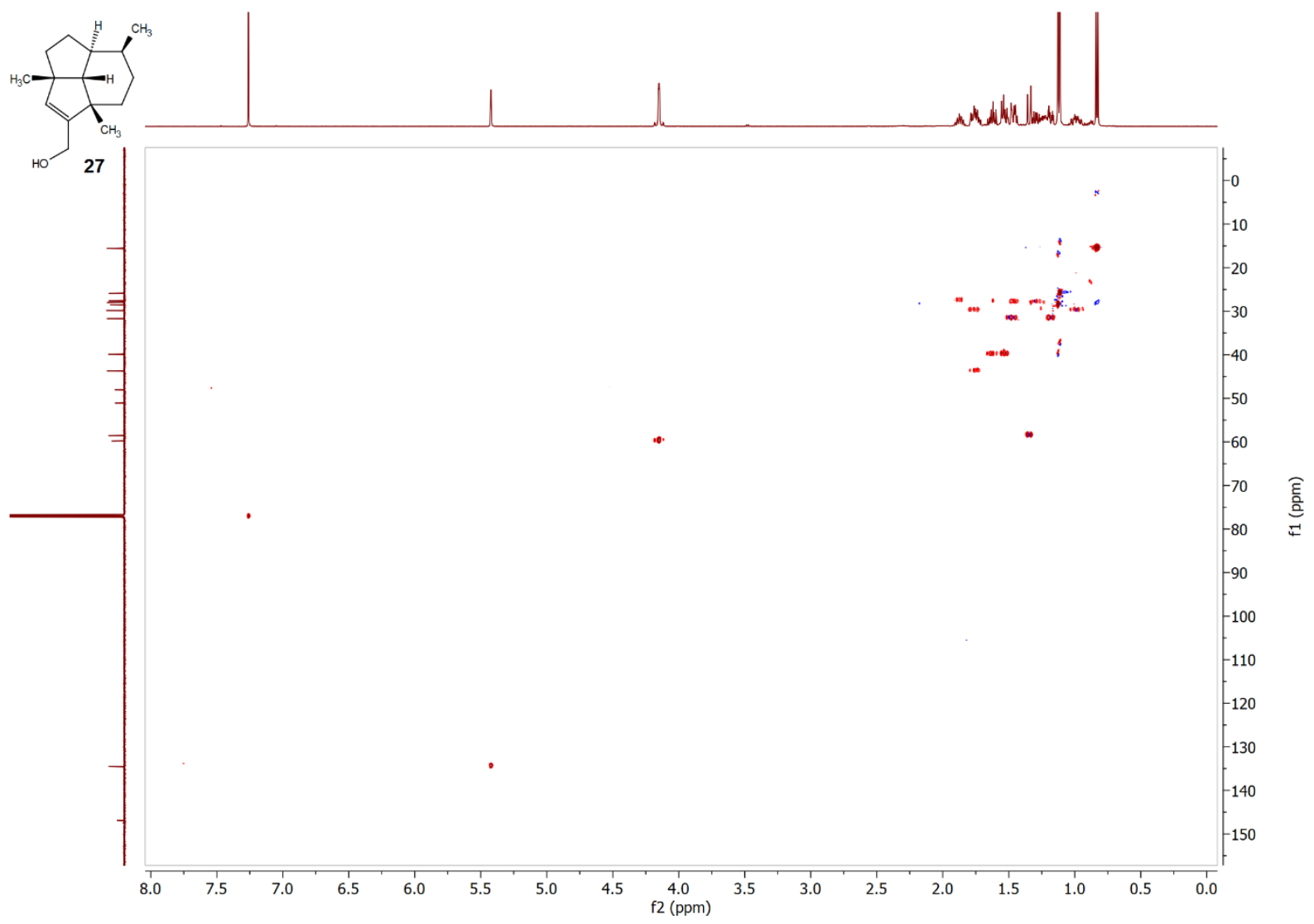

HMBC

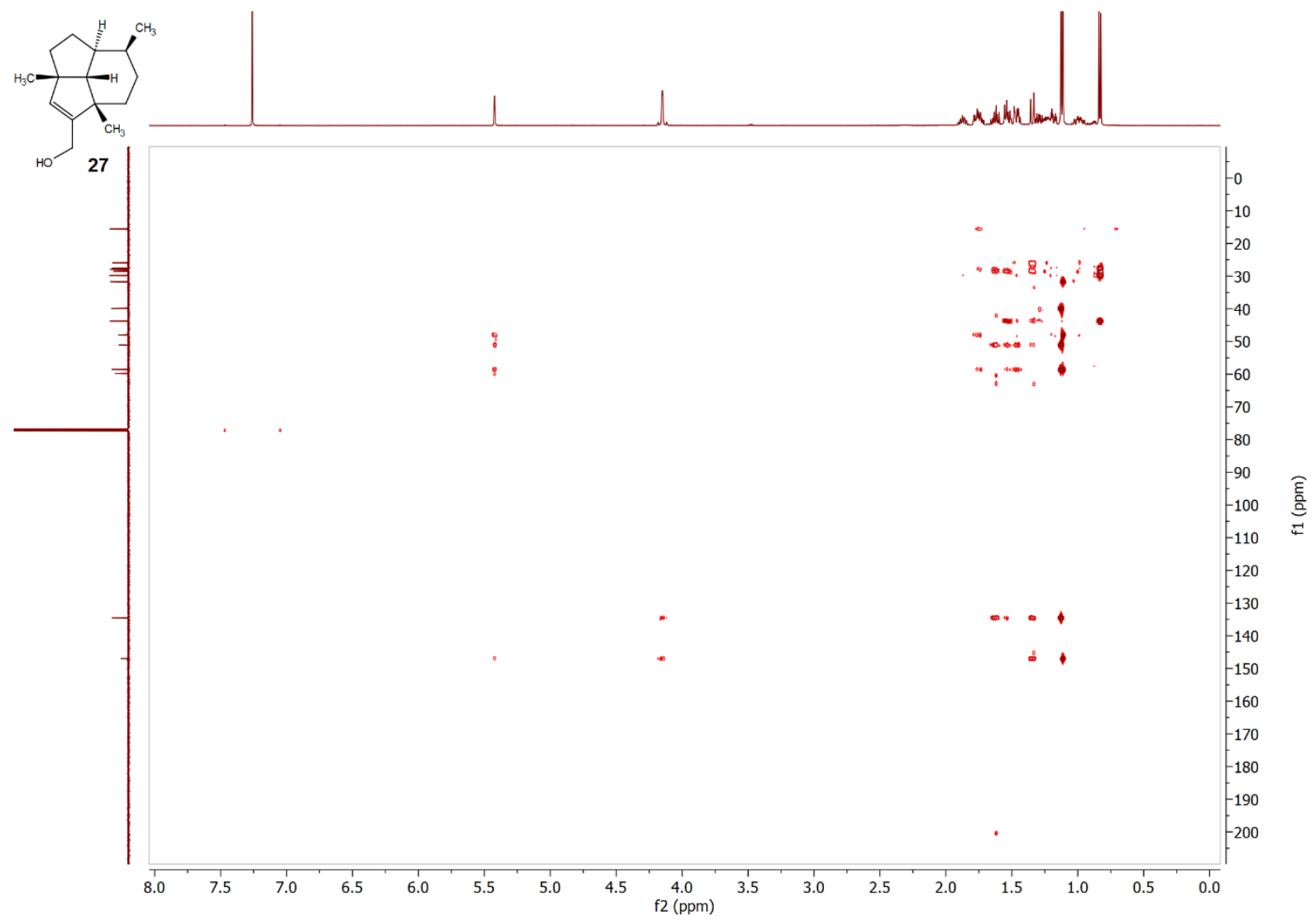




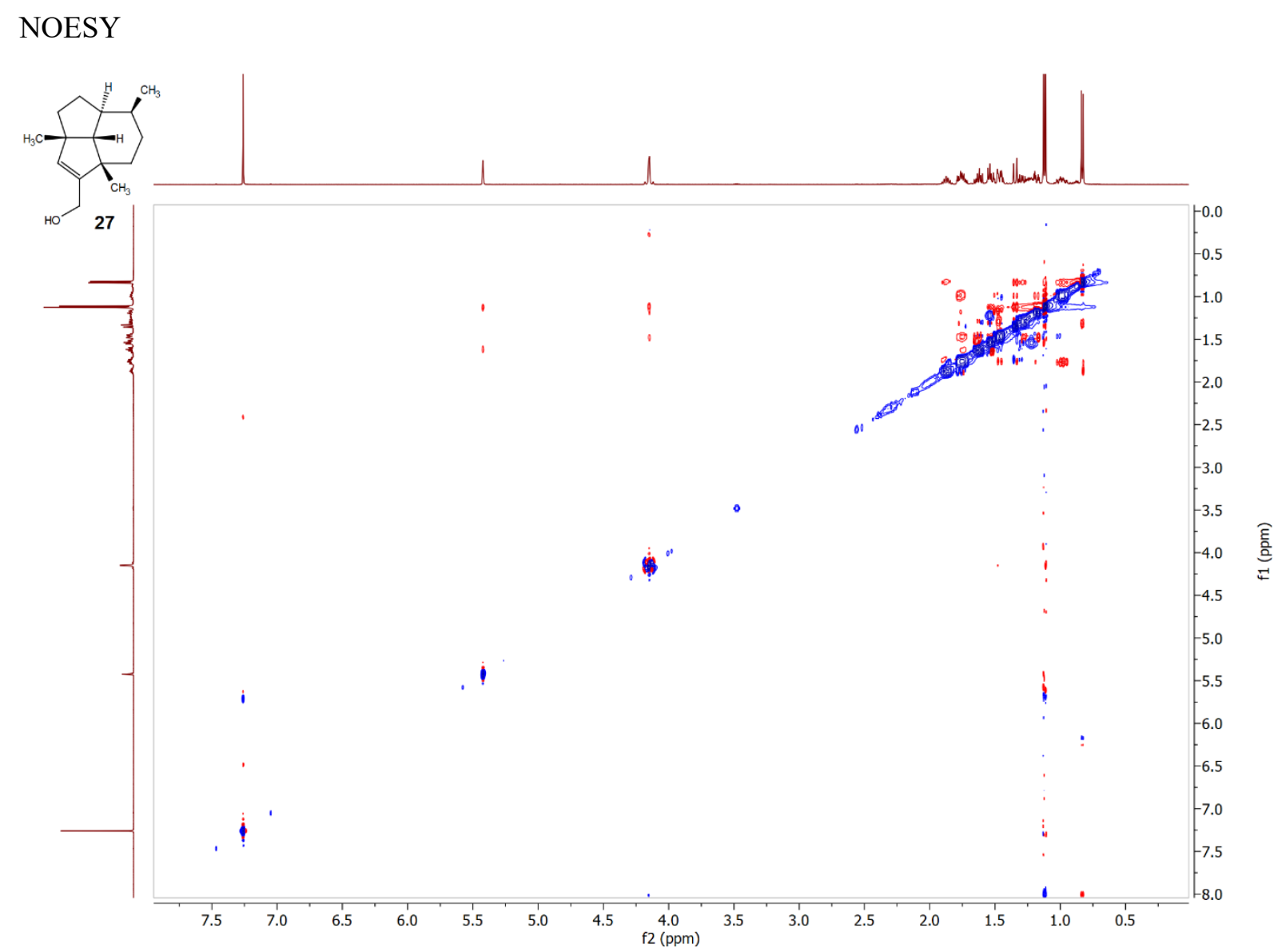


Appendix F: DEPT and 2D-NMR spectra for $\left(2 \mathrm{a} S, 2 \mathrm{a}^{1} R, 4 \mathrm{a} R, 7 S, 7 \mathrm{a} S\right)-2 \mathrm{a}-\mathrm{ethyl}-4,4,7-$ trimethyldecahydro-7aH-cyclopenta[cd]inden-7a-ol [30]

DEPT

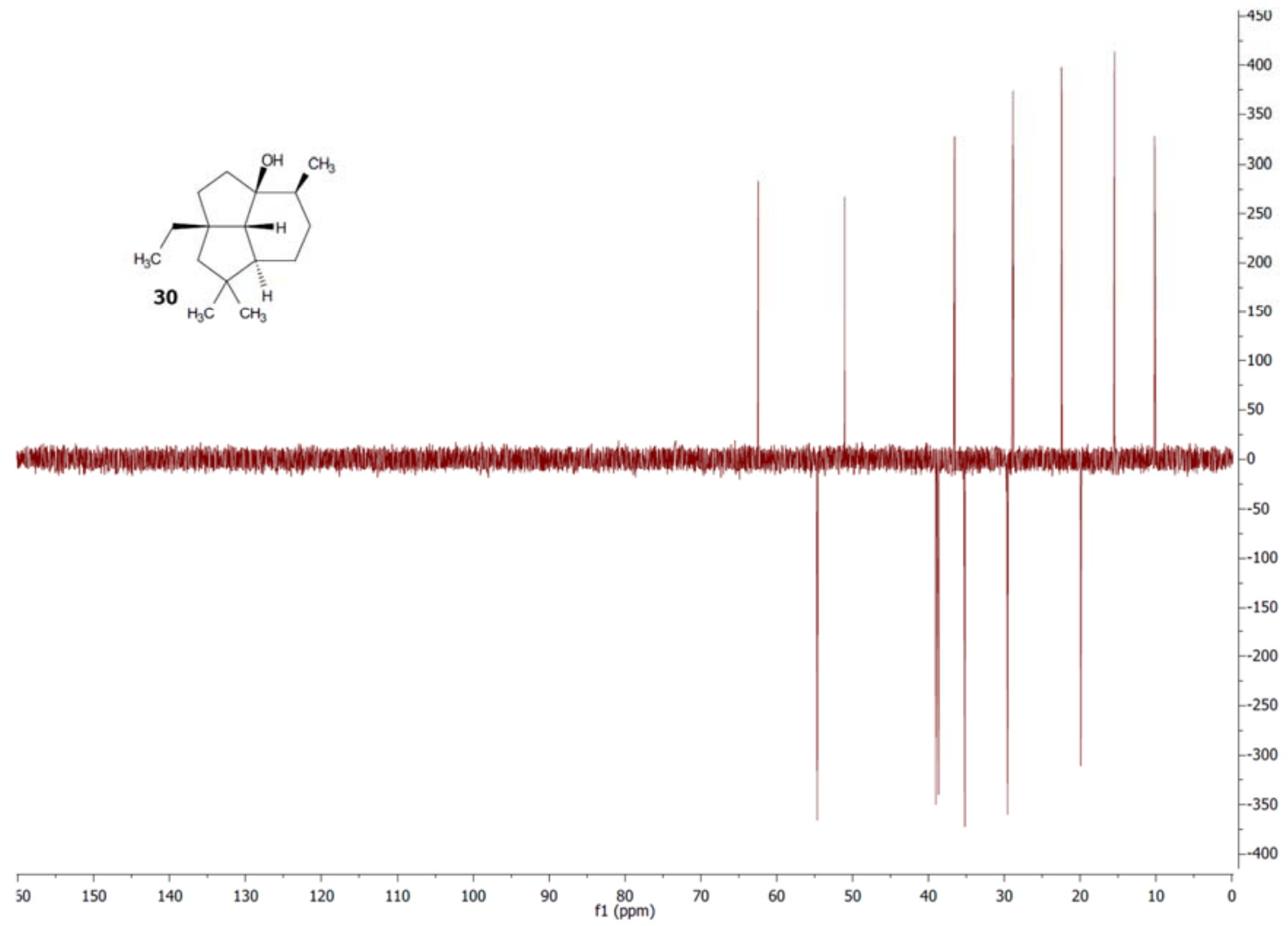

COSY

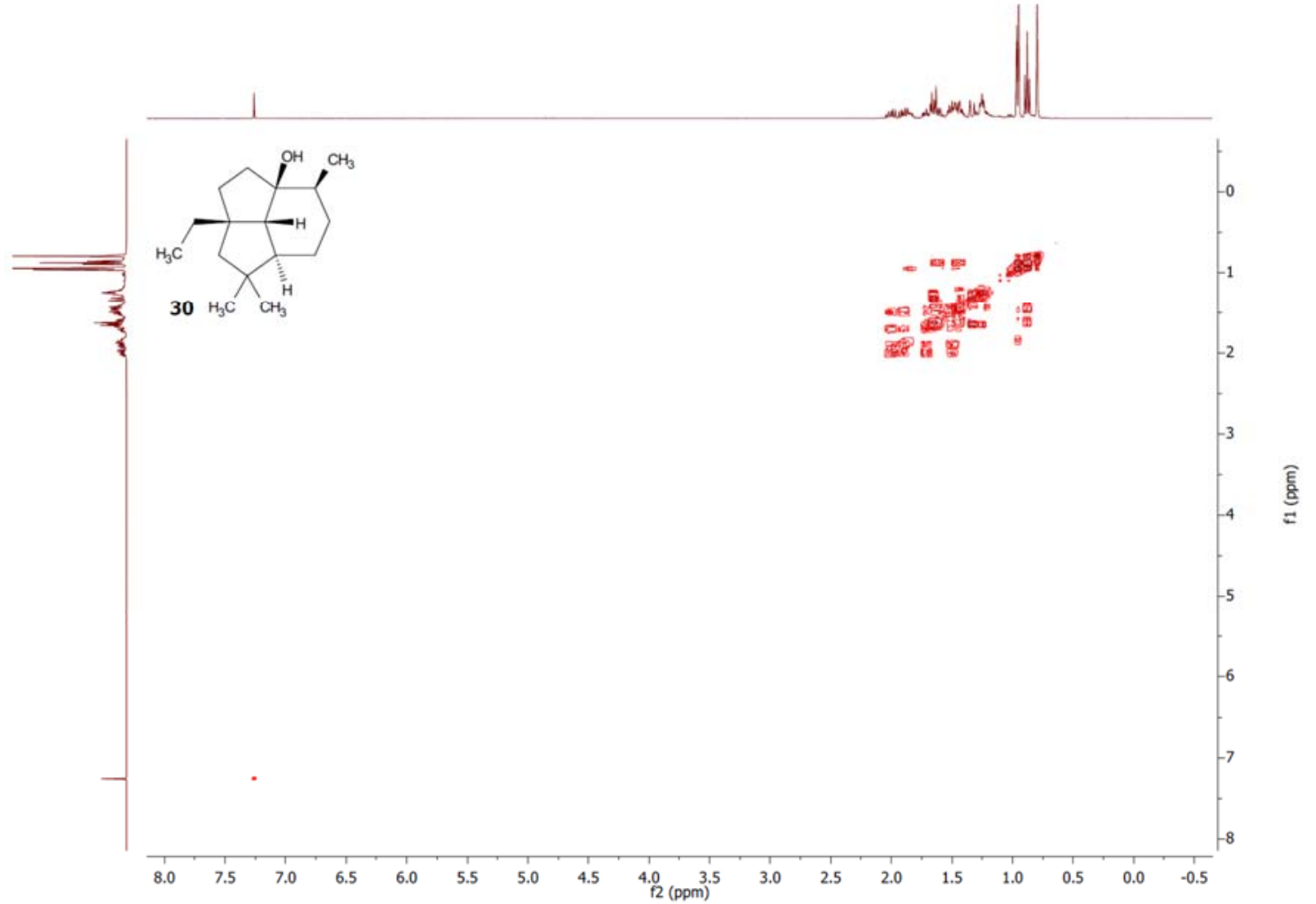


HMQC

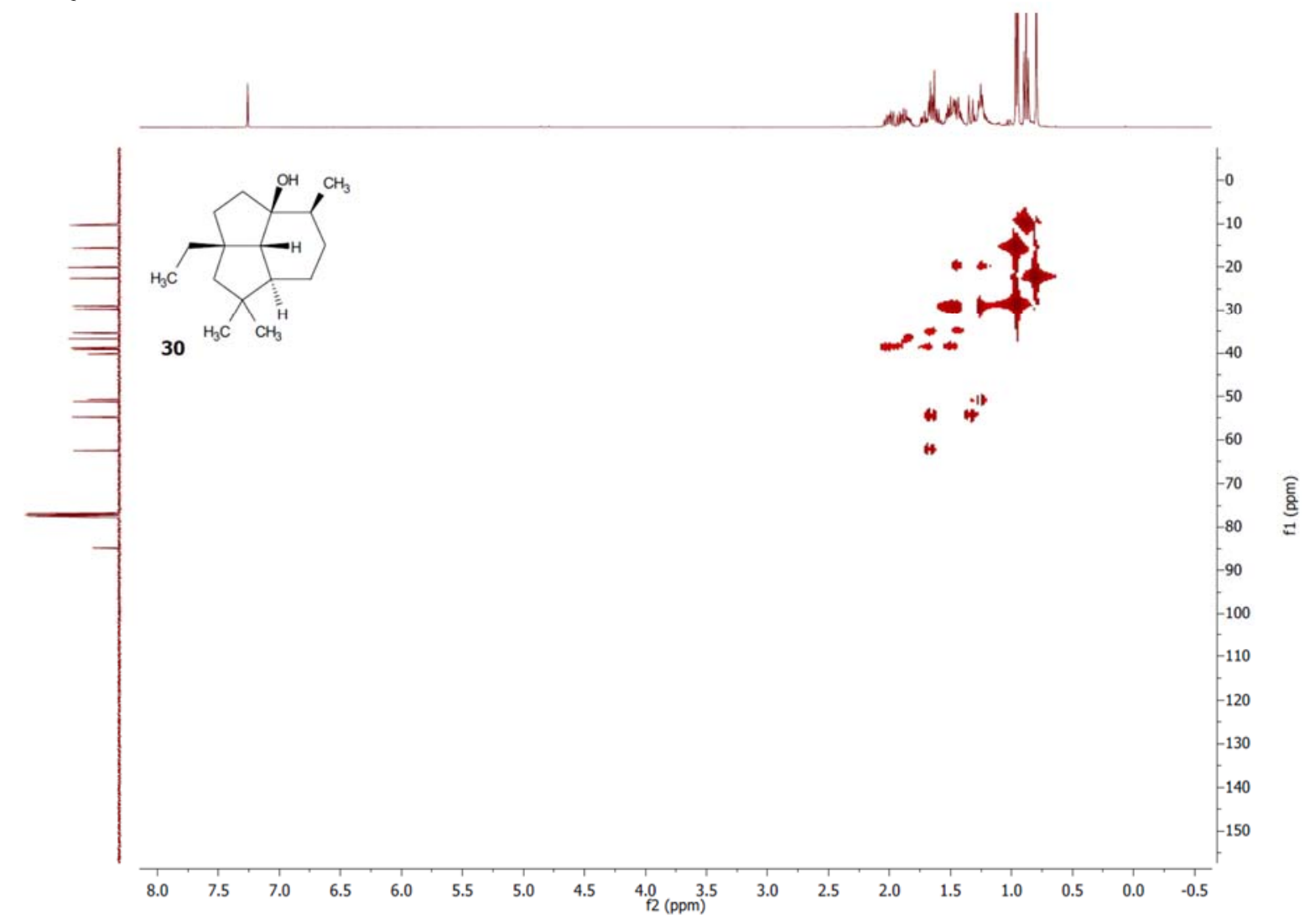

HMBC

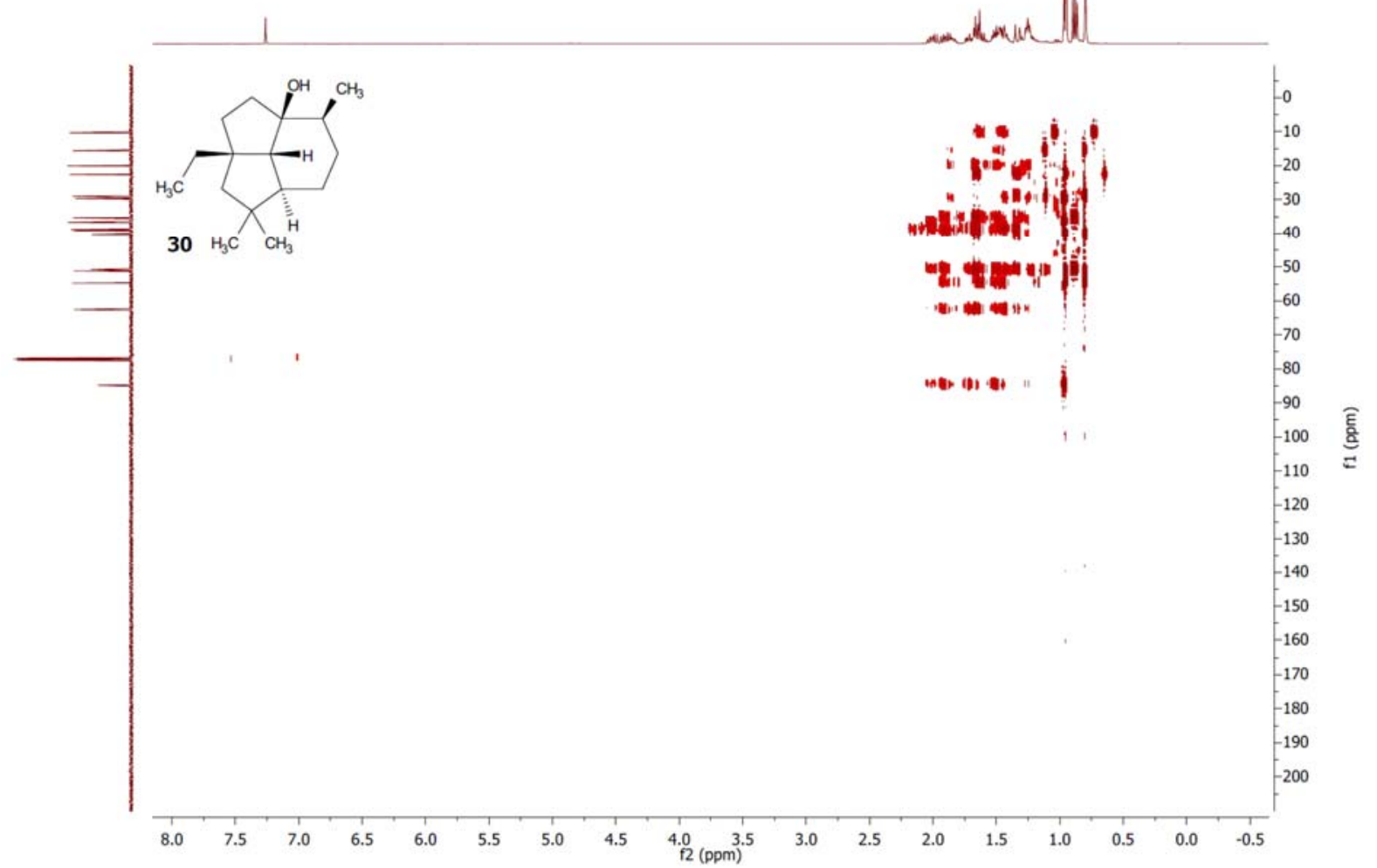


Appendix G: DEPT and 2D-NMR spectra for $\left(2 \mathrm{a} S, 2 \mathrm{a}^{1} \mathrm{R}, \mathbf{4 a}, \mathbf{7}, \mathbf{7}, \mathbf{a} S\right)-2 \mathrm{a}-\mathrm{butyl}-4,4,7-$ trimethyldecahydro-7aH-cyclopenta[cd]inden-7a-ol [32]

DEPT

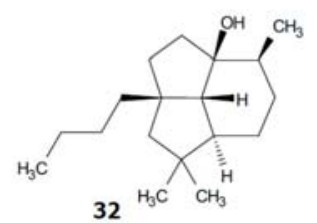

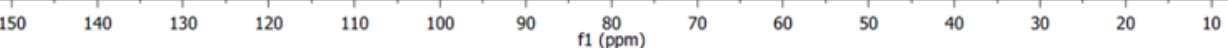

COSY
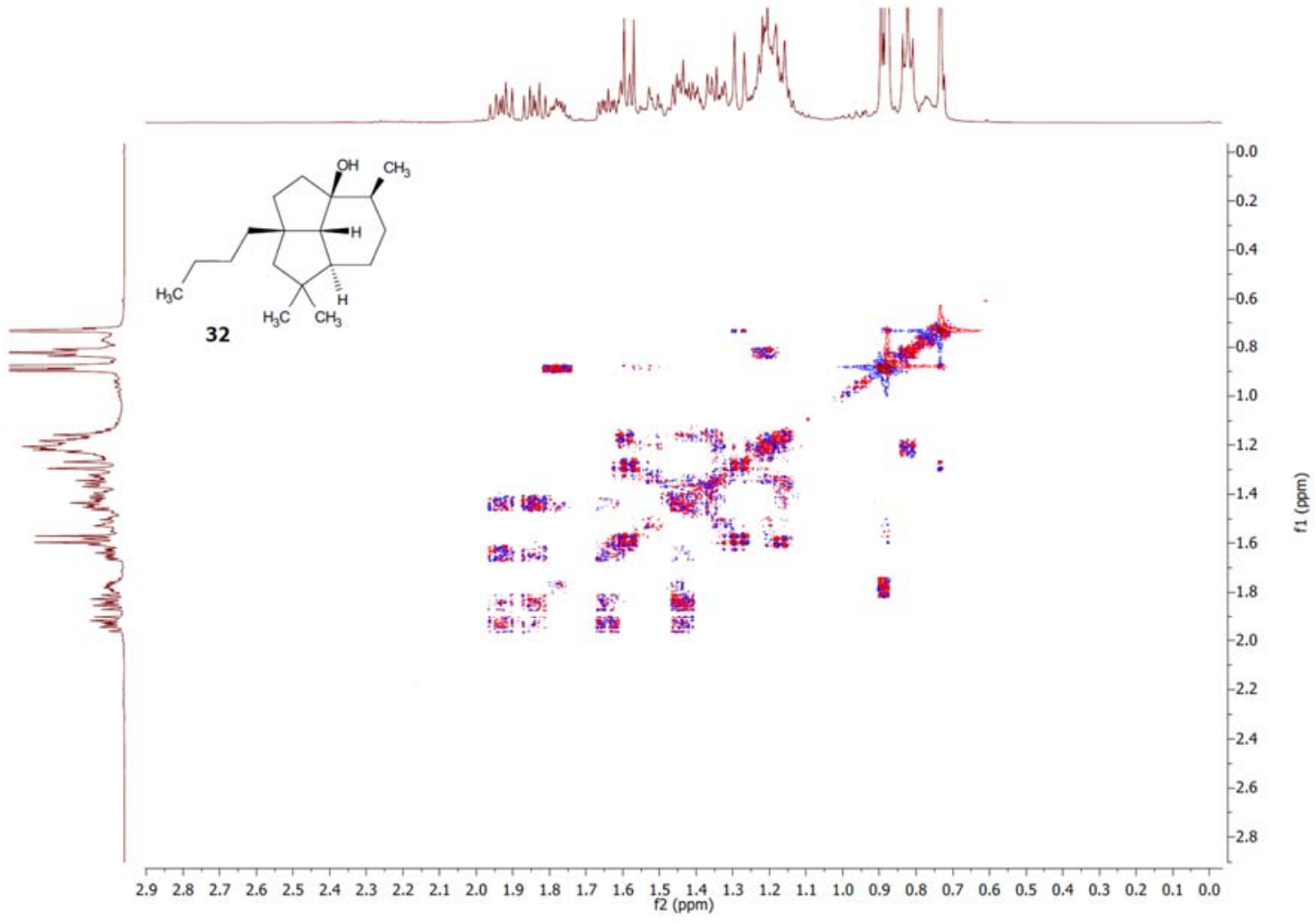


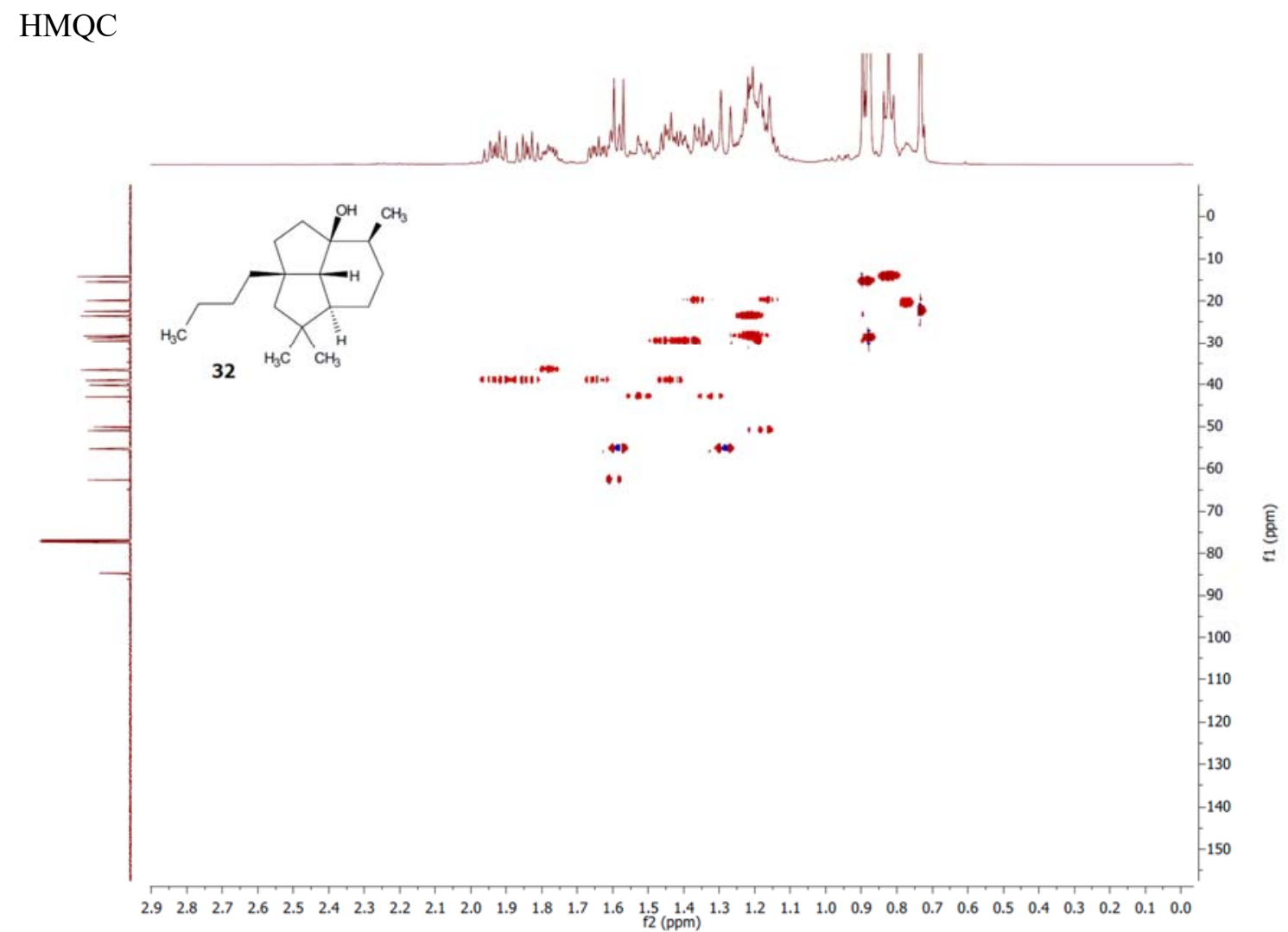

\section{HMBC}

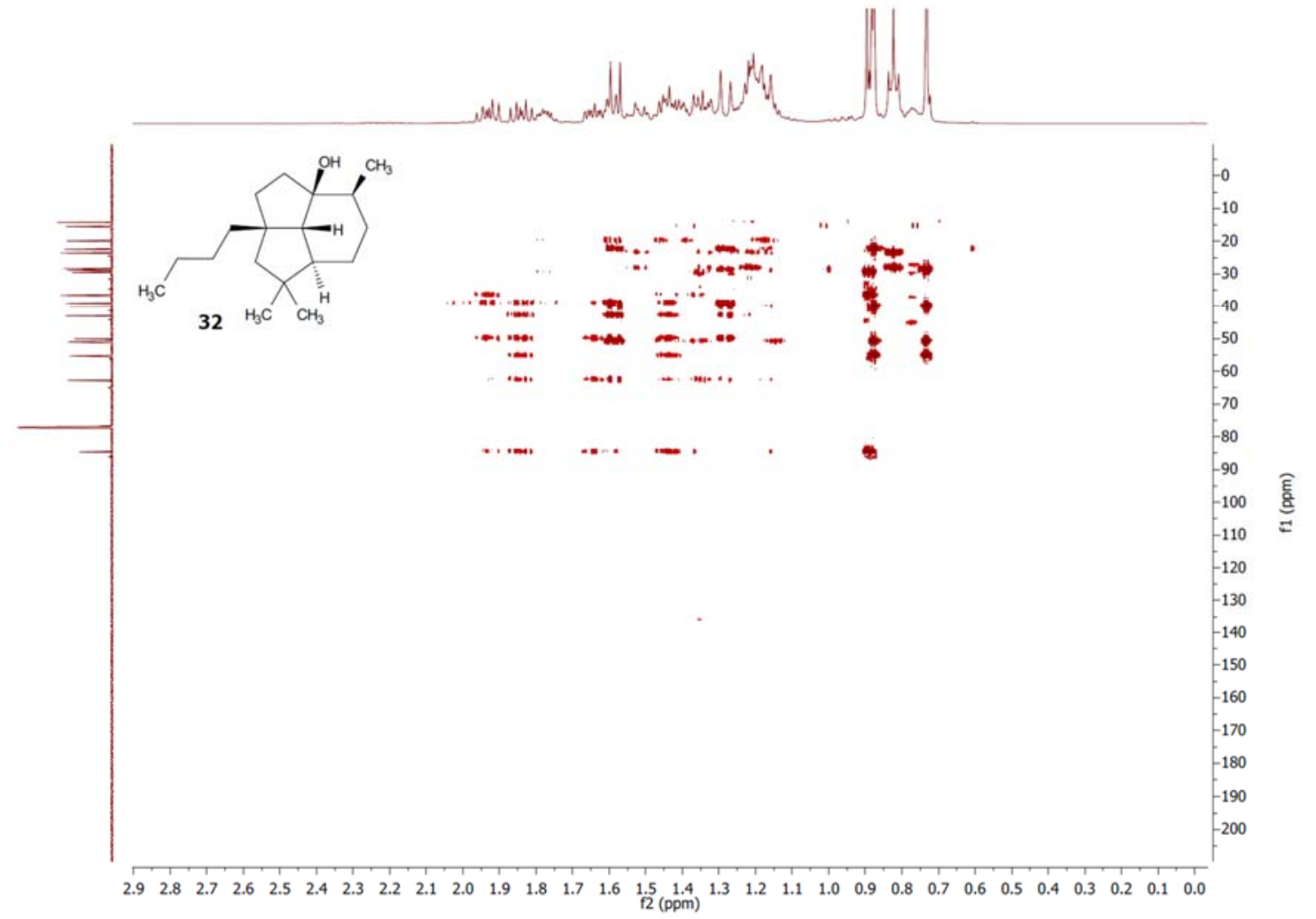


Appendix H: DEPT and 2D-NMR spectra for $\left(2 \mathrm{a} S, 2 \mathrm{a}^{1} \mathrm{R}, 4 \mathrm{a} R, 7 S, 7 \mathrm{a} S\right)-2 \mathrm{a}-$-iso-butyl-4,4,7trimethyldecahydro-7aH-cyclopenta[cd]inden-7a-ol [34]

DEPT

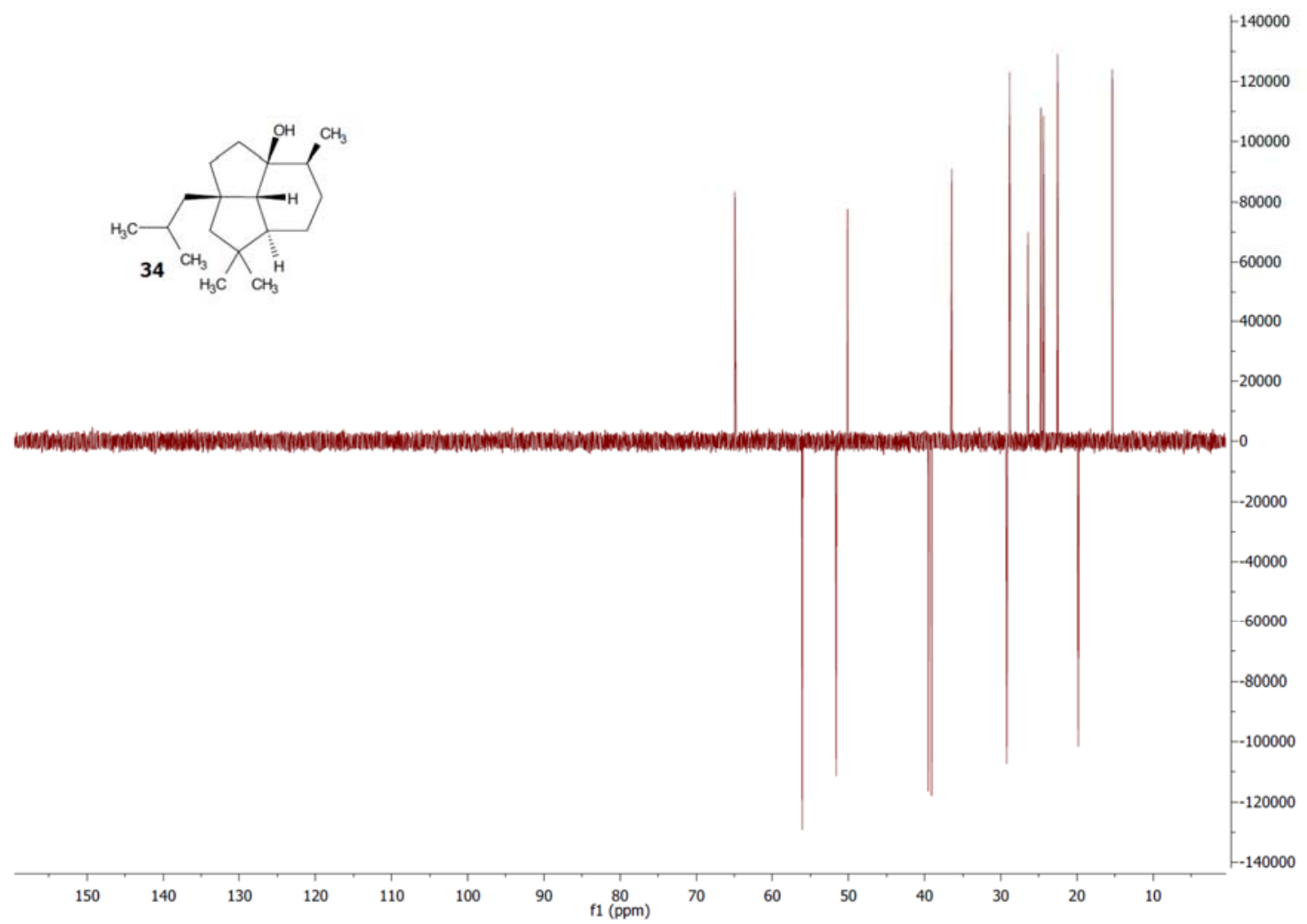

COSY

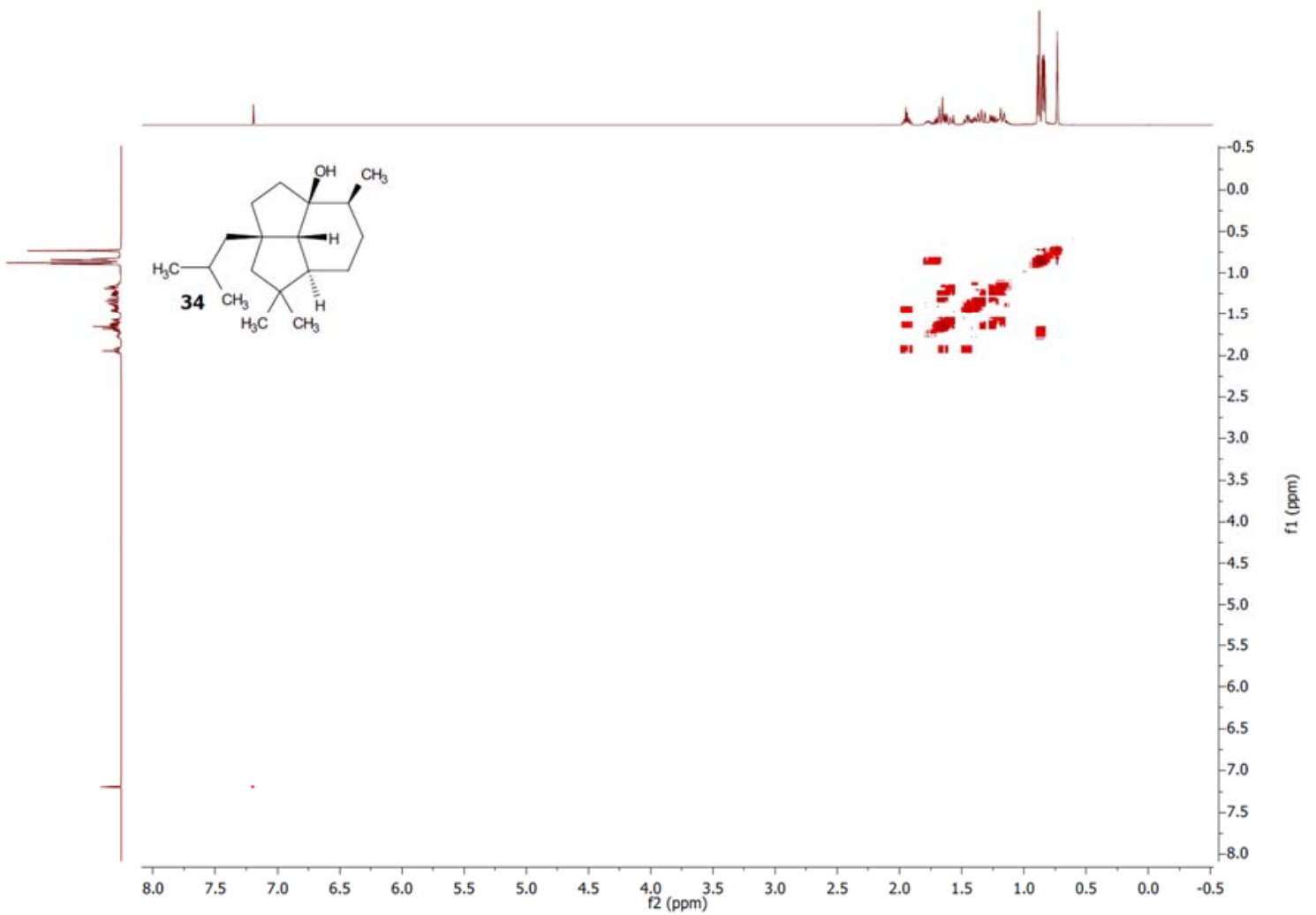




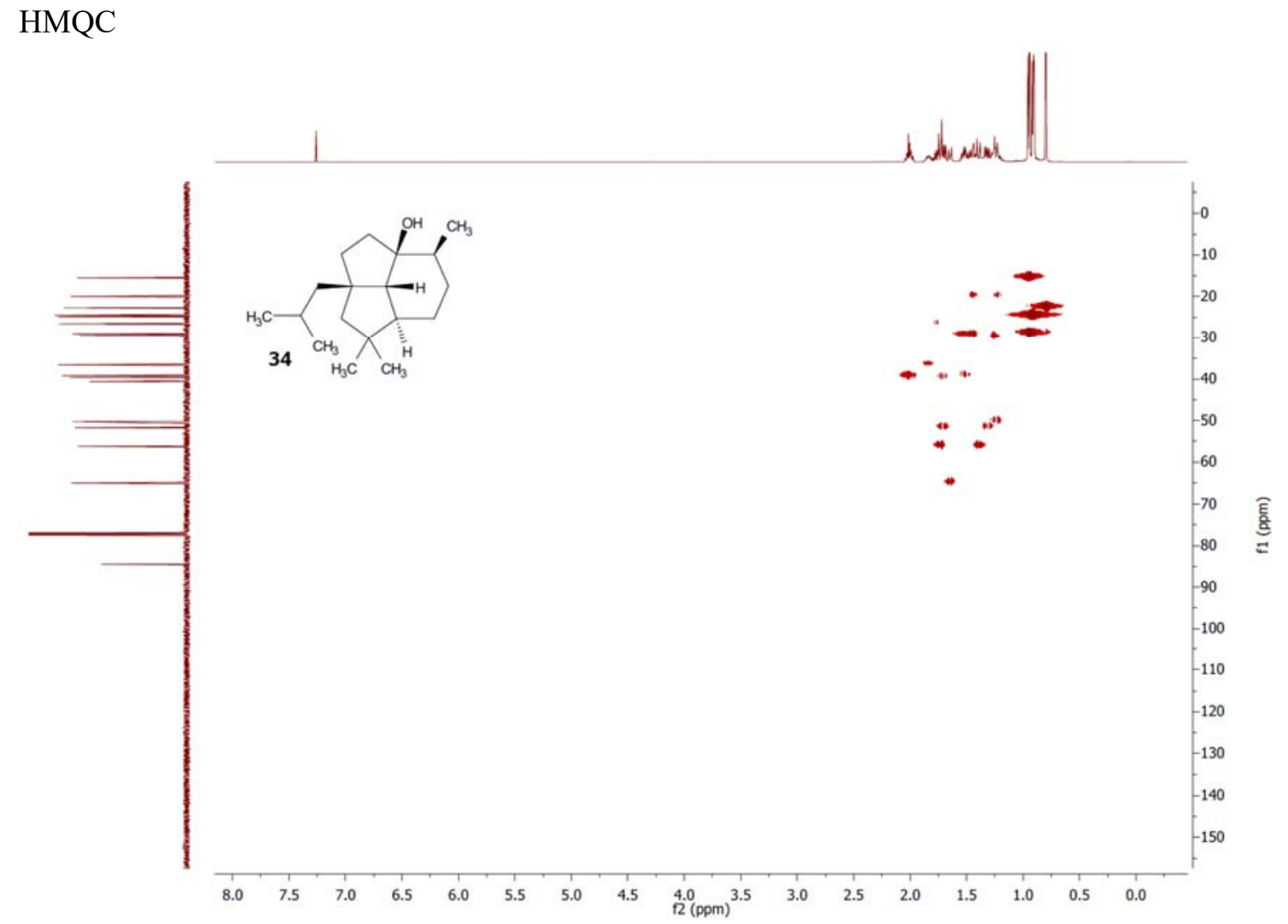

HMBC

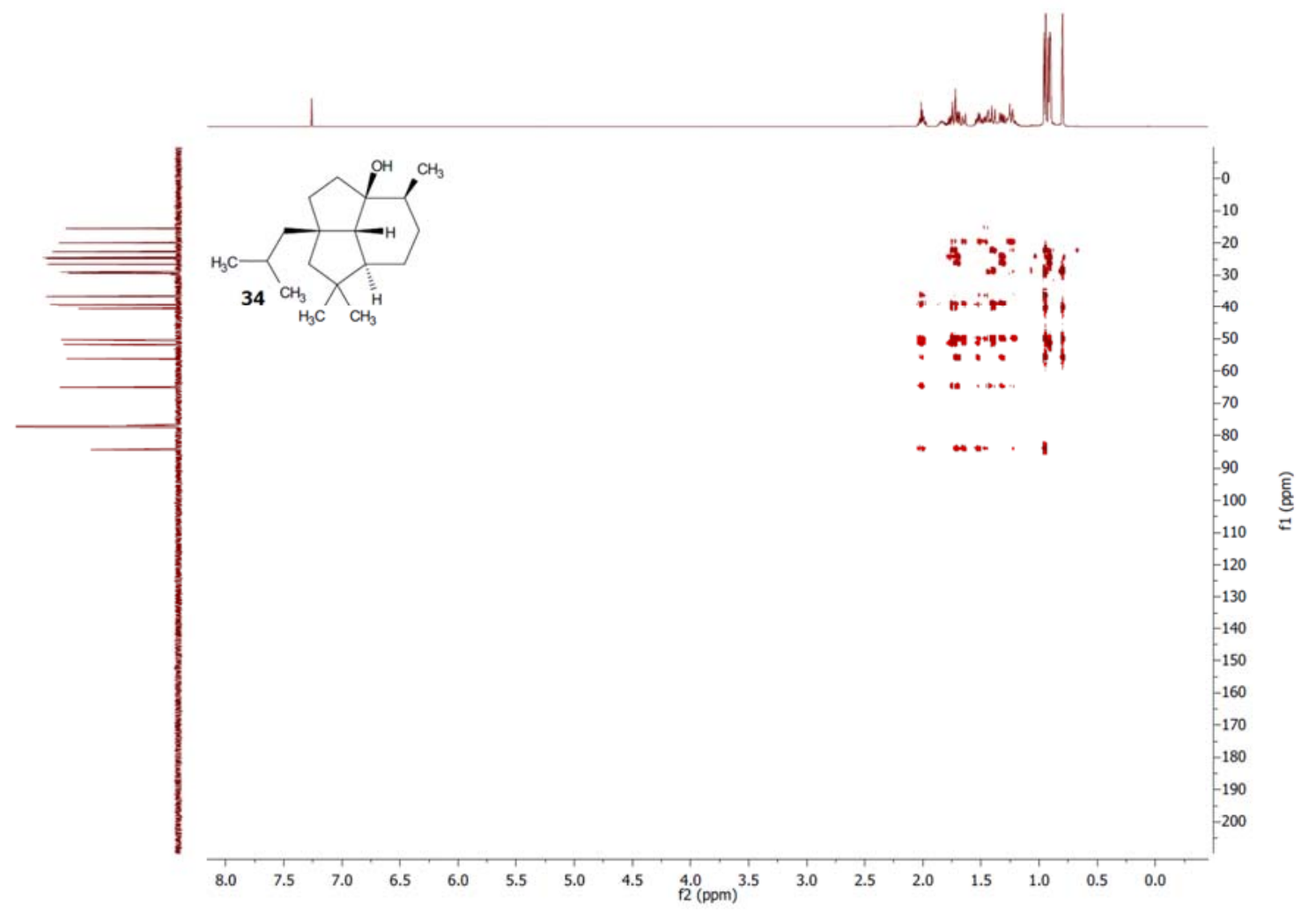


Appendix I: DEPT and 2D-NMR spectra for $\left(2 \mathrm{a} S, 2 \mathrm{a}^{1} R, 4 \mathrm{a} R, 7 S, 7 \mathrm{a} S\right)-2 \mathrm{a}-\mathrm{hexyl}-4,4,7-$ trimethyldecahydro-7aH-cyclopenta[cd]inden-7a-ol [36]

DEPT
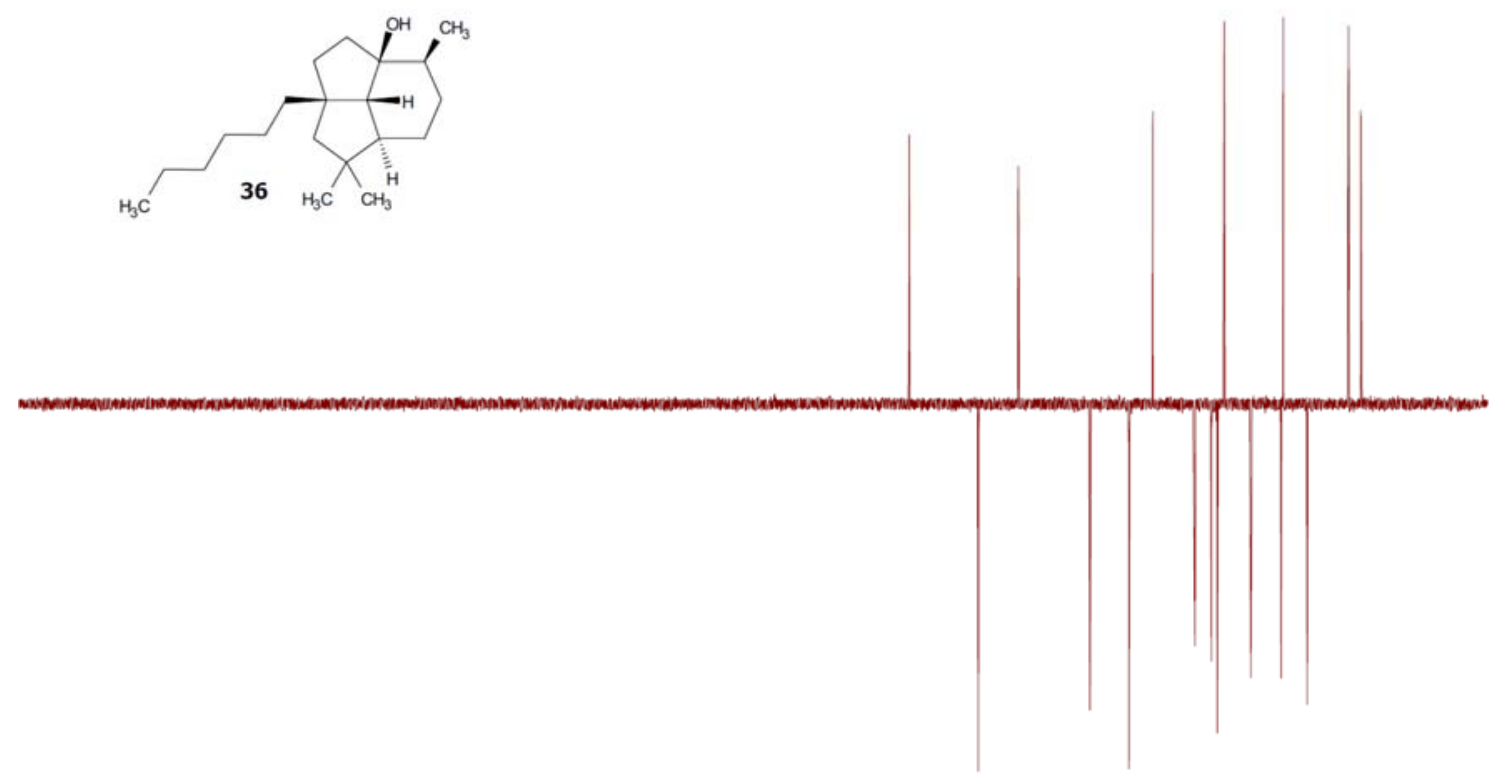

\begin{tabular}{|c|c|c|c|c|c|c|c|c|c|c|c|c|c|}
\hline & 140 & 130 & 120 & 110 & 100 & 90 & 80 & 70 & 60 & 50 & 40 & 30 & 20 \\
\hline
\end{tabular}

$\operatorname{COSY}$

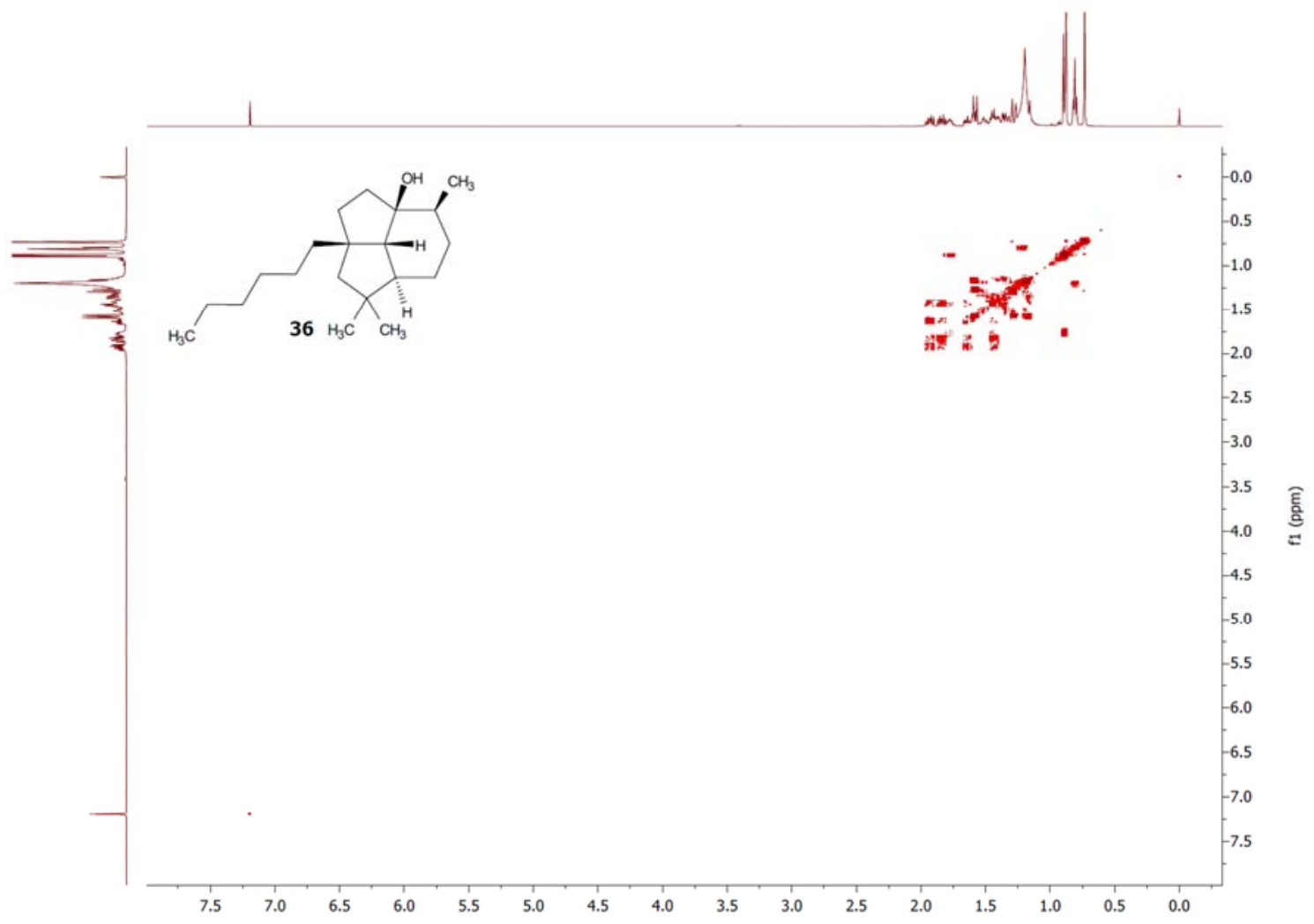




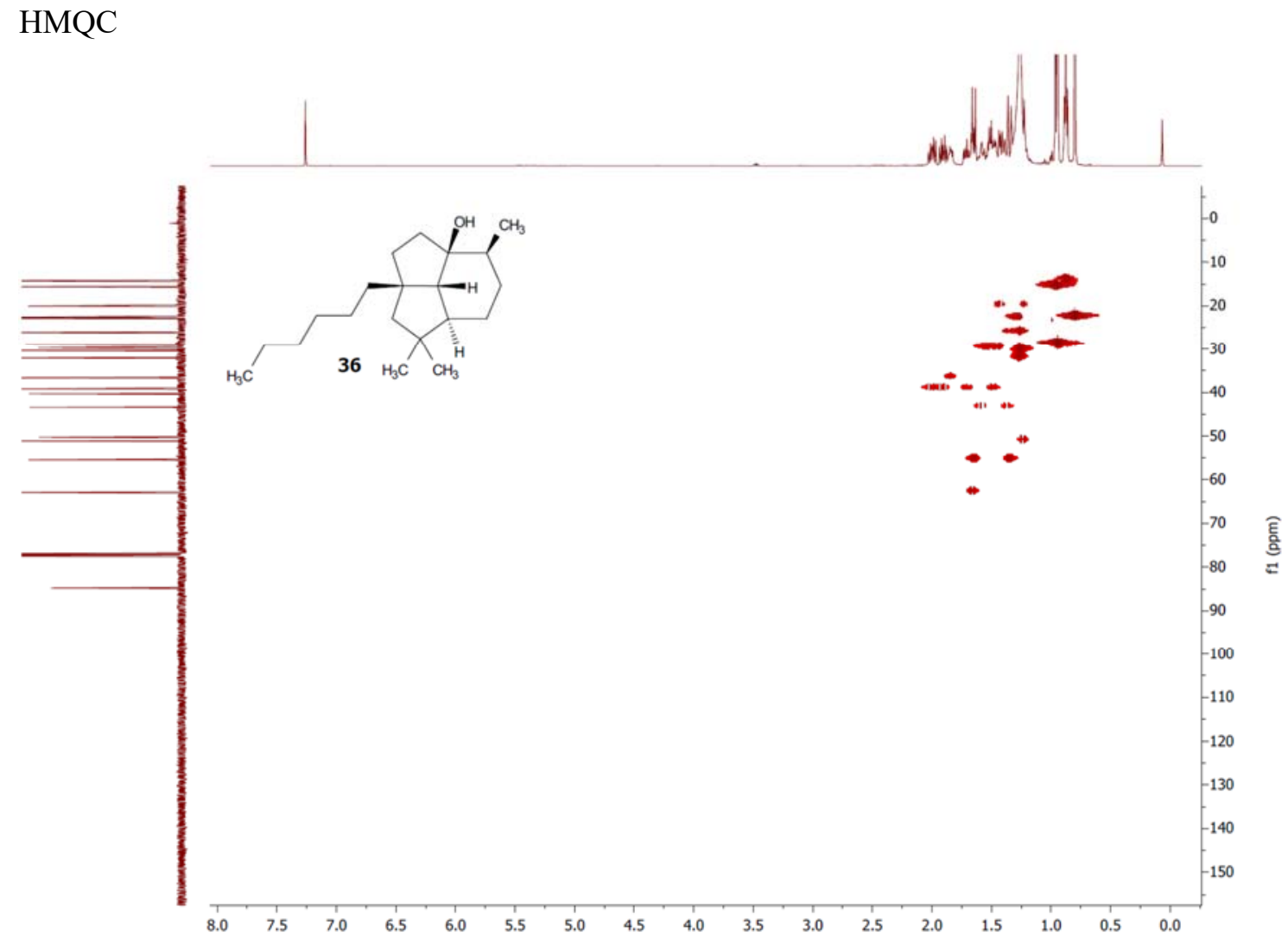

HMBC

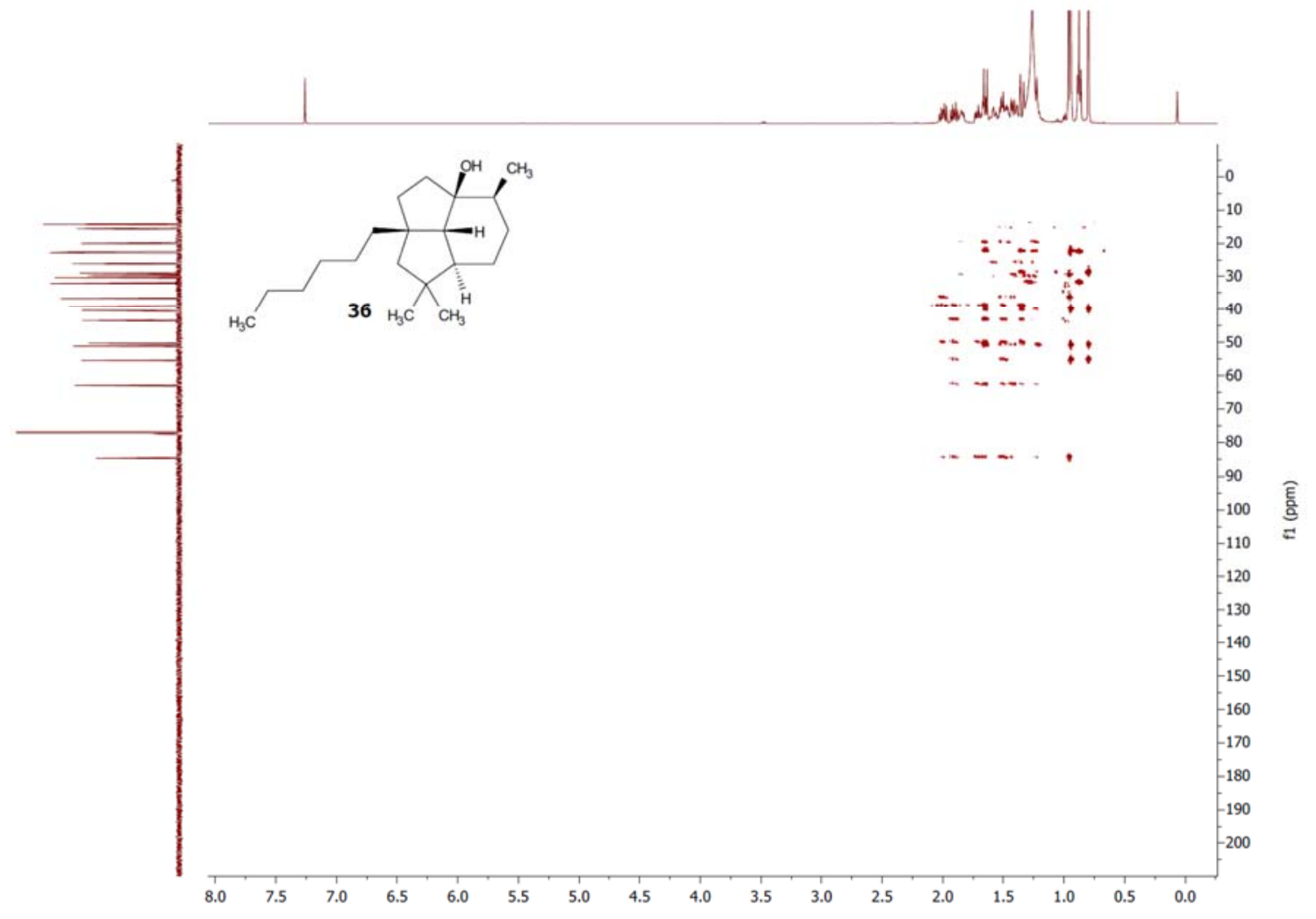


Appendix J: DEPT and 2D-NMR spectra for $\left(2 \mathrm{a} S, 2 \mathrm{a}^{1} \mathrm{R}, 4 \mathrm{a} R, 7 S, 7 \mathrm{a} S\right)-2 \mathrm{a}-\mathrm{octyl}-4,4,7-$ trimethyldecahydro-7aH-cyclopenta[cd]inden-7a-ol [38]

DEPT

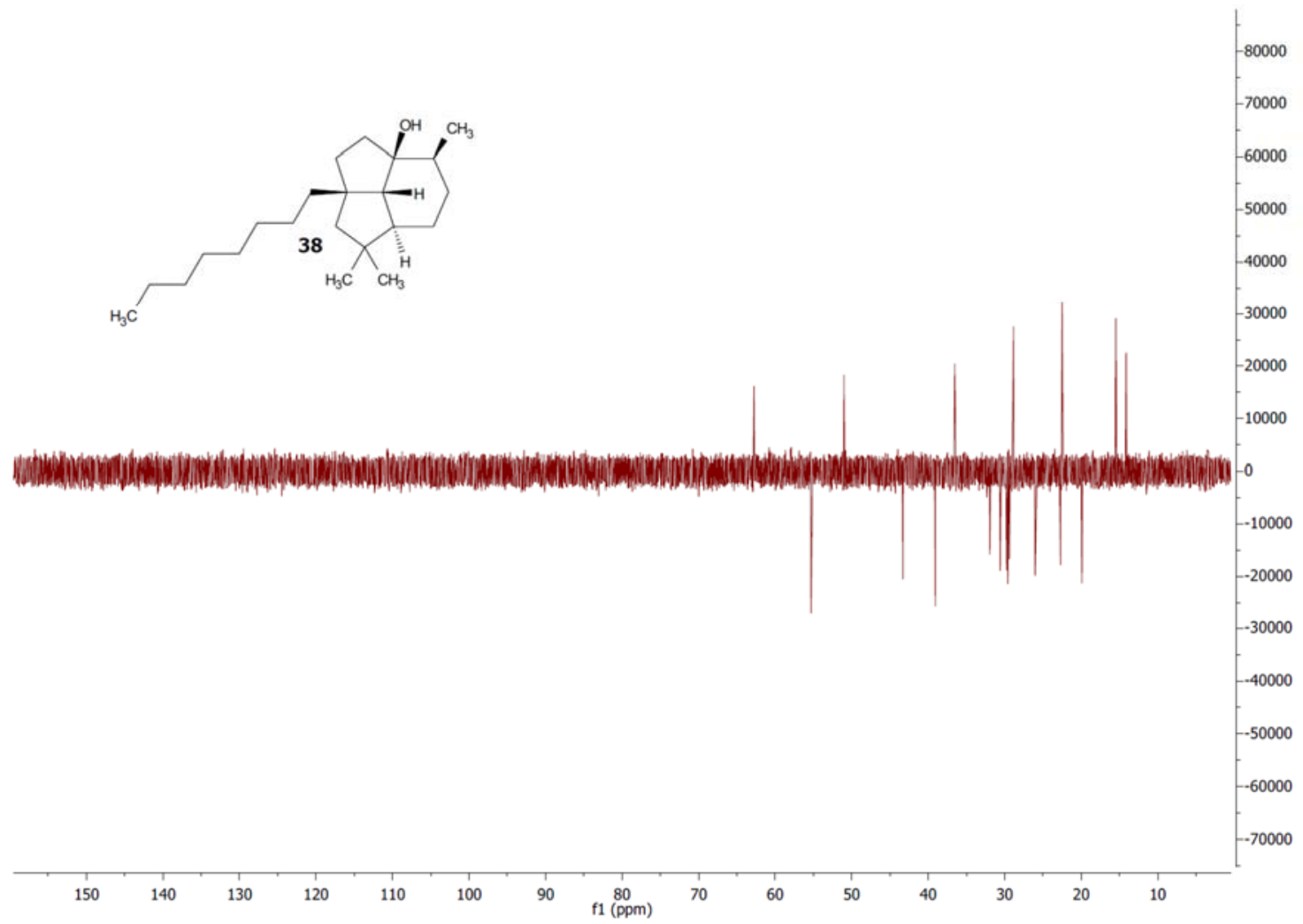

COSY

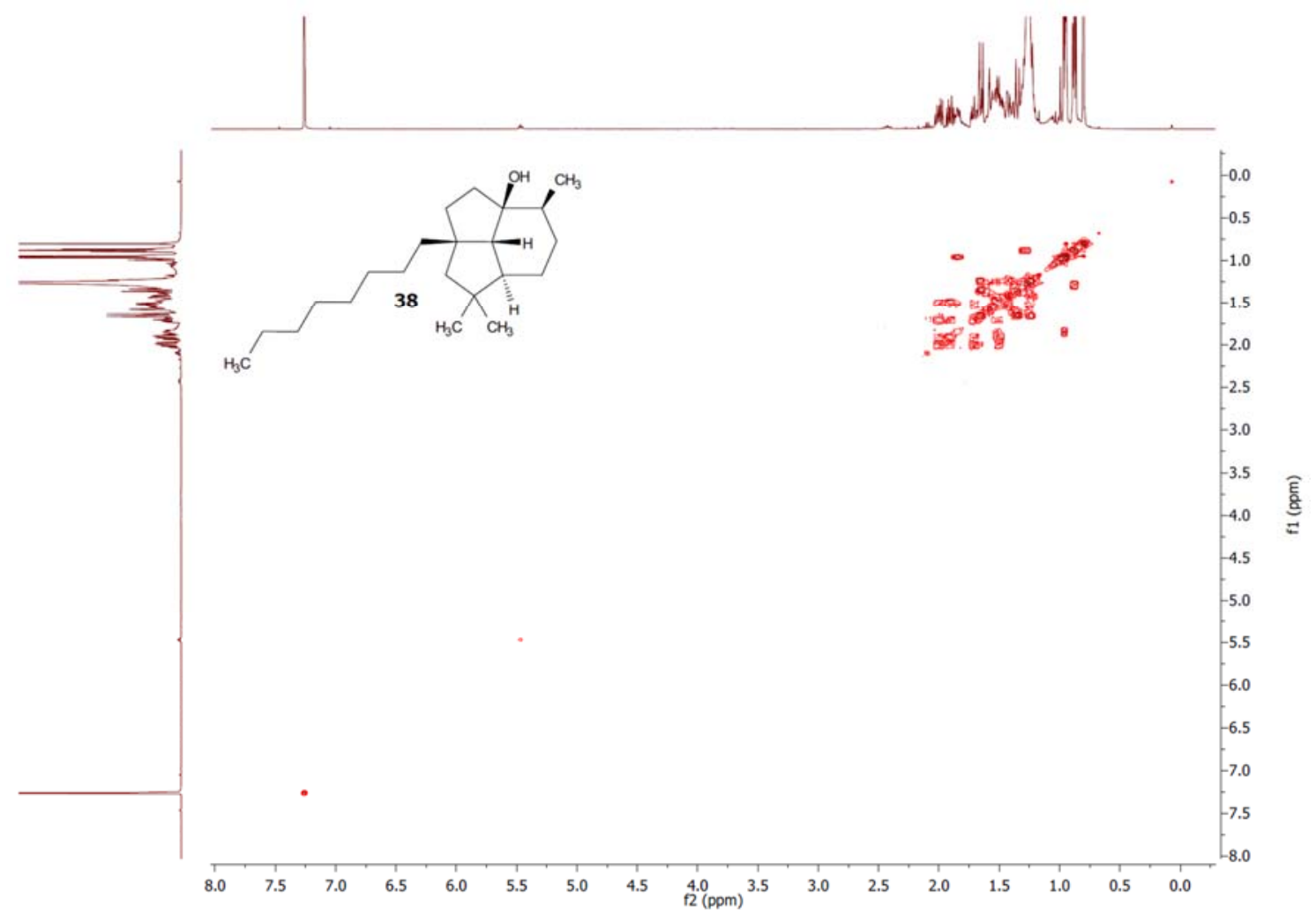




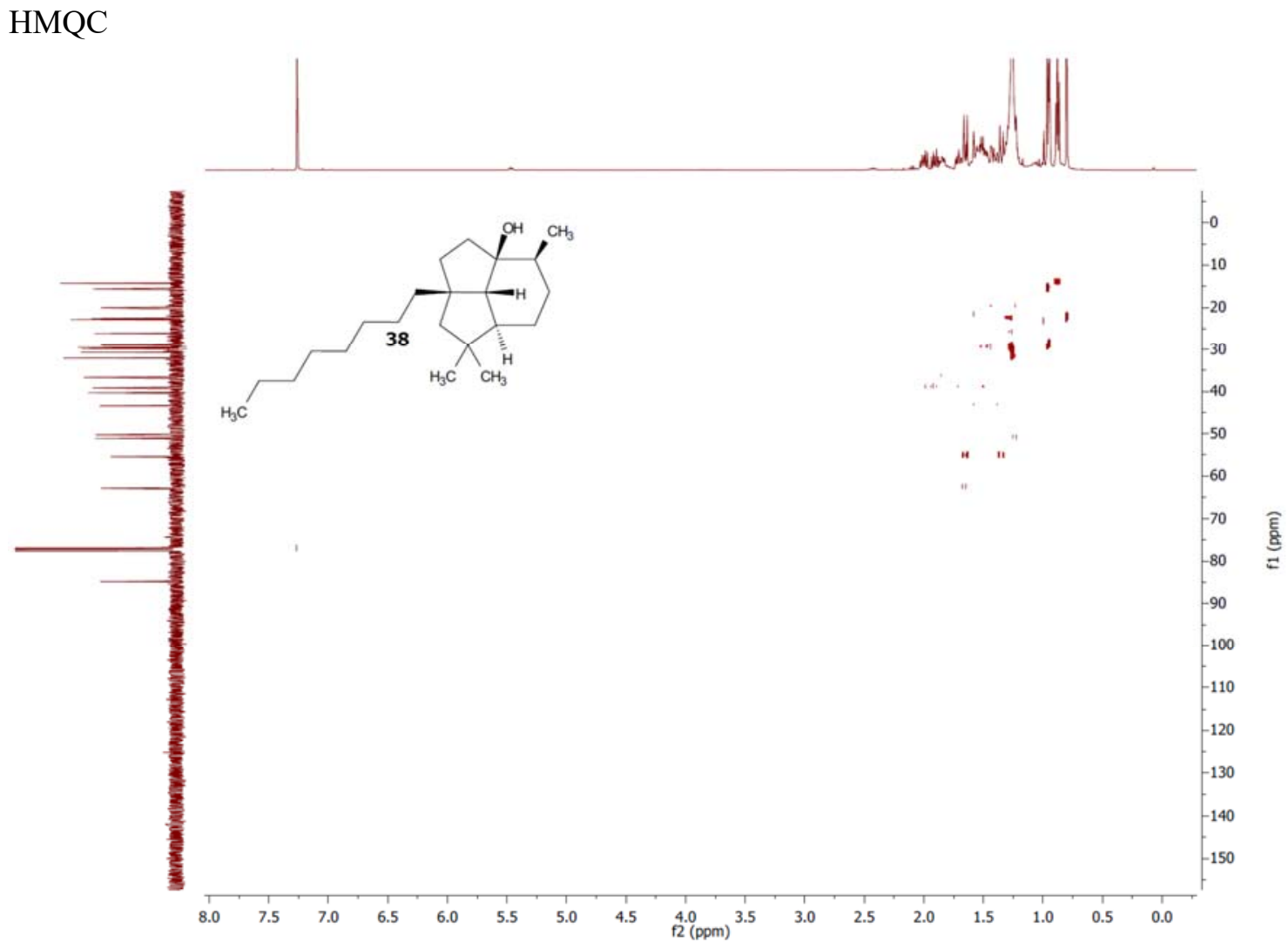

HMBC

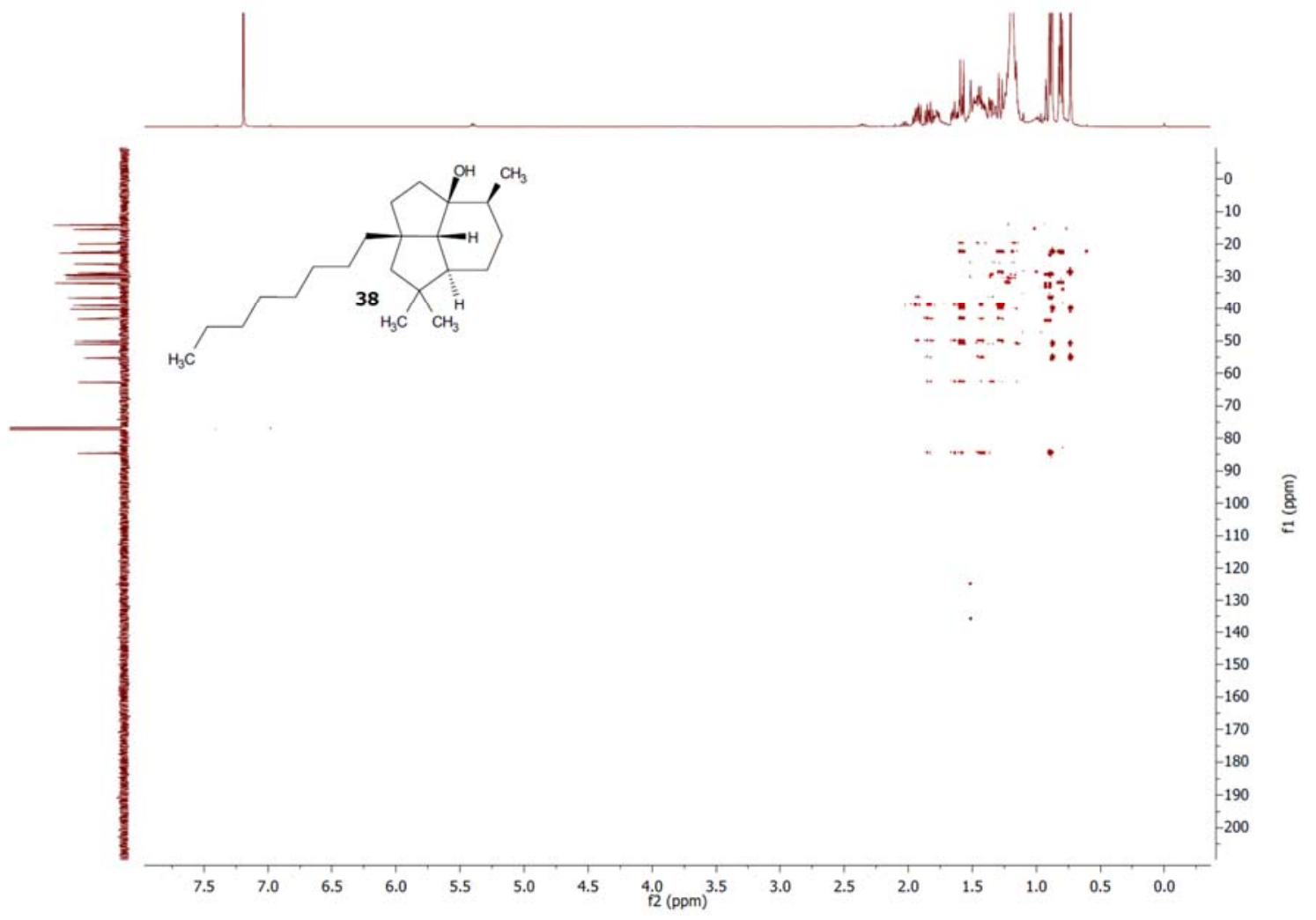

